\title{
Numerical Flight Testing of a Tube-launched Transformable Micro Air Vehicle
}

\author{
William L. Vogel \\ West Virginia University
}

Follow this and additional works at: https://researchrepository.wvu.edu/etd

\section{Recommended Citation}

Vogel, William L., "Numerical Flight Testing of a Tube-launched Transformable Micro Air Vehicle" (2012). Graduate Theses, Dissertations, and Problem Reports. 4932.

https://researchrepository.wvu.edu/etd/4932

This Thesis is protected by copyright and/or related rights. It has been brought to you by the The Research Repository @ WVU with permission from the rights-holder(s). You are free to use this Thesis in any way that is permitted by the copyright and related rights legislation that applies to your use. For other uses you must obtain permission from the rights-holder(s) directly, unless additional rights are indicated by a Creative Commons license in the record and/ or on the work itself. This Thesis has been accepted for inclusion in WVU Graduate Theses, Dissertations, and Problem Reports collection by an authorized administrator of The Research Repository @ WVU. For more information, please contact researchrepository@mail.wvu.edu. 


\title{
Numerical Flight Testing of a Tube-launched Transformable Micro Air Vehicle
}

\author{
William L. Vogel \\ Thesis submitted to the \\ College of Engineering and Mineral Resources \\ at West Virginia University \\ in partial fulfillment of the requirements for the degree of \\ Masters of Science \\ In \\ Aerospace Engineering \\ Wade W. Huebsch, Ph.D., Chair \\ Patrick H. Browning, Ph.D. \\ Gary J. Morris, Ph.D. \\ Department of Mechanical and Aerospace Engineering \\ Morgantown, West Virginia \\ 2012
}

Keywords: Computational Fluid Dynamics, 6-DOF, Fluent, ICEM CFD, Dynamic Mesh, k- $\omega$ SST Model, Low Reynolds Number Flight, Micro Aerial Vehicle, Unmanned Aerial Vehicle 


\begin{abstract}
Numerical Flight Testing of a Tube-launched Transformable Micro Air Vehicle
\end{abstract}

\author{
William L. Vogel
}

The micro air vehicle (MAV) is a relatively new class of Unmanned Aerial Vehicles (UAVs) and has drawn much interest in recent years within the aerospace industry, especially for military surveillance applications. The aerodynamic characteristics of existing MAV designs have been documented in aeronautics literature; however, the MAV presented in this research had unusual design specifications in which the overall mass and cruise speed were greater in comparison to the existing designs. Additionally, the MAV was designed to transform from ballistic flight to aircraft flight for the purpose of gaining performance improvements in both range and accuracy. This new MAV design consists of a two-stage deployment process of its control surfaces in which the tail fins are first deployed immediately after being launched from a tube system followed by deployment of the wings at the apex of the original ballistic trajectory. This research effort incorporated a relatively new simulation tool that couples computational fluid dynamics (CFD) analysis with 6-DOF flight prediction. This coupling is being called numerical flight testing; it has allowed the numerical prediction for the aerodynamic flight behavior of the MAV in free flight motion. Prior to conducting numerical simulations of free flight motion, several, more traditional, CFD simulations were executed for a range of angles of attack in which the airflow traveled relative to the fixed body. Once the aerodynamic results were obtained, the MAV was optimized for the maximum lift-to-drag and the tail was trimmed to balance the forces on the body and generate negligible rotation about the pitch axis. With the known location of the center of gravity, the baseline was determined to be gyroscopically stable. This was confirmed with experimental data. A major concern addressed in this research was the resulting dynamics of the MAV once deployment of the tail and wings was complete, and how this affected the follow-on flight dynamics. Therefore, a total of four numerical flight tests were conducted in the MAV's various configurations. Because of the limited computational performance capabilities, an analysis of the MAV's complete flight trajectory could not be achieved. The first simulation was executed on the MAV in the stowable configuration (i.e. baseline) immediately upon launch from the barrel of the tube to evaluate its dynamic stability. Upon deployment of the tail, a simulation was performed to provide a prediction of the projectile's stability as well as its rate of spin decay and time required to de-spin. The final two simulations were conducted upon deployment of the wings using two different tail configurations in which its longitudinal and lateral-directional stability were analyzed. In addition to this analysis, the aerodynamic force characteristics were examined. 


\section{Acknowledgements}

This research project would not have been possible without the support of many people. I would like to express thanks to my mother and father for giving me the confidence and encouragement to continue my education and to pursue my desire to work in the aerospace industry.

I would like to express my appreciation to Tom Scarberry of Chemring Ordnance for providing me with experimental verification that supported my research.

I would like to give thanks to former masters student Shanti Hamburg for his advice and support throughout my entire graduate studies. Additionally, I would like to thank Dr. Jay Wilhelm for solving issues related to executing my CFD simulations on the cluster machines.

Many thanks to my committee, Dr. Patrick Browning, Dr. Gary Morris, and Dr. Wade Huebsch for their support in being available to answer questions as well as providing suggestions and edits to my thesis. Special thanks to my advisor, Dr. Huebsch, for providing me with additional computer resources to advance my CFD research studies.

Sincerely,

William L. Vogel 


\section{Table of Contents}

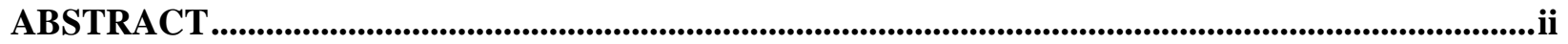

Acknowledgements ..................................................................................................................................................................

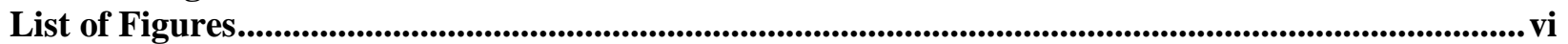

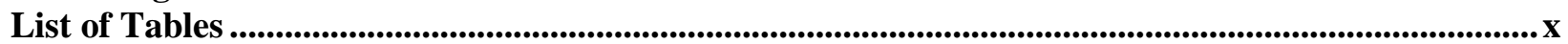

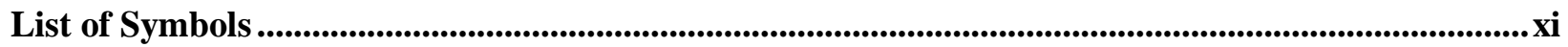

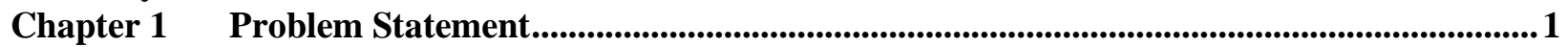

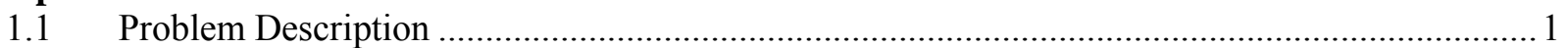

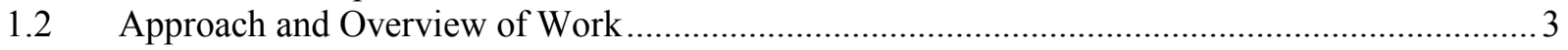

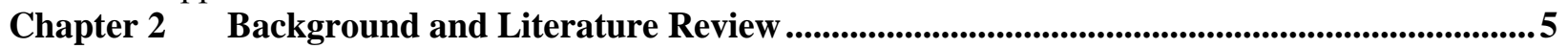

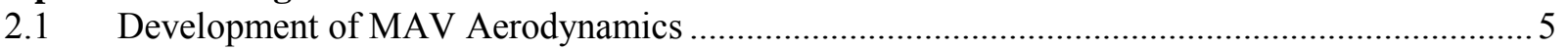

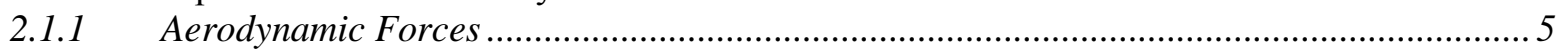

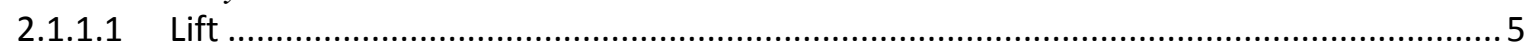

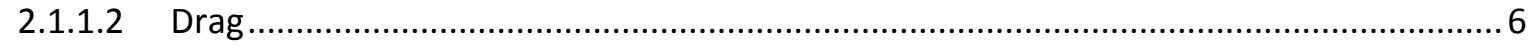

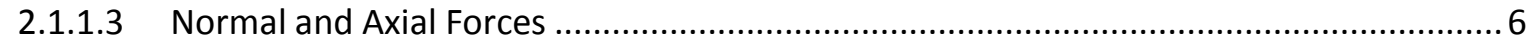

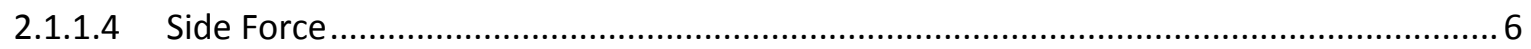

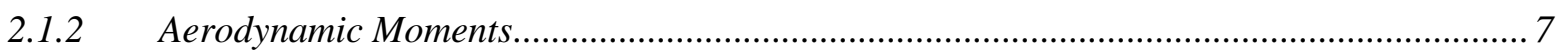

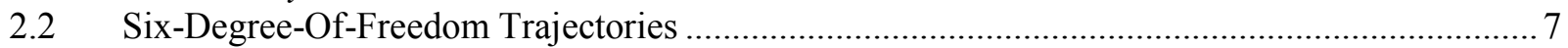

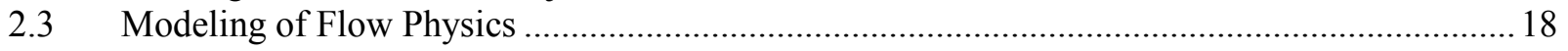

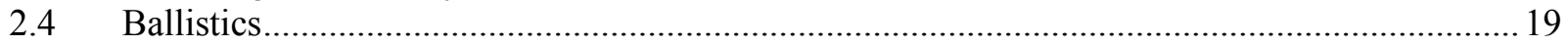

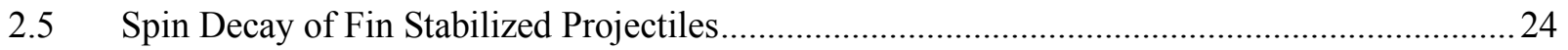

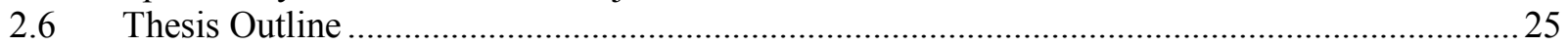

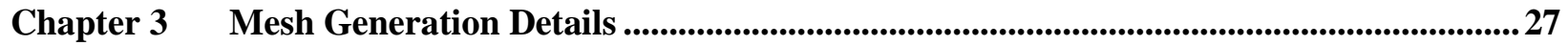

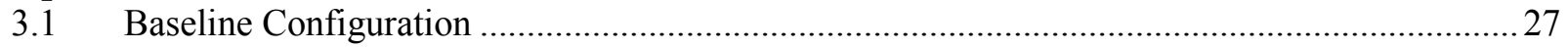

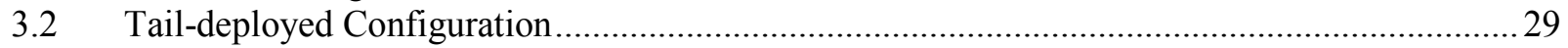

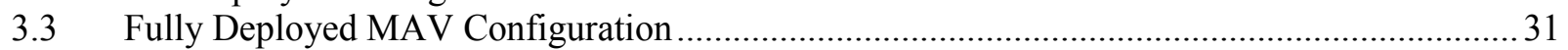

Chapter 4 Fixed Body CFD Analysis..........................................................................................................34

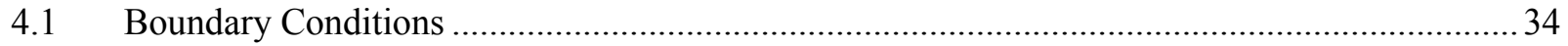

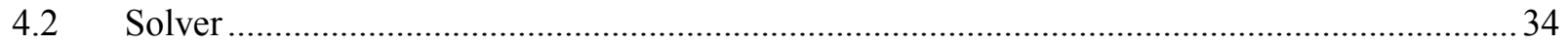

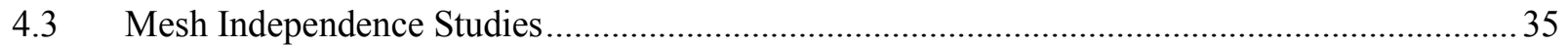

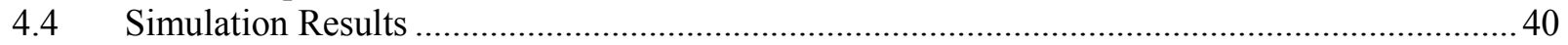

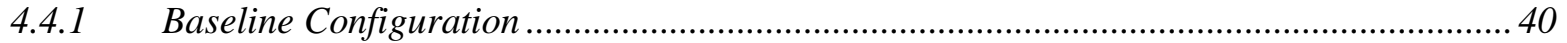

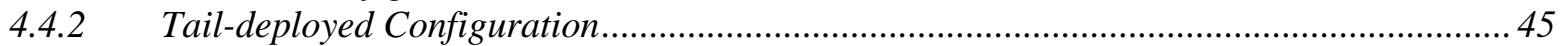

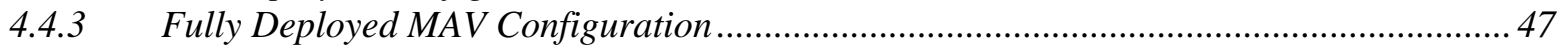

Chapter 5 Initialization Requirements for Numerical Flight Testing ............................................52

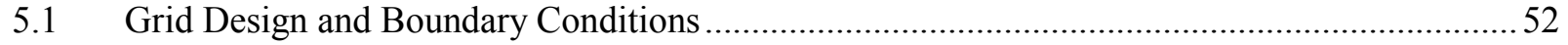

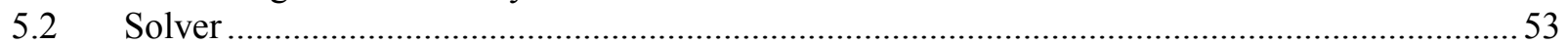

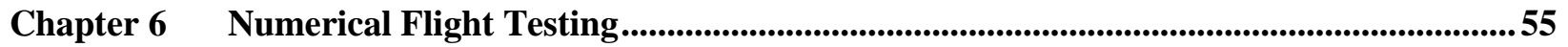

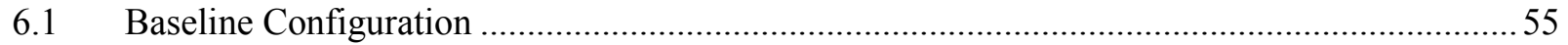

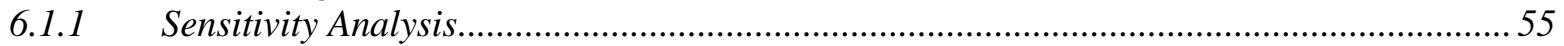

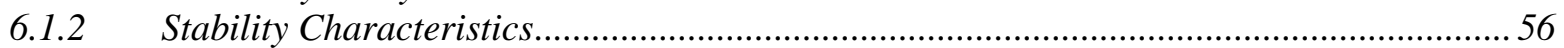

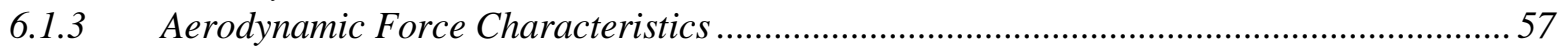

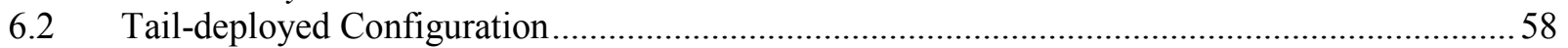

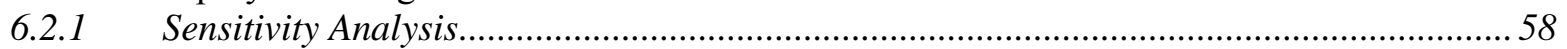

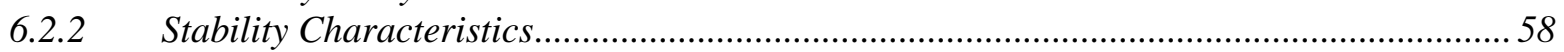

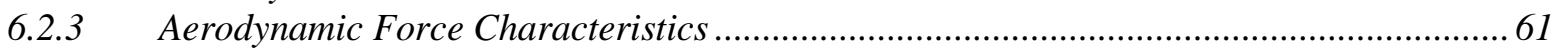

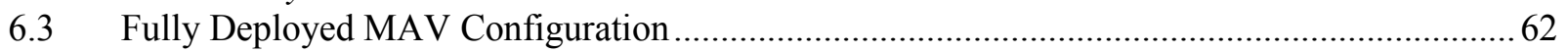




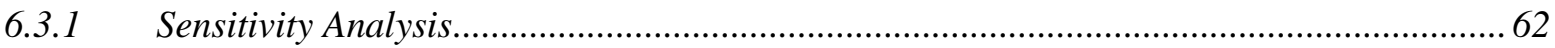

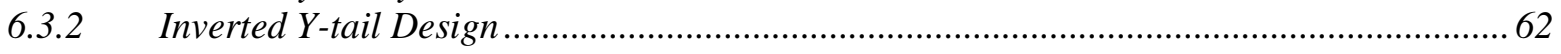

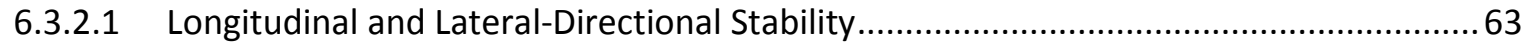

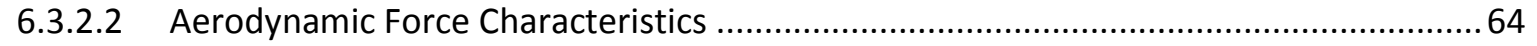

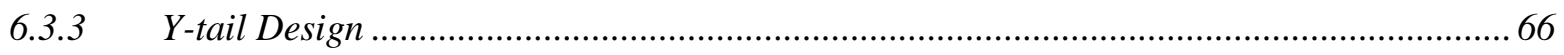

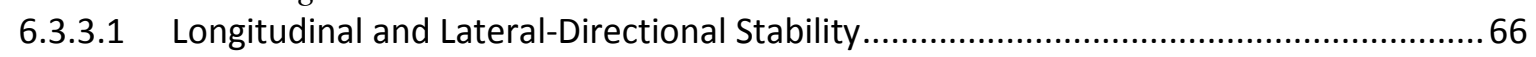

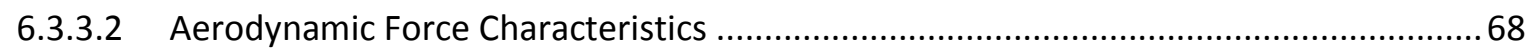

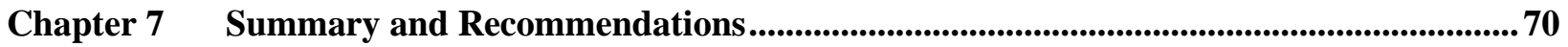

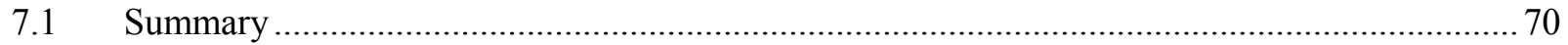

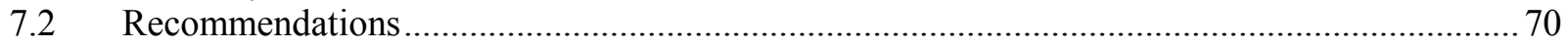

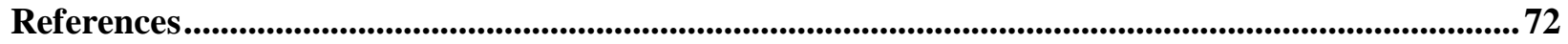

Appendix A User Defined Functions for Free Body Motion Analysis ...................................................... 74

Appendix B Configuration Details for 6-DOF Solver .................................................................................79

Appendix C Curriculum Vitae .........................................................................................................................8 


\section{List of Figures}

Figure 1-1: Dimensions of Baseline Round for HP System ........................................................... 1

Figure 1-2: M203 Grenade Launcher Under-slung on M4-Tatical Rifle, Courtesy of RM Equipment .......2

Figure 1-3: Fin Deployed Configuration for $40 \mathrm{~mm}$ Design..............................................................

Figure 1-4: MAV Configuration Showing Deployed Wing and Inverted-Y Tail.................................... 3

Figure 1-5: Simplified Model of Fin-stabilized Projectile...................................................................... 3

Figure 1-6: Simplified Model of MAV Showing Fully Deployed Wing and Tail..................................... 4

Figure 2-1: Force Components for Aerodynamic Bodies (Anderson Jr, 2007) ........................................ 5

Figure 2-2: Body-fixed Coordinate System for Baseline Configuration Illustrating Total Angle of Attack 8

Figure 2-3: Body-fixed Coordinate System for Wings-Deployed Configuration....................................... 8

Figure 2-4: Flowchart of CFD Simulation Coupled to 6-DOF Model for Numerical Flight Testing......... 12

Figure 2-5: 40 mm Subsonic Projectile Round (a) Geometry and (b) Computational Grid ...................... 13

Figure 2-6: 40 mm Subsonic Projectile Round (a) Pitching and (b) Yawing Motion............................... 13

Figure 2-7: 25 mm Ojive-cylinder-finned Projectile (a) Geometry and (b) Computational Grid............... 14

Figure 2-8: Numerical and Experimental Data for (a) Euler Pitch Attitude Angle and (b) Euler Yaw Angle

Figure 2-9: Motion History of Projectile Viewed from the Rear Looking Downrange using (a)

Computational Approach and (b) Experimental Approach ...................................................... 15

Figure 2-10: Numerical and Experimental Data for (a) Total Angle of Attack and (b) Aerodynamic Forces

Figure 2-11: F/A-18C Showing (a) GBU-31 Mounted to Left Wing Pylon and (b) Cut Plane through

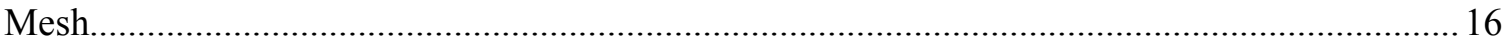

Figure 2-12: Results of (a) Distance Traveled and (b) Angular Position after JDAM Store Separation .... 17

Figure 2-13: Results of Angular Rates about Body Axes after JDAM Store Separation ......................... 17

Figure 2-14: Predicted Flight Trajectory of JDAM Store with Plotting of Surface Pressure Contours...... 17

Figure 2-15: Pathlines Colored by Velocity Magnitude Showing Recirculation Region Aft of Original

Baseline Design using Standard k- $\omega$ Model .............................................................................. 18

Figure 2-16: $40 \mathrm{~mm}$ Baseline Showing CG Location and Polar and Transverse Moments of Inertia. ....... 19

Figure 2-17: Computational and Experimental Methods Showing Inconsistent Data for Magnus Moment

Coefficient in Sub-sonic Flow Regime (DeSpirito and Heavey, 2006) ......................................20

Figure 2-18: Mach Number Contours on Pitch Plane at Mach 0.3 using Time-accurate DES Model .......21

Figure 2-19: Magnus Moment Coefficient at Subsonic, Transonic, and Supersonic Flow Regimes for $3^{\circ}$

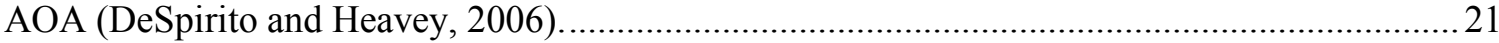

Figure 2-20: $40 \mathrm{~mm}$ Round Design Attached to Sting (Valyou and Marzocca, 2011)..........................22

Figure 2-21: Experimental Results of De-spin for $40 \mathrm{~mm}$ Projectile (Valyou and Marzocca, 2011).........22

Figure 2-22: Time Histories of (a) Composite Force and (b) Composite Moment for Spinning $40 \mathrm{~mm}$

Round at Free Stream Velocity of $62.5 \mathrm{~m} / \mathrm{s}$ and $11^{\circ}$ AOA (Valyou and Marzocca, 2011)..........22

Figure 2-23: Regions of Dynamic Stability and Dynamic Instability for Spinning Projectiles ..................23

Figure 2-24: Pitching and Yawing Motion of $40 \mathrm{~mm}$ Nonlethal Cartridge (Lyon, 1997)........................2 24

Figure 3-1: Geometric Design of Flow Domain in ICEM CFD for conducting Fixed Body CFD

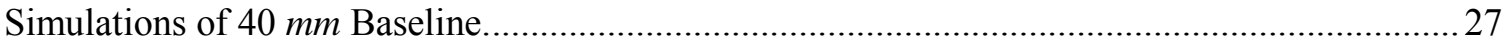

Figure 3-2: Medium Resolution Mesh of $40 \mathrm{~mm}$ Baseline and Inner Fluid Domain................................28

Figure 3-3: Mesh Wrapped around $40 \mathrm{~mm}$ Obturator Seats and Rear Casing. .......................................29

Figure 3-4: (a) Fluid Domains for $40 \mathrm{~mm}$ Baseline showing Velocity Inlet in Green and Pressure Outlet in

Red; (b) Inner Fluid Domain Magnified to Show Unstructured Cells Growing Outward from

Non-conformal Interface

Figure 3-5: (a) Inner and Outer Fluid Domains for the Tail-deployed Configuration of the $40 \mathrm{~mm}$

Projectile showing Velocity Inlet in Green and Pressure Outlet in Red; (b) Inner Fluid Domain

Magnified to Show Unstructured Cells Growing Outward from Non-conformal Interface..... 30 
Figure 3-6: Medium Resolution Mesh of Fin-stabilized $40 \mathrm{~mm}$ Projectile.

Figure 3-7: $40 \mathrm{~mm}$ Projectile Viewed from the Front Showing Mesh Wrapping around Body and Tail

Fins.

Figure 3-8: Medium Resolution Mesh of Wings-Deployed Configuration. .......................................... 31

Figure 3-9: Wings-Deployed Configuration Showing Mesh Wrapped around Body and Wing................31

Figure 3-10: Fully Deployed MAV Magnified to Show C-grid Wrapped around Wing........................... 32

Figure 3-11: Inverted Y-tail Design of Wings-Deployed Configuration Showing Mesh Wrapped

Lengthwise around Body.

Figure 3-12: (a) Medium Resolution Mesh for Fully Deployed MAV; (b) MAV Viewed from the Front

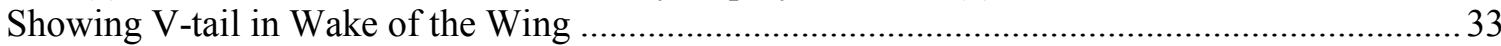

Figure 3-13: Y-tail Design of Fully Deployed MAV Configuration Showing Mesh Wrapped Lengthwise

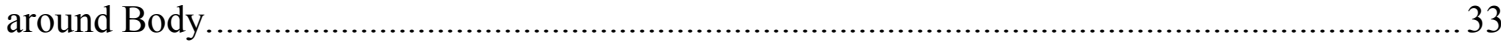

Figure 4-1: Wall $\boldsymbol{y}+$ Plotted against the Lengthwise Distance of the Baseline Configuration ................. 35

Figure 4-2: Grid Independence for Lift Data on Baseline Configuration .................................................36

Figure 4-3: Grid Independence for Drag Data on Baseline Configuration ..............................................36

Figure 4-4: Grid Independence for Lift Data on Tail-deployed Configuration ........................................ 37

Figure 4-5: Grid Independence for Drag Data on Tail-deployed Configuration .................................... 38

Figure 4-6: Wall $\boldsymbol{y}+$ Plotted against the Lengthwise Distance of the Tail-deployed Configuration.......... 38

Figure 4-7: Grid Independence for Lift Data on Fully Deployed MAV Configuration............................. 39

Figure 4-8: Grid Independence for Drag Data on Fully Deployed MAV Configuration........................... 39

Figure 4-9: Wall $\boldsymbol{y}+$ Plotted against the Lengthwise Distance of the Fully Deployed MAV Configuration

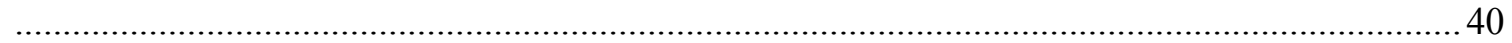

Figure 4-10: Comparison of Aerodynamic Lift for $40 \mathrm{~mm}$ Baseline Configuration.................................. 41

Figure 4-11: Comparison of Aerodynamic Drag for $40 \mathrm{~mm}$ Baseline Configuration................................ 41

Figure 4-12: Illustration of Aerodynamic Forces and Moments Acting on $40 \mathrm{~mm}$ Round....................... 41

Figure 4-13: Experimental and Computational Comparison of Pitching Moment about CG of $40 \mathrm{~mm}$

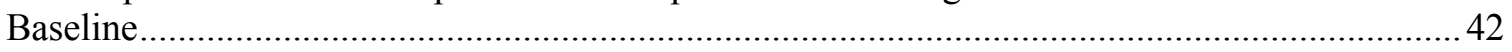

Figure 4-14: Contours of Velocity Magnitude for $40 \mathrm{~mm}$ Round at $0^{\circ}$ AOA..........................................43

Figure 4-15: Contours of Pressure Coefficient for $40 \mathrm{~mm}$ Round at $0^{\circ} \mathrm{AOA}$......................................... 43

Figure 4-16: Velocity Pathlines Showing Recirculation in Obturator Groove and Rear Casing ............... 43

Figure 4-17: Velocity Pathlines Showing Recirculation Regions Aft of the $40 \mathrm{~mm}$ Round..................... 44

Figure 4-18: Contours of Pressure Coefficient for $40 \mathrm{~mm}$ Round at $6^{\circ} \mathrm{AOA}$........................................ 44

Figure 4-19: Velocity Pathlines Showing Recirculating Flow Aft of $40 \mathrm{~mm}$ Round for $6^{\circ}$ AOA.............. 44

Figure 4-20: CFD Predictions of Aerodynamic Lift and (b) Drag on Tail-deployed Configuration.......... 45

Figure 4-21: Aerodynamic Pitching Moment for Tail-deployed Configuration...................................... 45

Figure 4-22: Contours of Velocity Magnitude for Tail-deployed Configuration at $0^{\circ} \mathrm{AOA}$..................... 46

Figure 4-23: Contours of Pressure Coefficient for Tail-deployed Configuration at $0^{\circ} \mathrm{AOA}$..................... 46

Figure 4-24: Velocity Pathlines Showing Recirculation Regions Aft of Tail-deployed Configuration at $0^{\circ}$

AOA

Figure 4-25: Velocity Pathlines Showing Recirculation Regions Aft of Tail-deployed Configuration at $6^{\circ}$

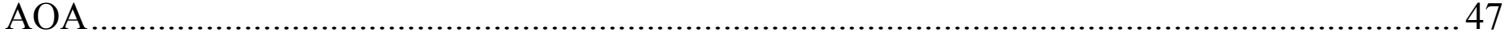

Figure 4-26: CFD Predicted (a) Aerodynamic Drag and (b) Lift-to-Drag for Inverted Y-tail Design ....... 48

Figure 4-27: Contours of Velocity Magnitude for Inverted Y-tail Configuration at $0^{\circ}$ AOA ....................48

Figure 4-28: Contours of Pressure Coefficient for Inverted-Y Tail Configuration at $0^{\circ} \mathrm{AOA} \mathrm{.................} 49$

Figure 4-29: Pathlines of Velocity Magnitude Showing Recirculation behind Inverted Y-tail of Fully

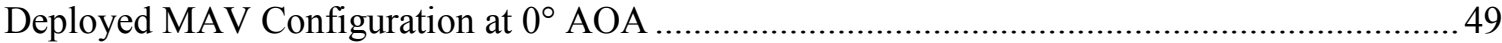

Figure 4-30: Pathlines of Velocity Magnitude Showing Recirculation behind Inverted Y-tail of Fully

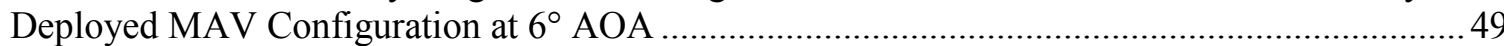

Figure 4-31: Moment Coefficient about CG of Inverted Y-tail with Variation in Tail Deflection Angle.. 50

Figure 4-32: Lift-to-Drag Ratio for Y-tail Design of Fully Deployed MAV Configuration Showing Body

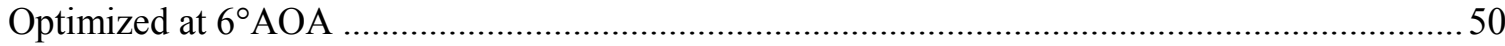


Figure 4-33: Pathlines of Velocity Magnitude Passing over Y-tail Configuration at $6^{\circ}$ AOA..................51 Figure 4-34: Moment Coefficient about CG for Y-tail Design with Variation in Tail Deflection Angle ...51 Figure 5-1: Hybrid Mesh Showing (a) Farfield and Outer Fluid Domain for Simulating Free Body Motion of Tail-deployed Configuration and (b) Inner Fluid Domain Magnified to Show Unstructured Cells Growing Outward from Non-conformal Interface... 52

Figure 5-2: Hybrid Mesh Showing (a) Farfield and Outer Fluid Domain for Simulating Free Body Motion of Fully Deployed MAV Configuration and (b) Inner Fluid Domain Magnified to Show Unstructured Cells Growing Outward from Non-conformal Interface ........................................53

Figure 6-1: Sensitivity Analysis on Baseline Configuration for Numerical Flight Testing.......................55

Figure 6-2: Numerical Flight Test Results of $40 \mathrm{~mm}$ Baseline Round (a) $\alpha-\beta$ Curve for $0.35 \mathrm{sec}$ and ......56 Figure 6-3: Velocity Contours of $40 \mathrm{~mm}$ Baseline Configuration at 0.1 Second after Launch ..................57 Figure 6-4: $40 \mathrm{~mm}$ Baseline Configuration Coefficient Magnitudes of (a) Aerodynamic Lift and (b) Drag

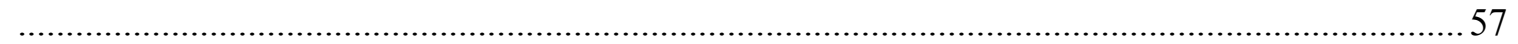

Figure 6-5: Sensitivity Analysis of Tail-deployed Configuration for Numerical Flight Testing...............58

Figure 6-6: Tail-deployed Configuration Showing (a) Spin Rate Decay and (b) and Velocity Magnitude59 Figure 6-7: Tail-deployed Configuration Showing (a) Dampening of Longitudinal and (b) Lateral Motion

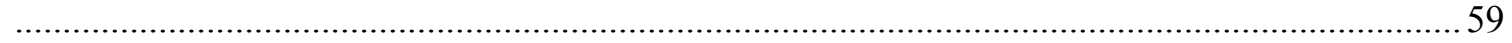

Figure 6-8: Tail-deployed Configuration Showing (a) $\alpha-\beta$ curve and (b) Total Angle of Attack..............59

Figure 6-9: Velocity Contours of Tail-deployed Configuration Showing Wake Flow Immediately ..........60 Figure 6-10: Velocity Contours of Tail-deployed Configuration Showing Vortex Shedding Immediately

Figure 6-11: Velocity Contours of Fin-stabilized Projectile at 0.5 Second after Deployment of Tail ....... 61 Figure 6-12: Figure 6-12: Velocity Contours of Fin-stabilized Projectile Showing Wake Flow ...............61 Figure 6-13: Aerodynamic Results for (a) Lift and (b) Drag on Tail-deployed Configuration .................. 62 Figure 6-14: Sensitivity Analysis of Fully Deployed MAV Configuration for Numerical Flight Testing. 62 Figure 6-15: Velocity Magnitude for Inverted Y-tail Design of Fully Deployed MAV Configuration ......63 Figure 6-16: Longitudinal Dynamics of Inverted Y-tail Configuration (a) Angle of Attack and Pitch

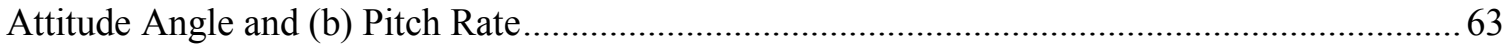

Figure 6-17: Lateral Dynamics of Inverted Y-tail Configuration (a) Bank Angle and (b) Roll Rate.........64 Figure 6-18: Lateral Dynamics of Inverted Y-tail Configuration (a) Heading Angle and (b) Yaw Rate ... 64 Figure 6-19: Angle of Sideslip for Inverted Y-tail Configuration ........................................................ 64 Figure 6-20: Inverted Y-tail Configuration Data for (a) Aerodynamic Drag and (b) Lift-to-Drag ............ 65 Figure 6-21: Velocity Contours of Inverted Y-tail Configuration Showing Wake Flow Immediately ....... 65 Figure 6-22: Velocity Contours Showing Wingtip Vorticity for Inverted Y-tail Configuration Immediately

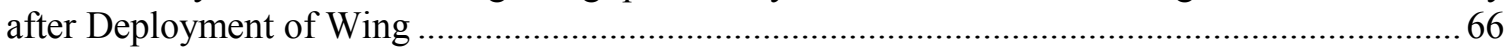

Figure 6-23: Velocity Contours Showing Wingtip Vorticity for Inverted Y-tail Configuration .................66

Figure 6-24: Longitudinal Dynamics of Y-tail Configuration for 7.5 $5^{\circ}$ Tail Deflection Showing ..............67

Figure 6-25: Longitudinal Dynamics of Y-tail Configuration for $0^{\circ}$ Tail Deflection Showing (a) Pitch

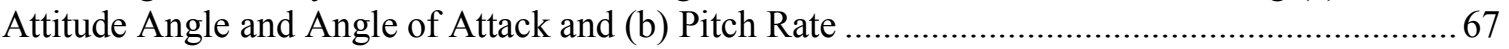

Figure 6-26: Figure 6-29: Lateral Dynamics of Y-tail Configuration Showing Motion History of ............ 68

Figure 6-27: Y-tail Configuration Data for (a) Aerodynamic Drag and (b) Lift-to-Drag......................... 68

Figure 6-28: Velocity Contours of Y-tail Configuration Showing Wake Flow Immediately after ............ 69

Figure 6-29: Velocity Pathlines of Y-tail Configuration Showing Wing Tip Vorticity Immediately after

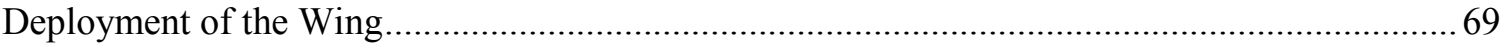

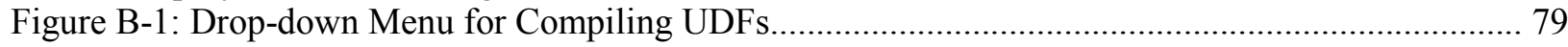

Figure B-2: UDFs Added to Source File List to be Compiled and Loaded............................................ 79

Figure B-3: UDF Hooked to Execute at End Function to Compute Forces and Moments.........................80

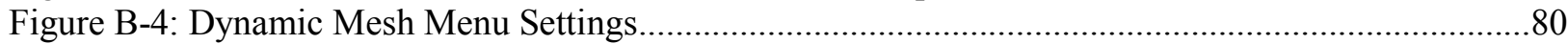

Figure B-5: Configuration Settings for (a) Smoothing and (b) Remeshing Parameters.......................... 81 
Figure B-6: Dynamic Mesh Zone Configurations for Initial Simulation................................................82

Figure B-7: Configuration Settings for (a) Dynamic Mesh Setup and (b) 6-DOF Options........................82

Figure B-8: Dynamic Mesh Zone Configurations Settings for 6-DOF Simulation...................................83 


\section{List of Tables}

Table 2-1: Initial Parameters for Two Flight Test Simulations on $25 \mathrm{~mm}$ Projectile .............................. 14

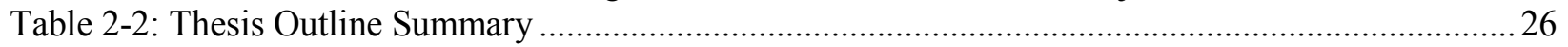

Table 4-1: Lift and Drag Data for Grid Independence Study of Baseline Configuration ...........................36

Table 4-2: Lift and Drag Data for Grid Independence Study of Tail-deployed Configuration ...................37

Table 4-3: Lift and Drag Data for Grid Independence Study of Fully Deployed MAV Configuration ......39

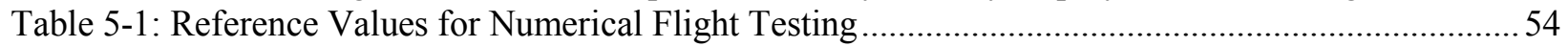

Table 5-2: Initialization Parameters for Numerical Flight Testing ........................................................54

Table 6-1: Moments and Products of Inertia Properties for Baseline, Tail-deployed, and Wings-Deployed

Configurations Experiencing Free Body Motion................................................................... 55 


\section{List of Symbols}

Symbol

a

C

$C_{A}$

$C_{D}$

$C_{L}$

$C_{L_{\alpha}}$

$C_{l}$

$C_{l_{p}}$

$C_{m}$

$C_{m_{C G}}$

$C_{m_{\text {nose }}}$

$C_{m_{\alpha}}$

$C_{m_{p \alpha}}$

$\left(C_{m_{q}}+C_{m_{\dot{\alpha}}}\right)$

$C_{N_{\alpha_{f i n}}}$

$C_{N_{\alpha_{\text {fin,root }}}}$

$C_{n}$

$C_{Y}$

c

D

d

$F_{A}, F_{Y}, F_{N}$

$F_{1}, F_{2}, F_{3}$

$g$

$I_{x x}, I_{y y}, I_{z z}$
Tail fin span

\section{Description}

\section{Units}

Courant number

Axial force coefficient

Drag coefficient

Lift coefficient

Change in lift coefficient with angle of attack

Rolling moment coefficient

Spin damping moment coefficient

Pitching moment coefficient

Pitching moment coefficient at center of gravity

Pitching moment coefficient at nose of projectile body

Pitching moment coefficient derivative

Magnus moment coefficient

Pitch damping moment coefficient

Normal force coefficient on tail fin

Normal force coefficient at root of tail fin

Yawing moment coefficient

Side force coefficient

Chord length of tail fin

$m$

Drag

$\mathrm{N}$

Reference length of projectile body

$m$

$\mathrm{N}$

$\mathrm{N}$

Acceleration due to gravity

$\mathrm{m} / \mathrm{s}^{2}$

Moments of inertia about airplane roll, pitch, and yaw axes $\mathrm{kg} \cdot \mathrm{m}^{2}$ 


\section{Symbol}

$I_{x y}, I_{x z}, I_{y z}$

$I_{P}$

$I_{T}$

$\mathrm{k}$

$L$

$L_{A}$

$l$

$M_{A}$

$m$

$N_{A}$

$\mathcal{N}$

$P, Q, R$

$\dot{P}, \dot{Q}, \dot{R}$

$P_{i}$

$q$

$r_{0}$

$S$

$S_{d}$

$S_{g}$

$t$

$u, v, w$

$\dot{u}, \dot{v}, \dot{w}$

$u^{*}$

V

$V_{1}, V_{2}, V_{3}$

$u_{0}, v_{0}, w_{0}$

$\dot{V}_{1}, \dot{V}_{2}, \dot{V}_{3}$

$x$

$x_{1}, x_{2}, x_{3}$

\section{Description}

Airplane products of inertia

Polar moment of inertia for symmetric projectiles

Transverse moment of inertia for symmetric projectiles

Turbulent kinetic energy

Lift

Rolling moment due to aerodynamic force

Distance between projectile nose and center of gravity location

Pitching moment due to aerodynamic force

Mass of aerodynamic body

Yawing moment due to aerodynamic force

Number of tail fins

Angular velocity about roll, pitch, and yaw axes, respectively

Time rate of change of angular velocity about roll, pitch, and yaw axes, respectively

Spin rate of projectile exiting muzzle of weapon system

Dynamic pressure

Radius of projectile body

Reference cross-sectional area of projectile body

Dynamic stability factor

Gyroscopic stability factor

Time

Linear velocity components at CG along body axes

Time rate of change of linear velocity at CG along body axes

Friction velocity

Free stream (reference) velocity

Linear velocity components at CG in global coordinates

Velocity components at $\mathrm{CG}$ in global coordinates when projectile exits muzzle of weapon system

Time rate of change of linear momentum components at CG in global coordinates

Average grid spacing on surface of aerodynamic body

Position components at $\mathrm{CG}$ in global coordinates

\section{Units}

$\mathrm{kg} \cdot \mathrm{m}^{2}$

$\mathrm{kg} \cdot \mathrm{m}^{2}$

$\mathrm{kg} \cdot \mathrm{m}^{2}$

$\mathrm{m}^{2} / \mathrm{s}^{2}$

$\mathrm{N}$

$N \cdot m$

m

$N \cdot m$

$k g$

$N \cdot m$

$\frac{\mathrm{rad}}{\mathrm{s}}$ or $\frac{\mathrm{deg}}{\mathrm{s}}$

$\frac{\mathrm{rad}}{\mathrm{s}^{2}}$ or $\frac{\mathrm{deg}}{\mathrm{s}^{2}}$

$\mathrm{rad} / \mathrm{s}$

$N / m^{2}$

m

$m^{2}$

sec

$\mathrm{m} / \mathrm{s}$

$\mathrm{m} / \mathrm{s}^{2}$

$\mathrm{m} / \mathrm{s}$

$\mathrm{m} / \mathrm{s}$

$\mathrm{m} / \mathrm{s}$

$\mathrm{m} / \mathrm{s}$

$\mathrm{m} / \mathrm{s}$

m

m 
Symbol

$y$

$y^{+}$

$\alpha$

$\alpha_{t}$

$\beta$

$\delta_{F}$

$\theta$

$\dot{\theta}$

$\theta_{0}$

$\mu$

$v$

$\pi$

$\rho$

$\phi$

$\dot{\phi}$

$\psi$

$\dot{\psi}$

$\psi_{0}$

$\omega$

\section{Description}

Distance between the first and second grid points off the boundary wall

Dimensionless distance off the wall boundary

Angle of attack

Total angle of attack for symmetric projectiles

Angle of Sideslip

Fin cant angle

Pitch attitude angle

Time rate of change of pitch attitude angle

Pitch attitude angle of projectile at launch

Dynamic viscosity of air

Kinematic viscosity of air

Mathematical constant

Air density

Bank angle

Time rate of change of bank angle

Heading angle

Time rate of change of heading angle

Projectile heading angle at launch

Specific dissipation rate

\section{Units}

$m$

rad or deg

rad or deg

rad or deg

rad or deg

rad or deg

$\frac{\mathrm{rad}}{\mathrm{s}}$ or $\frac{\mathrm{deg}}{\mathrm{s}}$

rad or deg

$\mathrm{kg} /(\mathrm{m} \cdot \mathrm{s})$

$\mathrm{m}^{2} / \mathrm{s}$

$$
\mathrm{kg} / \mathrm{m}^{3}
$$

rad or deg

$\frac{\mathrm{rad}}{\mathrm{s}}$ or $\frac{\mathrm{deg}}{\mathrm{s}}$

rad or deg

$\frac{\mathrm{rad}}{\mathrm{s}}$ or $\frac{\mathrm{deg}}{\mathrm{s}}$

rad or deg

$1 / s$ 


\section{Chapter 1 Problem Statement}

\subsection{Problem Description}

A new type of micro aerial vehicles (MAVs) currently in development by the US Army Armaments Research Development and Engineering Center (ARDEC) are known as hybrid projectile (HP) rounds. Some of the types of rounds that are in existence today have deployable fins for guidance. In contrast, the HP rounds are designed to transform into a miniaturized aircraft for the purpose of obtaining performance improvements in both range and accuracy. This new MAV design consists of a two-stage deployment process of its control surfaces in which the tail fins are first deployed immediately after being launched from a tube system followed by deployment of the wings at the apex of the original ballistic trajectory. With that being said, research in HP technology was performed that involved the development of a baseline configuration consisting of internal compactable components, such as the wing and tail assembly mechanisms. Because the projectile would transform from ballistic flight to aircraft flight, it was necessary to perform an analysis of its aerodynamic behavior for the baseline, fin deployed, and fully deployed MAV configurations. This was accomplished by incorporating a relatively new simulation tool that couples a computational fluid dynamics (CFD) analysis with Six-degree-of-freedom (6-DOF) flight prediction. This coupling allowed the numerical prediction for the aerodynamic flight behavior in free flight motion. With this information, conclusions could be drawn from the longitudinal and lateral-directional stability of the MAV.

The HP design specifications for WVU were to use a M203 grenade launcher or its replacement, the M302, to launch a $40 \mathrm{~mm}$ round at a $45^{\circ}$ angle relative to the ground at a muzzle velocity of $70 \mathrm{~m} / \mathrm{s}$ and a spin rate of $60 \mathrm{~Hz}$ or 3,600 RPM. The mass of the $40 \mathrm{~mm}$ round, including all internal components, was approximately $180 \mathrm{~g}$. The round consists of an obturator that includes an annular ring having an inner surface in contact with the shaped surface of the annular groove and an outer surface in contact with the inner surface of the bore of the tube system. The function of the obturator groove is to restrict the flow of charge gases from moving forward of the projectile when launched from the tube system. In order for the round to be compatible with the M203 grenade launcher, the cylindrical length could be no greater than $133 \mathrm{~mm}$. Therefore, the length of the round was increased to the maximum allowable from the original 80 $\mathrm{mm}$ length design thereby increasing the amount of space for additional equipment necessary for the HP system. As shown in Figure 1-1, a computer aided design (CAD) drawing of the $40 \mathrm{~mm}$ round is provided that details the hemispherical nose, obturator groove, and aft steps. Figure 1-2 presents an M203 grenade launcher mounted to an M4-Tatical rifle, which could be used as the weapon system to launch the $40 \mathrm{~mm}$ HP rounds.

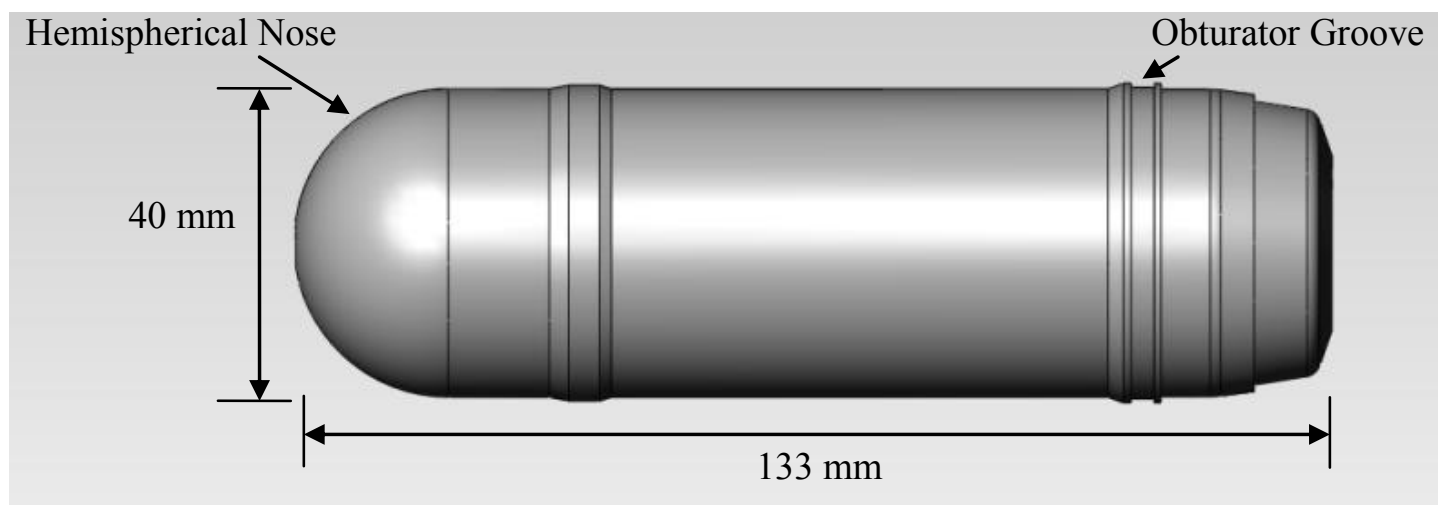

Figure 1-1: Dimensions of Baseline Round for HP System 


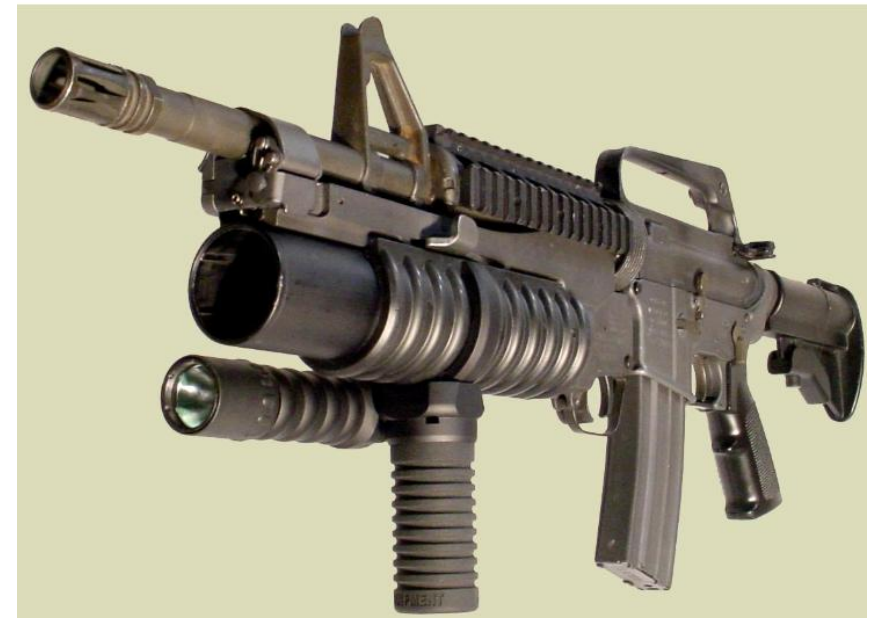

Figure 1-2: M203 Grenade Launcher Under-slung on M4-Tatical Rifle, Courtesy of RM Equipment

Once the round had been launched, the design was such that the rear casing would separate from the main body followed by deployment of the tail fins for stability and to ensure that the projectile would have despun before deploying its wings. Due to volume restrictions, the tail design was limited to three fins. Therefore, the two possible designs to consider were the Y-tail and inverted Y-tail configurations. The tail fin geometry consisted of a rectangular planform area of approximately $1,300 \mathrm{~mm}^{2}$. Figure $1-3$ shows a representation of the fin-stabilized $40 \mathrm{~mm}$ projectile.

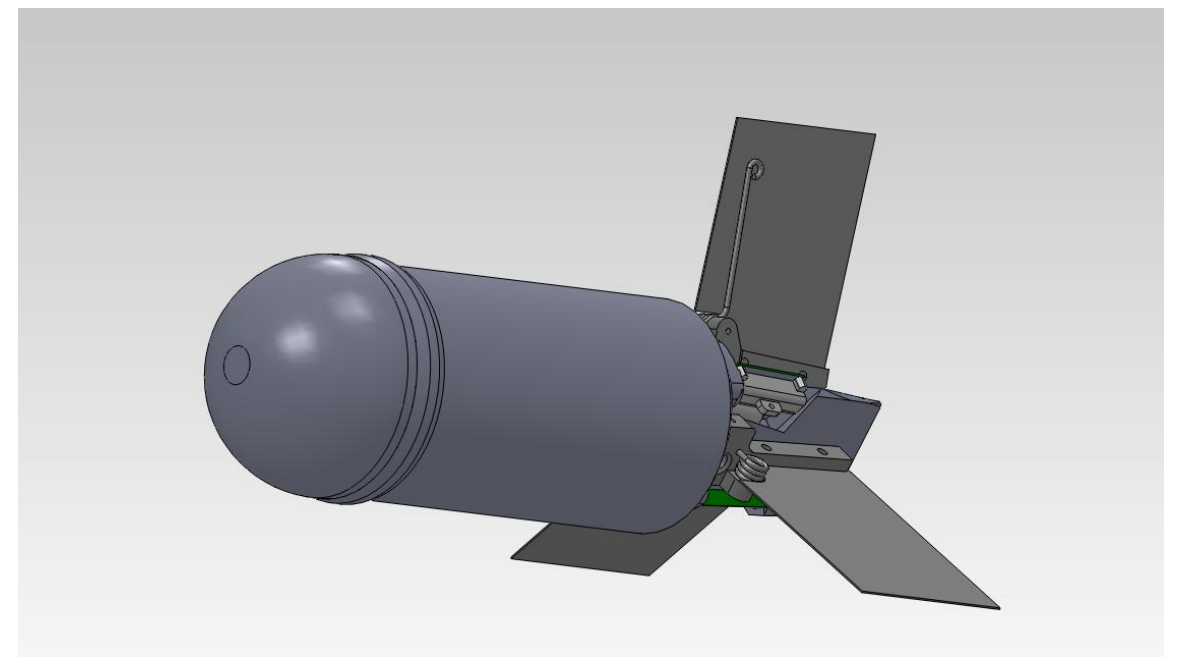

Figure 1-3: Fin Deployed Configuration for $40 \mathrm{~mm}$ Design.

As the fin-stabilized projectile reached the apex of the trajectory, it would deploy the wing for the purpose of increasing its maximum range and flight time. At the instant the wing was to be deployed, the velocity of the MAV should be approximately $45 \mathrm{~m} / \mathrm{s}$, according to the simple air trajectory analysis performed by Hamburg (2010). Similar to the tail control surfaces, the wing had a rectangular planform area of approximately $7,750 \mathrm{~mm}^{2}$. It should be noted that the final design of the wing and tail assembly is to use a flexible composite material, but for the purposes of this research study, the tail fins were assumed to be a rigid structure. This would avoid additional computational resources that would require a system coupling between a CFD and finite element analysis (FEA) solvers to perform a simulation of a fluidstructure interaction. Figure 1-4 provides a CAD model of the fully deployed MAV that incorporates the inverted Y-tail configuration. 


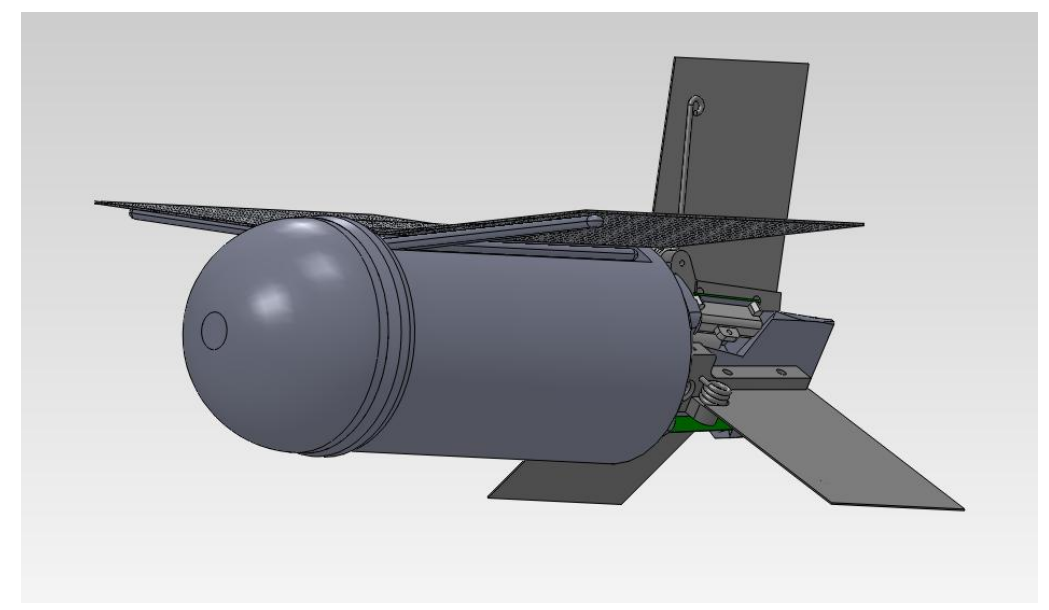

Figure 1-4: MAV Configuration Showing Deployed Wing and Inverted-Y Tail.

\subsection{Approach and Overview of Work}

The primary objective of this research study was to obtain a detailed time history of the aerodynamic and flight behavior for various configurations of the $40 \mathrm{~mm} \mathrm{HP}$ in which several simulations were performed. This was accomplished by incorporating a relatively new simulation tool that couples a computational fluid dynamics (CFD) analysis with 6-DOF flight prediction. This coupling allowed for the MAV's aerodynamic flight behavior to be numerically predicted while undergoing free flight motion. Therefore, this analysis is being called numerical flight testing. Prior to any simulations, it was necessary to generate a three-dimensional mesh for each of the models and perform a grid independence study. As shown in Figure 1-3 and Figure 1-4, the complexity in meshing the aerodynamic body including the control surface mechanisms would require a substantial amount of time and effort. Therefore, the models were reconstructed such that the aft end was simplified thereby eliminating the mechanisms used to deploy the tail fins. The fully deployed MAV configuration was also simplified in which the mechanism for deploying the wing was removed as well. Figure 1-5 and Figure 1-6 show the simplified models for the tail-deployed and fully deployed MAV configurations, respectively.

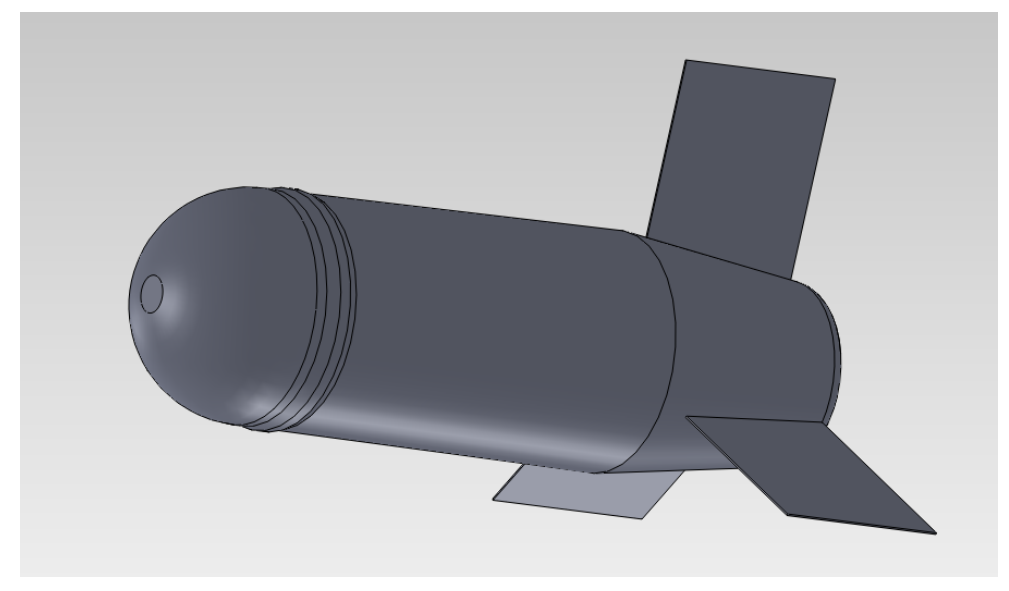

Figure 1-5: Simplified Model of Fin-stabilized Projectile. 


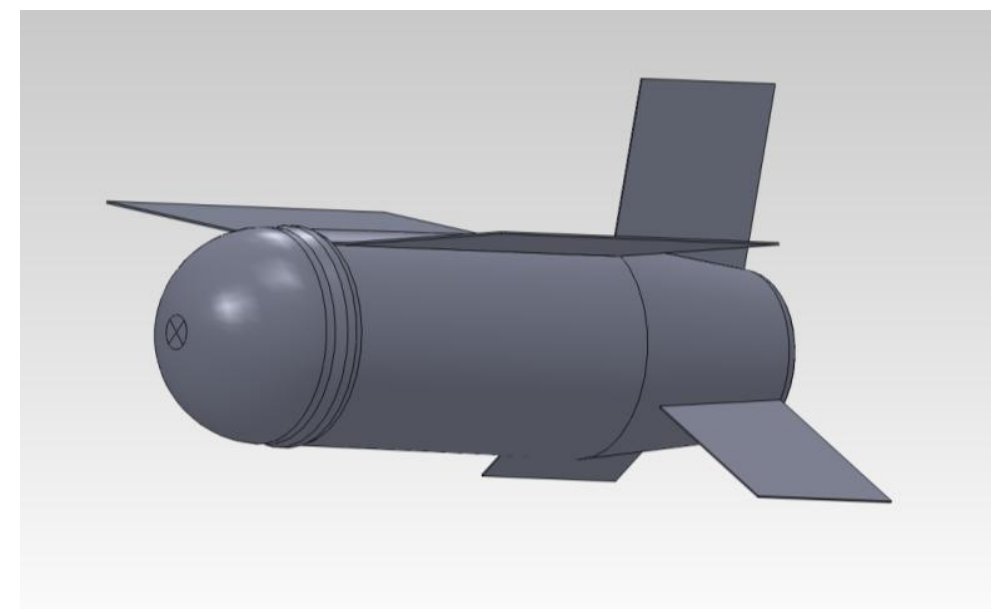

Figure 1-6: Simplified Model of MAV Showing Fully Deployed Wing and Tail.

Once a sufficient mesh quality was attained for each of the configurations, several, more traditional, CFD simulations were conducted in which the airflow traveled relative to the fixed body. The aerodynamic lift, drag, and pitching moment were obtained for a range of angles of attack. In order to perform a simulation undergoing free body motion, knowledge of the center of gravity (CG) location was required. For this reason, the tail on the fully deployed MAV had to be trimmed in order to determine the placement of the CG.

Following these aerodynamic simulations, numerical flight testing was performed for the various configurations in the absolute reference frame. The baseline and fin-stabilized projectiles were executed at launch to simulate the MAV's dynamic stability along its original ballistic trajectory for a $45^{\circ}$ launch angle at a velocity of $70 \mathrm{~m} / \mathrm{s}$, whereas the fully deployed MAV was executed at the apex of the trajectory to simulate its longitudinal and lateral-directional flight dynamics at a velocity of $45 \mathrm{~m} / \mathrm{s}$. In addition to the inverted Y-tail design, another numerical flight test was executed on the fully deployed MAV utilizing the Y-tail design. Once the data was obtained for the numerical flight tests, conclusions could be drawn from the aerodynamic force and stability characteristics of the various configurations. 


\section{Chapter 2 Background and Literature Review}

This chapter provides a review of background information and documentation that is relevant to fulfilling the objectives of this thesis report. Beginning in the first section, a brief explanation of the fundamental aerodynamic concepts is defined that includes the external forces and moments imposed on a three-dimensional body. In the following section, the concept of numerical flight testing will discuss how the information is passed between the tightly coupled CFD simulation and 6-DOF flight dynamics model to predict the translational and rotational motion of the tube-launched transformable MAV. The 6-DOF equations will require the external forces and moments that are presented in the first section. Additionally, this section provides prior studies utilizing such a coupled system before proceeding with the current research. Because this coupled system relies heavily on CFD, it is necessary to provide documentation explaining the reasoning behind selecting the aerodynamic model that best describes the physics of the airflow. Since the MAV is to be launched out of a tube in its baseline configuration, a basic understanding of ballistics is essential to this research. Prior studies on gyroscopic and dynamic stability of spin stabilized projectiles will be discussed. Additionally, de-spin for fin-stabilized projectiles will be discussed as well.

\subsection{Development of MAV Aerodynamics}

A major requirement for this research study involved analyzing the longitudinal and lateraldirectional stability of a MAV. Therefore, this required a basic understanding of aircraft aerodynamic forces and moments.

\subsubsection{Aerodynamic Forces}

As explained in Anderson (2007), the forces acting on an aerodynamic body traveling through a fluid, such as air, can only be transmitted through the integration of pressure and shear stress distributions over the body surface. As shown in Figure 2-1, the resultant aerodynamic force can be divided up into lift and drag components. In three-dimensional aerodynamics, the side force is an additional component of the resultant force in which its direction is perpendicular to both the lift and drag components.

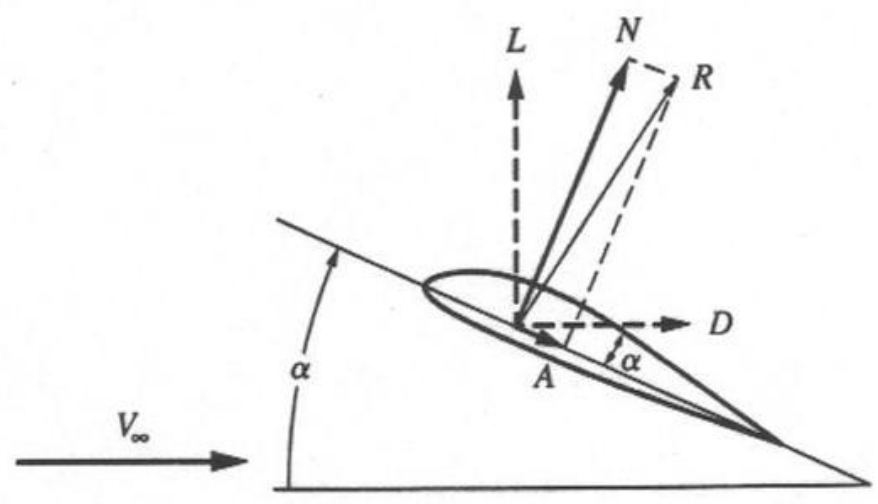

Figure 2-1: Force Components for Aerodynamic Bodies (Anderson Jr, 2007)

\subsubsection{Lift}

The force component acting on an aerodynamic body that is perpendicular to the free stream velocity vector is known as lift. This force always remains perpendicular to the velocity vector, regardless of the change in angle of attack. There is a limit to the amount of lift that an aerodynamic body can generate, which occurs when flow separation is so large that lift begins to decrease. At this point, the 
aerodynamic body has reached its critical angle of attack and is said to have stalled. A dimensionless value can be defined that relates the lift generated by an aerodynamic body called the lift coefficient, as highlighted in Equation 2-1.

$$
C_{L}=\frac{L}{\frac{1}{2} \rho V^{2} S}
$$

\subsubsection{Drag}

The force component acting on an aerodynamic body that is parallel to the free stream velocity vector is known as drag. Similar to lift, the drag force remains parallel to the velocity vector, regardless of the changes in angle of attack. In a real fluid with non-zero viscosity and a free stream subsonic velocity, the total drag acting on a body is a combination of parasitic and lift induced drag. Parasitic drag is composed of pressure drag and skin-friction drag where pressure drag is created by normal forces perpendicular to the boundary surface, and skin-friction drag is created by tangential forces parallel to the boundary surface. The other component of the total drag is called lift induced drag, which accounts for changes in the angle of attack thereby redirecting the airflow to generate lift. It should be noted that when the flow becomes detached, a difference in the pressure distribution causes parasite drag. A dimensionless value can be defined that relates the total drag generated by an aerodynamic body called the drag coefficient, as shown in Equation 2-2.

$$
C_{D}=\frac{D}{\frac{1}{2} \rho V^{2} S}
$$

\subsubsection{Normal and Axial Forces}

The lift and drag forces previously discussed are defined in the stability axes. As shown above in Figure 2-1, the normal and axial forces can be defined in the body axes in which the coordinate systems are oriented by the angle of attack, $\alpha$. Equations 2-3 and 2-4 relate the lift and drag to the normal and axial forces.

$$
\begin{aligned}
& C_{L}=C_{N} \cos \alpha-C_{A} \sin \alpha \\
& C_{D}=C_{N} \sin \alpha+C_{A} \cos \alpha
\end{aligned}
$$

\subsubsection{Side Force}

The side force component is a force that acts perpendicular to the velocity vector and is positive to the right viewed from the rear of the aircraft. This side force is collinear in both the stability and body axes. Similar to the lift and drag, a dimensionless value can be defined that relates the side force acting on an aerodynamic body called the side force coefficient, as shown in Equation 2-5.

$$
C_{Y}=\frac{F_{Y}}{\frac{1}{2} \rho V^{2} S}
$$




\subsubsection{Aerodynamic Moments}

When an aerodynamic force is applied to a body at a distance from its center of gravity location, a moment is produced. A moment can occur for a number of reasons, such as an apparent accelerated airflow over a lifting body, control surface deflection, or wind gusts. Also, it is possible for more than one moment to occur simultaneously, such as when an aerodynamic body experiences Dutch roll characteristics. If a force causes the aerodynamic body to rotate left or right about its longitudinal axis, a rolling moment is produced. By applying the right hand rule, a rolling moment is positive if the body rotates clockwise viewed from the rear. A dimensionless value can be defined that relates the rolling moment acting on an aerodynamic body called the rolling moment coefficient, as shown in Equation 2-6.

$$
C_{l}=\frac{L_{A}}{\frac{1}{2} \rho V^{2} S d}
$$

If a force causes the aerodynamic body to change its orientation above or below the horizon, a pitching moment is generated. The standard convention is such that a negative pitching moment produces a nose down orientation. If a force causes the aerodynamic body to change its directional orientation in which the nose points to the left or right of the velocity vector, a yawing moment is generated. The standard convention for a positive yawing moment is such that the nose of the aerodynamic body rotates to the right. Similarly, dimensionless values can be defined that relates the pitching and yawing moments acting on an aerodynamic body called the pitching and yawing moment coefficients, as highlighted in Equations 2-7 and 2-8, respectively.

$$
\begin{aligned}
& C_{m}=\frac{M_{A}}{\frac{1}{2} \rho V^{2} S d} \\
& C_{n}=\frac{N_{A}}{\frac{1}{2} \rho V^{2} S d}
\end{aligned}
$$

\subsection{Six-Degree-Of-Freedom Trajectories}

The method utilized in this research study to perform numerical flight tests of a tube-launched transformable MAV involved a coupling between a time accurate CFD simulation and a 6-DOF rigid body flight dynamics model. McCoy (1999) provides an overview of trajectories from the most simplistic vacuum trajectory to the more modern computationally intensive 6-DOF trajectory. The major disadvantage of these approaches, excluding 6-DOF trajectories, is their inability to obtain a complete time history of the projectile's aerodynamic motion. That is, the methods utilize a 3-DOF solver to compute its flight trajectory. The 6-DOF approach can provide the most accurate information by tracking the translational and rotational motion of the projectile as well as calculating the associated aerodynamic forces and moments. However, it comes with a high computational expense due to the six ordinary differential equations (ODEs) that it must solve simultaneously. Figure 2-2 provides an illustration of the inertial and body coordinate systems for the $40 \mathrm{~mm}$ baseline round. The term, $\alpha_{t}$, in the figure is the total angle of attack in which the text explains that modern ballisticians refer to this term as a combined pitching and yawing motion. The way this term has been defined is contrary to aircraft nomenclature because "pitch" or "angle of attack" refers to the nose pointing above or below the flight path and "yaw" or "angle of sideslip" refers to the nose pointing to the left or right of the flight path. A good approximation for the total angle of attack is provided in Equation 2-9, but it is only valid for an angle of attack, $\alpha$, or angle of sideslip, $\beta$, of less than $15^{\circ}$. 


$$
\alpha_{t} \approx \sqrt{\alpha^{2}+\beta^{2}}
$$

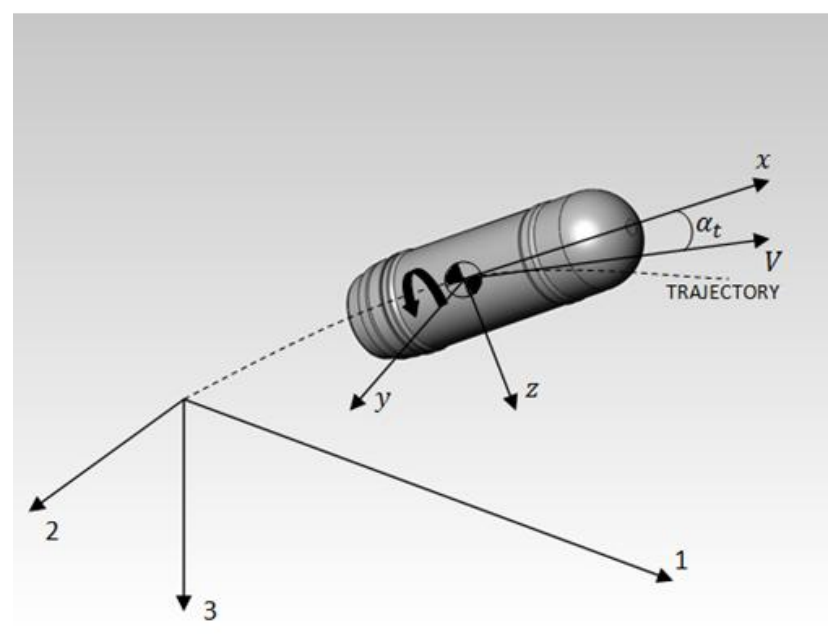

Figure 2-2: Body-fixed Coordinate System for Baseline Configuration Illustrating Total Angle of Attack

Figure 2-3 provides an illustration of the inertial and body coordinate systems for the wingsdeployed configuration. However, the total angle of attack is not of interest since the pitching and yawing dynamics of airplanes are not described by epicyclic motion. Instead, the angle of attack and angle of sideslip are analyzed individually.

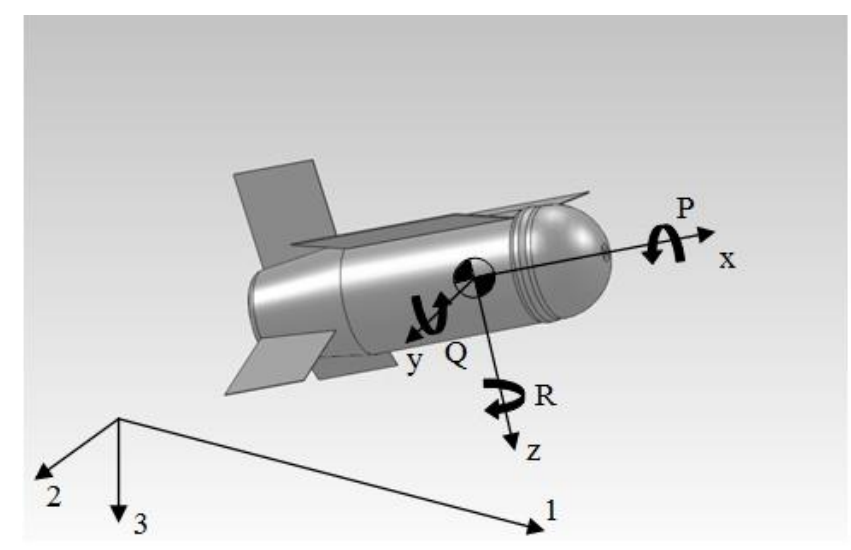

Figure 2-3: Body-fixed Coordinate System for Wings-Deployed Configuration

Roskam (2003) indicates the 6-DOF equations of motion for calculating the translational and rotational motion of an aerodynamic body. These equations are applied to the tube-launched transformable MAV in the body-fixed axis system and are presented and rewritten for use in this research study. Equation 2-10 through Equation 2-12 provides the ordinary differential equations for the forces along the axial, side, and normal directions, respectively. Equation 2-13 through Equation 2-15 provide 
the ODEs for the moments about the roll, pitch, and yaw axes, respectively. These equations require knowledge of the three translational velocities $(u, v, w)$ and the three angular velocities $(P, Q, R)$ in the body axis system.

$$
\begin{gathered}
m(\dot{u}-R v+Q w)=-m g \sin \theta-F_{A} \\
m(\dot{v}+R u-P w)=m g \sin \phi \cos \theta+F_{Y} \\
m(\dot{w}-Q u+P v)=m g \cos \phi \cos \theta-F_{N} \\
I_{x x} \dot{P}-I_{x z} \dot{R}-I_{x z} P Q+\left(I_{z z}-I_{y y}\right) R Q=L_{A} \\
I_{y y} \dot{Q}+\left(I_{x x}-I_{z z}\right) P R+I_{x z}\left(P^{2}-R^{2}\right)=M_{A} \\
I_{z z} \dot{R}-I_{x z} \dot{P}+\left(I_{y y}-I_{x x}\right) P Q+I_{x z} Q R=N_{A}
\end{gathered}
$$

The above six equations of motion can be rearranged to obtain the time rate of change of the three linear and three angular momentum equations, as highlighted in Equation 2-16 through Equation 2-21. In order to solve Equation 2-13 and Equation 2-15 for the time rate of change in roll and yaw, they must be solved simultaneously since there is a coupling in the lateral-directional dynamics. The final forms of the time rate of change in roll and yaw are presented in the work by Khalil, et. al. (2009).

$$
\begin{gathered}
\dot{u}=-\left[\overline{C_{A}}\right] V-g \sin \theta+R v-Q w \\
\dot{v}=\left[\overline{C_{Y}}\right] V+g \sin \phi \cos \theta+P w-R u \\
\dot{w}=-\left[\overline{C_{N}}\right] V+g \cos \phi \cos \theta+Q u-P v \\
\dot{P}=\frac{L_{A} I_{z z}+N_{A} I_{x z}+P Q\left(I_{x x} I_{x z}-I_{y y} I_{x z}+I_{z z} I_{x z}\right)+Q R\left(I_{y y} I_{z z}-I_{x z}^{2}-I_{z z}^{2}\right)}{I_{x x} I_{z z}-I_{x z}^{2}} \\
\dot{Q}=\frac{M_{A}+P R\left(I_{z z}-I_{x x}\right)+\left(R^{2}-P^{2}\right) I_{x z}}{I_{y y}}
\end{gathered}
$$




$$
\dot{R}=\frac{L_{A} I_{z x}+N_{A} I_{x x}+R Q\left(I_{y y} I_{x z}-I_{x x} I_{x z}-I_{z z} I_{x z}\right)+Q P\left(I_{x x}^{2}-I_{x x} I_{y y}+I_{x z}^{2}\right)}{I_{x x} I_{z z}-I_{x z}^{2}}
$$

The aerodynamic force coefficients used in the above equations are defined as follows.

$$
\left[\overline{C_{A}}\right]=\frac{C_{A} \rho V S}{2 m} \quad\left[\overline{C_{Y}}\right]=\frac{C_{Y} \rho V S}{2 m} \quad\left[\overline{C_{N}}\right]=\frac{\rho V S C_{N}}{2 m}
$$

The initial conditions must be specified for the above six ODEs in order to perform a 6-DOF simulation. These initial conditions are defined in the global coordinate system at the instance the projectile leaves the tube. As shown in Equation 2-22, the initial velocity of the projectile was determined by knowing the angle at which it exits the barrel relative to the ground, $\theta_{0}$, and the angle at which it departs to the right or left of the barrel, $\psi_{0}$. These angles are also known as the pitch attitude and heading angles, respectively. The sign convention is defined such that $\theta_{0}$ is positive upwards and $\psi_{0}$ is positive to the right looking downrange.

$$
\vec{V}=\left[\begin{array}{c}
u_{0} \\
v_{0} \\
w_{0}
\end{array}\right]=V_{0}\left[\begin{array}{c}
\cos \theta_{0} \cos \psi_{0} \\
\sin \theta_{0} \cos \psi_{0} \\
\sin \psi_{0}
\end{array}\right]
$$

The CFD simulation coupled with the 6-DOF flight dynamics model express the numerical flight test data in the global coordinate system. These parameters include the position, linear and angular velocities, and the three Euler (bank, pitch attitude, heading) angles at the CG of the tube-launched transformable MAV. The simulation begins by initializing the flow domain and specifying the initial flight conditions. The CFD model uses a segregated pressure-based solver to compute the velocity components, mass continuity, and turbulence properties and reiterates the solution until the residuals converge. The turbulence properties include the turbulent kinetic energy and specific dissipation rate. Once the convergence criterion is satisfied, the three aerodynamic forces and three aerodynamic moments are transferred to the 6-DOF model. The translational velocities are computed globally by first solving for the translational and angular accelerations along the body axes, as shown in Equation 2-16 through Equation 2-21, followed by a coordinate transformation to calculate the global translational accelerations. Equation 2-23 provides the transformation matrix, $\overline{\bar{D}}$, used to calculate the global accelerations.

$$
\left\{\begin{array}{l}
V_{1} \\
V_{2} \\
V_{3}
\end{array}\right\}=\overline{\bar{D}}\left\{\begin{array}{l}
u \\
v \\
w
\end{array}\right\}
$$

where,

$$
\overline{\bar{D}}=\left[\begin{array}{ccc}
\cos \theta \cos \psi & \sin \varphi \sin \theta \cos \psi-\cos \varphi \sin \psi & \cos \varphi \cos \theta \cos \psi+\sin \varphi \sin \psi \\
\cos \theta \sin \psi & \sin \varphi \sin \theta \cos \psi+\cos \varphi \cos \psi & \cos \varphi \sin \theta \sin \psi+\sin \varphi \cos \psi \\
-\sin \theta & \sin \varphi \cos \theta & \cos \varphi \cos \theta
\end{array}\right]
$$

The translational accelerations are then numerically integrated twice to determine the global linear velocity components and global position components. The angular accelerations about the body axes are numerically integrated to evaluate the roll, pitch, and yaw rates. These angular rates are then 
numerically integrated to solve for the time rate of change of the three Euler angles using the transformation matrix provided in Equation 2-24. By performing another numerical integration, the updated Euler angles determine the new orientation of the aerodynamic body. The process is repeated upon each successive time step in which the dynamic mesh is updated by performing smoothing and remeshing of unstructured cells. A flowchart is provided below in Figure 2-4 that illustrates the technique for computing the free flight motion parameters using the coupled CFD/6-DOF solver.

$$
\left\{\begin{array}{c}
\dot{\phi} \\
\dot{\theta} \\
\dot{\psi}
\end{array}\right\}=\overline{\bar{A}}^{-1}\left\{\begin{array}{l}
P \\
Q \\
R
\end{array}\right\}
$$

where,

$$
\overline{\bar{A}}^{-1}=\left[\begin{array}{ccc}
1 & \tan \theta \sin \phi & \tan \theta \cos \phi \\
0 & \cos \phi & -\sin \phi \\
0 & \sin \phi / \cos \theta & \cos \phi / \cos \theta
\end{array}\right]
$$

The 6-DOF motion history parameters as well as the aerodynamic forces and moments are computed in the global coordinate system. However, this information is not useful for analyzing the dynamics of the tube-launched transformable MAV. Therefore, this requires the linear and angular velocities to be manually calculated in the body axis system. Equation 2-25 computes the translational velocities along the body axes by taking the inverse of the transformation matrix, $\overline{\bar{D}}$, as provided in Equation 2-23.

$$
\left\{\begin{array}{l}
u \\
v \\
w
\end{array}\right\}=\bar{D}^{-1}\left\{\begin{array}{l}
V_{1} \\
V_{2} \\
V_{3}
\end{array}\right\}
$$

where,

$$
\overline{\bar{D}}^{-1}=\left[\begin{array}{ccc}
\cos \psi \cos \theta & \sin \psi \cos \theta & -\sin \theta \\
-\sin \psi \cos \phi+\cos \psi \sin \theta \sin \phi & \cos \psi \cos \phi+\sin \psi \sin \theta \sin \phi & \cos \theta \sin \phi \\
\sin \psi \sin \phi+\cos \psi \sin \theta \cos \phi & -\cos \psi \sin \phi+\sin \psi \sin \theta \cos \phi & \cos \theta \cos \phi
\end{array}\right]
$$

As shown in Equation 2-26, the body forces are evaluated using the inverse of the transformation matrix as provided above. Once the body forces are determined, the lift and drag can be calculated by applying Equation 2-3 and Equation 2-4.

$$
\left\{\begin{array}{l}
F_{A} \\
F_{Y} \\
F_{N}
\end{array}\right\}=\overline{\bar{D}}^{-1}\left\{\begin{array}{l}
F_{1} \\
F_{2} \\
F_{3}
\end{array}\right\}
$$




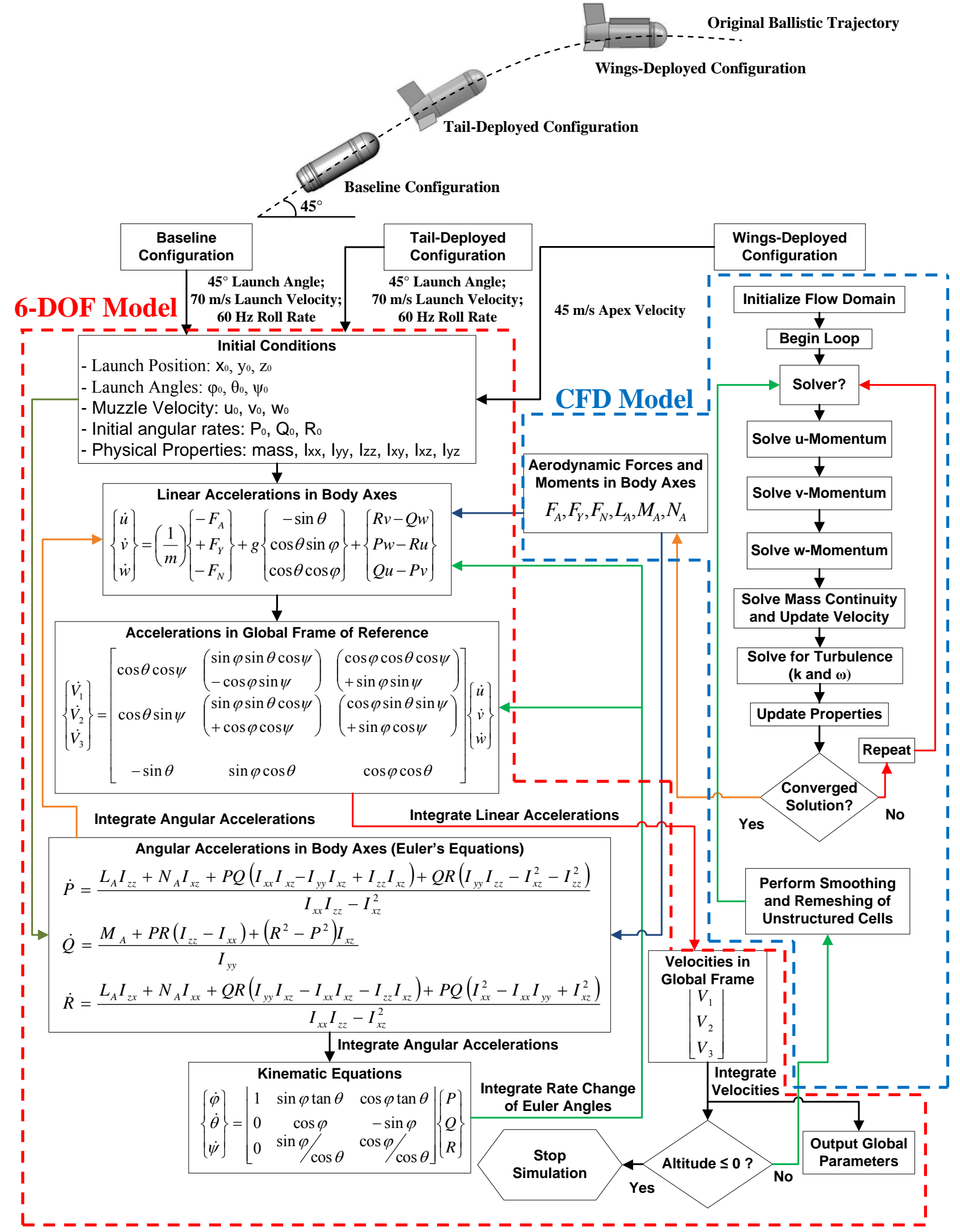

Figure 2-4: Flowchart of CFD Simulation Coupled to 6-DOF Model for Numerical Flight Testing 
Before proceeding further in presenting the numerical flight test results on the tube-launched transformable MAV, a review of some prior research studies utilizing the advanced technique of coupling a CFD simulation with a 6-DOF rigid body flight dynamics model will be discussed. Three different papers will demonstrate that this relatively new tool can provide accurate results of an aerodynamic body's free flight motion. As will be shown, the results of numerical flight testing will be validated using experimental methods.

Because this research involves the numerical flight testing of a $40 \mathrm{~mm}$ round in the subsonic flow regime, a prior study by Sahu (2006) that utilizes the coupled CFD/6-DOF simulation tool for the purpose of evaluating the dynamic stability of a $40 \mathrm{~mm}$ subsonic projectile will be discussed. Figure 2-5 presents the geometry of the $40 \mathrm{~mm}$ round as well as the structured mesh consisting of approximately 4 million nodes. The simulation was performed using a hybrid RANS/LES model provided in CFD++ for approximately 0.5 second at an initial speed of Mach 0.39 and a spin rate of $434 \mathrm{~Hz}$. This required between 32 and 64 processors and thousands of central processing unit time. For comparison, spark range tests were conducted to photograph the position and angular orientation of the projectile at fixed intervals. The data was then transferred to the Aeroballistics Research Facility Data Analysis System (ARFDAS) to generate the curves representing the pitching and yawing motion. Figure 2-6 provides a computational and experimental comparison of the pitching and yawing motion that clearly shows the $40 \mathrm{~mm}$ round is dynamically stable.

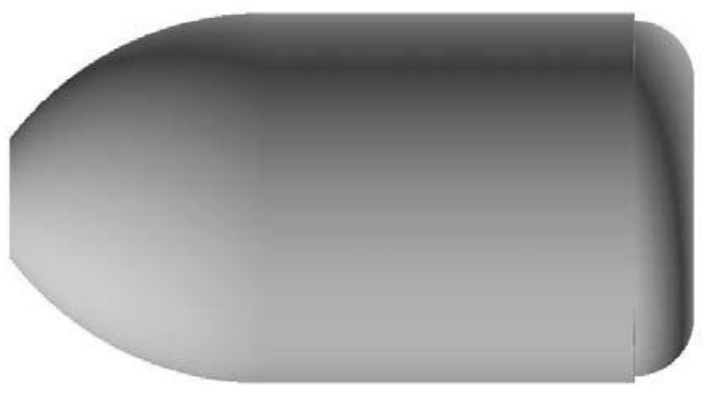

(a)

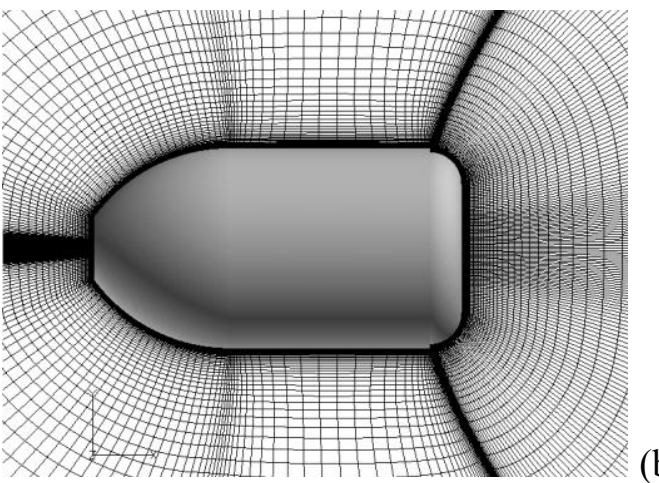

(b)

Figure 2-5: 40 mm Subsonic Projectile Round (a) Geometry and (b) Computational Grid
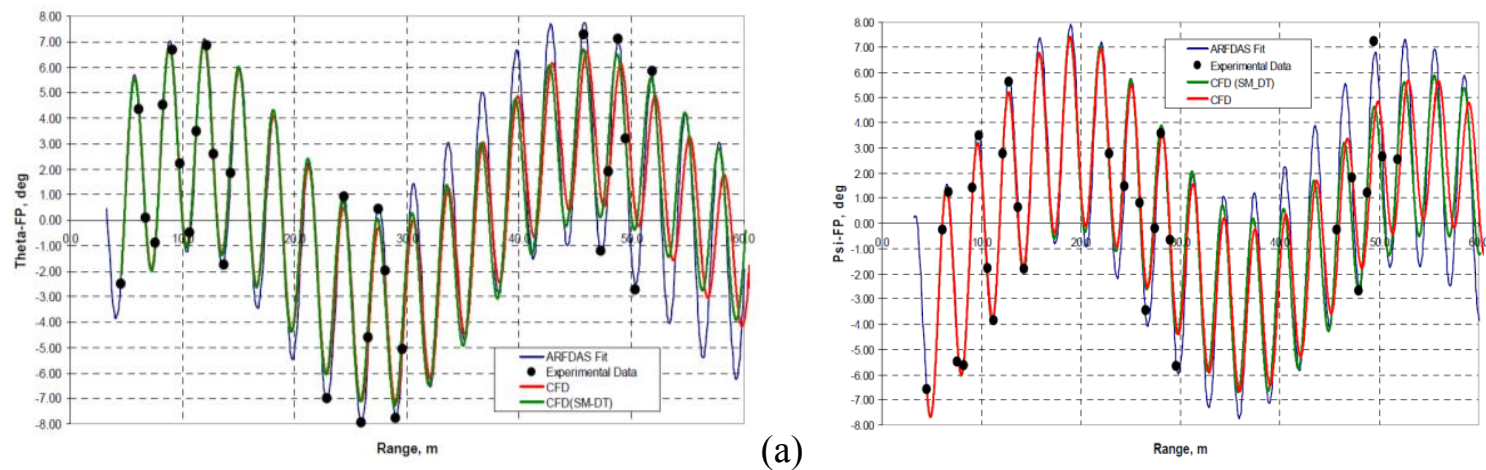

(b)

Figure 2-6: 40 mm Subsonic Projectile Round (a) Pitching and (b) Yawing Motion

Another paper that is relevant to the current research presents a free flight motion study by Sahu (2005) on a $25 \mathrm{~mm}$ ojive-cylinder-finned configuration to predict its unsteady free flight aerodynamic behavior. The total length of the projectile body is $121 \mathrm{~mm}$ with a total of four fins having a $2.5^{\circ}$ boat-tail and a fin thickness of approximately $10 \mathrm{~mm}$. Figure 2-7 provides a geometric description of the finned projectile in addition to the computational $\mathrm{C}$-grid that encompasses the entire body. Using CFD++, two 
separate free flight test simulations are conducted with the first simulation being initialized at the muzzle and the second simulation being initialized at the first station away from the muzzle where the experimental data is measured. The initial conditions for the first and second flight tests are provided in Table 2-1.

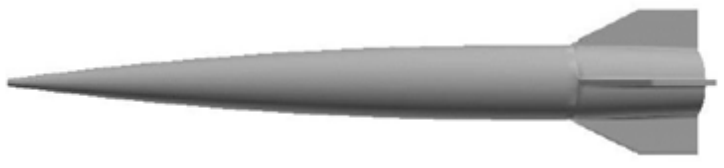

(a)

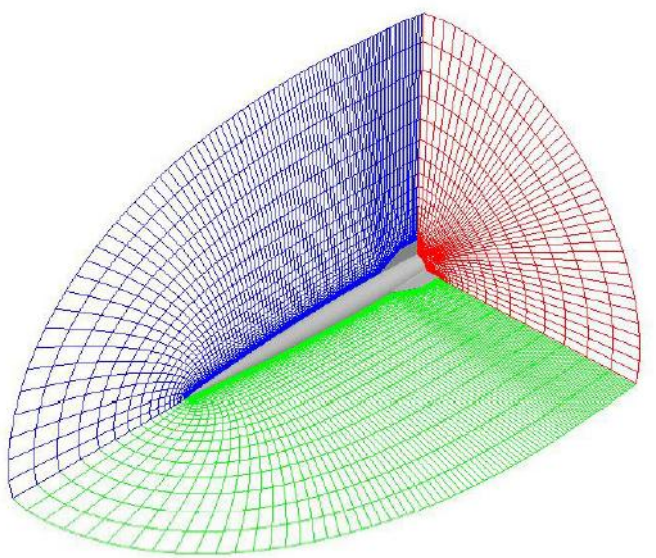

(b)

Figure 2-7: 25 mm Ojive-cylinder-finned Projectile (a) Geometry and (b) Computational Grid

Table 2-1: Initial Parameters for Two Flight Test Simulations on 25 mm Projectile

\begin{tabular}{|c|c|c|c|}
\hline Parameters & Muzzle & First Station & Units \\
\hline Velocity & 1,037 & 1,034 & $\mathrm{~m} / \mathrm{s}$ \\
\hline Roll Rate & 2,800 & 2,500 & $\mathrm{rad} / \mathrm{s}$ \\
\hline Angle of Attack & 0.5 & 4.9 & $\mathrm{deg}$ \\
\hline
\end{tabular}

Sahu (2005) shows that the two free flight test simulations and experimental measurements from spark range testing agree quite well with one another. Figure 2-8 shows the amplitudes dampening in the pitch attitude and yaw angles, which indicates that the finned projectile becomes more stable as it travels downrange. The time histories of the pitch attitude and yaw angles are plotted against each other that illustrate the path of the projectile's nose viewed from its base looking downrange. Figure 2-9 provides a good agreement between the computational data shown on the left and experimental data on the right. Similar to the previous study, the spark range test measurements were transferred to the ARFDAS software to generate the curves representing the pitching and yawing motion. Additionally, the ARFDAS software was used to plot the experimentally measured total angle of attack and aerodynamic forces (axial, normal, side) along the body axes, as provided in Figure 2-10. This shows that the experimental data compares well with the computational analysis. Similar to the dynamics of the pitch attitude and yaw angles, there is considerable dampening in the amplitudes of the aerodynamic force data as the projectile travels further downrange. This dynamic behavior is directly related to the dampening in the total angle of attack. 

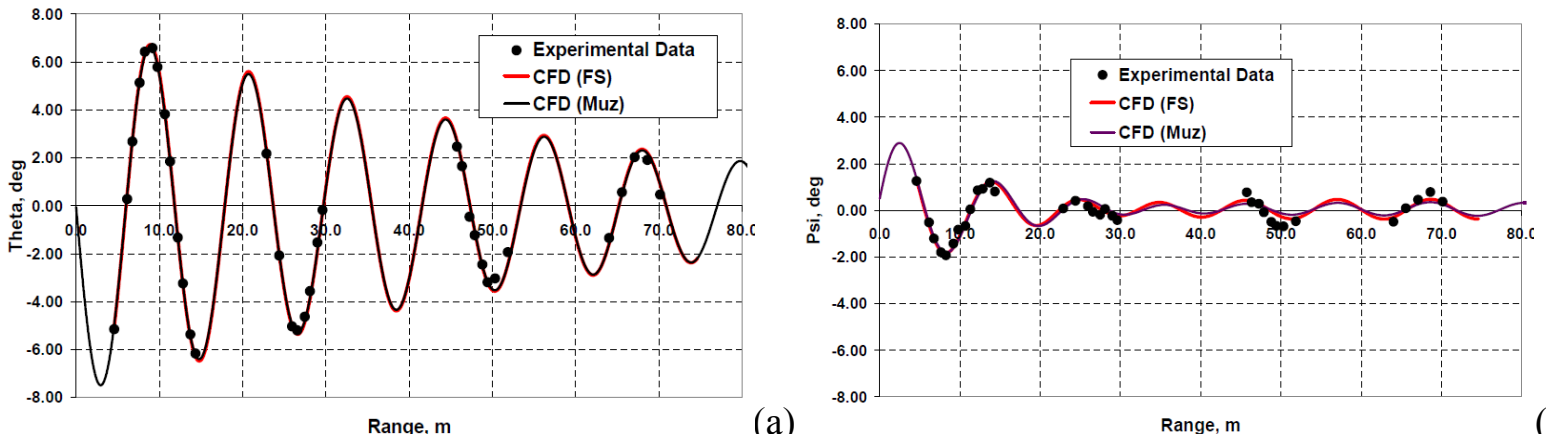

(a)

Range, $m$

(b)

Figure 2-8: Numerical and Experimental Data for (a) Euler Pitch Attitude Angle and (b) Euler Yaw Angle
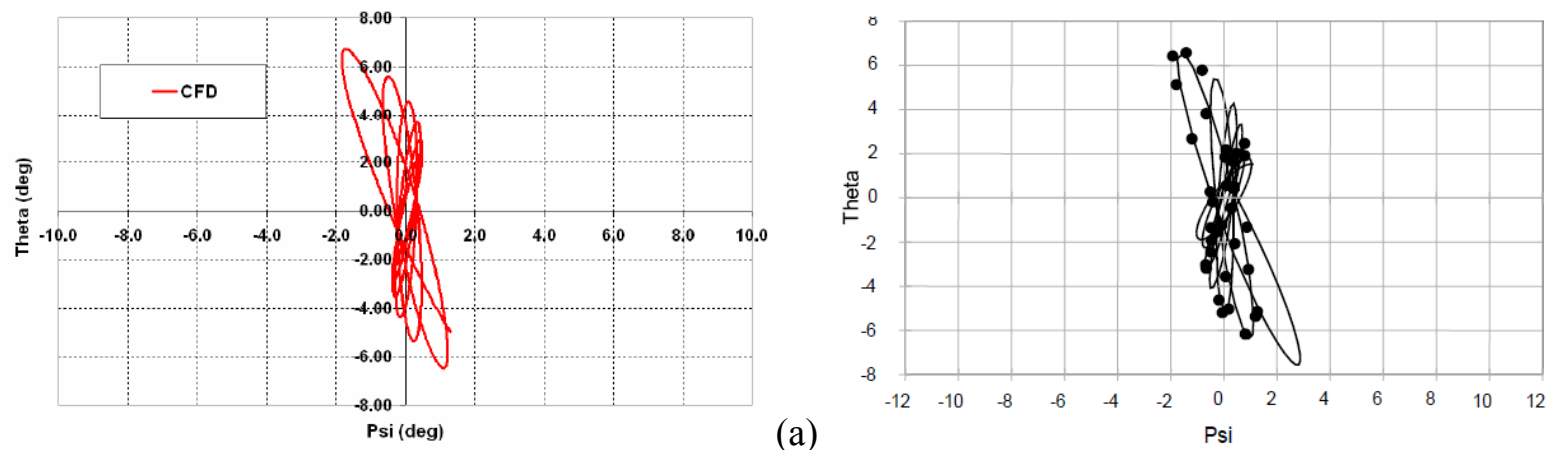

(b)

Figure 2-9: Motion History of Projectile Viewed from the Rear Looking Downrange using (a) Computational Approach and (b) Experimental Approach
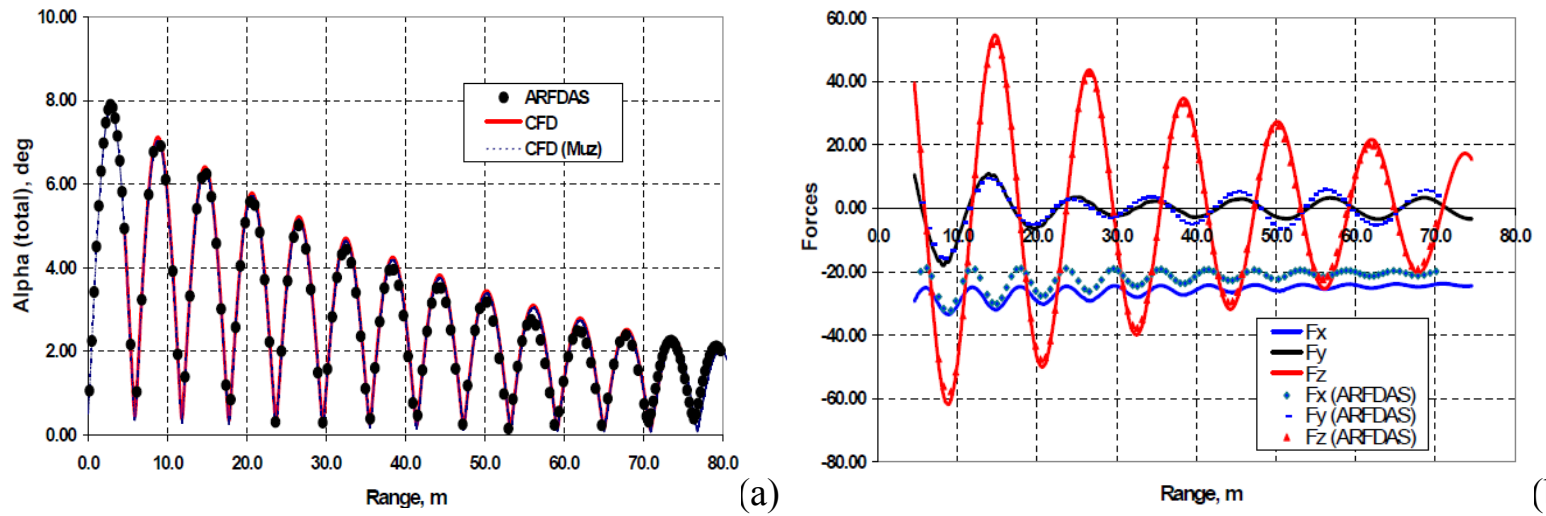

(b)

Figure 2-10: Numerical and Experimental Data for (a) Total Angle of Attack and (b) Aerodynamic Forces

The second paper presents a flight dynamics simulation by Murman, et. al. (2003) in which a Navy GBU-31 Joint Direct Attack Munition (JDAM) store is separated from a pylon below the left wing of an F/A-18C aircraft. This simulation uses a 6-DOF model coupled with an inviscid CFD solver. The motivation of this research is to predict the unsteady aerodynamic behavior immediately after being released from the pylon that includes analyzing the relative displacements and angular positions in roll, 
pitch, and yaw as well as the angular rates. Figure 2-11 shows an image of a GBU-31 mounted to the pylon below the left wing of the F/A-18C aircraft. Additionally, Figure 2-9 provides a cut plane through the Cartesian volume mesh that illustrates greater cell density around the wings as well as around the GBU-31.
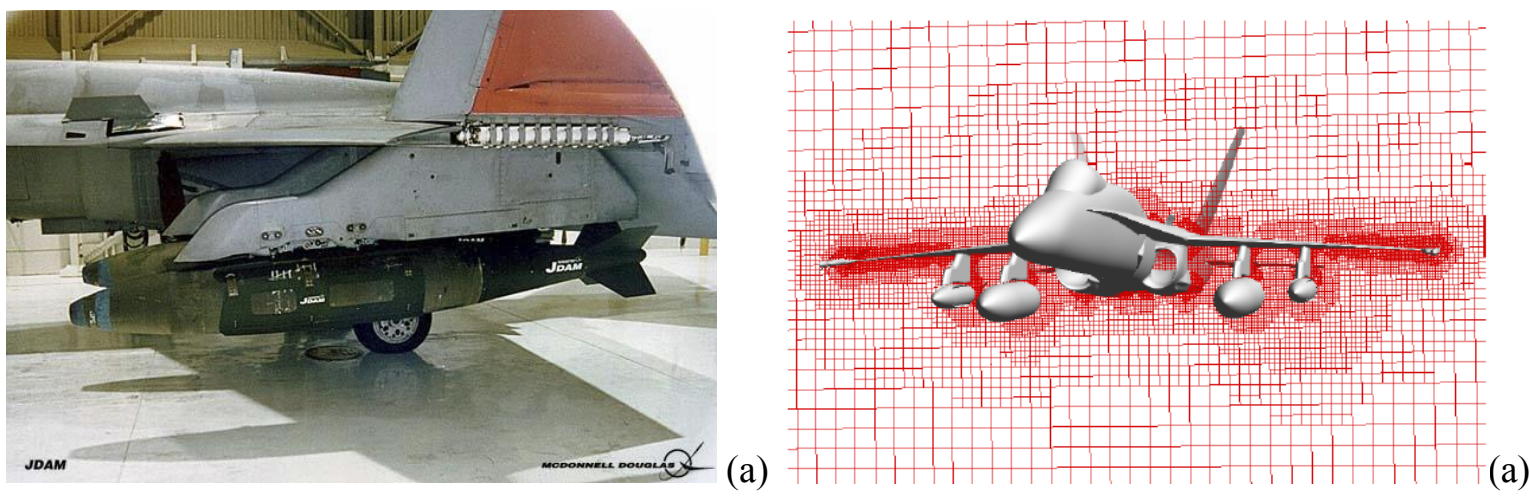

Figure 2-11: F/A-18C Showing (a) GBU-31 Mounted to Left Wing Pylon and (b) Cut Plane through Mesh

Two different simulations are performed that include sequential-static and time-dependent simulations. The disparity between the two methods is that the CFD/6-DOF flight dynamics solvers are uncoupled in the sequential-static simulations, whereas the solvers are fully coupled in the timedependent simulation. As explained by Murman et. al. (2003), both methods are expected to provide similar results since the inertia of the GBU-31 is quite large thereby eliminating large unsteady effects close to the separation point. In addition to these flight test simulations, measured data was obtained from two different experimental techniques and compared with the computational results. These experimental techniques include obtaining measurements derived from in-flight photography and flight test telemetry.

The flight conditions for the simulations are at a Mach number of 1.055, an angle of attack of $-0.65^{\circ}$, and an angle of sideslip of $44^{\circ}$. As provided in Figure 2-12, the two simulations show good agreement in the relative distances and angular positions prior to $0.125 \mathrm{sec}$. In comparison to the experimental data, the accuracy in the pitch and yaw rates decrease at $0.125 \mathrm{sec}$ and $0.2 \mathrm{sec}$, respectively. At these specific instances in time, the angular rates are maximum and the acceleration changes signs indicating that these are regions where unsteady effects may be considerably larger. As shown in Figure 2-13, the two simulations agree well with one another for the pitch and yaw rates except for the dynamic response at the maximum pitch and yaw rates. Figure 2-14 illustrates the pressure contours plotted on the surface of the F/A-18C aircraft in which an overlay of multiple sequential-static simulations show the flight trajectory of the JDAM store upon separation from the aircraft. Similar research work was performed by Panagiotopoulos and Kyparissis (1990) using a coupled CFD/6-DOF simulation tool to predict the trajectory of a store separating from the pylon of an aircraft in the transonic flow regime. Their results of this relatively new simulation tool showed good agreement with wind tunnel test measurements. 

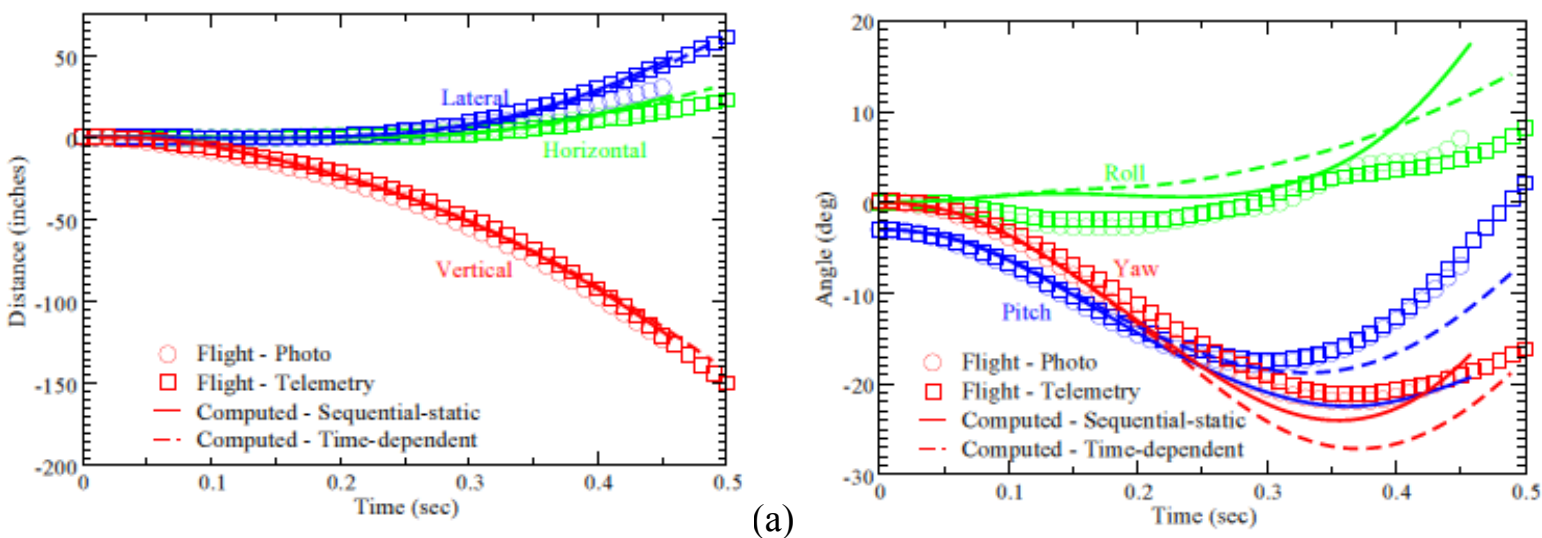

Figure 2-12: Results of (a) Distance Traveled and (b) Angular Position after JDAM Store Separation

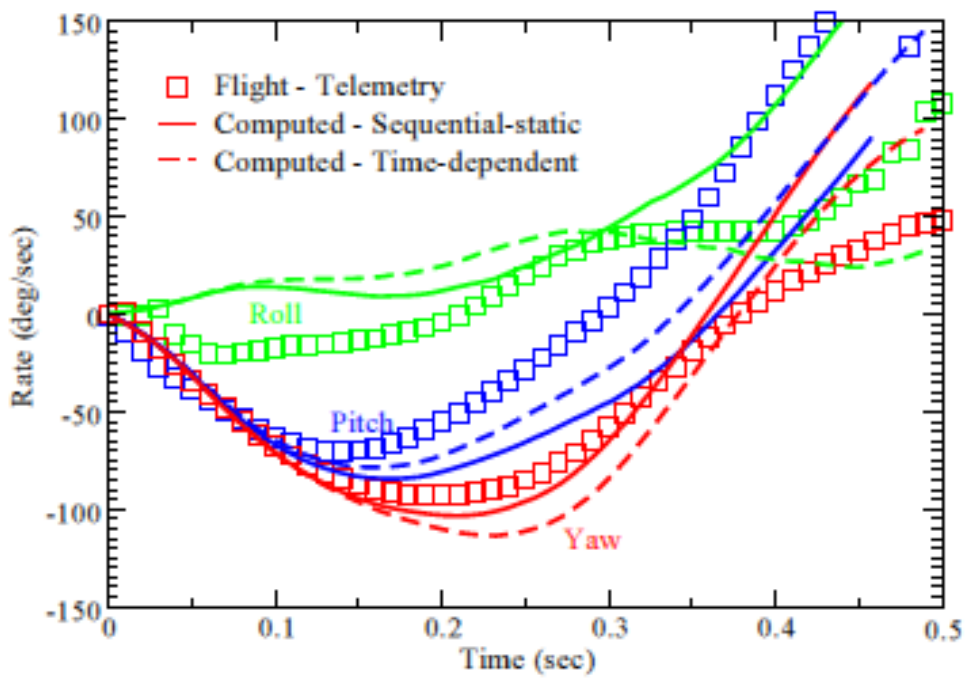

Figure 2-13: Results of Angular Rates about Body Axes after JDAM Store Separation

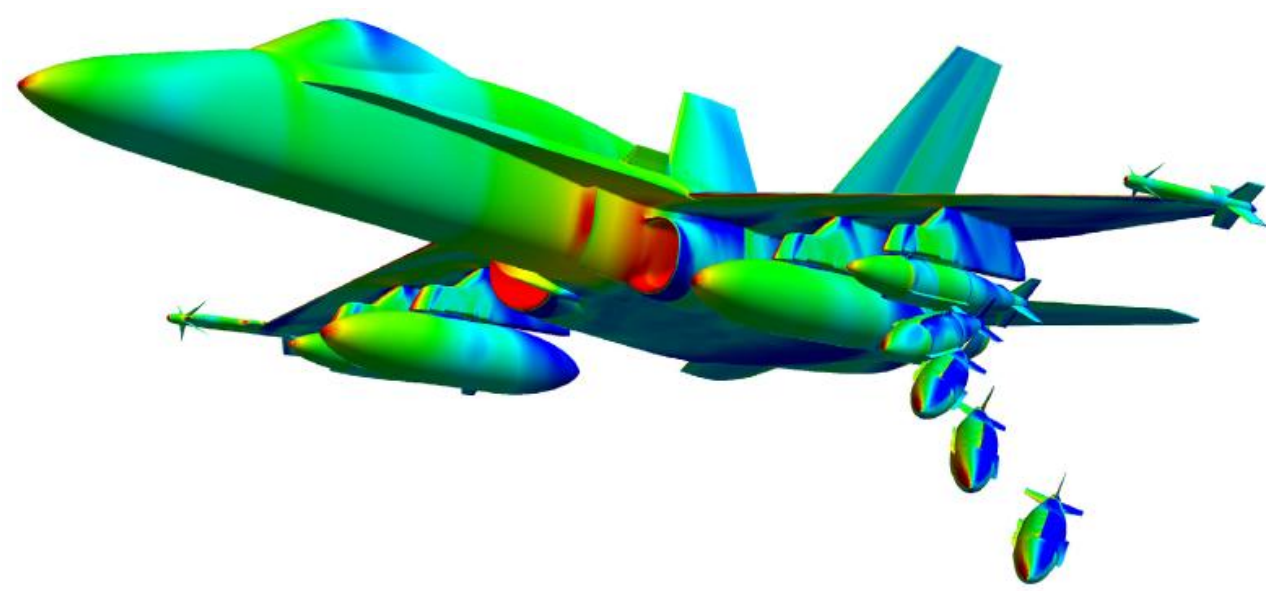

Figure 2-14: Predicted Flight Trajectory of JDAM Store with Plotting of Surface Pressure Contours 


\subsection{Modeling of Flow Physics}

Because these numerical flight test simulations involve more than just a standard 6-DOF tool in that the CFD solver is tightly coupled to the 6-DOF flight dynamics model, this requires the physics of the airflow to be realistically modeled around the tube-launched transformable MAV. The importance of selecting a realistic model is so that the CFD solver can accurately compute the aerodynamic forces and moments before passing them to the 6-DOF equations of motion.

In Hamburg (2010), prior CFD studies were performed on the original baseline design having a cylindrical length of $80 \mathrm{~mm}$. Since the design speed places the MAV in the low Reynolds number regime having an approximate Reynolds number of 191,000 at launch, CFD studies were initially performed using the laminar model. However, the this model showed problems in obtaining a converged solution using the steady-state solver because of the presence of a large recirculation region at the base of the body, as shown in Figure 2-15. These recirculation regions were generated by initially activating turbulence in the flow before switching to the laminar model. Therefore, the standard k- $\omega$ turbulence model was selected over the laminar model as it provided a more accurate steady-state solution; however, the turbulence model was more computationally expensive. Additionally, the k- $\omega$ based Shear Stress Transport (SST) turbulence model was executed and the results of aerodynamic drag were compared to the standard k- $\omega$ model.
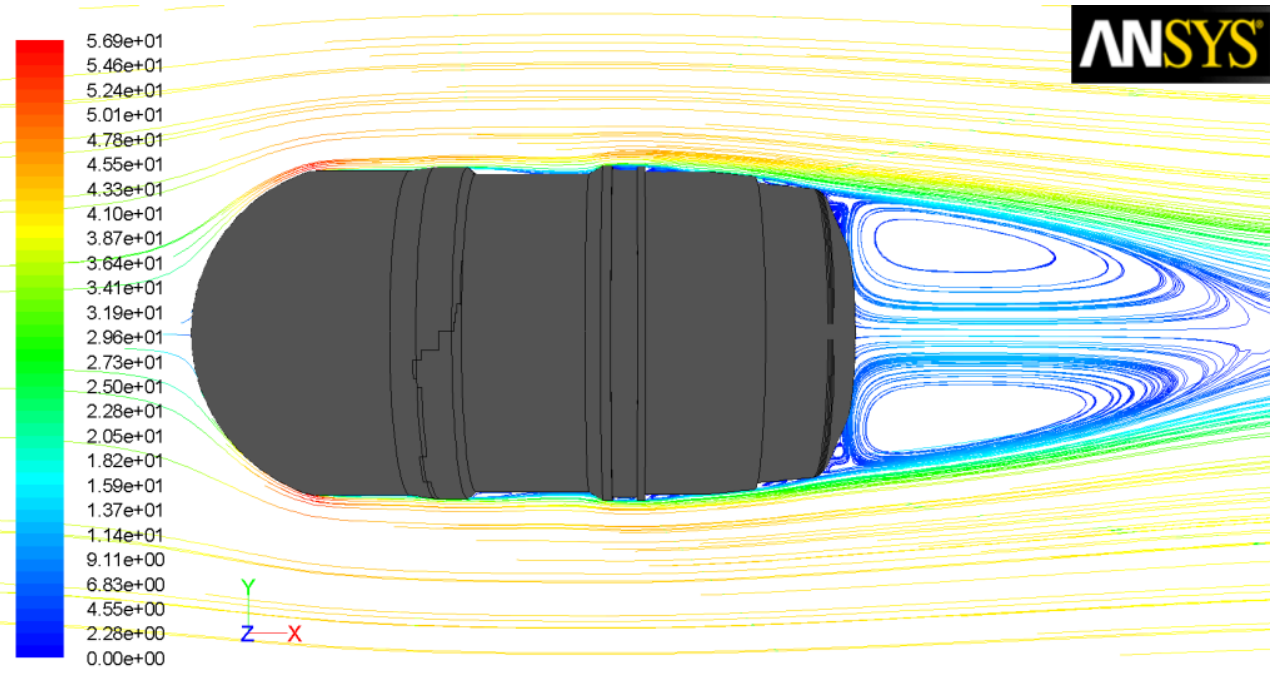

Figure 2-15: Pathlines Colored by Velocity Magnitude Showing Recirculation Region Aft of Original Baseline Design using Standard k- $\omega$ Model

In comparison to the experimentally determined drag coefficient of 0.133 , the calculated drag on the baseline was under predicted by approximately 7\% using the standard k- $\omega$ model, whereas the k- $\omega$ SST model over predicted the drag by $8 \%$. In an engineering point of view, the SST k- $\omega$ model was a better choice for conducting an aerodynamics analysis on the baseline as this model was a more conservative approach. For consistency, the SST k- $\omega$ turbulence model was selected for the fin-stabilized and fully deployed MAV configurations. It is important to note that for these two turbulence models to make accurate predictions, the average value for the dimensionless distance from the surface of the aerodynamic body, $y^{+}$, is suggested to be no greater than 1, as indicated by Lun (2011), and is provided in Equation 2-27.

$$
y^{+}=\frac{u^{*} y}{v}<1
$$




\subsection{Ballistics}

One of the requirements for this study was to analyze the aerodynamic behavior of a projectile once it had been launched from the tube system. As a result, this required a basic understanding of ballistics. There are four major categories to consider in the study of ballistics that include interior, intermediate, exterior, and terminal ballistics. Because this research was concerned with simulating a projectile upon leaving the barrel of the tube for a short time duration and that the pressure from the explosive gases were no longer transferring kinetic energy to the projectile, the study of exterior ballistics would only be of interest.

There are many factors to consider in the study of exterior ballistics, but only a few will be taken into account in this study. The simulated dynamics of the baseline round will be affected by the launch angle and factors associated with spinning the aerodynamic body. Such factors include gyroscopic, Magnus, and Coriolis effects. While Coriolis drift occurs as a result of the Earth's rotation in long range ballistics, it will have a negligible effect due to the short time duration.

When designing a spin-stabilized symmetric projectile, the engineer must evaluate both the gyroscopic and dynamic stabilities in order to make accurate predictions of its pitching and yawing motion. This requires the pitching moment coefficient derivative, $C_{M_{\alpha}}$, to be known. For a spin-stabilized projectile, it inherently has a positive pitching moment due to the placement of the CG aft of the center of pressure (CP), causing the projectile to eventually become unstable. However, the combination of projectile spin about its longitudinal axis and the pitching moment result in a gyroscopic precession that eventually damps out if the projectile is both gyroscopically and dynamically stable. Figure 2-16 shows the $40 \mathrm{~mm}$ baseline round with the polar and transverse moments of inertia at the CG location. The placement of the CG was selected based on the traditional CFD analyses conducted on the fully deployed MAV configurations, which will be discussed later. It should be noted that for symmetric projectiles, the two transverse moments of inertia, $I_{y y}$ and $I_{z z}$, are equal.

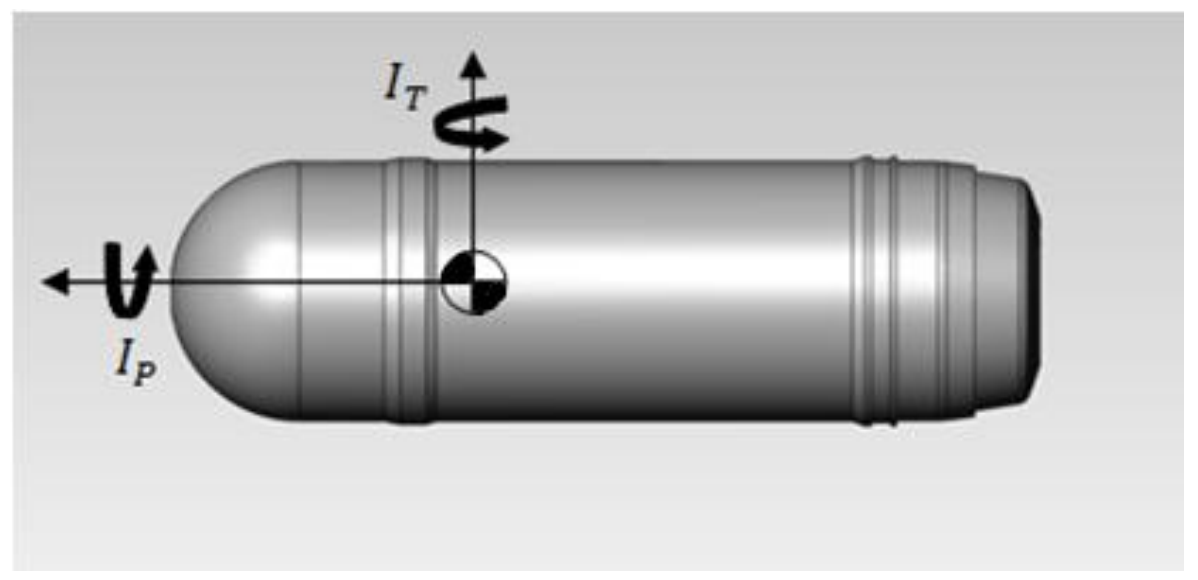

Figure 2-16: $40 \mathrm{~mm}$ Baseline Showing CG Location and Polar and Transverse Moments of Inertia.

The classical criterion for gyroscopic stability provided in Equation 2-28, shows that the gyroscopic stability factor, $S_{g}$, must be greater than 1 to satisfy the criterion.

$$
S_{g}=\frac{I_{P}^{2} P^{2}}{2 \rho I_{T} S d V^{2} C_{m_{\alpha}}}>1
$$

The dynamic stability factor, $S_{d}$, is also defined as provided in Equation 2-29. Unlike the gyroscopic stability factor, the dynamic stability factor can be difficult to accurately calculate because it 
requires additional stability derivatives to be known, such as the Magnus moment coefficient derivative, $C_{m_{p \alpha}}$, and the pitch damping moment coefficient, $\left(C_{m_{q}}+C_{m_{\dot{\alpha}}}\right)$.

$$
S_{d}=\frac{2\left(C_{L_{\alpha}}+\frac{m d^{2}}{I_{P}} C_{m_{p \alpha}}\right)}{C_{L_{\alpha}}-C_{D}-\frac{m d^{2}}{I_{T}}\left(C_{m_{q}}+C_{m_{\dot{\alpha}}}\right)}
$$

Despirito and Heavey (2006) presented a study on a spin-stabilized $25 \mathrm{~mm}$ M910 TPDS-T subcaliber training projectile to investigate its aerodynamic coefficients. This research compared results of various CFD turbulence models with wind tunnel testing and the commercially available Projectile Design Analysis System (PRODAS) software at a range of Mach numbers and angles of attack. According to their study, the CFD results showed that the Magnus moment coefficient derivative was unreliable in the subsonic and transonic flow regimes. Figure 2-17 provides this data for the computational and experimental approaches at $3^{\circ}$ AOA. The hybrid RANS/LES detached-eddy simulation (DES) showed a better comparison with the experimental data, but this required significantly more computational time due to the simulation being time-dependent.

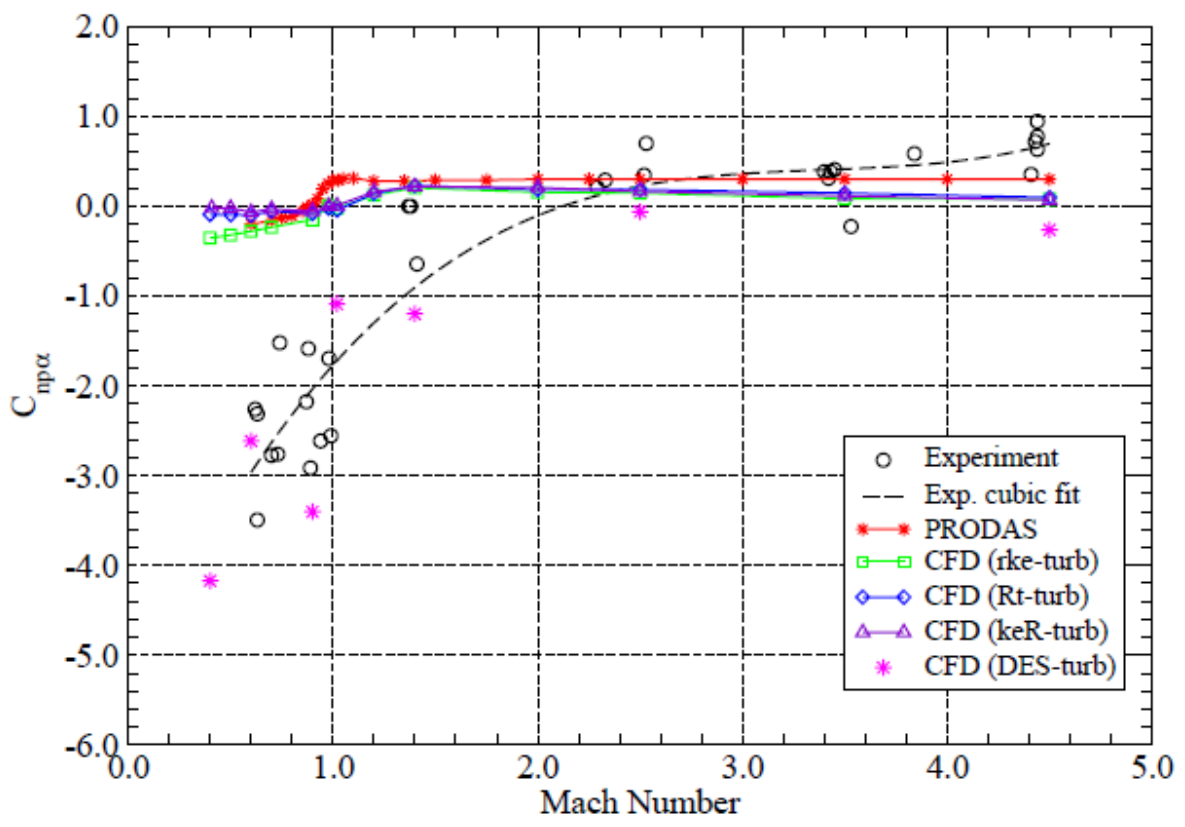

Figure 2-17: Computational and Experimental Methods Showing Inconsistent Data for Magnus Moment Coefficient in Sub-sonic Flow Regime (DeSpirito and Heavey, 2006).

It was noted in their research using the DES model that the Magnus moment coefficient in the subsonic flow regime produced large oscillatory motion most likely due to the oscillatory wake flow, as shown in Figure 2-18. Figure 2-19 presents erratic data for the Magnus moment coefficient occurring at a velocity in the subsonic flow regime. This proved to be difficult in determining a mean value for the force and moment time histories as well. As mentioned in the research by Despirito and Heavey (2006), the experimental error could be as large as the error in the DES simulation, as shown in Figure 2-19. 


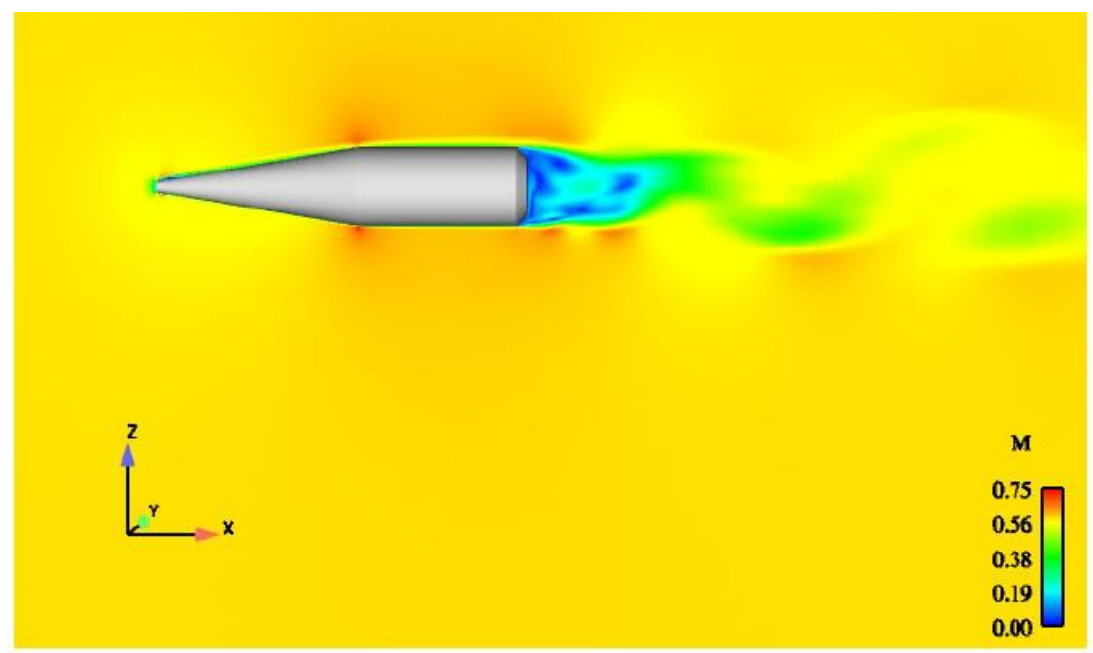

Figure 2-18: Mach Number Contours on Pitch Plane at Mach 0.3 using Time-accurate DES Model (DeSpirito and Heavey, 2006).

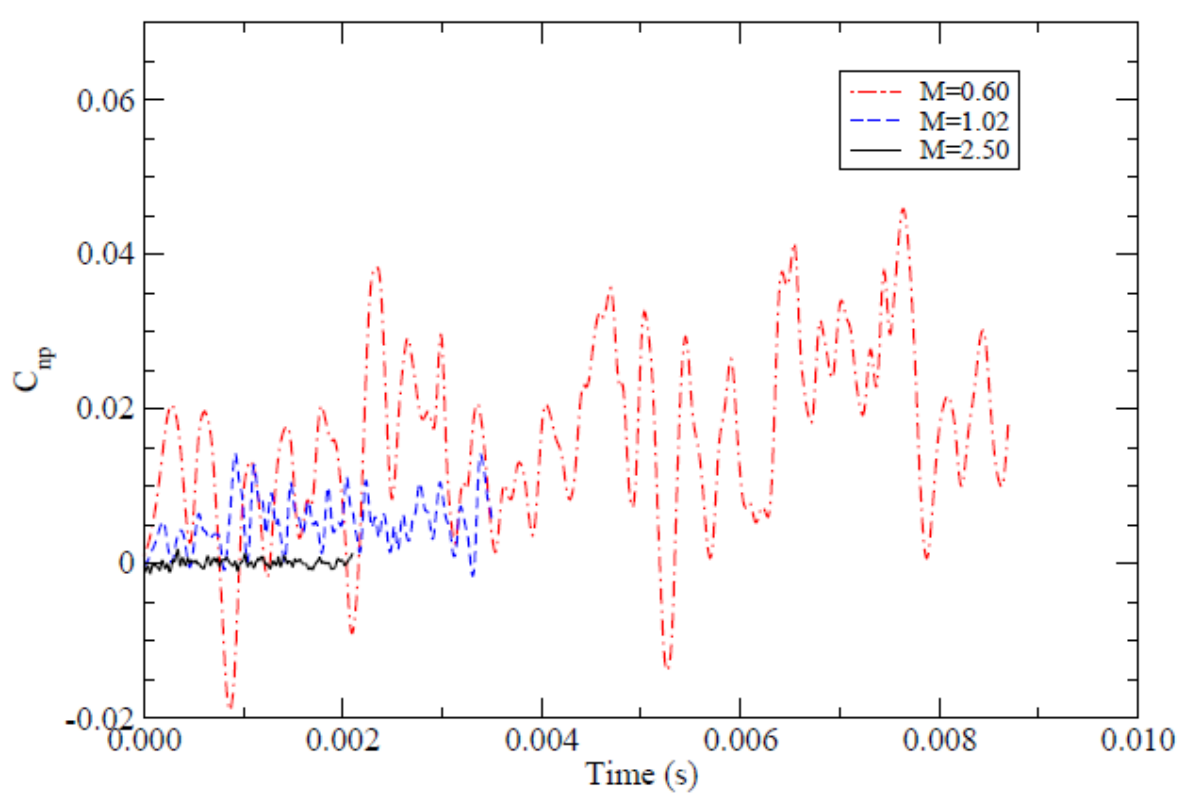

Figure 2-19: Magnus Moment Coefficient at Subsonic, Transonic, and Supersonic Flow Regimes for $3^{\circ}$ AOA (DeSpirito and Heavey, 2006).

In Valyou and Marzocca (2011), wind tunnel testing was conducted for a $40 \mathrm{~mm}$ round design to measure the time required for the projectile to de-spin. Figure 2-20 shows the projectile mounted to a sting, which allowed the projectile to rotate using a bearing system. This experiment showed that the projectile required approximately $9 \mathrm{sec}$ to de-spin for an initial spin rate of $60 \mathrm{~Hz}$ or $3600 \mathrm{RPM}$, as provided in Figure 2-21.

Similar to the CFD study performed by DeSpirito and Heavey (2006) on the $25 \mathrm{~mm}$ sub-caliber training projectile, the experimentally measured force and moment time histories were difficult to accurately determine. However, in contrast to the numerical errors in the study by DeSpirito and Heavey, the inconsistencies in the force and moment time histories were due to vibration-induced noise. The fluctuating force data provided in Figure 2-22 is the combined side and Magnus forces for the spinning 
projectile at an angle of attack of $11^{\circ}$. Also provided in Figure 2-22 is the fluctuating moment data that is a combination of the yaw and Magnus moments.

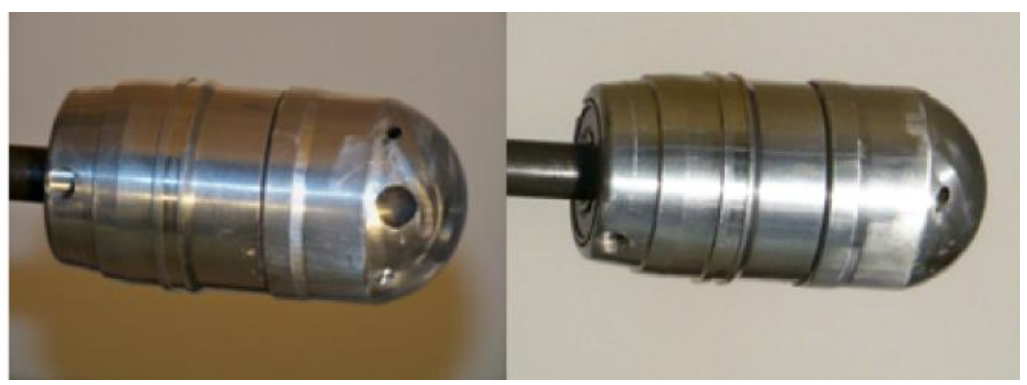

Figure 2-20: $40 \mathrm{~mm}$ Round Design Attached to Sting (Valyou and Marzocca, 2011).

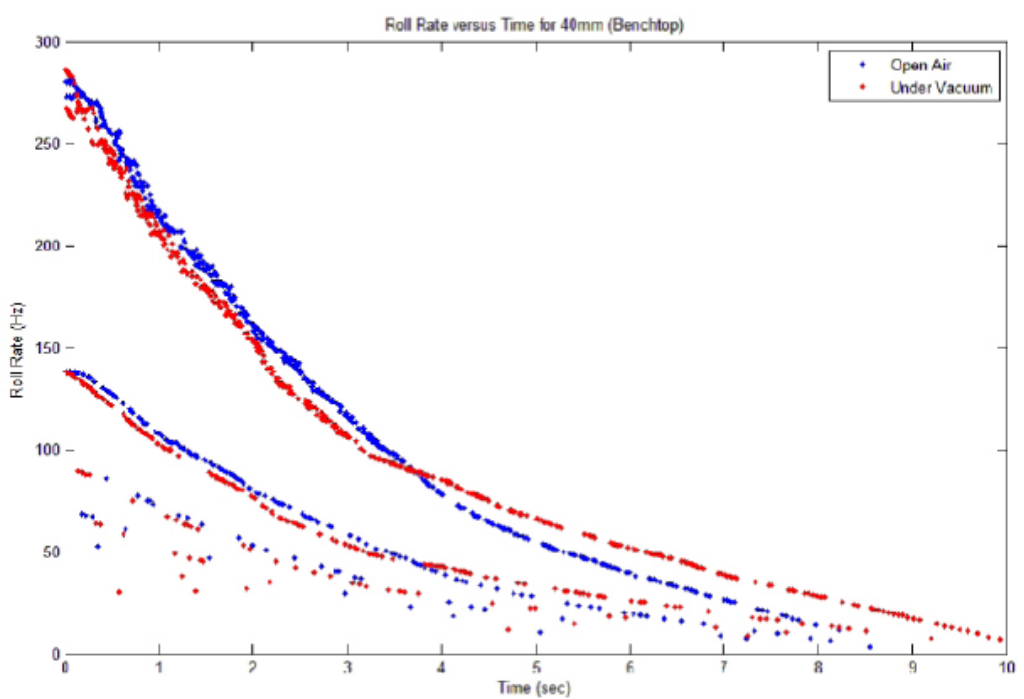

Figure 2-21: Experimental Results of De-spin for $40 \mathrm{~mm}$ Projectile (Valyou and Marzocca, 2011).
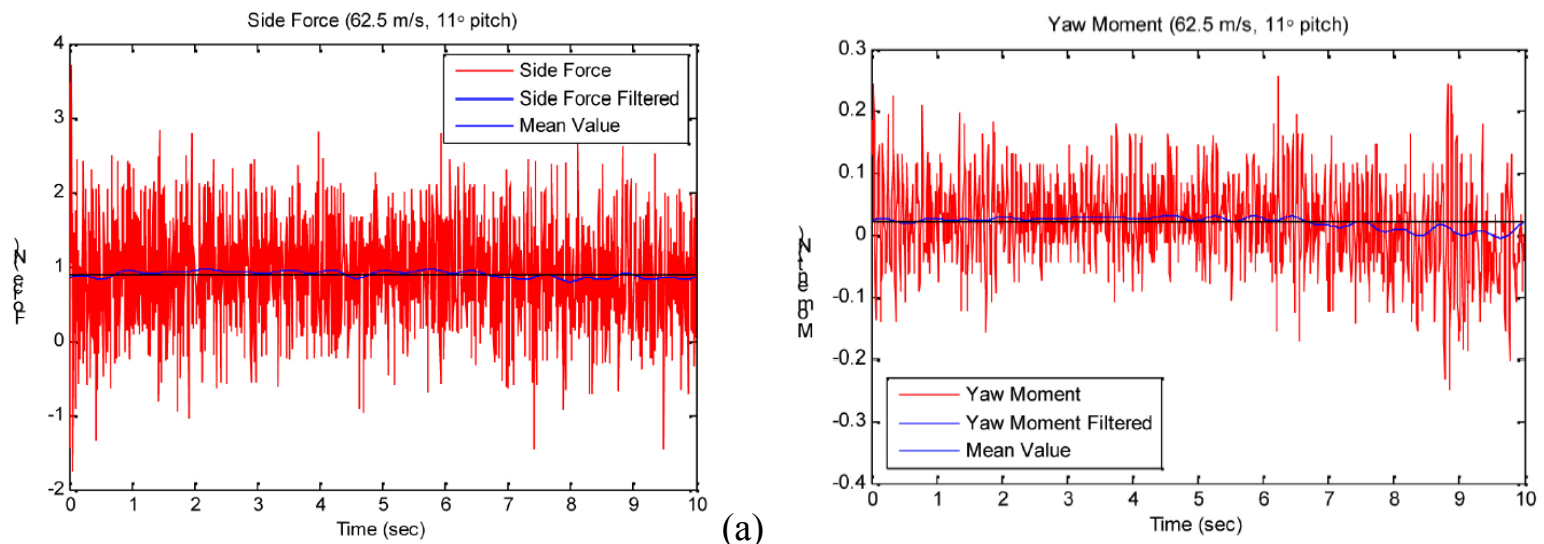

(b)

Figure 2-22: Time Histories of (a) Composite Force and (b) Composite Moment for Spinning $40 \mathrm{~mm}$ Round at Free Stream Velocity of $62.5 \mathrm{~m} / \mathrm{s}$ and $11^{\circ} \mathrm{AOA}$ (Valyou and Marzocca, 2011).

McCoy (1999) provides the detailed criteria for both stable and unstable projectiles. The region for dynamic stability can be described using Equation 2-30 in which both the gyroscopic and dynamic 
stability factors can be plotted against each other. Figure 2-23 shows the regions of dynamic stability and dynamic instability.

$$
S_{g}>\frac{1}{S_{d}\left(2-S_{d}\right)}
$$

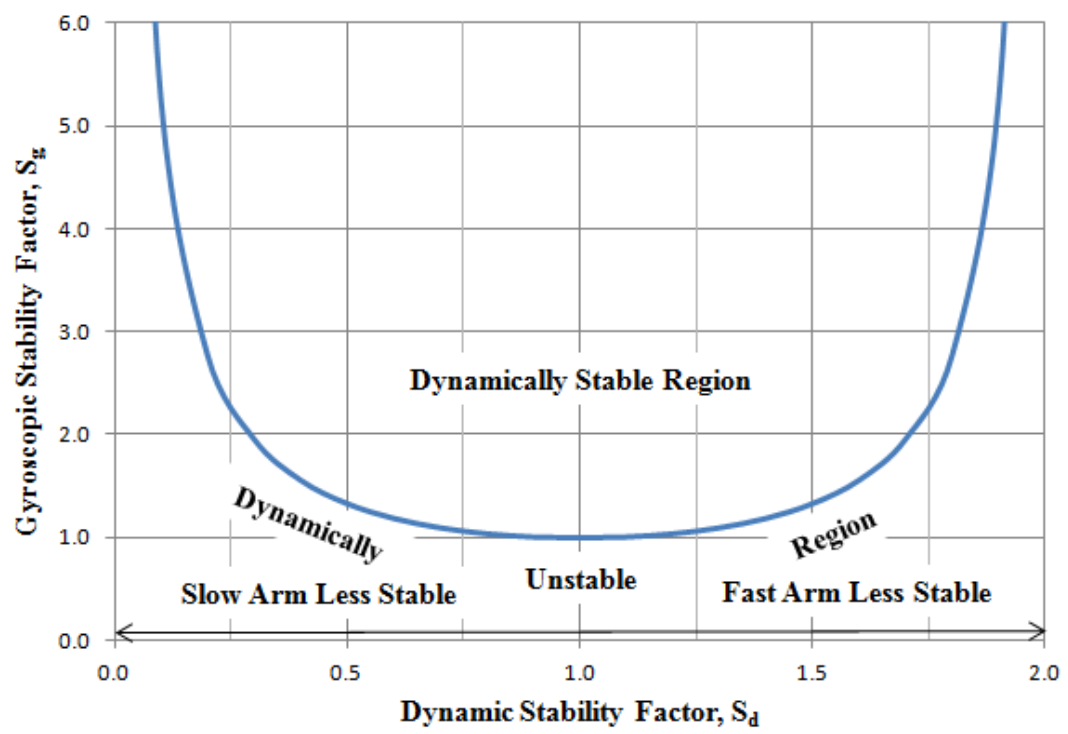

Figure 2-23: Regions of Dynamic Stability and Dynamic Instability for Spinning Projectiles (Reproduced from McCoy, 1999).

The above figure illustrates that even if the projectile is gyroscopically stable, it is not guaranteed to be dynamically stable. A dynamic stability factor of less than one corresponds to a less stable slow arm in the lateral motion whereas a dynamic stability factor greater than one corresponds to a less stable fast arm. The slow and fast arms are also known as precession and nutation, respectively.

Another method to determine if the spin-stabilized projectile is dynamically stable is to observe its pitching and yawing motion. This can be accomplished by performing a CFD analysis coupled with 6-DOF flight prediction to plot the numerical results for angle of attack, $\alpha$, against the angle of sideslip, $\beta$. If the projectile's angle of attack and angle of sideslip continue to increase in magnitude without any appearance of dampening, the projectile is guaranteed to be dynamically unstable even though it may be gyroscopically stable. However, if the projectile's angle of attack and angle of sideslip are bounded by an upper and lower limit, it is said to have a limit-cycle yawing motion.

Lyon (1997) performed a study on a $40 \mathrm{~mm}$ nonlethal cartridge to examine the gyroscopic and dynamic stability of the projectile. The project was carried out using the commercially available PRODAS software to simulate the 6-DOF motion of the $40 \mathrm{~mm}$ round. As opposed to the work performed in this research, the PRODAS software predicts the flight dynamics using a standard 6-DOF solver. The numerical results were then validated by test firing the rounds using a M203 grenade launcher. By performing these test launches, the engineers were able to visually inspect the conical motion in the projectile as it traveled downrange. This data showed the projectile having a poor flight performance in that it flew with a high-limit-cycle yaw of approximately 15 degrees, which was directly related to having a gyroscopic stability factor slightly greater than 1 . Figure $2-24$ provides the simulated results for the projectile's pitching and yawing motion. 


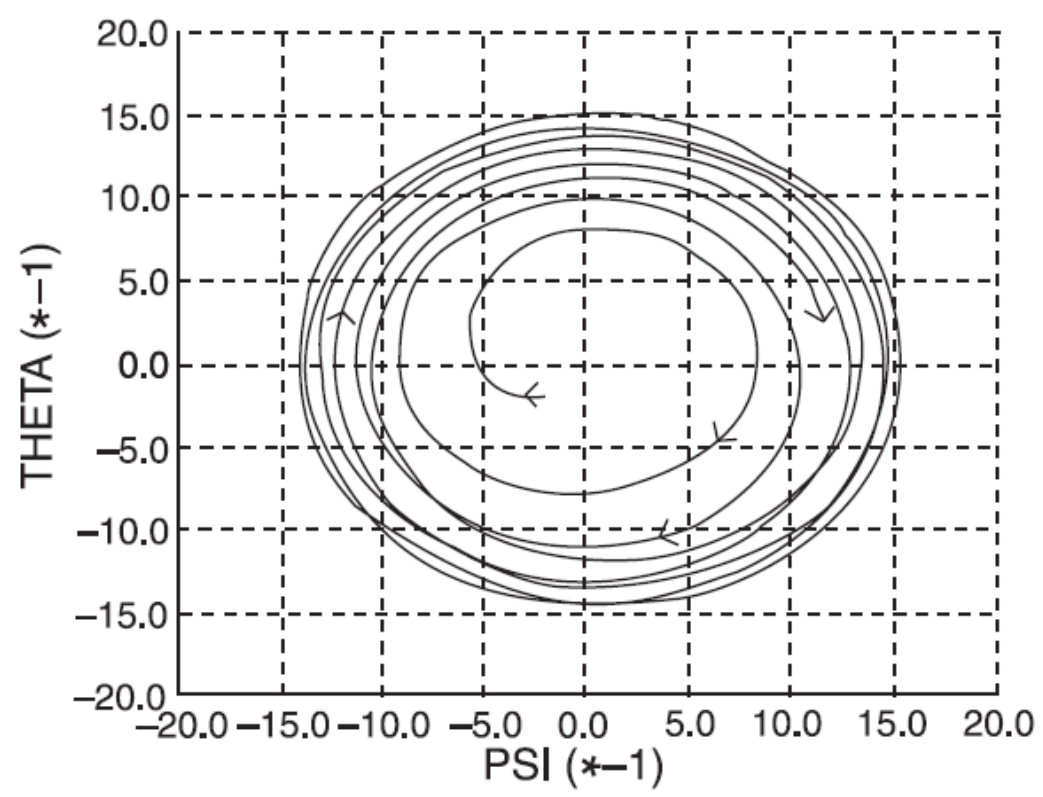

Figure 2-24: Pitching and Yawing Motion of $40 \mathrm{~mm}$ Nonlethal Cartridge (Lyon, 1997).

\subsection{Spin Decay of Fin Stabilized Projectiles}

A major requirement for the tube-launched transformable MAV was to rapidly reduce its spin rate before deploying its wings at the apex of the original ballistic trajectory. The rapid decrease in the spin rate was essential to the design of the transformable MAV in order to avoid undesirable aerodynamic effects in the MAV's longitudinal and later-directional dynamics at the instance of deployment of the wings. The technique for reducing the projectile's spin rate was accomplished by deploying its tail immediately after being launched from the tube system. The tail would not only act as a spin decay enhancement mechanism, but it would also provide a significant improvement to the dynamic stability of the baseline configuration.

Hamburg (2010) performed a simple air trajectory analysis of the $40 \mathrm{~mm}$ round, which required the projectile's mass and drag coefficient to be known. This analysis showed that for a muzzle velocity of $70 \mathrm{~m} / \mathrm{s}$ and launch angle of $45^{\circ}$, the $40 \mathrm{~mm}$ round reached the apex of its ballistic trajectory in approximately $5 \mathrm{sec}$. As presented previously, experimental work performed by Valyou and Marzocca (2011) demonstrated that the time for the $40 \mathrm{~mm}$ round to de-spin given an initial roll rate of $60 \mathrm{~Hz}$ required approximately $9 \mathrm{sec}$, as provided in Figure 2-21. According to this data, the projectile's spin rate at 5 seconds after launch would be roughly $20 \mathrm{~Hz}$ or 1,200 RPM. Clearly, the baseline would not have despun by the time it reached the apex of its ballistic trajectory. Therefore, these two analyses strengthened the concept of implementing a design mechanism to deploy the tail immediately after being launched from the tube to rapidly reduce the projectile's spin rate before deploying the wings at the apex of the original ballistic trajectory.

Since a numerical flight test utilizing the coupled CFD and 6-DOF analysis tools would be performed on the finned projectile to evaluate its spin decay rate and time required to de-spin, an analytical solution to this problem was performed as well for comparison. Two analytical solutions provided by Toledo (2009) were used to compute the spin damping moment coefficient and roll rate. The first analytical solution assumed an elliptical lift distribution on the tail fins, whereas the second analytical solution assumed a triangular lift distribution. Both solutions required the normal force coefficient to be evaluated at the root of the fin, $C_{N_{\alpha_{\text {fin root }}}}$, which involved the chord length and span-wise length of the 
fin geometry to be known. It was assumed that the normal force would be greatest at the root of the tail fin. Once $C_{N_{\alpha_{\text {fin root }}}}$ was calculated in Equation 2-31 assuming an elliptical lift distribution, the spin damping moment coefficient could be determined using Equation 2-32. The negative sign is used to indicate that the spin damping moment is acting against the direction of the projectile's rotation.

$$
\begin{aligned}
& C_{N_{\alpha_{\text {fin root }}}}=\frac{4 C_{N_{\alpha_{\text {fin }}}}}{\pi a c} \\
& C_{l_{p}}=\frac{-2 c C_{N_{\alpha_{\text {fin root }}}}}{a d^{2}}\left(\frac{\pi a^{4}}{16}+\frac{\pi r^{2} a^{2}}{4}+\frac{2 r a^{3}}{3}\right)
\end{aligned}
$$

Similarly, the above equations can be modified for a triangular lift distribution using Equation 2-33 and Equation 2-34 to compute $C_{N_{\alpha_{\text {fin root }}}}$ and the spin damping moment coefficient, respectively.

$$
\begin{gathered}
C_{N_{\alpha_{\text {fin root }}}}=\frac{2 C_{N_{\alpha_{\text {fin }}}}}{a c} \\
C_{l_{p}}=\frac{-c C_{N_{\alpha_{\text {fin root }}}}\left(a^{2} r_{0}^{2}+\frac{2}{3} a^{3} r_{0}+\frac{a^{4}}{6}\right)}{a d^{2}}
\end{gathered}
$$

Using the spin damping moment coefficient from Equation 2-32 and 2-34, the final spin rate could be evaluated, as provided in Equation 2-35. It was necessary to account for the number of fins, $\mathcal{N}$, attached to the body of the projectile since each fin contributed to reducing the spin rate.

$$
P_{f}=P_{i} e^{\mathcal{N} A C_{l p}\left(t_{f}-t_{i}\right)}
$$

where,

$$
A=\frac{q S d^{2}}{2 I_{p} V}
$$

\subsection{Thesis Outline}

The structuring of this thesis was organized in such a way that it explains the step-by-step procedures for conducting a simulation undergoing free body motion using ANSYS Fluent. Before making any attempts to generate a mesh, it was necessary to determine the feasibility in meshing such a complex geometry. The baseline did not require any modifications; however, the tail-deployed and fully deployed MAV configurations did require simplifications to the wing and tail designs. This was primarily due to the geometric complexities in the deployment mechanisms of the control surfaces.

Once the model simplifications were finalized, the geometries were imported into ANSYS ICEM CFD for meshing. Because the baseline was the most simplistic of the three models and did not require 
any modifications to the geometry, a mesh for the baseline configuration was generated first. Following a successful mesh for this model, meshes were then generated for the tail-deployed and wings-deployed configurations. Chapter 3 will further discuss and provide details for creating the various meshes. Before running traditional CFD cases to obtain angle of attack sweeps for the aerodynamic coefficients, such as lift and drag, a mesh independent study was required. That is, multiple meshes of varying resolution were generated for each configuration to show that the results became independent of mesh size. The grid independence studies will be presented in Chapter 4 along with the results of the aerodynamic coefficients. Chapter 5 provides the initialization requirements to conduct a free body motion analysis. This will discuss the implementation of the dynamic mesh feature provided in ANSYS Fluent as well as the 6-DOF solver utilized for free body motion. In Chapter 6, an analysis of the longitudinal and lateraldirectional stability characteristics will be presented. Additionally, the aerodynamic force characteristics will be discussed as well. Finally, Chapter 7 will summarize the results obtained from numerical flight testing and will provide recommendations on how to proceed with development of the $40 \mathrm{~mm} \mathrm{HP}$. As shown below in Table 2-2, an outline of the research conducted in this thesis is organized as follows.

Table 2-2: Thesis Outline Summary

1.) Mesh Generation for Baseline Configuration

2.) Mesh Generation for Tail-deployed Configuration

3.) Mesh Generation for Wings-deployed Configuration

4.) Mesh Independent Studies for Fixed Body CFD Analyses

5.) Fixed Body CFD Analysis for Baseline Configuration

6.) Fixed Body CFD Analysis for Tail-deployed Configuration

7.) Fixed Body CFD Analysis for Wings-deployed Configuration

8.) Initialization Requirements for Numerical Flight Testing

9.) Numerical Flight Testing for Baseline Configuration

10.) Numerical Flight Testing for Tail-deployed Configuration

11.) Numerical Flight Testing for Wings-deployed Configuration

12.) Summary and Recommendations 


\section{Chapter 3 Mesh Generation Details}

The simulations performed in this study utilized ANSYS ICEM CFD for generating the meshes of the representative geometries. A mesh was first generated for the baseline configuration before proceeding on to generate meshes for the tail-deployed and fully deployed MAV configurations. This was due to the fact that the baseline was more simplistic and did not require any modifications to the geometry. The mesh quality was an important factor in grid generation in which the quality ranged between a magnitude of 0 and 1 in which ANSYS Fluent required a mesh quality to be no less than 0.1 .

\subsection{Baseline Configuration}

A mesh for the baseline was generated prior to performing any work related to this research, as discussed in Hamburg (2010). However, the mesh was created for the original $40 \mathrm{~mm}$ baseline design consisting of a total length of $80 \mathrm{~mm}$ and would require another mesh of the extended round having a total length of $133 \mathrm{~mm}$. Before attempting to generate a mesh for the $40 \mathrm{~mm}$ round, additional geometric surfaces had to be created. Two of these surfaces included a velocity inlet and pressure outlet for conducting traditional CFD simulations in which the airflow traveled relative to a fixed body. The shape of the combined inlet and outlet surfaces resembled a gumdrop shape. The idea behind this geometry was such that the velocity inlet boundary would not interfere with the direction of the free stream flow within the range of angles of attack.

In addition to these surfaces, a geometric body needed to be created around the projectile's surrounding area that was large enough to capture the turbulent flow using an entirely structured mesh. Therefore, a spherical body was selected. On the outside surface of this body, an unstructured mesh was generated that had considerably less cell density than the structured mesh. This low cell density was acceptable since the physics of the airflow was less important as the fluid moved further away from the projectile body. Because this mesh consists of both structured and unstructured cells, this type of mesh is known as a hybrid mesh. At the surface of the sphere, the structured and unstructured cells are not conformal, which requires an interface boundary condition to compute the flux across the non-conformal interface. Figure 3-1 shows the design of the flow domain in which the $40 \mathrm{~mm}$ round is contained within the sphere along with the inlet and outlet boundaries colored in green and red, respectively.

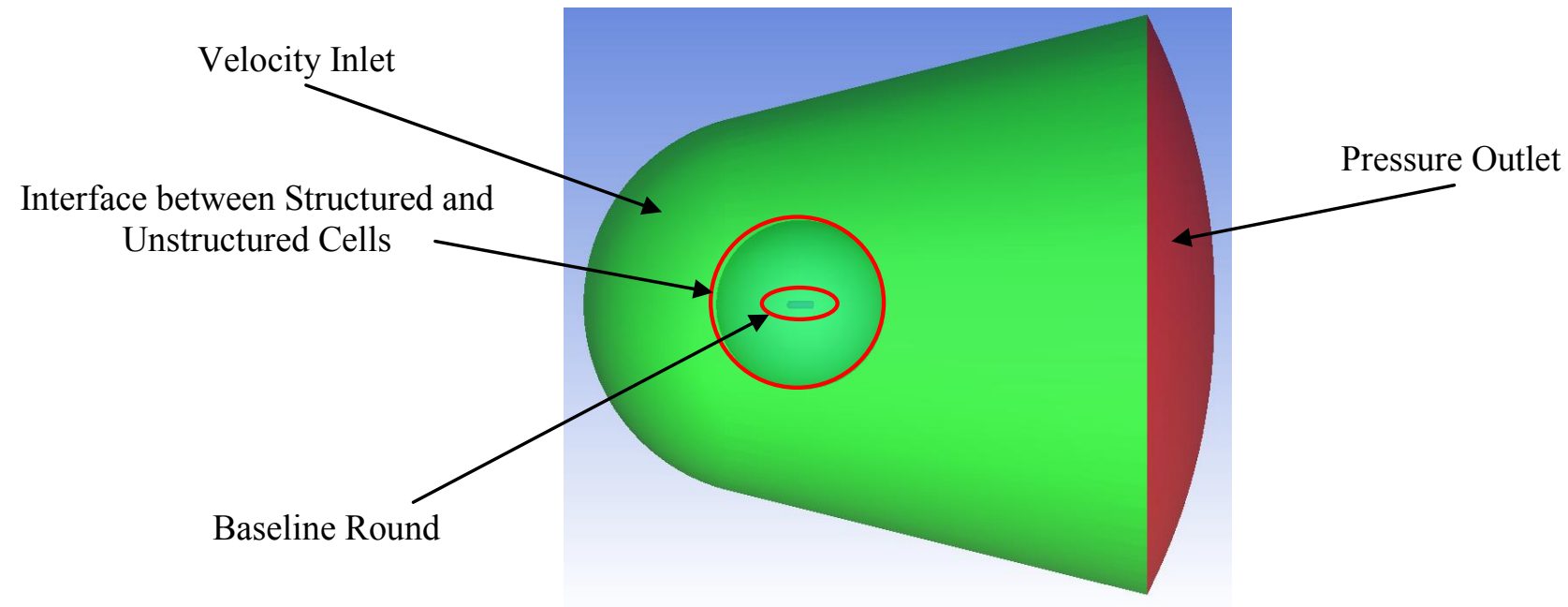

Figure 3-1: Geometric Design of Flow Domain in ICEM CFD for conducting Fixed Body CFD Simulations of $40 \mathrm{~mm}$ Baseline. 
The action of generating a hybrid mesh was considered for three reasons. First and foremost, a structured mesh was desirable on the inner portion of the fluid domain surrounding the projectile because it could provide more control over the cell density than that of unstructured meshes. This was advantageous since turbulence models, such as the k- $\omega$ SST model, require the average $y^{+}$value to be less than 1 in order to make accurate predictions of the flow characteristics. Structured meshes do, however, require considerable amount of time and effort to generate cells with acceptable quality. Secondly, it was not necessary for the solution to accurately predict the flow characteristics far downstream of the projectile since most of the turbulence effects dissipate due to the action of fluid molecular viscosity. Therefore, this justified using an unstructured mesh with relatively large cell density at the non-conformal interface and decreasing cell density moving outward to the inlet and outlet boundaries. Lastly, an unstructured mesh was considered as a result of the numerical flight testing that would later be performed in this research study. That is, the capability in ANSYS Fluent was limited to re-meshing of unstructured cells. Therefore, the structured mesh that consisted of the sphere and its interior components would act as a rigid body. This will be explained in more detail later in this research.

In ICEM CFD, the technique for creating structured meshes using blocks was to use a top-down approach. This was accomplished by beginning with a large block that encompassed the spherical body. The block was then sectioned into smaller blocks by creating a combination of splits and O-grids, to form the shape of the fluid around the baseline configuration. Three meshes of varying resolution were generated and the average number of nodes and cells were approximately 1,075,000 and 1,316,000, respectively. For each of the mesh sizes, the average $y^{+}$value was less than 1 . This was accomplished by first generating the low resolution mesh and performing test simulations to compute the average $y^{+}$value. Once an acceptable $y^{+}$was achieved, the first cell height off the wall was noted and specified in the medium and fine resolution meshes. This technique guaranteed that the desired $y^{+}$value was achieved. Figure 3-2 shows a medium resolution mesh of the $40 \mathrm{~mm}$ round that clearly defines its geometry, such as the hemispherical nose and obturator seats. From this view, it is difficult to see how the mesh wraps around the rear casing of the $40 \mathrm{~mm}$. Therefore, as provided in Figure 3-3, the aft end of the projectile is magnified to clearly show the mesh. Figure 3-4 presents a cross-section of the entire fluid domain consisting of the structured and unstructured cells. The structured mesh represents the inner fluid domain whereas the unstructured mesh represents the outer fluid domain.

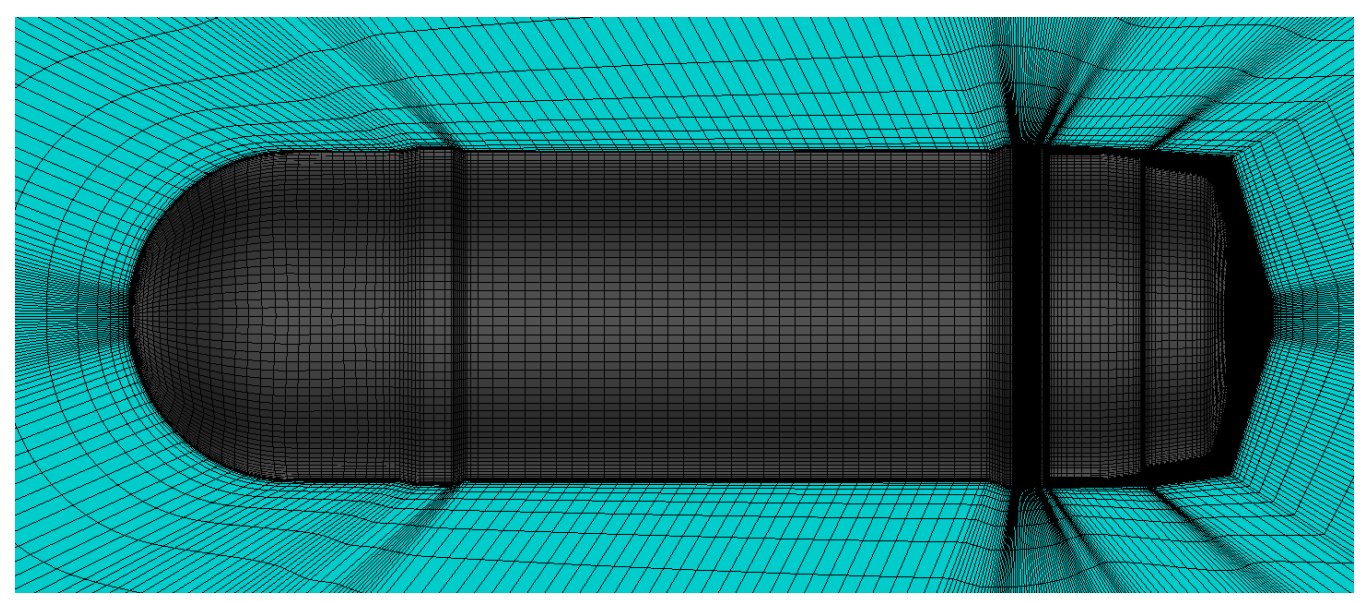

Figure 3-2: Medium Resolution Mesh of $40 \mathrm{~mm}$ Baseline and Inner Fluid Domain. 


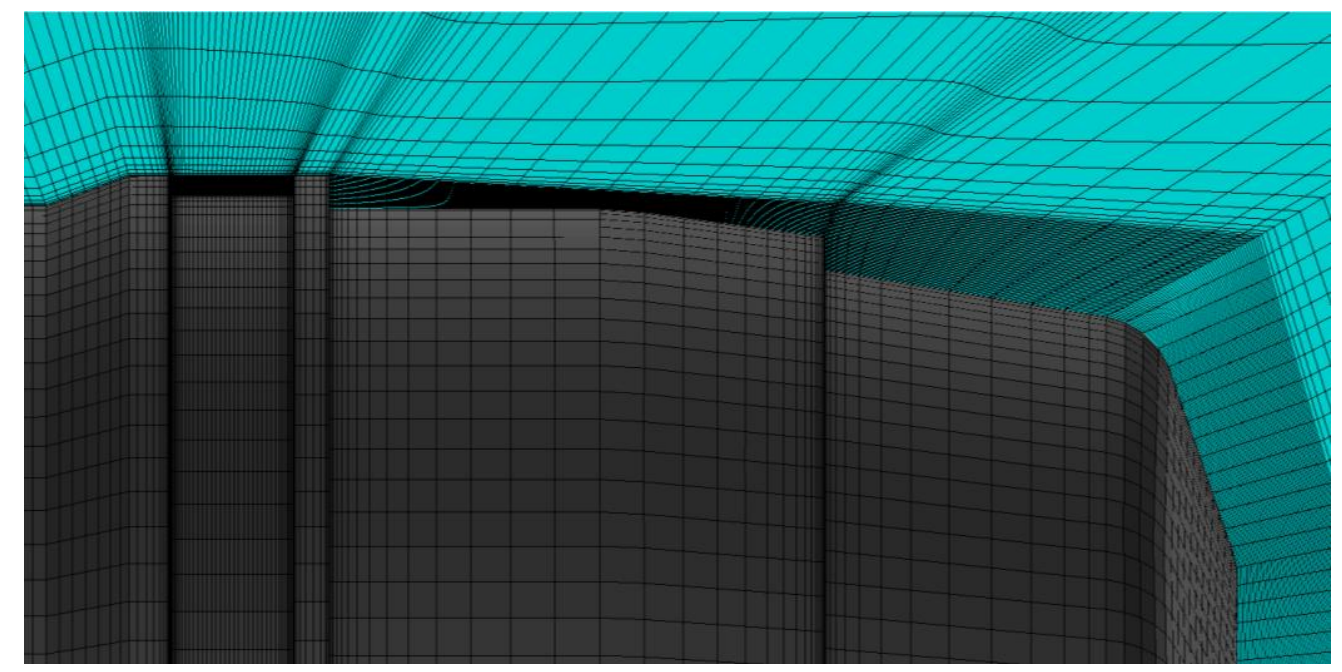

Figure 3-3: Mesh Wrapped around $40 \mathrm{~mm}$ Obturator Seats and Rear Casing.

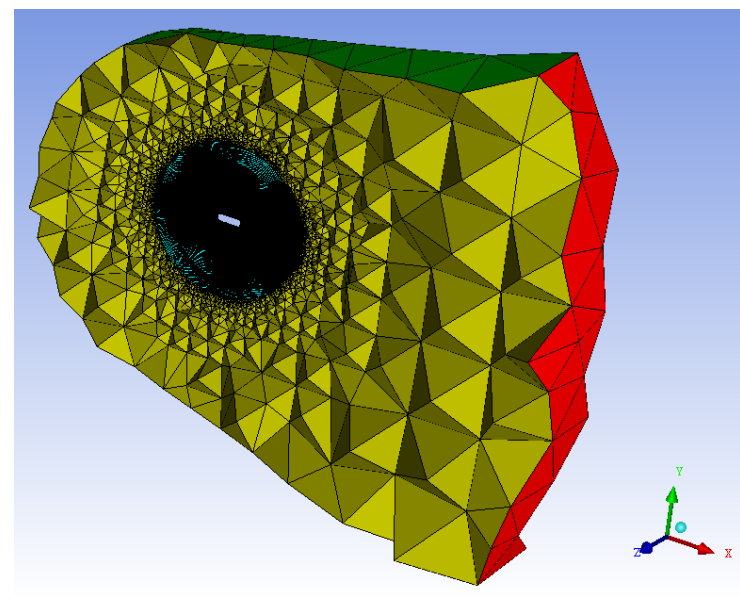

(a)

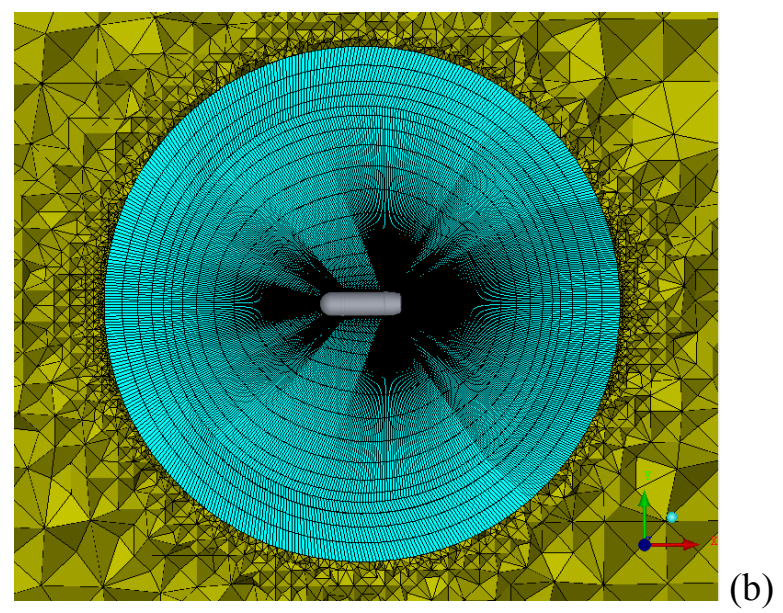

Figure 3-4: (a) Fluid Domains for $40 \mathrm{~mm}$ Baseline showing Velocity Inlet in Green and Pressure Outlet in Red; (b) Inner Fluid Domain Magnified to Show Unstructured Cells Growing Outward from Non-conformal Interface

\subsection{Tail-deployed Configuration}

The mesh for the tail-deployed configuration was similar to the baseline configuration in that the unstructured mesh was nearly identical. This, of course, was expected since the geometry of the inlet and outlet boundaries were the same, as provided in Figure 3-5. However, the structured mesh was different as a result of the tail fins, as shown in Figure 3-6. In order to properly create the structured mesh around the finned projectile, a C-grid was used to wrap the mesh around each fin. Therefore, this required more time and effort to obtain a mesh with acceptable quality. Figure 3-7 presents a front view of the finned projectile with the structured mesh wrapping around the body and tail fins. Three meshes of varying resolution were generated and the average number of nodes and cells were approximately 1,440,000 and $1,840,000$, respectively. 

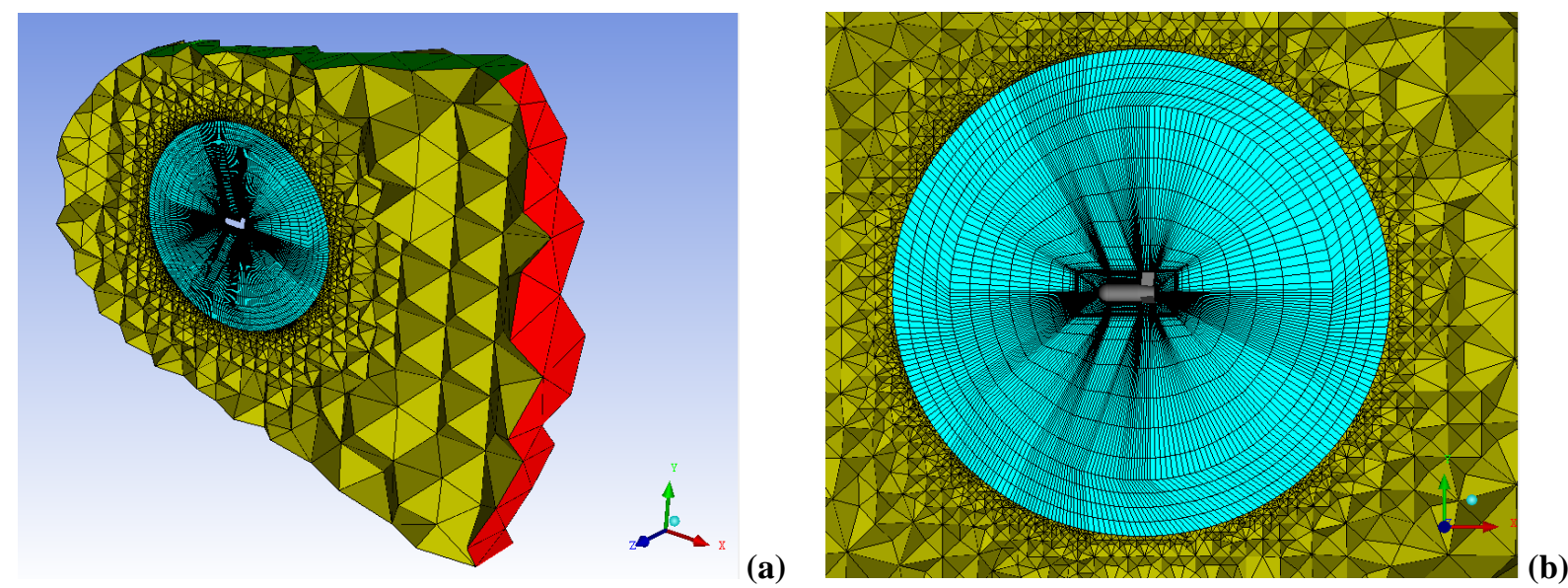

Figure 3-5: (a) Inner and Outer Fluid Domains for the Tail-deployed Configuration of the $\mathbf{4 0} \mathbf{~ m m}$ Projectile showing Velocity Inlet in Green and Pressure Outlet in Red; (b) Inner Fluid Domain Magnified to Show Unstructured Cells Growing Outward from Non-conformal Interface

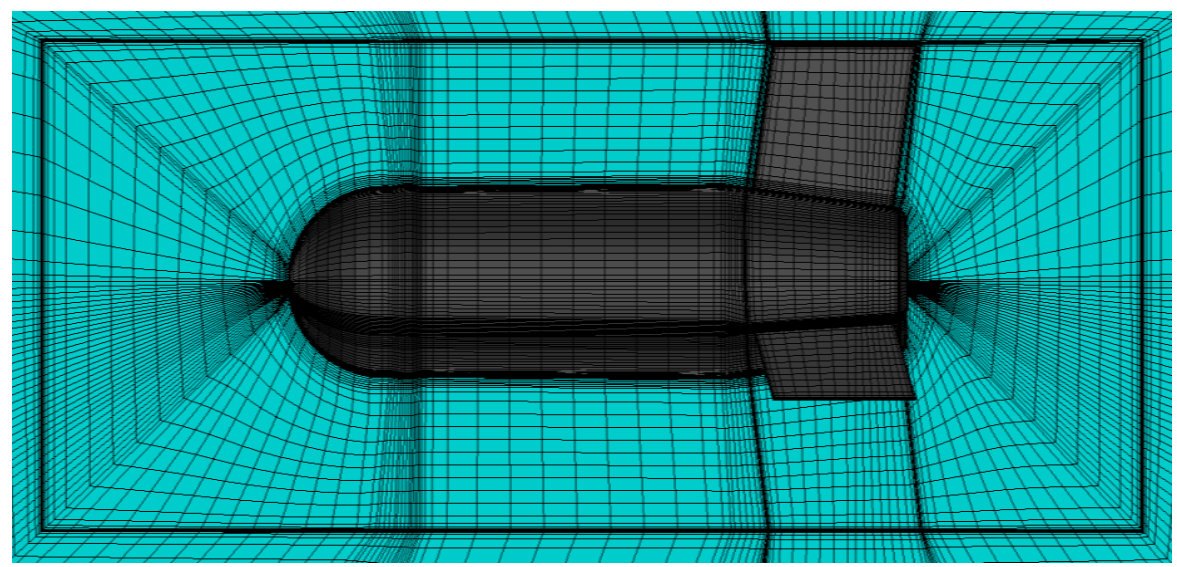

Figure 3-6: Medium Resolution Mesh of Fin-stabilized $40 \mathrm{~mm}$ Projectile.

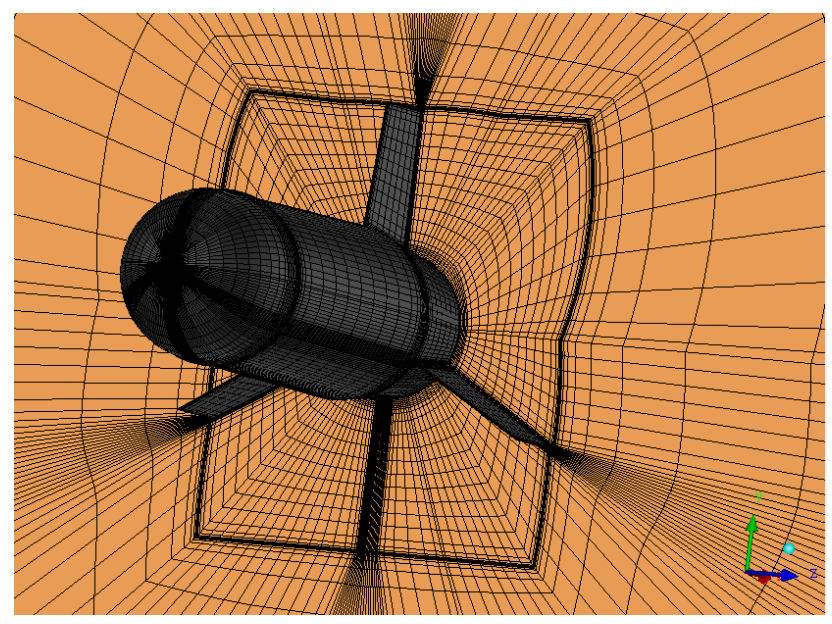

Figure 3-7: $40 \mathrm{~mm}$ Projectile Viewed from the Front Showing Mesh Wrapping around Body and Tail Fins. 


\subsection{Fully Deployed MAV Configuration}

Two designs of the fully deployed wing and tail configuration were considered. This included the Y-tail and inverted Y-tail configurations. As revealed in Figure 3-4 and Figure 3-5, the unstructured mesh design was nearly identical to the baseline and tail-deployed configurations. Beginning with the inverted Y-tail design, provided in Figure 3-8, the mesh required a considerable amount of time as compared to the mesh for the fin-stabilized projectile. The extra time and effort was attributed to creating C-grids around the wing in addition to creating O-grids and splits in the blocking structure. Just like the mesh for the finstabilized projectile, the idea behind generating C-grids around the wing surfaces was necessary to achieve acceptable mesh quality. Figure 3-9 and Figure 3-10 shows a view of the mesh for the fully deployed MAV configuration in which the mesh representing the inner fluid domain is wrapped around the body and wing. As provided in Figure 3-11, a side view of the MAV is shown with the mesh representing the inner fluid domain wrapping around the length of the body. Three meshes of varying resolution were generated and the average number of nodes and cells were approximately 1,763,000 and $2,150,000$, respectively.

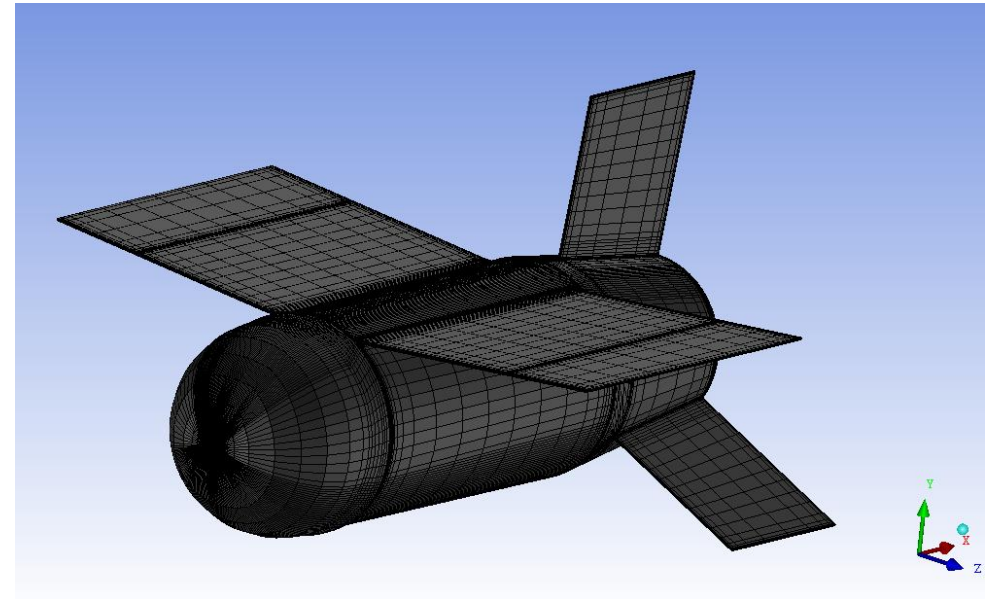

Figure 3-8: Medium Resolution Mesh of Wings-Deployed Configuration.

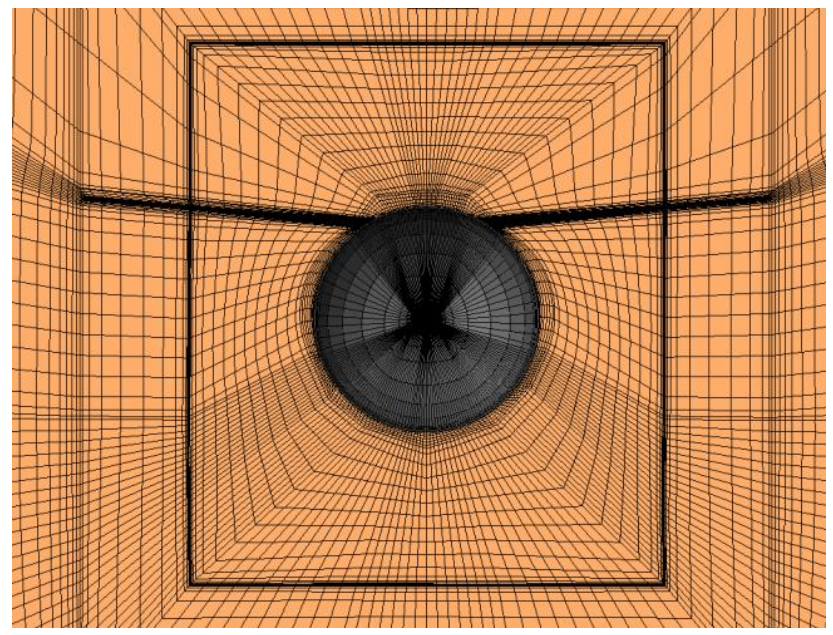

Figure 3-9: Wings-Deployed Configuration Showing Mesh Wrapped around Body and Wing. 


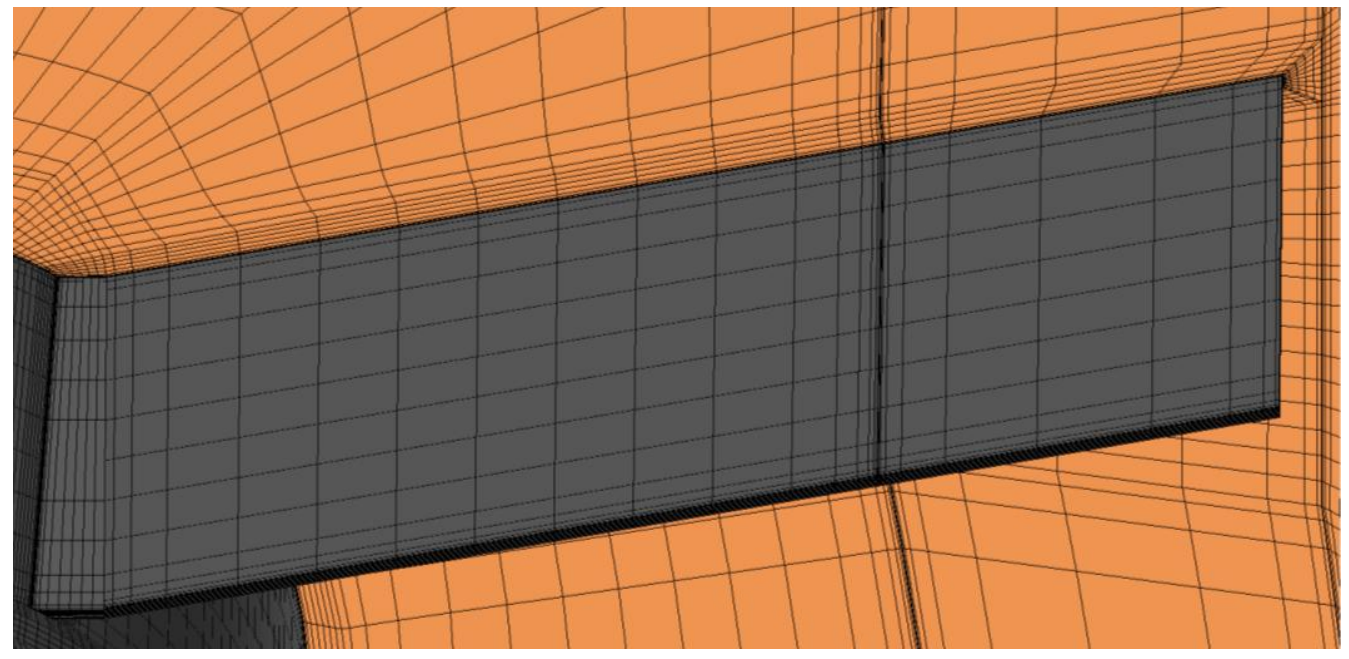

Figure 3-10: Fully Deployed MAV Magnified to Show C-grid Wrapped around Wing.

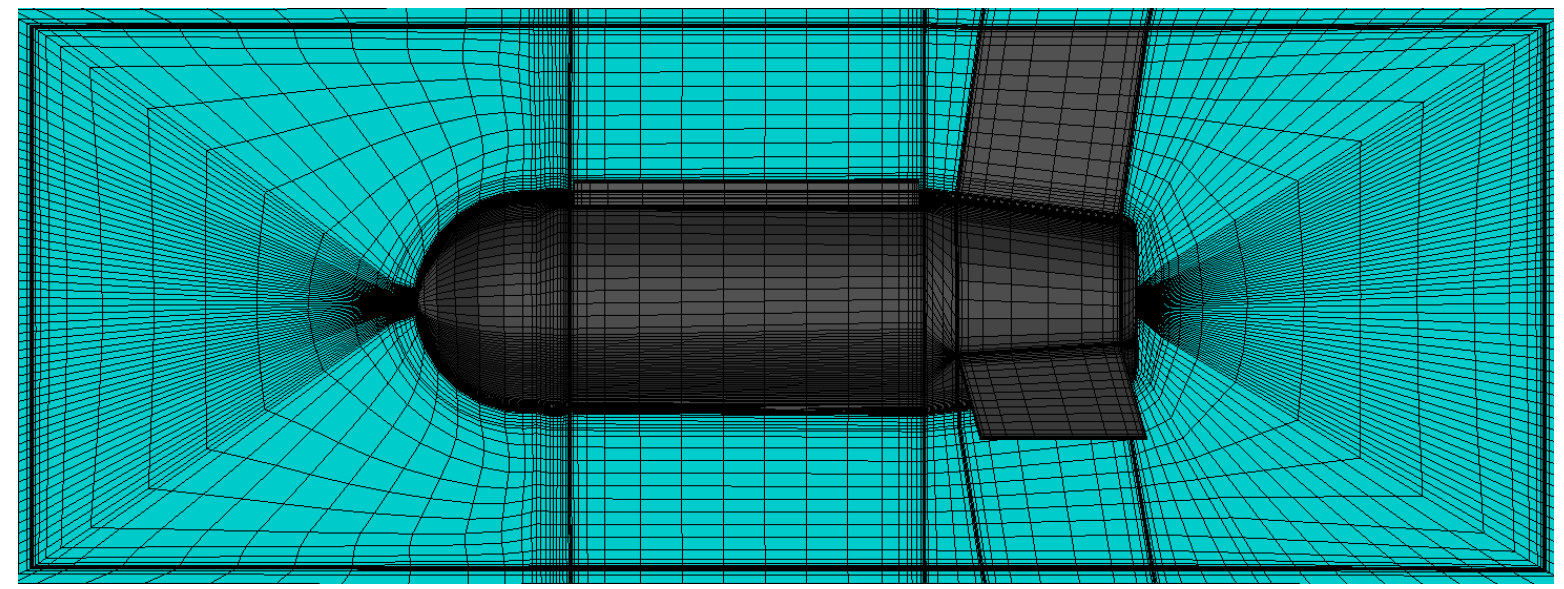

Figure 3-11: Inverted Y-tail Design of Wings-Deployed Configuration Showing Mesh Wrapped Lengthwise around Body.

For the alternative Y-tail configuration, a mesh independence study was not performed. Instead, a nearly identical node count to the medium resolution mesh of the inverted Y-tail configuration was generated. Since the geometry for the fully deployed MAV configurations only differed by a $180^{\circ}$ orientation of the tail, a significant change in the aerodynamic results would not be expected. As shown in Figure 3-12, a mesh was created to conduct a fixed body CFD analysis. In viewing the MAV from the front, it can be clearly shown that the tips of the two fins forming the $\mathrm{V}$-tail are directly in the path of the wake of the wing. As will be discussed later in this research, this resulted in instability issues in the longitudinal dynamics. Figure 3-13 provides a side view of the MAV in which the mesh representing the inner fluid domain is wrapped around the length of the body. 

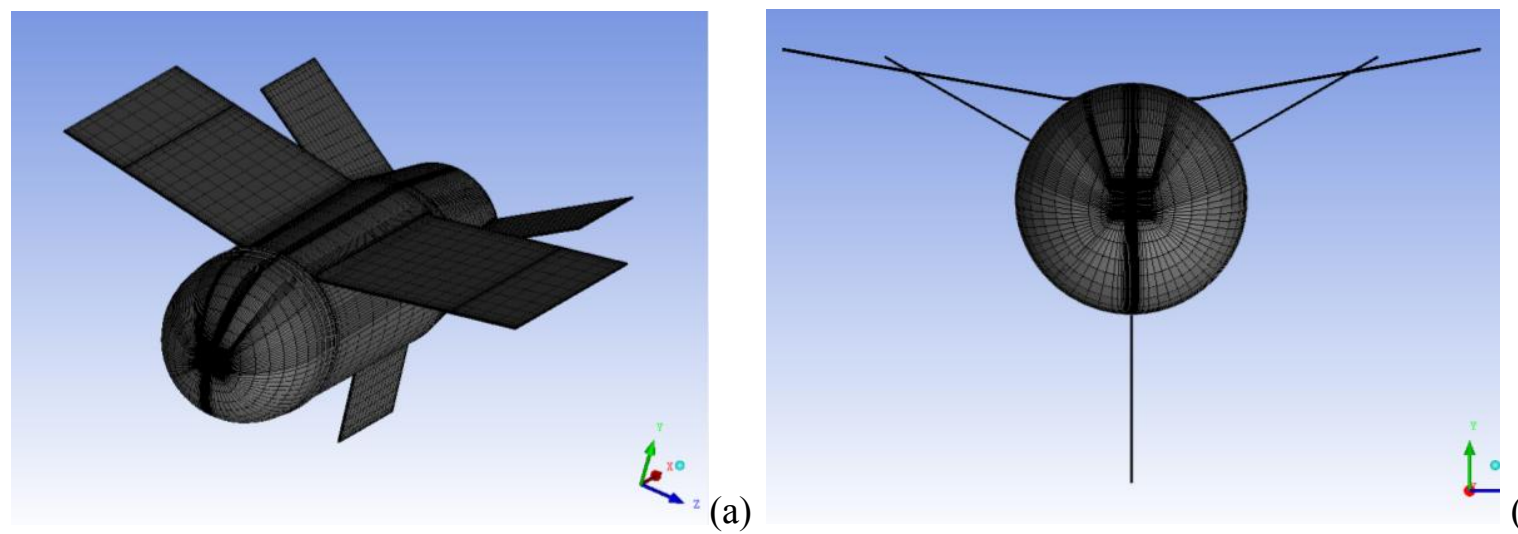

(a)

(b)

Figure 3-12: (a) Medium Resolution Mesh for Fully Deployed MAV; (b) MAV Viewed from the Front Showing V-tail in Wake of the Wing

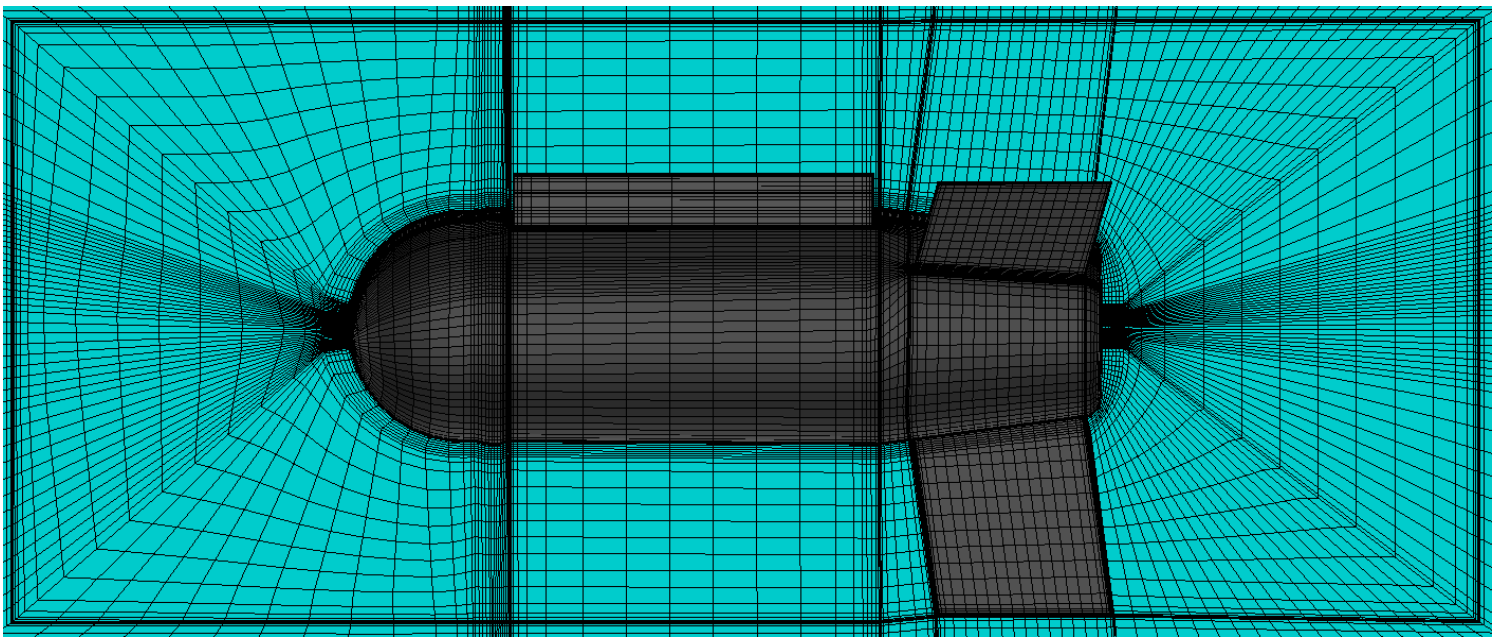

Figure 3-13: Y-tail Design of Fully Deployed MAV Configuration Showing Mesh Wrapped Lengthwise around Body. 


\section{Chapter 4 Fixed Body CFD Analysis}

The aerodynamic results for several traditional CFD simulations will be discussed for the baseline, tail-deployed, and wings-deployed configurations of the tube-launched transformable MAV. The motivation for conducting these simulations was to obtain a converged solution for the aerodynamic lift, drag, and pitching moment that was independent of mesh size. A converged solution was necessary before attempting to perform coupled CFD/6-DOF simulations since the computational time requirements for numerical flight testing were so great; these requirements will be discussed later. Before presenting the aerodynamic results for the three configurations, an overview of the boundary conditions and solver settings will be discussed in addition to the mesh independence studies.

\subsection{Boundary Conditions}

The boundary conditions were required for each of the three configurations of the tube-launched transformable MAV before executing the CFD simulations. These settings were configured such that the three geometries were fixed in space thereby constraining both translational and rotational motion. Beginning with the $40 \mathrm{~mm}$ baseline round, the fixed body CFD analysis was necessary in order to evaluate its static stability, otherwise known as gyroscopic stability. This was a necessary requirement in order for the projectile to have the possibility of being dynamically stable. Because the baseline round was launched at $70 \mathrm{~m} / \mathrm{s}$, the free stream velocity was set to the launch velocity. As explained previously in the hybrid mesh generation details, the outer surface of the unstructured mesh consisted of a velocity inlet for the defining the direction of the free stream velocity flow and a pressure outlet for allowing the airflow to exit the computational domain. Because the structured and unstructured cells were not conformal, a non-conformal interface boundary condition was required to compute the flux across the interface. At the surface of the baseline round, the fluid will have zero velocity relative to the surface and therefore the no-slip condition was applied.

The tail-deployed configuration imposed identical boundary conditions as described above for the baseline round. The free steam velocity was set to $70 \mathrm{~m} / \mathrm{s}$ since there would not be a considerable reduction in velocity upon deployment of the projectile's tail immediately after being launched from the tube. Nearly identical boundary conditions were applicable to the wings-deployed configuration. However, the free stream velocity was set to $45 \mathrm{~m} / \mathrm{s}$ instead. In the simple air trajectory analysis performed by Hamburg (2010), the calculations showed that for a $70 \mathrm{~m} / \mathrm{s}$ launch velocity and a $45^{\circ}$ launch angle, the apex velocity was approximately $45 \mathrm{~m} / \mathrm{s}$.

\subsection{Solver}

The solution methodology for the pressure-velocity solver was to use the Semi-Implicit Method for Pressure-Linked Equations Consistent (SIMPLEC) scheme. For complicated flows, such as the turbulence encountered in this research, the SIMPLEC algorithm can provide improved convergence over the standard SIMPLE scheme. The steady Reynolds Averaged Navier Stokes (RANS) solver was initially selected since it was less computationally expensive to run as compared to the unsteady RANS solver. For these traditional CFD simulations, the solution showed convergence using the steady solver and therefore, the unsteady solver was not considered since it would require considerably more time to obtain a solution. The method for calculating the gradients for the fixed body CFD simulations utilized the Green-Gauss cell-based discretization method. This was selected over the node-based discretization method because it was less computationally expensive. However, the cell-based method is known to be less accurate in computing the gradients for unstructured cells. Since this research was primarily concerned with the gradients in the inner fluid zone consisting of structured cells, the use of the cell-based discretization would not be an issue. For the first 100 iterations of the steady RANS solver, the spatial 
discretization for the pressure, momentum, turbulent kinetic energy, and specific dissipation rate were set to first order upwind. The justification for this approach was to obtain an approximate solution before switching the discretization to second order for a more accurate calculation. The solution did not require modifications to the under relaxation factors. For the initialization of the flow domain, the default settings were applied in which the velocity components were initialized to $0 \mathrm{~m} / \mathrm{s}$, turbulent kinetic energy to 1 $\mathrm{m}^{2} / \mathrm{s}^{2}$, and specific dissipation rate to $1 \mathrm{~s}^{-1}$.

\subsection{Mesh Independence Studies}

Mesh independence studies involved generating three different meshes having varying mesh resolution (coarse, medium, fine) and running simulations for each resolution at two different angles of attack (AOA). The idea behind performing these studies was to show that the computational results of aerodynamic lift, drag, and pitching moment converged as the mesh resolution increased. In other words, the results of the CFD simulations should become independent of mesh size.

For the baseline configuration, the mesh independence study showed that the aerodynamic lift, drag, and pitching moment converged for a medium resolution mesh consisting of approximately $1,192,000$ cells and 980,000 nodes. The overall cell quality of a mesh was essential to the accuracy and stability of the numerical computation. The magnitude in the overall cell quality ranged between zero and one in which these magnitudes represented the worst quality and the best quality, respectively. The calculated cell quality for the baseline mesh was 0.3, which was sufficient for ANSYS Fluent. As mentioned previously, the average $y^{+}$of less than 1 was necessary in order for the turbulence model to make accurate flow predictions. Figure 4-1 provides a plot of the wall $y^{+}$along the lengthwise distance of the $40 \mathrm{~mm}$ baseline round.

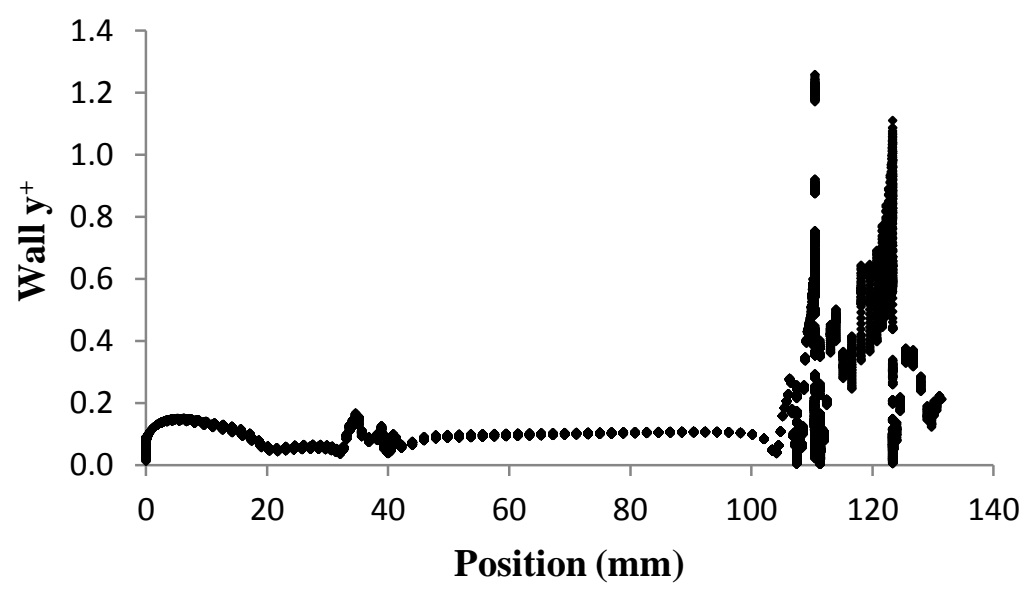

Figure 4-1: Wall $y^{+}$Plotted against the Lengthwise Distance of the Baseline Configuration

Prior to this research study, five experiments were performed using an induction wind tunnel at the Picatinny Arsenal facility to measure aerodynamic lift, drag, and pitching moment on the original baseline design having a total length of $80 \mathrm{~mm}$. Two tests were conducted at Mach 0.1 and Mach 0.2 and one test at Mach 0.3 for angles of attack ranging from $-4^{\circ}$ to $12^{\circ}$. The CFD analysis was conducted at Mach 0.2; however, the experimental drag showed a better comparison for Mach 0.3 at $0^{\circ}$ AOA with a measured drag coefficient of 0.158 . Therefore, results of the CFD analysis were compared to the data set for the Mach 0.3 case. The predicted drag compared quite well with the experimental drag, but as the angle of attack increased, the calculated drag was always being over-predicted in comparison to the measured drag. For $6^{\circ}$ AOA, the drag was over-predicted by approximately $11 \%$. In contrast to the drag predictions, the lift showed good comparisons at $0^{\circ}$ and $6^{\circ}$ AOA. The lift was considerably less over- 
predicted for $6^{\circ} \mathrm{AOA}$ with an error of approximately 3\%. Table 4-1 provides the lift and drag for the coarse, medium, and fine meshes at $0^{\circ}$ and $6^{\circ}$ AOA. Figure 4-2 and Figure 4-3 illustrates the convergence of the lift and drag, respectively.

Table 4-1: Lift and Drag Data for Grid Independence Study of Baseline Configuration

\begin{tabular}{|c|c|c|c|c|c|c|}
\hline Mesh Resolution & \multicolumn{2}{|c|}{ Coarse } & \multicolumn{2}{c|}{ Medium } & \multicolumn{2}{c|}{ Fine } \\
\hline Nodes & \multicolumn{2}{|c|}{684,000} & \multicolumn{2}{c|}{984,000} & \multicolumn{2}{c|}{$1,556,000$} \\
\hline Cells & \multicolumn{2}{|c|}{874,000} & \multicolumn{2}{c|}{$1,192,000$} & \multicolumn{2}{|c|}{$1,882,000$} \\
\hline AOA (deg) & $\mathrm{C}_{\mathrm{D}}$ & $\mathrm{C}_{\mathrm{L}}$ & $\mathrm{C}_{\mathrm{D}}$ & $\mathrm{C}_{\mathrm{L}}$ & $\mathrm{C}_{\mathrm{D}}$ & $\mathrm{C}_{\mathrm{L}}$ \\
\hline 0 & 0.1644 & -0.0038 & 0.159 & 0.0017 & 0.155 & 0.0005 \\
\hline 6 & 0.2181 & 0.1658 & 0.207 & 0.1611 & 0.202 & 0.1609 \\
\hline
\end{tabular}

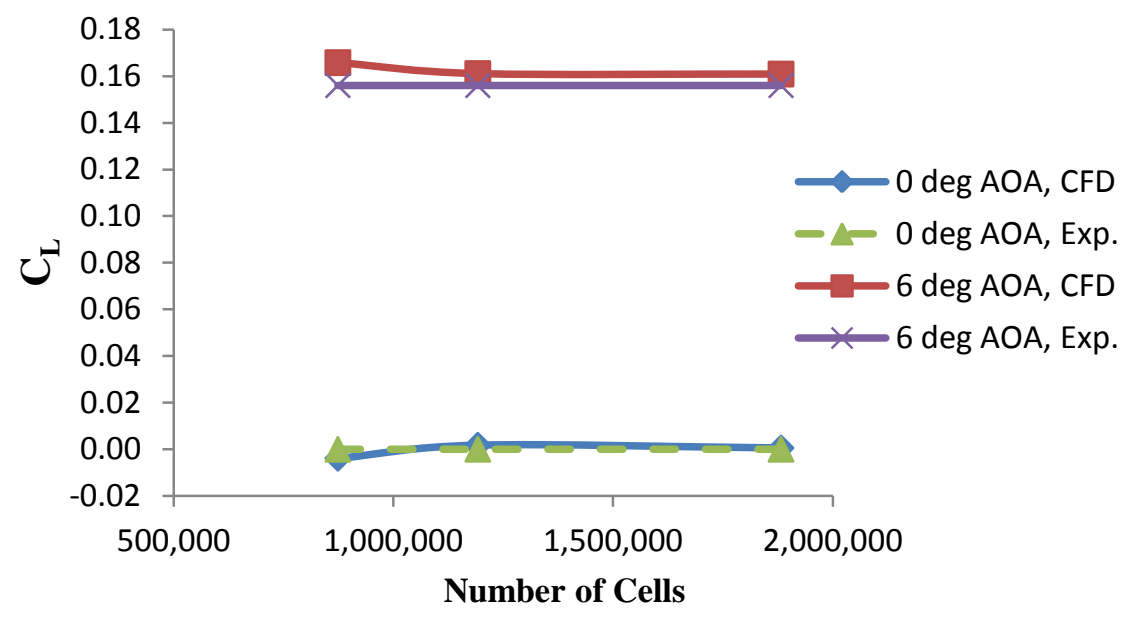

Figure 4-2: Grid Independence for Lift Data on Baseline Configuration

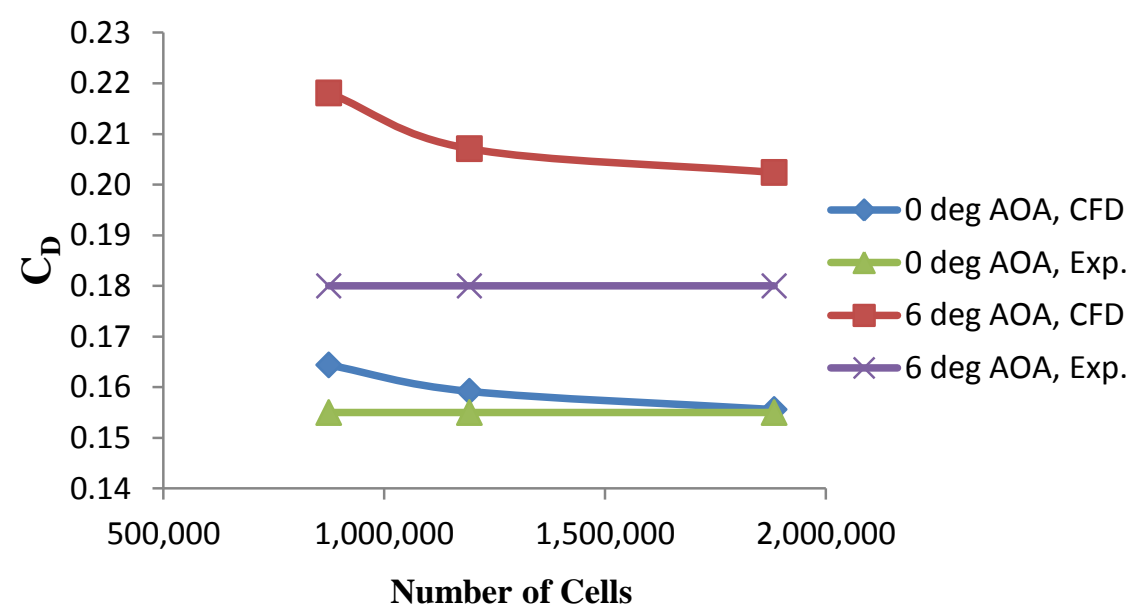

Figure 4-3: Grid Independence for Drag Data on Baseline Configuration 
Because the numerical drag on the baseline round was over-predicted for increasing angles of attack, the drag on the tail-deployed configuration was expected to be over-predicted as well. This statement can be justified by that fact that the main projectile body is geometrically similar to the baseline round and that the k- $\omega$ SST turbulence model remains unchanged. However, no experimental data was available for comparison. After performing a mesh independence study on the tail-deployed configuration, the predicted drag for $0^{\circ}$ and $6^{\circ}$ AOA was 0.179 and 0.268 , respectively. The increase in drag can be attributed to the additional skin-friction drag on the surface of the three tail fins. The results became independent of mesh size for a medium resolution mesh consisting of 1,662,000 cells and $1,269,000$ nodes. The cell quality for the tail-deployed configuration was 0.2 , which was less than the cell quality of the baseline round as a result of the more complex structured mesh wrapped around the tail fins. Nonetheless, the mesh quality was sufficient for ANSYS Fluent. Table 4-2 provides the lift and drag for the three varying mesh sizes at $0^{\circ}$ and $6^{\circ}$ AOA. Figure 4-4 and Figure 4-5 show the convergence of the lift and drag, respectively. In comparison to the fine resolution mesh, the numerical drag was overpredicted by $9 \%$ when running the solution using the coarse mesh at $0^{\circ} \mathrm{AOA}$ and under-predicted by $0.5 \%$ using the medium mesh. For $6^{\circ} \mathrm{AOA}$, the drag was over-predicted by $6 \%$ using the coarse mesh and dropped to $1.5 \%$ over-prediction using the medium mesh. There was not considerable change in the lift between the various mesh sizes for both $0^{\circ}$ and $6^{\circ}$ AOA. Figure 4-6 shows the wall $y^{+}$along the lengthwise distance of the tail-deployed configuration in which the average $y^{+}$was less than 1 .

Table 4-2: Lift and Drag Data for Grid Independence Study of Tail-deployed Configuration

\begin{tabular}{|c|c|c|c|c|c|c|}
\hline Mesh Resolution & \multicolumn{2}{|c|}{ Coarse } & \multicolumn{2}{c|}{ Medium } & \multicolumn{2}{c|}{ Fine } \\
\hline Nodes & \multicolumn{2}{|c|}{874,000} & \multicolumn{2}{c|}{$1,269,000$} & \multicolumn{2}{c|}{$2,184,000$} \\
\hline Cells & \multicolumn{2}{|c|}{$1,154,000$} & \multicolumn{2}{c|}{$1,662,000$} & \multicolumn{2}{|c|}{$2,705,000$} \\
\hline AOA (deg) & $C_{D}$ & $C_{L}$ & $C_{D}$ & $C_{L}$ & $C_{D}$ & $C_{L}$ \\
\hline 0 & 0.196 & -0.0041 & 0.179 & -0.0122 & 0.180 & 0.0079 \\
\hline 6 & 0.279 & 0.7620 & 0.268 & 0.7724 & 0.264 & 0.7713 \\
\hline
\end{tabular}

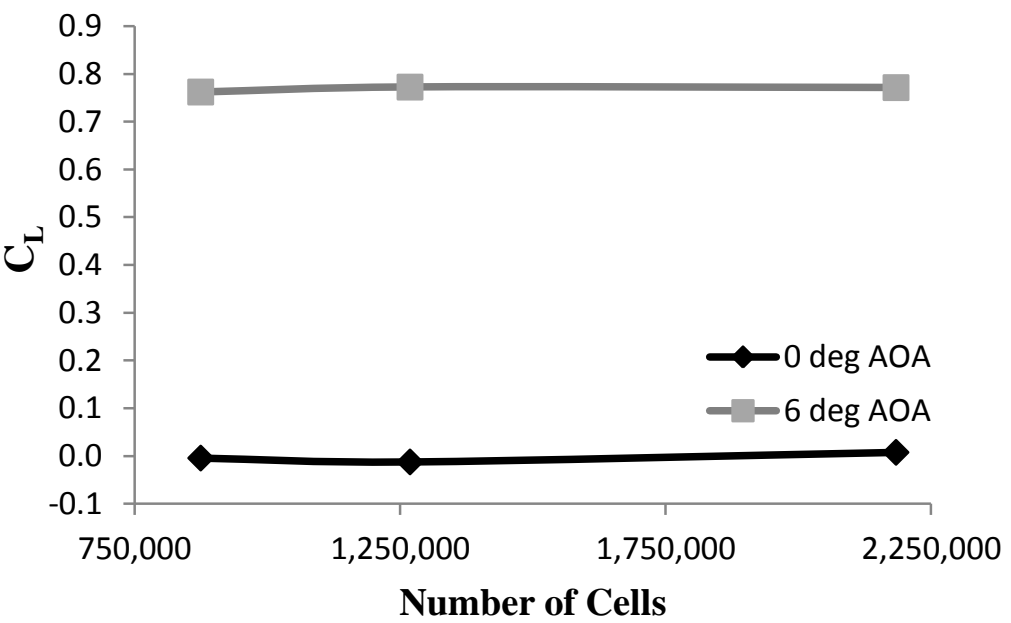

Figure 4-4: Grid Independence for Lift Data on Tail-deployed Configuration 


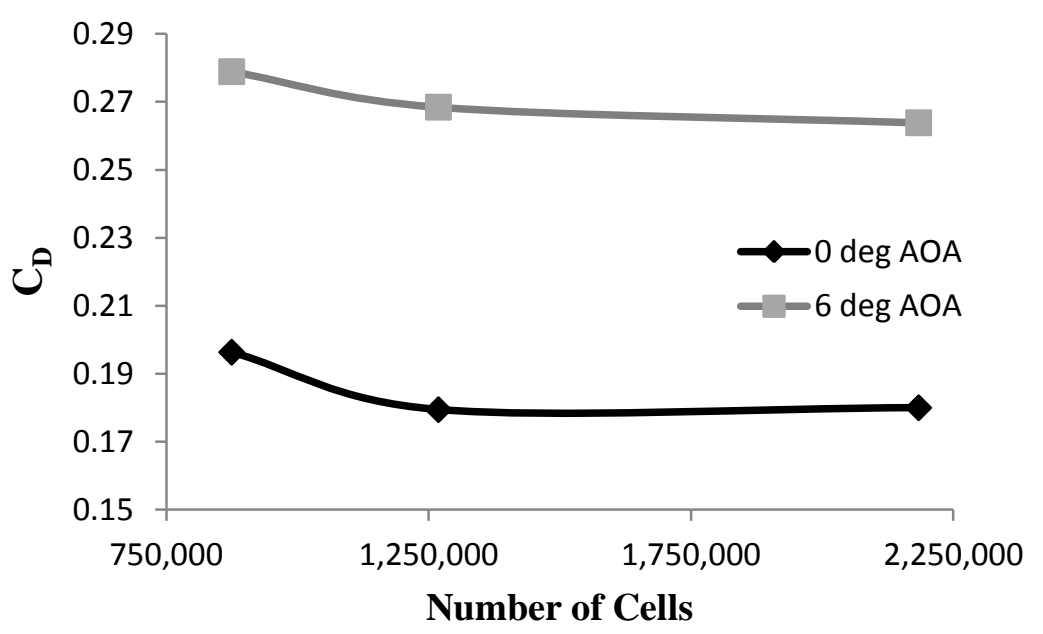

Figure 4-5: Grid Independence for Drag Data on Tail-deployed Configuration

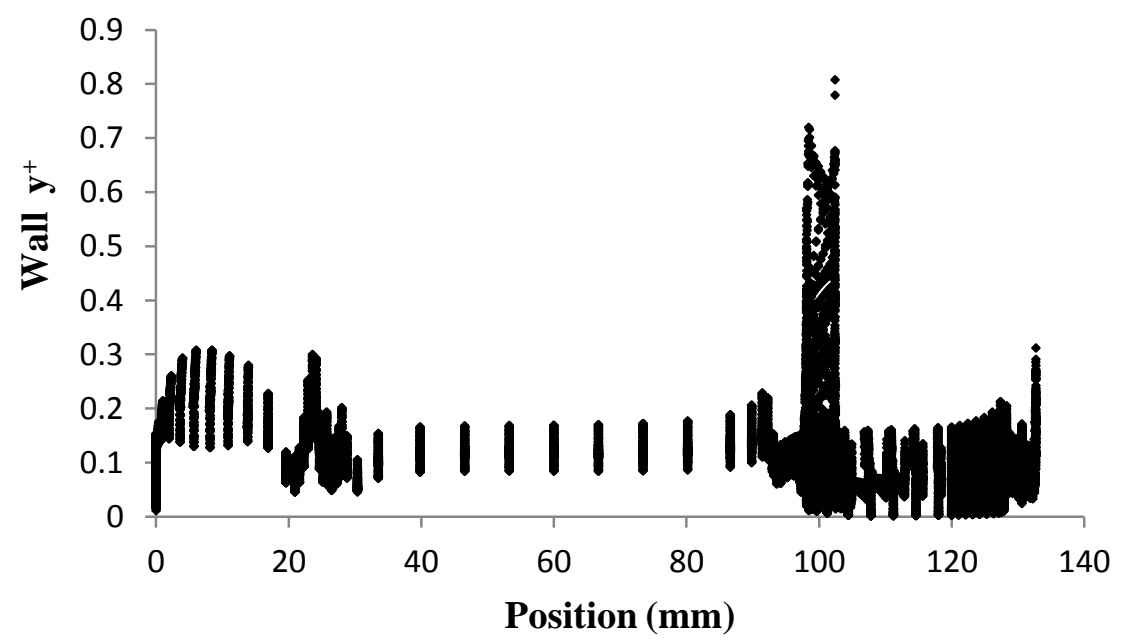

\section{Figure 4-6: Wall $y^{+}$Plotted against the Lengthwise Distance of the Tail-deployed Configuration}

Similar to the discussion with the fin-stabilized projectile, the numerical drag on the wingsdeployed configuration was expected to be over-predicted as well. The drag converged to a value of 0.245 and 0.490 for $0^{\circ}$ and $6^{\circ} \mathrm{AOA}$, respectively. In comparison to the fin-stabilized projectile, the drag on the wings-deployed configuration was considerably greater, which can be attributed to the wings generating additional lift induced drag as well as additional surface area for skin-friction drag. A mesh independence study was performed on the inverted Y-tail design of the wings-deployed configuration. Mesh independence was achieved for a medium resolution mesh consisting of 1,954,000 cells and 1,579,000 nodes. Table 4-3 provides the numerical results for each of the three different mesh sizes. The overall cell quality for the medium resolution mesh was 0.15 , which can be attributed to the region of cells near the root of the wings. In comparing the numerical results of the fine resolution mesh, the calculated drag at $0^{\circ}$ AOA was over-predicted by $18 \%$ using the coarse mesh and then dropped significantly to $1.6 \%$ using the medium mesh. For $6^{\circ} \mathrm{AOA}$, the drag was over-predicted by $8.2 \%$ using the coarse mesh and dropped considerably to $2 \%$ using the medium mesh. There was no considerable change in lift for $0^{\circ}$ or $6^{\circ} \mathrm{AOA}$. Figure 4-7 and Figure 4-8 shows the convergence of lift and drag, respectively. As stated previously, a mesh independence study was not performed on the Y-tail configuration in order to conserve time. Instead, a mesh was generated with nearly identical cell count to match the medium resolution mesh of 
the inverted Y-tail design. Figure 4-9 shows the wall $y^{+}$along the lengthwise distance of the wingsdeployed configuration. The average $y^{+}$was less than 1 with the largest values located at the root of the wing's leading edge.

Table 4-3: Lift and Drag Data for Grid Independence Study of Fully Deployed MAV Configuration

\begin{tabular}{|c|c|c|c|c|c|c|}
\hline Mesh Resolution & \multicolumn{2}{|c|}{ Coarse } & \multicolumn{2}{c|}{ Medium } & \multicolumn{2}{c|}{ Fine } \\
\hline Nodes & \multicolumn{2}{|c|}{974,000} & \multicolumn{2}{c|}{$1,579,000$} & \multicolumn{2}{c|}{$2,738,000$} \\
\hline Cells & \multicolumn{2}{|c|}{$1,231,000$} & \multicolumn{2}{|c|}{$1,954,000$} & \multicolumn{2}{|c|}{$3,276,000$} \\
\hline AOA (deg) & $\mathrm{C}_{\mathrm{D}}$ & $\mathrm{C}_{\mathrm{L}}$ & $\mathrm{C}_{\mathrm{D}}$ & $\mathrm{C}_{\mathrm{L}}$ & $\mathrm{C}_{\mathrm{D}}$ & $\mathrm{C}_{\mathrm{L}}$ \\
\hline 0 & 0.293 & -0.064 & 0.245 & -0.045 & 0.249 & -0.055 \\
\hline 6 & 0.529 & 2.209 & 0.498 & 2.280 & 0.489 & 2.260 \\
\hline
\end{tabular}

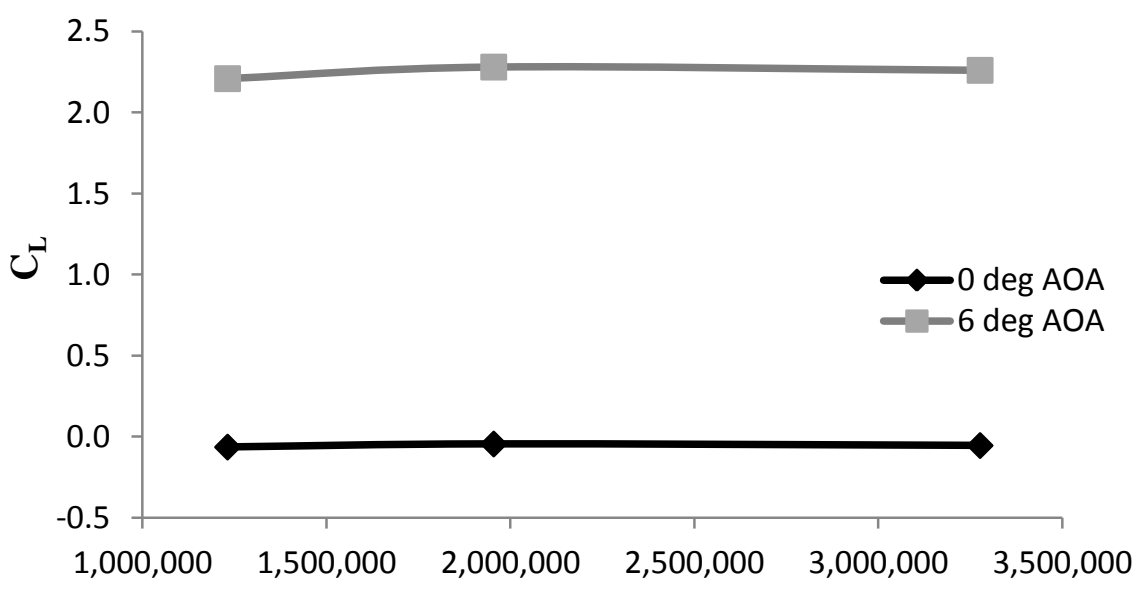

Number of Cells

Figure 4-7: Grid Independence for Lift Data on Fully Deployed MAV Configuration

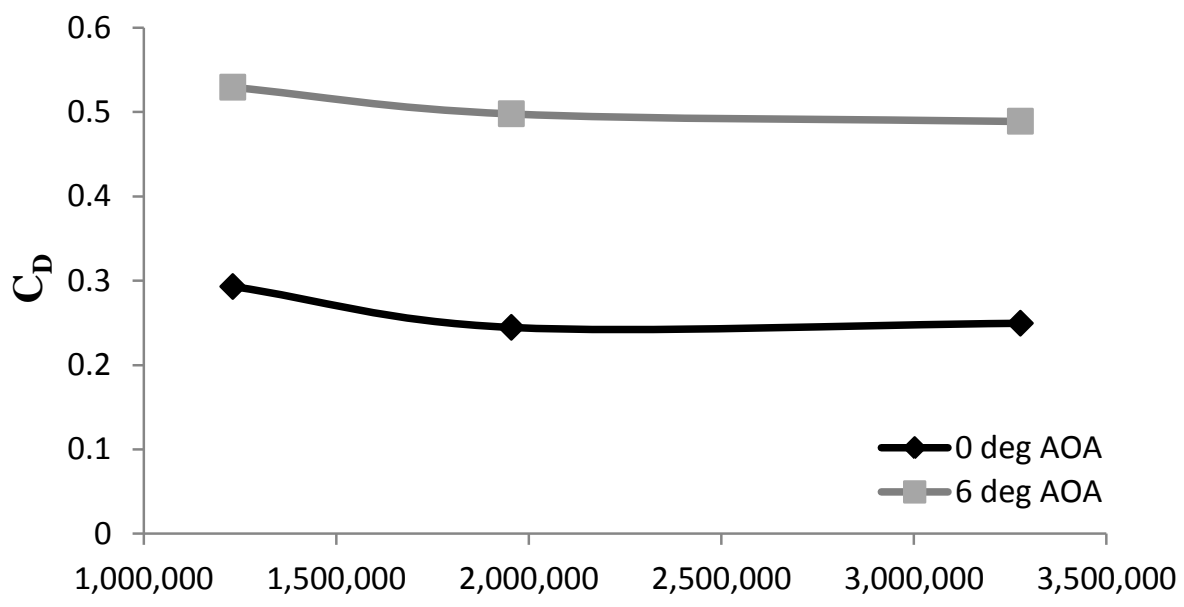

Number of Cells

Figure 4-8: Grid Independence for Drag Data on Fully Deployed MAV Configuration 


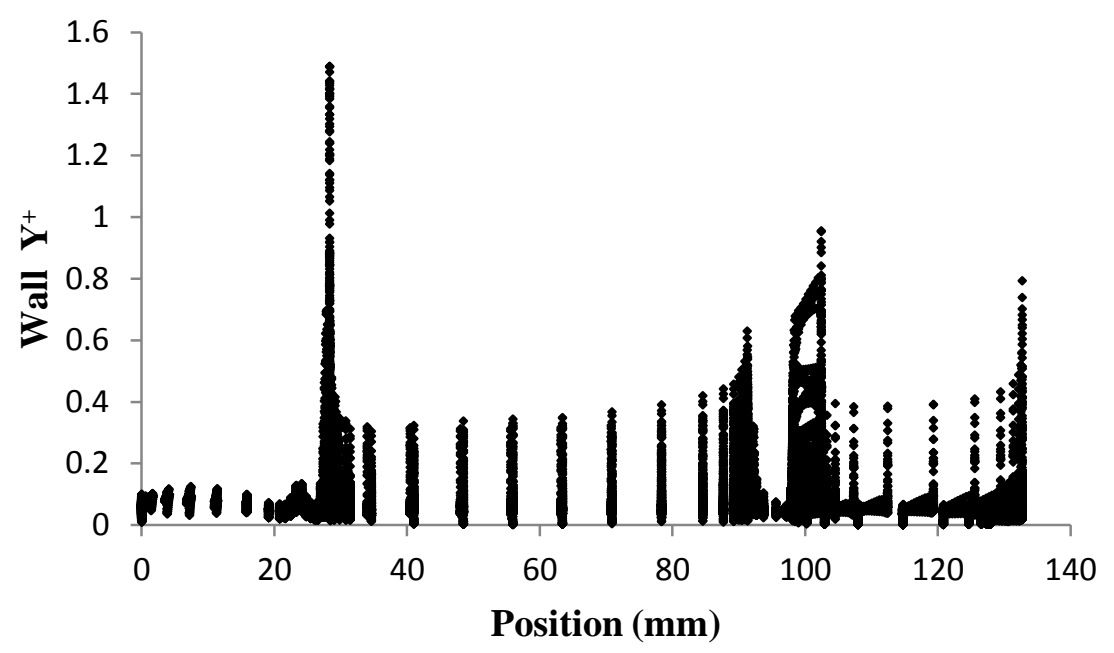

Figure 4-9: Wall $y^{+}$Plotted against the Lengthwise Distance of the Fully Deployed MAV Configuration

\subsection{Simulation Results}

\subsubsection{Baseline Configuration}

Before performing an analysis of the $40 \mathrm{~mm}$ round undergoing free body motion, a study of the flow physics around the projectile body while fixed in space was necessary for evaluating its gyroscopic stability. Static stability was essential for the projectile to be dynamically stable. This was noted in Figure 2-23, which illustrated the regions of dynamic stability and dynamic instability for a spinning projectile.

Using the medium resolution mesh, the CFD analysis was performed for angles of attack ranging from $-10^{\circ}$ to $10^{\circ}$. Figure 4-10 provides the CFD results of the lift curve slope for a free stream velocity of $70 \mathrm{~m} / \mathrm{s}$ or Mach 0.2 and compares it with the measured data obtained from the wind tunnel tests introduced in the previous section. As explained in the mesh independence study for the baseline round, the lift computed from CFD showed good comparison with the lift obtained from the wind tunnel tests. Similarly, Figure 4-11 provides a comparison of predicted and experimentally measured drag. In contrast to the lift, the drag was over-predicted for increasing angles of attack. The experimental data showed that the k- $\omega$ SST turbulence model over-predicted the drag coefficient by as much as $20 \%$. However, in an engineering point of view, the use of this turbulence model was a more conservative approach. In addition to the lift and drag, the pitching moment was also calculated from the CFD analysis. In the simulation, the pitching moment was computed at the hemispherical nose of the projectile and was then recalculated at the CG location. Figure 4-12 illustrates the location of the pitching moment at the nose and CG of the 40 $\mathrm{mm}$ baseline round as well as illustrations of the lift and drag forces. This figure was used to help visualize how to solve for the moment at the $\mathrm{CG}$ of the baseline round using Equation 4-1. 


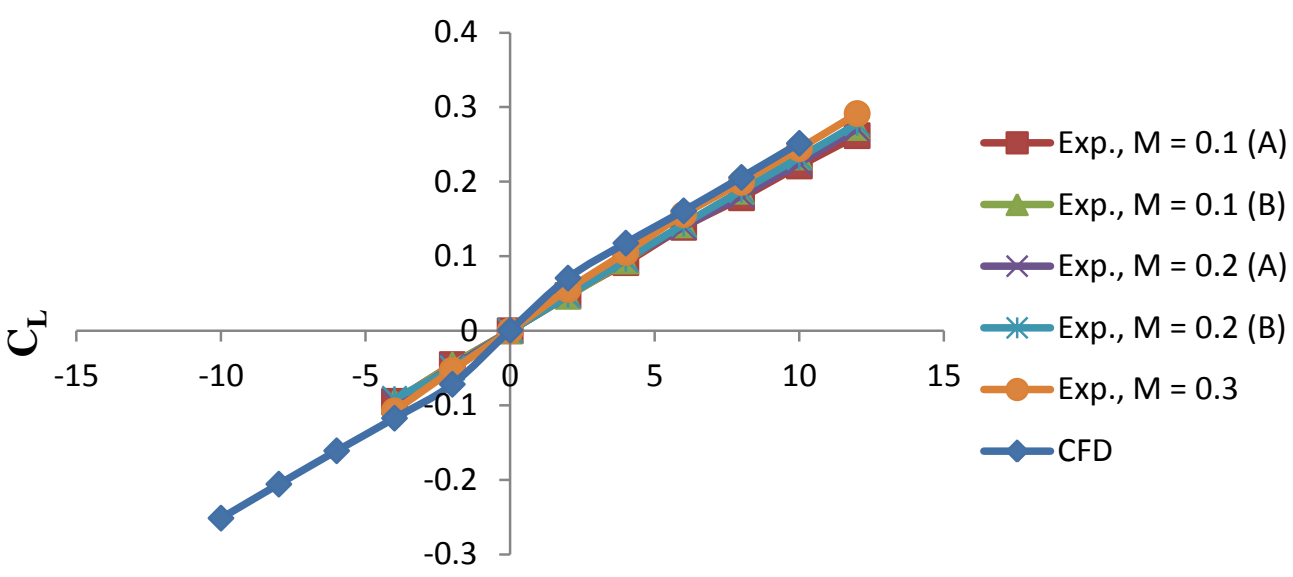

Angle of Attack (deg)

Figure 4-10: Comparison of Aerodynamic Lift for $40 \mathrm{~mm}$ Baseline Configuration

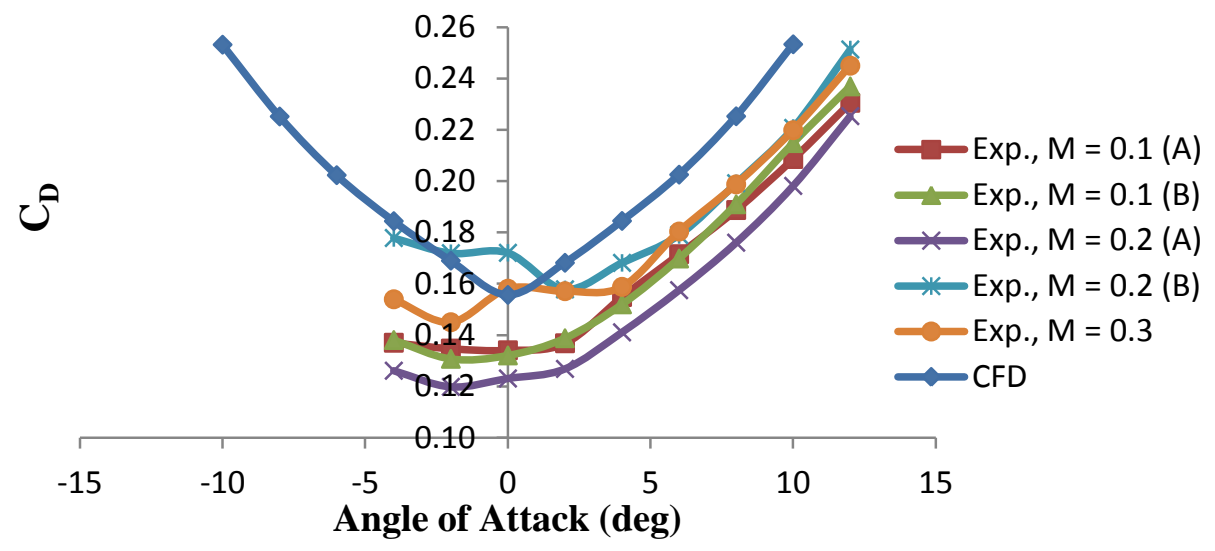

Figure 4-11: Comparison of Aerodynamic Drag for $40 \mathrm{~mm}$ Baseline Configuration

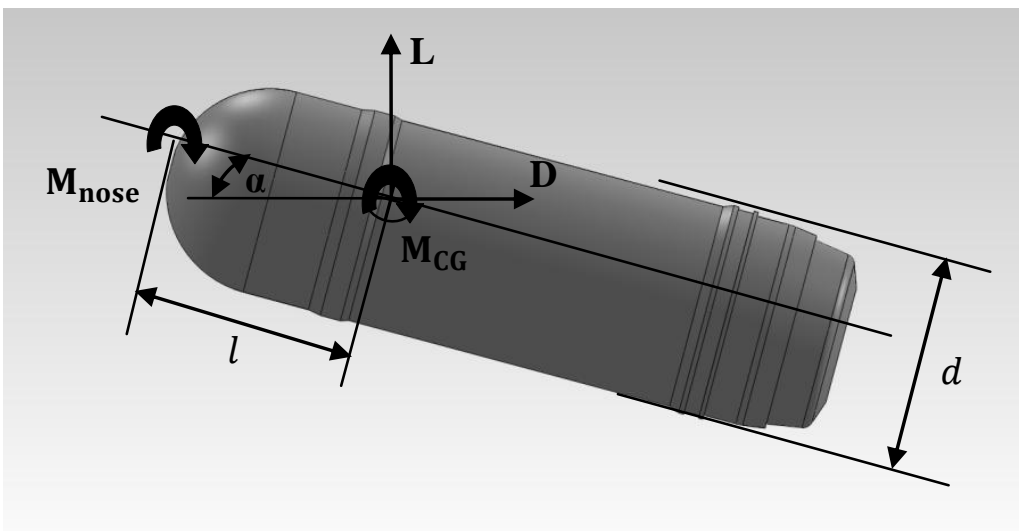

Figure 4-12: Illustration of Aerodynamic Forces and Moments Acting on $40 \mathrm{~mm}$ Round

$$
C_{m_{C G}}=C_{m_{\text {nose }}}+\left(C_{L} \cos \alpha+C_{D} \sin \alpha\right) \frac{l}{d}
$$

Figure 4-13 provides a comparison of the experimental and numerical data for the pitching moment about the CG location. By fitting the data points linearly, the slope of the curve or pitching moment coefficient derivative, $C_{m_{\alpha}}$, was approximately 0.0197 per degree from the CFD analysis and 
0.0193 per degree from wind tunnel testing. The numerical $C_{m_{\alpha}}$ derivative was determined within 2 percent of the experimental $C_{m_{\alpha}}$. Using this derivative, the gyroscopic stability factor, $S_{g}$, could be evaluated using Equation 2-28. According to the CFD simulations and experimental testing, the magnitude of $S_{g}$ was approximately equal to 35 . Because this factor was considerably greater than 1 , it indicated that the $40 \mathrm{~mm}$ round was gyroscopically stable; however, this would not guarantee dynamic stability.

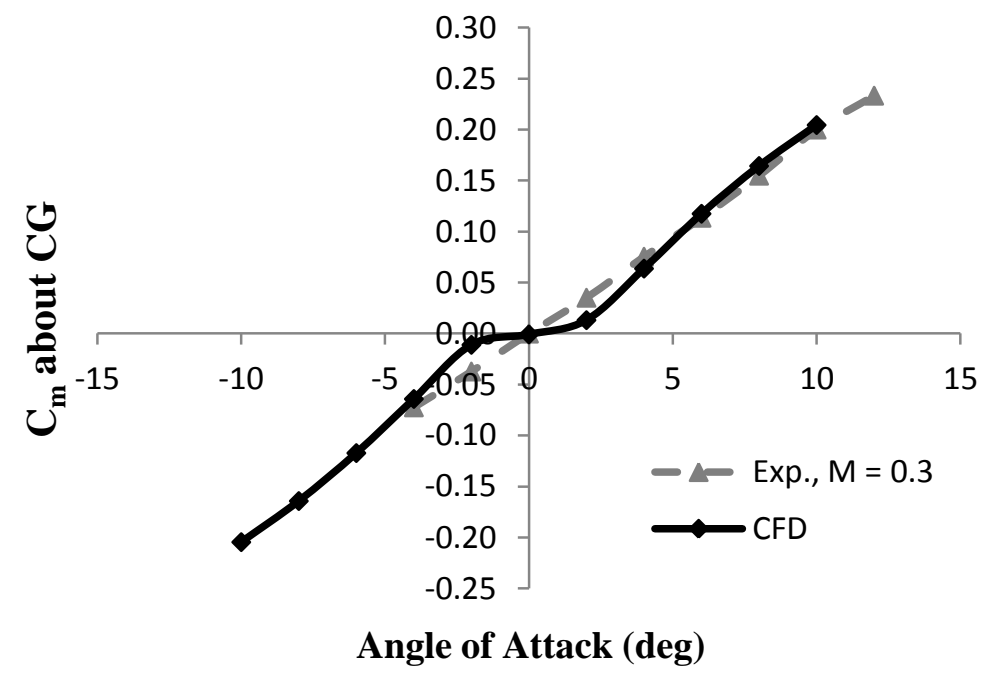

Figure 4-13: Experimental and Computational Comparison of Pitching Moment about CG of $40 \mathrm{~mm}$ Baseline

The results of the simulations for the baseline were examined. As shown in Figure 4-14, the contours of velocity magnitude were plotted for the $40 \mathrm{~mm}$ round at $0^{\circ} \mathrm{AOA}$, in which the flow was axisymmetric. As expected, a stagnation region was present at the hemispherical nose tip. This was indicated by the maximum pressure coefficient having a magnitude of 1 as shown in the contours of Figure 4-15. Also, these contours confirmed that the flow was axisymmetric and consequently no lift was generated on the body. Since the free stream flow was subsonic, the airspeed was reduced upstream from the hemispherical because the flow was anticipating the projectile body, as shown in the contours of velocity magnitude. As the airflow turned around the hemispherical nose, it reached a maximum velocity of $90.5 \mathrm{~m} / \mathrm{s}$ and thus, the entire flow domain remained within the acceptable incompressible assumption limit. Figure 4-16 provides the pathlines of velocity magnitude that clearly shows a region of recirculating flow within the obturator groove as well as the recirculation aft of the backward facing steps. As the flow passed the rear casing, the flow turned and separated. This created an area of low pressure aft of the body in which two regions of recirculating flow were generated, as shown in Figure 4-17. However, the flow characteristics became asymmetrical at other angles of attack. At $6^{\circ}$ AOA, the flow asymmetry can be clearly shown by displaying the contours of pressure coefficient provided in Figure 4-18. A smaller adverse pressure gradient on the leeward side allowed the air to flow faster than on the windward side. As the air reached the slanted rear casing, the turning angle was greater on the leeward side and the airflow showed separation, whereas the flow on the windward side had a smaller turning angle and thus allowed the flow to expand. The velocity pathlines are displayed in Figure 4-19 to show the faster flow speed passing over the hemispherical nose on the leeward side as well as the flow separation aft of the body. The higher pressure on the windward side and the lower pressure on the leeward side accounted for the lift that was generated on the body. 


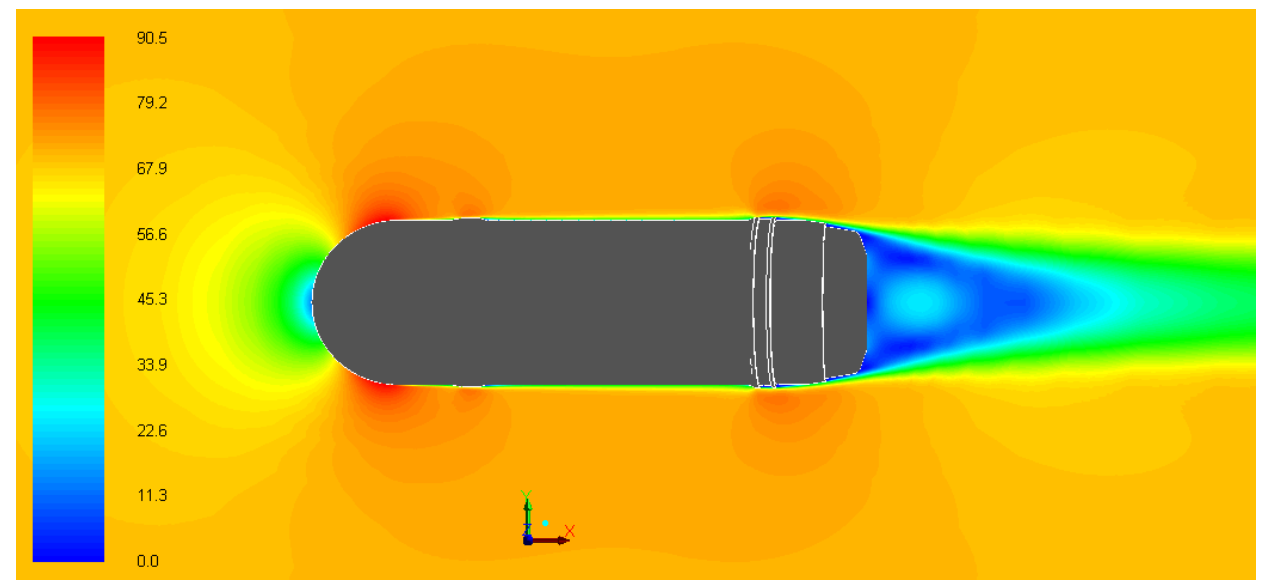

Figure 4-14: Contours of Velocity Magnitude for $40 \mathrm{~mm}$ Round at $0^{\circ} \mathrm{AOA}$

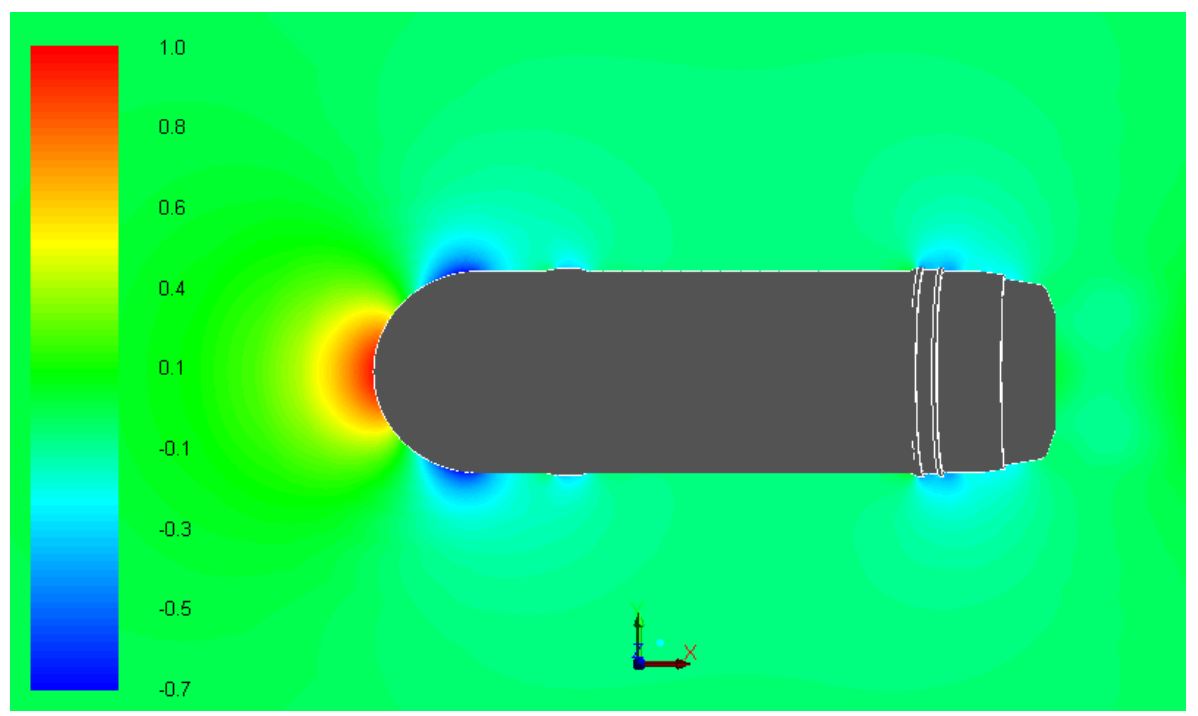

Figure 4-15: Contours of Pressure Coefficient for $40 \mathrm{~mm}$ Round at $0^{\circ} \mathrm{AOA}$

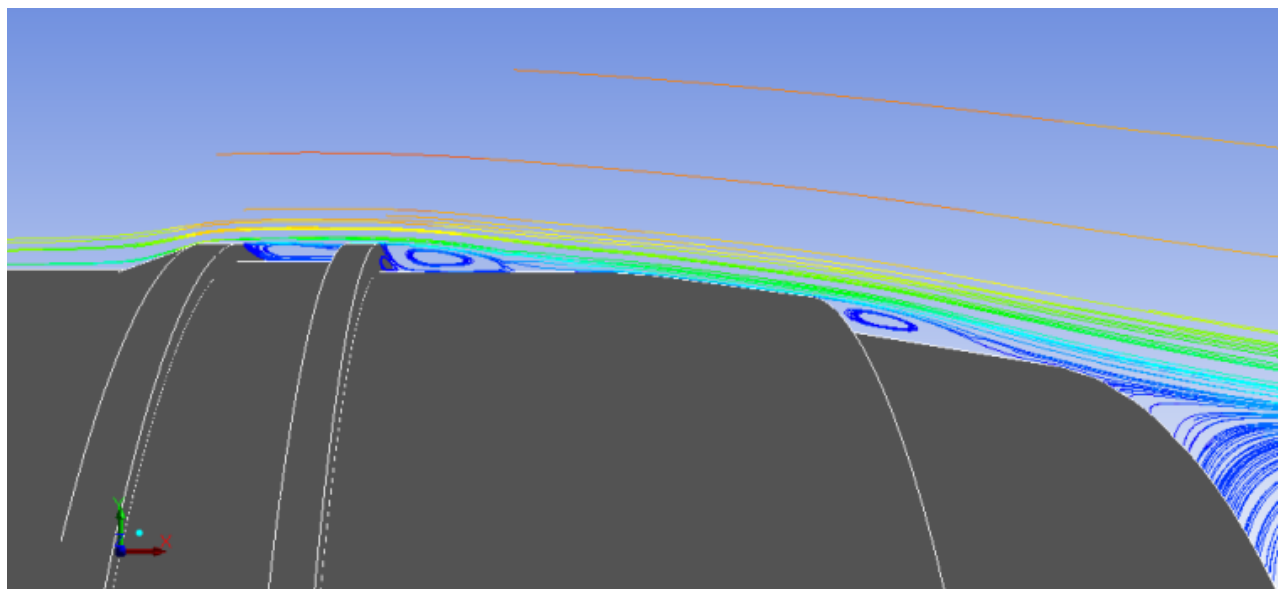

Figure 4-16: Velocity Pathlines Showing Recirculation in Obturator Groove and Rear Casing 


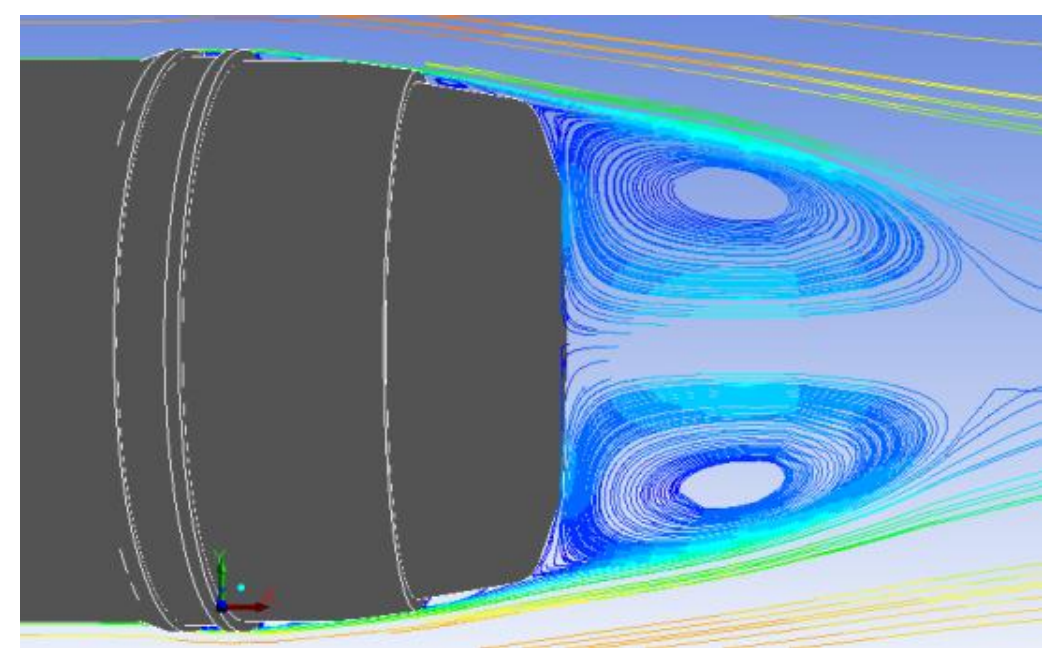

Figure 4-17: Velocity Pathlines Showing Recirculation Regions Aft of the $40 \mathrm{~mm}$ Round

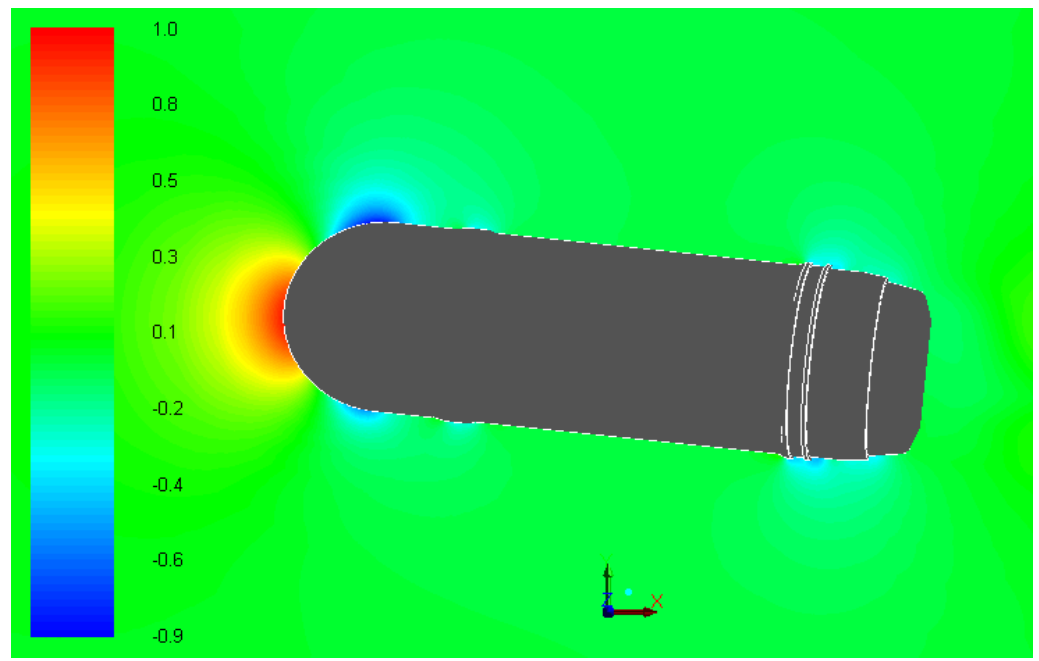

Figure 4-18: Contours of Pressure Coefficient for $40 \mathrm{~mm}$ Round at $6^{\circ} \mathrm{AOA}$

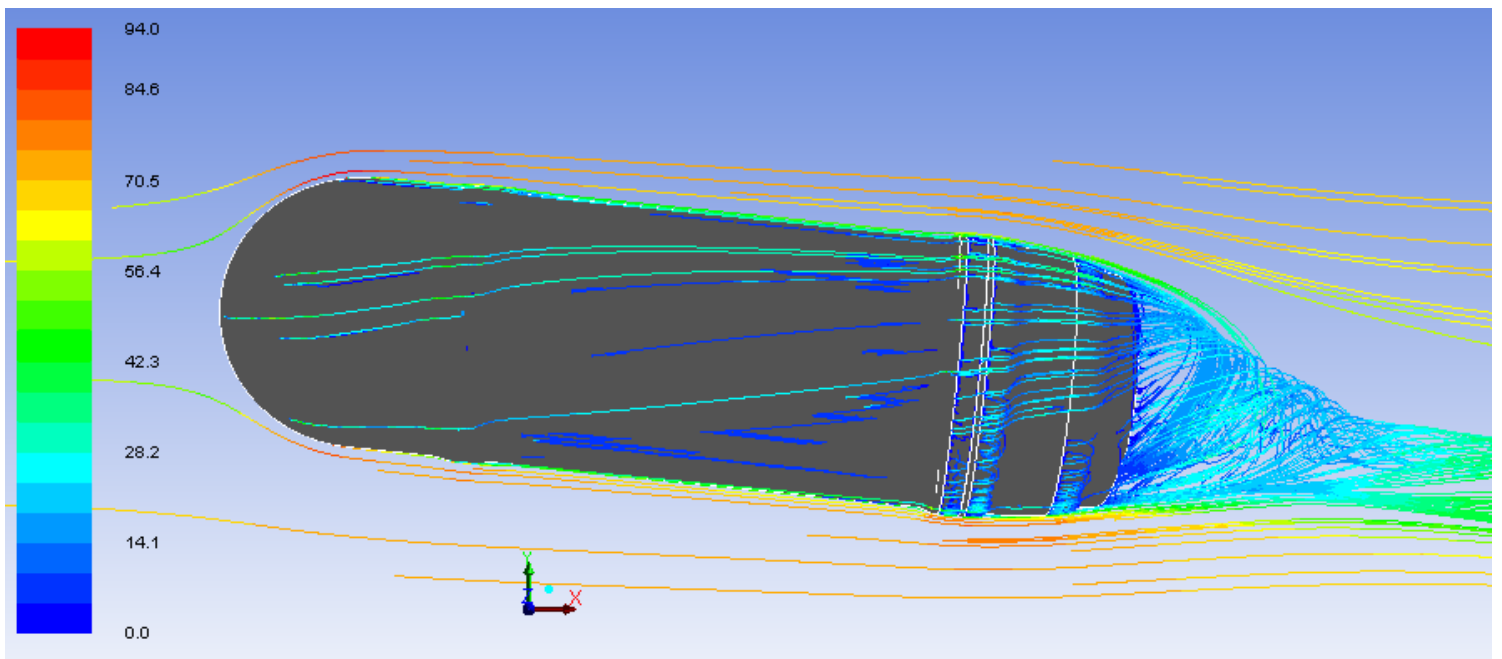

Figure 4-19: Velocity Pathlines Showing Recirculating Flow Aft of $40 \mathrm{~mm}$ Round for $6^{\circ} \mathrm{AOA}$ 


\subsubsection{Tail-deployed Configuration}

It was previously shown that the $40 \mathrm{~mm}$ baseline was gyroscopically stable, but this alone would not provide insight into its dynamic stability. As explained in McCoy (1999), the dynamic stability factor would require additional information including the Magnus moment and pitch damping moment coefficients. To alleviate the possibility of dynamic instability, fins were added near the base of the projectile thereby forcing the $\mathrm{CP}$ aft of the CG. In addition to obtaining aerodynamic lift and drag, the pitching moment coefficient was calculated to verify that the magnitude of $C_{m_{\alpha}}$ was negative. Equation 4-1 was rewritten to account for the negative pitching moment generated due the aerodynamic forces applied at the $\mathrm{CP}$, as shown in Equation 4-2.

$$
C_{m_{C G}}=C_{m_{\text {nose }}}-\left(C_{L} \cos \alpha+C_{D} \sin \alpha\right) \frac{l}{d}
$$

These fixed body CFD simulations were expected to generate more drag on the body due to parasitic drag as well as lift induced drag on the tail fins for higher angles of attack. This was confirmed for $0^{\circ} \mathrm{AOA}$ because the calculated drag coefficient of 0.18 was approximately $13 \%$ greater in magnitude in comparison to the baseline. Figure 4-20 provides the lift and drag at angles of attack ranging from $-10^{\circ}$ to $10^{\circ}$. As shown in Figure 4-21, the data was fitted linearly in which the magnitude of $C_{m_{\alpha}}$ was -0.295 . This value indicated that the $\mathrm{CP}$ was located aft of the CG. That is, a negative pitching moment would be generated for positive angles of attack and a positive pitching moment would be generated for negative angles of attack.

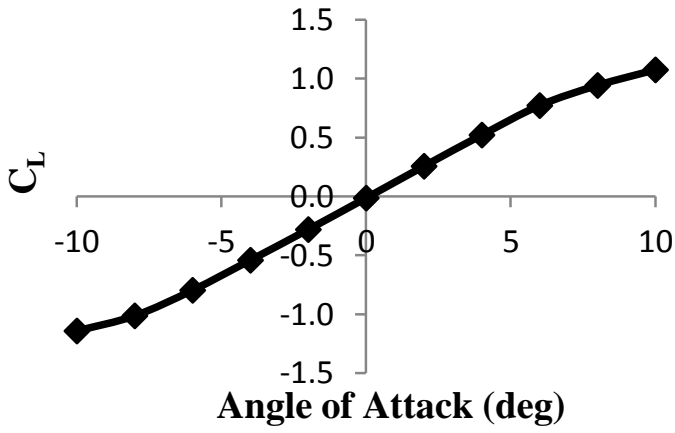

Figure 4-20: CFD Predictions of Aerodynamic Lift and (b) Drag on Tail-deployed Configuration

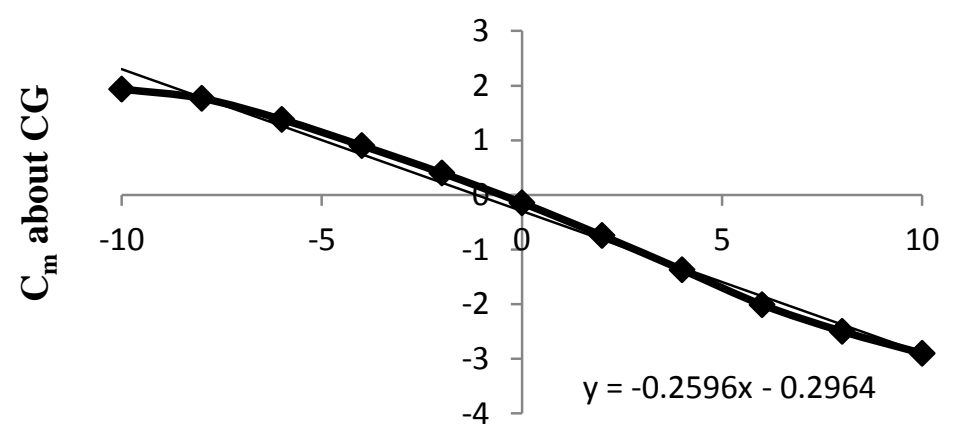

Angle of Attack (deg)

Figure 4-21: Aerodynamic Pitching Moment for Tail-deployed Configuration 
Similar to the $40 \mathrm{~mm}$ baseline round, the results of the simulations for the finned projectile were examined at $0^{\circ}$ and $6^{\circ}$ AOA for a free stream velocity of $70 \mathrm{~m} / \mathrm{s}$. The flow physics were comparable to the $40 \mathrm{~mm}$ round except for the effects due to the tail fins. For $0^{\circ}$ AOA, the maximum velocity occurred once the airflow passed over the hemispherical nose, as highlighted in the red colored contours of Figure 4-22. This was where the largest adverse pressure gradient was calculated, as shown in Figure 4-23. As expected, the maximum pressure coefficient was computed at the stagnation region on the nose tip. Since the pressure contours were axisymmetric, no lift was generated on the body. Figure 4-24 provides the pathlines of velocity magnitude passing around the finned projectile at $0^{\circ} \mathrm{AOA}$. The pathlines clearly show that the flow turned and separated aft of the body. This created two regions of recirculation, similar to the baseline configuration, which was in a region of low pressure. For $6^{\circ} \mathrm{AOA}$, the turning angle was greater on the leeward side causing flow separation to occur and higher flow speed on the windward side due to a smaller turning angle allowing the flow to expand. The flow separation can be clearly shown by displaying the pathlines around the finned projectile at $6^{\circ} \mathrm{AOA}$, as illustrate in Figure 4-25.

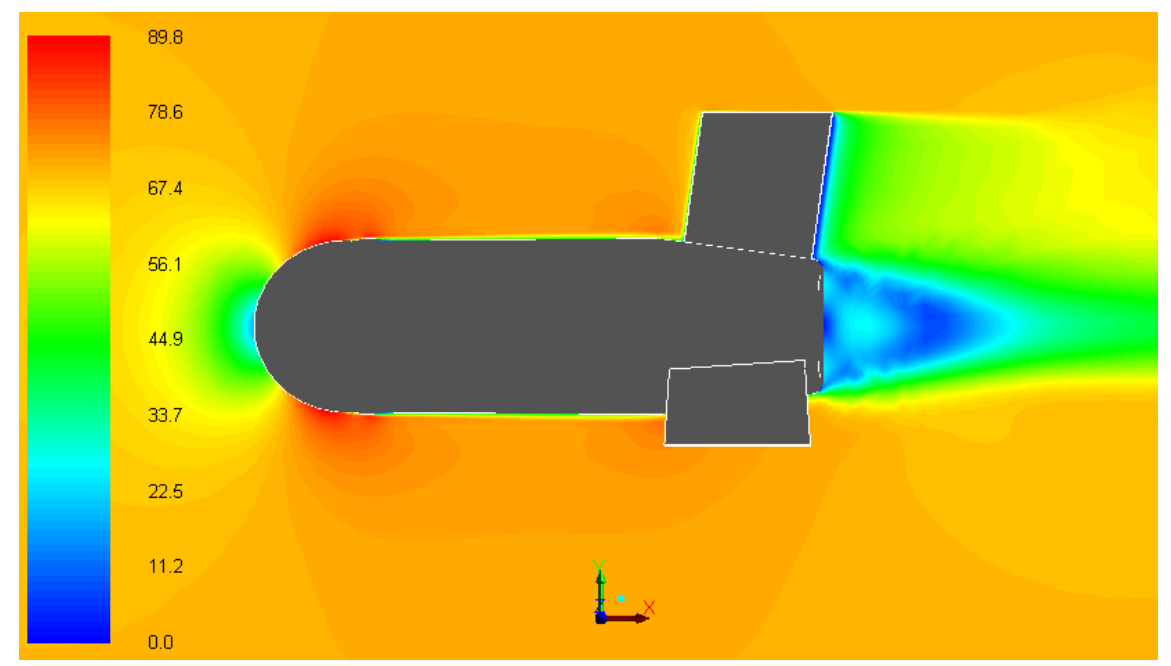

Figure 4-22: Contours of Velocity Magnitude for Tail-deployed Configuration at $0^{\circ} \mathrm{AOA}$

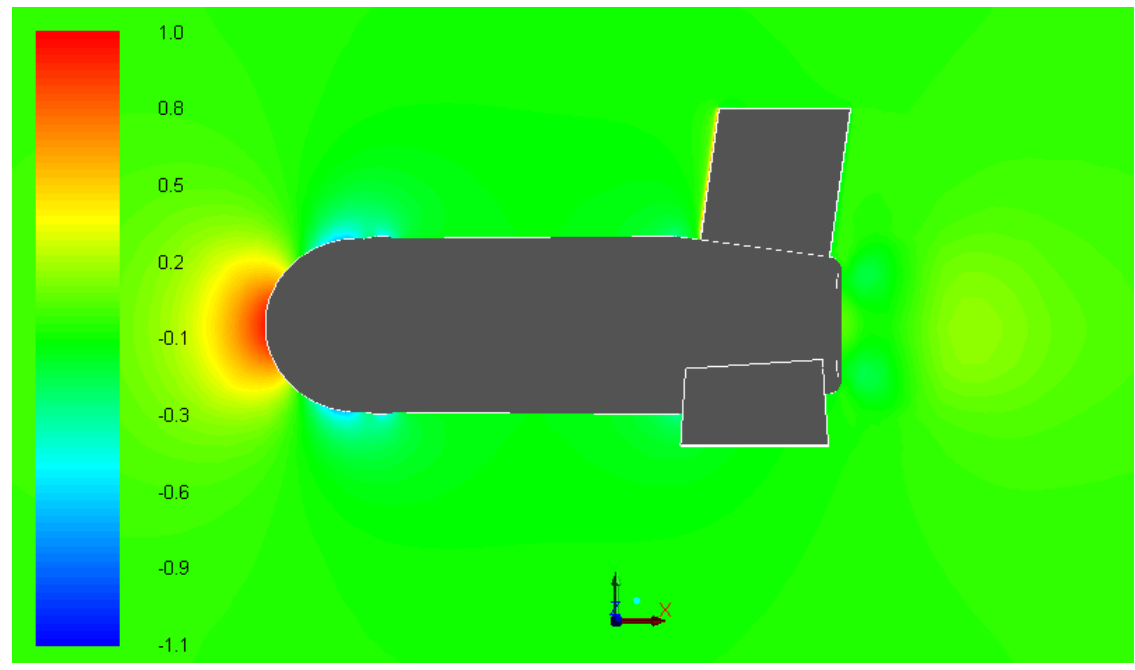

Figure 4-23: Contours of Pressure Coefficient for Tail-deployed Configuration at $0^{\circ} \mathrm{AOA}$ 


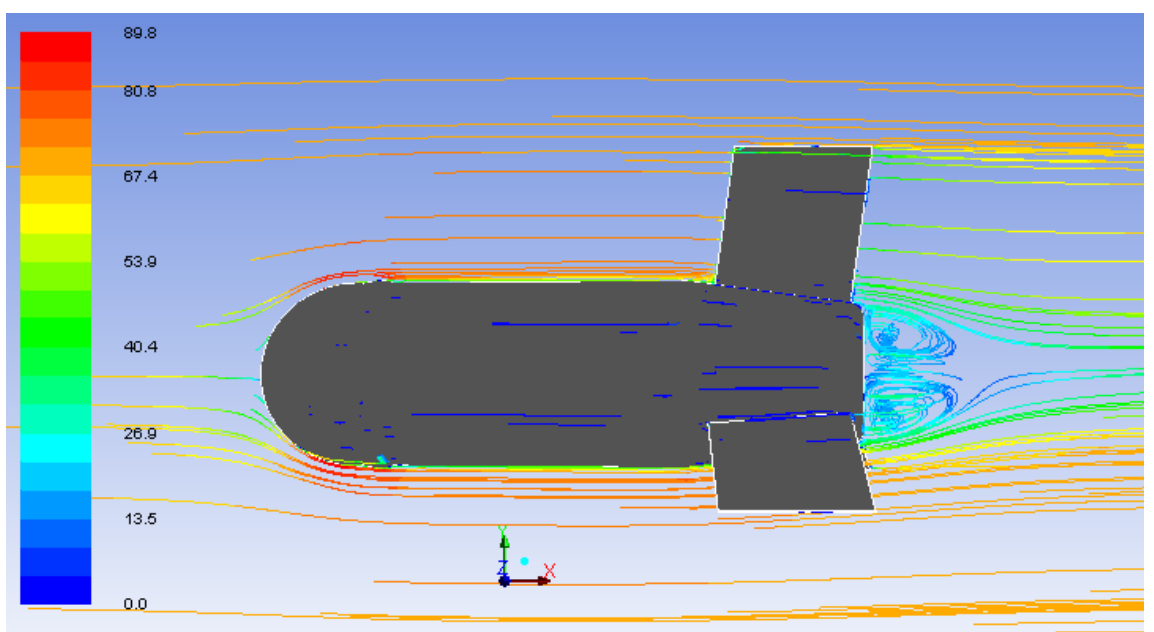

Figure 4-24: Velocity Pathlines Showing Recirculation Regions Aft of Tail-deployed Configuration at $0^{\circ}$ AOA

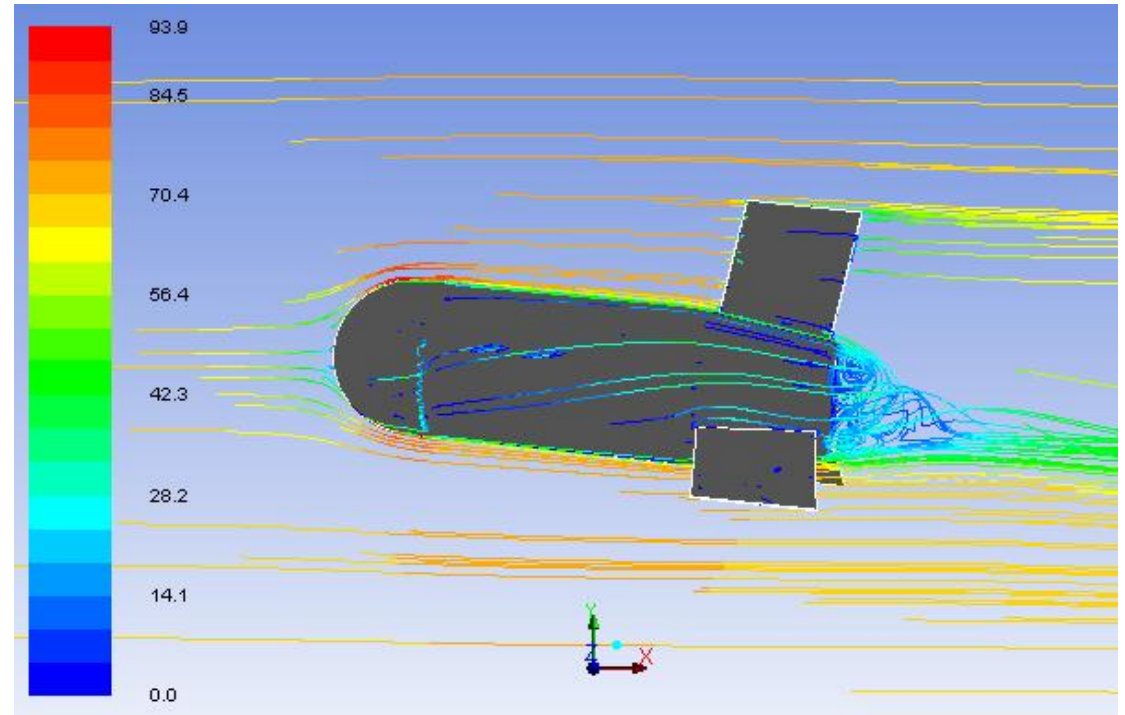

Figure 4-25: Velocity Pathlines Showing Recirculation Regions Aft of Tail-deployed Configuration at $6^{\circ}$ AOA

\subsubsection{Fully Deployed MAV Configuration}

Results from the fixed body CFD simulations were also analyzed for the fully deployed MAV configuration at a free stream velocity of $45 \mathrm{~m} / \mathrm{s}$. These simulations were performed for no tail deflection. In comparison to the results for the fin-stabilized projectile, the lift and drag was considerably larger in magnitude due to the additional lifting surfaces. The simulations showed that for an angle of attack of $0^{\circ}$, the drag coefficient increased by approximately $42 \%$ with a magnitude of 0.255 . The increased drag was attributed to the additional parasitic drag and lift induced drag on the wing for increasing angles of attack. It was determined that the MAV was optimized for a body angle of attack of $6^{\circ}$ giving a maximum lift-todrag of approximately 4.5. Figure 4-26 provides the aerodynamic drag and lift-to-drag ratio for angles of attack ranging from $0^{\circ}$ to $10^{\circ}$. The drag appeared to increase considerably for a mild change in the angle of attack. As previously explained, the computed drag on the baseline round was over-predicted according to the measured drag. Therefore, the computed drag on the wings-deployed configuration was expected to be over-predicted as well for increasing angles of attack. 

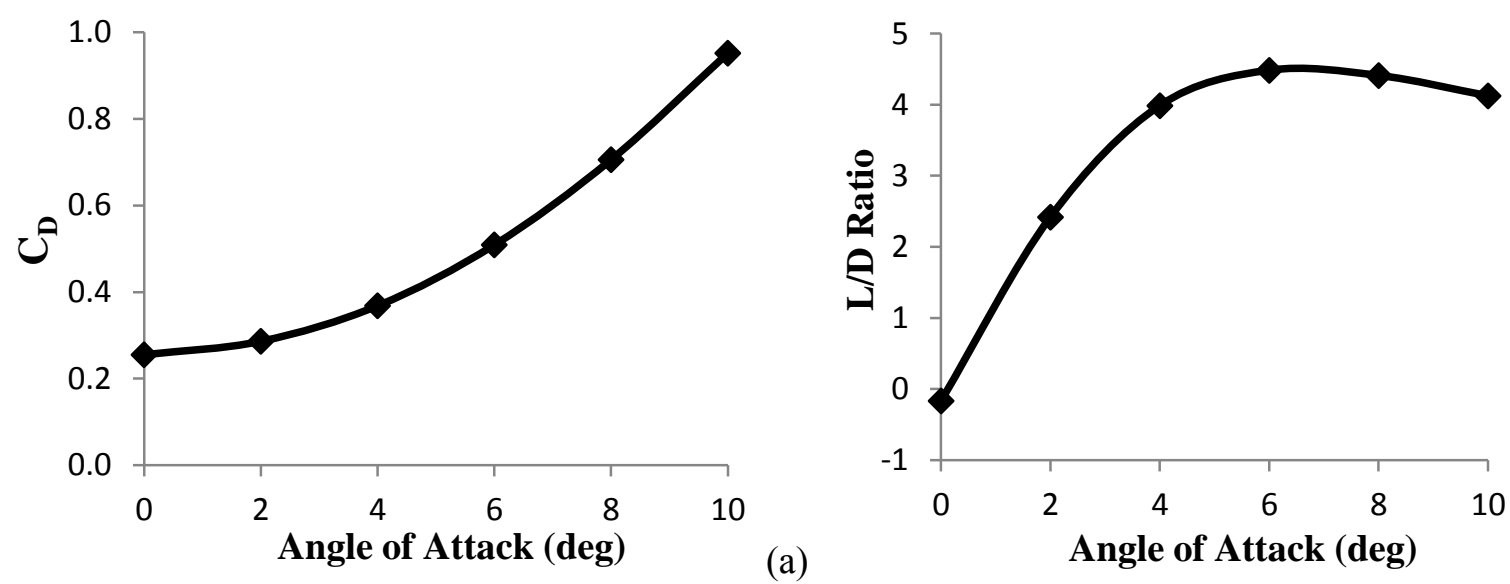

(b)

Figure 4-26: CFD Predicted (a) Aerodynamic Drag and (b) Lift-to-Drag for Inverted Y-tail Design

In addition to analyzing the aerodynamic forces, the flow physics were analyzed as well. Figure 4-27 provides the velocity contours in which the maximum velocity of $57 \mathrm{~m} / \mathrm{s}$ occurred as the airflow passed over the hemispherical nose. Similar to the other two configurations, the free stream velocity was subsonic everywhere in the flow. Therefore, the airspeed was reduced upstream from the nose since the fluid was anticipating an obstruction in the airflow. At the location of maximum velocity, an adverse pressure gradient was present, as shown in Figure 4-28. At $0^{\circ}$ AOA, the pressure contours were nearly axisymmetric and consequently, the amount of lift generated on the body was minimal. The flow characteristics aft of the MAV's body were similar to the fin-stabilized projectile. That is, at $0^{\circ} \mathrm{AOA}$, the turning angle at the aft end of the body was quite large and caused two regions of recirculation, as shown in Figure 4-29. As the body angle of attack increased, the turning angle was greater on the leeward side than on the windward side. In comparison to the previous two configurations, recirculating airflow was generated on the leeward side while the airflow on the windward side increased, as illustrated in Figure 4-30. Additionally, an asymmetry existed in the pressure contours in which higher pressure on the under surface and lower pressure on the upper surface produced lift on the body.

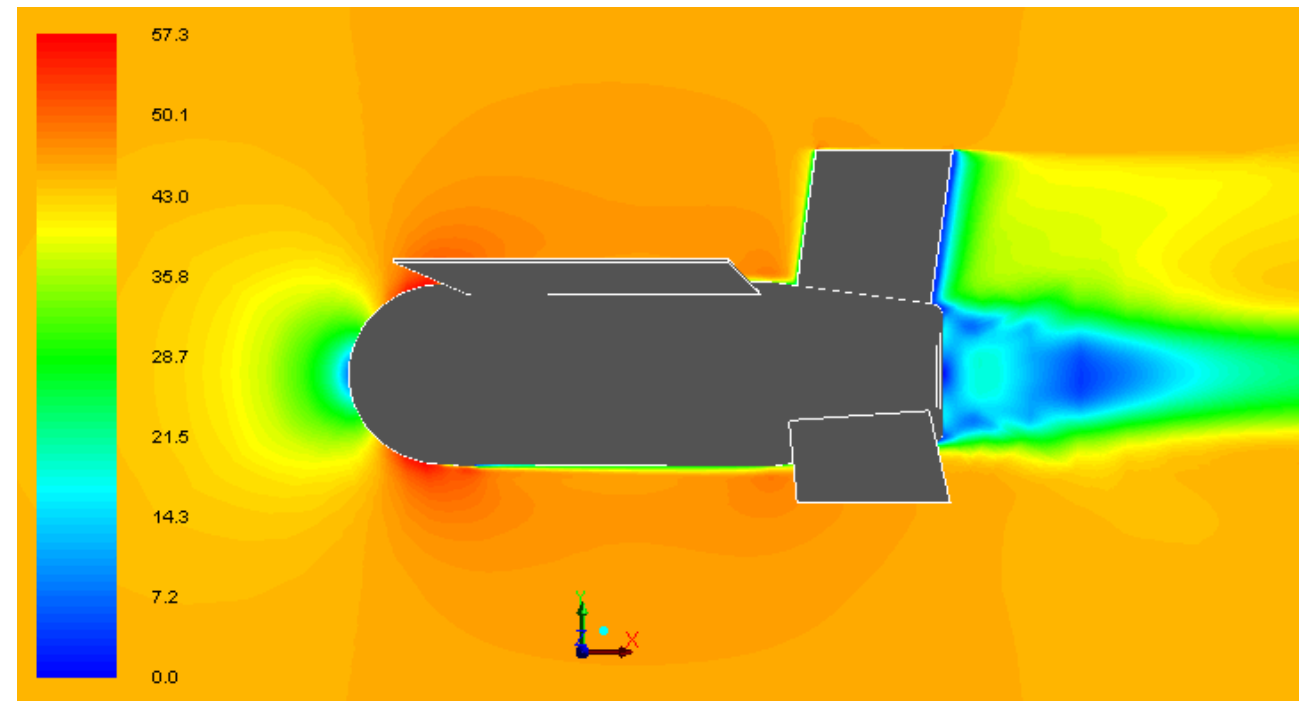

Figure 4-27: Contours of Velocity Magnitude for Inverted Y-tail Configuration at $0^{\circ} \mathrm{AOA}$ 


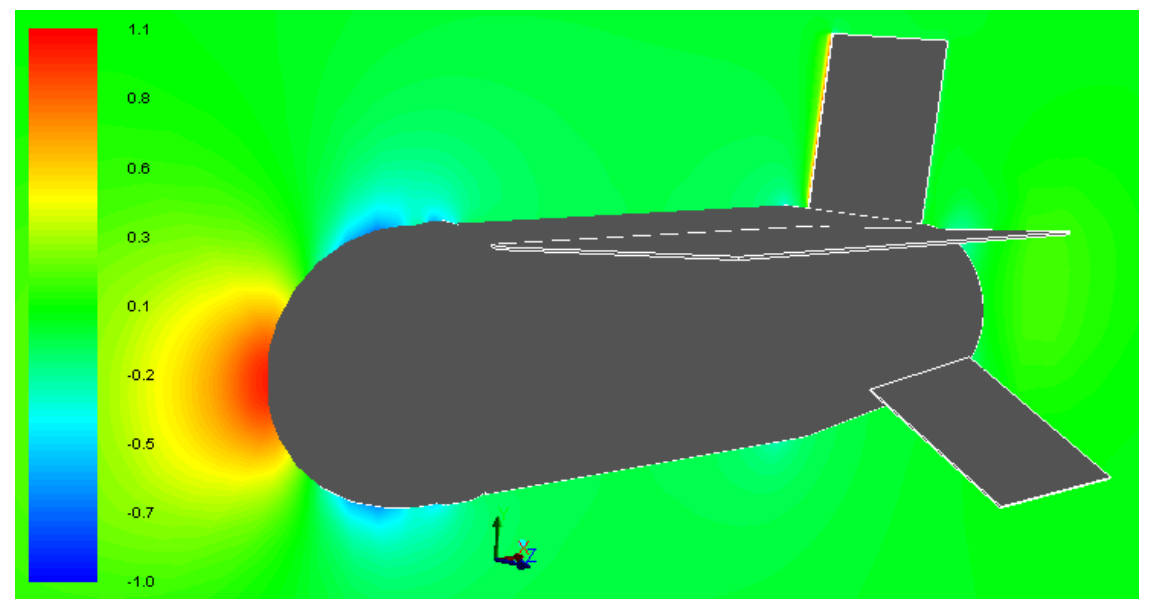

Figure 4-28: Contours of Pressure Coefficient for Inverted-Y Tail Configuration at $0^{\circ} \mathrm{AOA}$

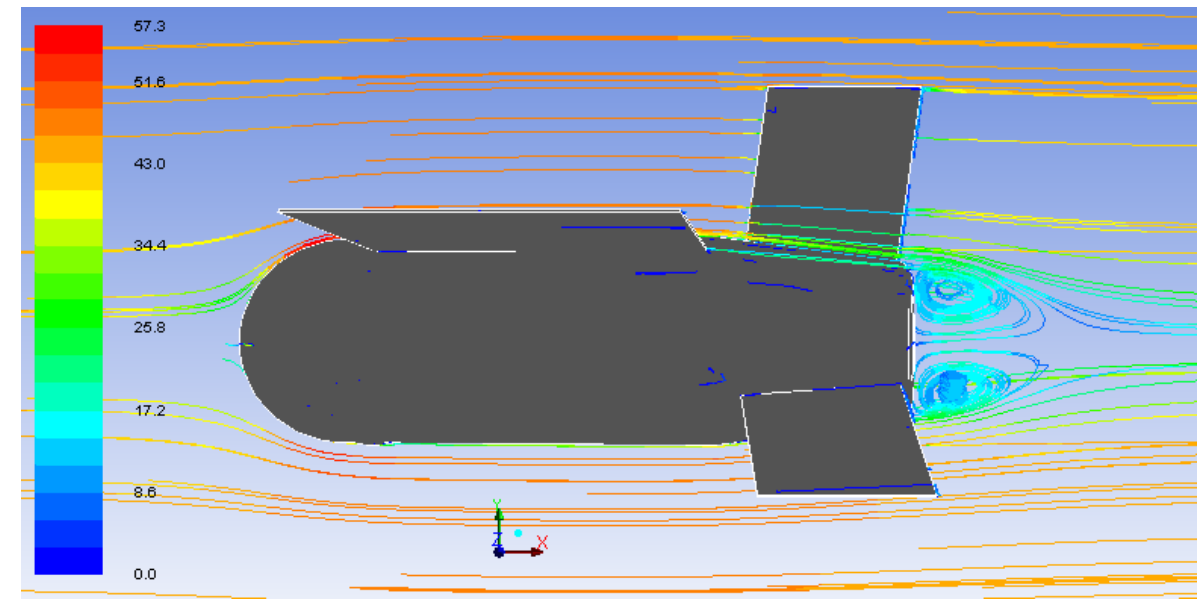

Figure 4-29: Pathlines of Velocity Magnitude Showing Recirculation behind Inverted Y-tail of Fully Deployed MAV Configuration at $0^{\circ} \mathrm{AOA}$

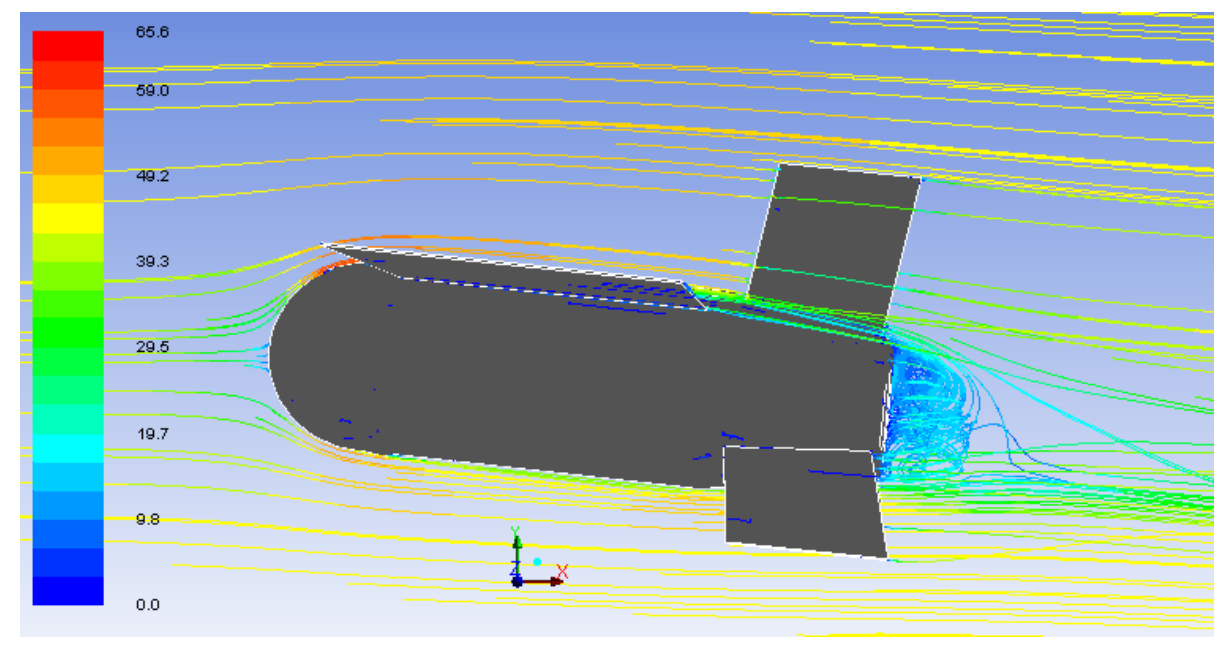

Figure 4-30: Pathlines of Velocity Magnitude Showing Recirculation behind Inverted Y-tail of Fully Deployed MAV Configuration at $6^{\circ} \mathrm{AOA}$ 
Once it was determined that the MAV was optimized for a body angle of attack of $6^{\circ}$, the two fins forming the inverted V-tail were deflected in order to achieve moment equilibrium about the CG. It should be noted that the tail design was such that the fins pivoted about its leading edge in which positive tail deflection corresponded to the trailing edge deflected upwards. Figure 4-31 provides the magnitude of the moment coefficient about the CG for different tail deflection angles. It was determined that a $5^{\circ}$ tail deflection with the CG located at $39.5 \mathrm{~mm}$ aft of the nose was necessary to achieve moment equilibrium.

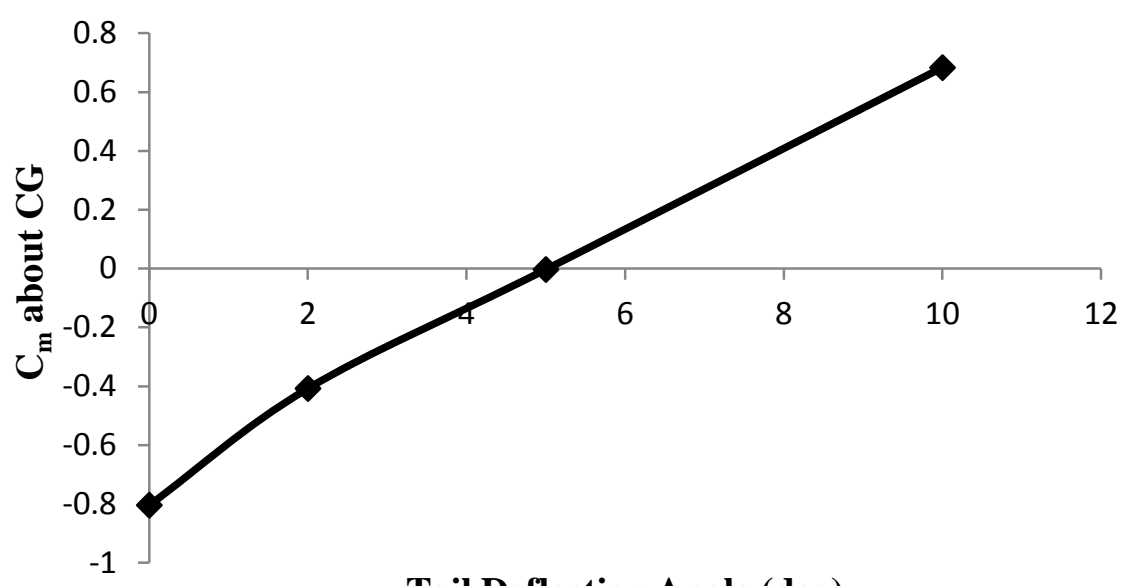

Tail Deflection Angle (deg)

Figure 4-31: Moment Coefficient about CG of Inverted Y-tail with Variation in Tail Deflection Angle

Another CFD study was performed on the Y-tail configuration. As shown in Figure 4-32, the MAV was optimized for a body angle of attack of $7^{\circ}$. Since the ratio of lift-to-drag did not differ significantly for a body angle of attack of $6^{\circ}$, this angle was selected for direct comparison to the inverted Y-tail configuration. A front view of the MAV at a $6^{\circ}$ AOA highlighted the airflow traveling over the wing as shown in Figure 4-33. Once the Y-tail design was optimized, the fins forming the V-tail were deflected at different angles to determine what tail deflection would be required to produce moment equilibrium at the CG location. This was achieved by deflecting the fins by $7.5^{\circ}$, as shown in Figure 4-34.

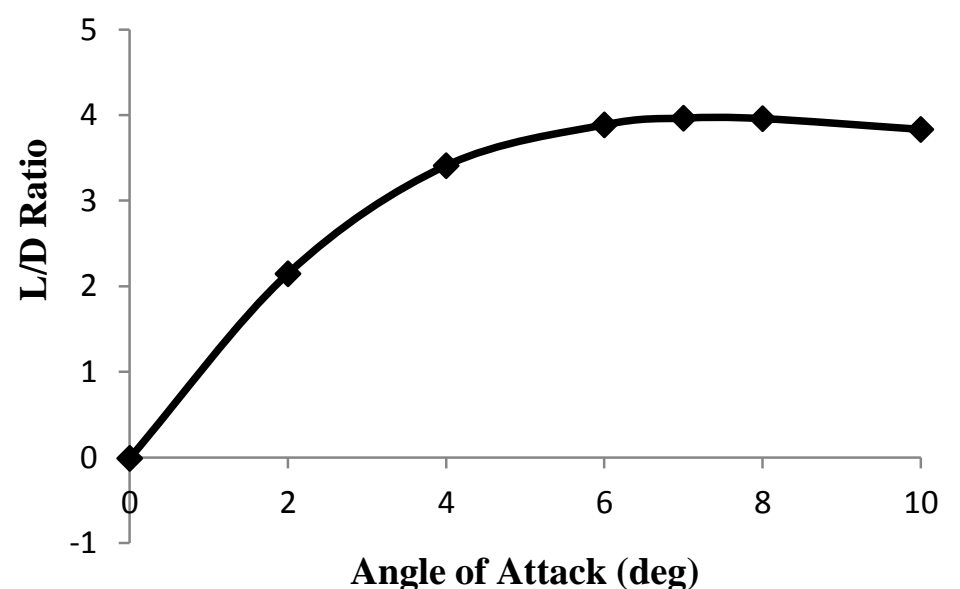

Figure 4-32: Lift-to-Drag Ratio for Y-tail Design of Fully Deployed MAV Configuration Showing Body Optimized at $6^{\circ} \mathrm{AOA}$ 


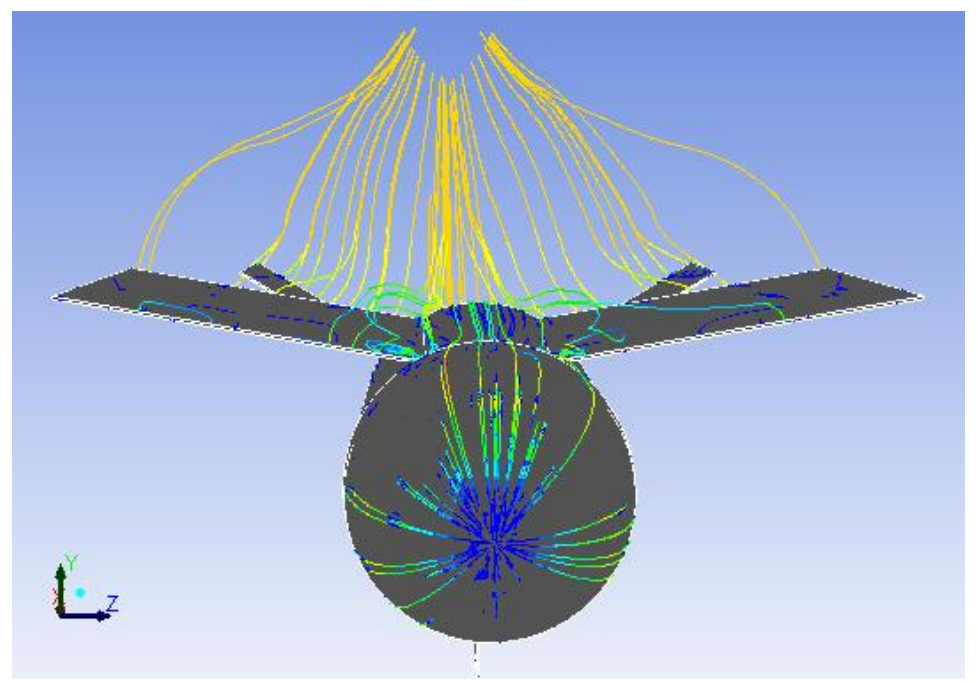

Figure 4-33: Pathlines of Velocity Magnitude Passing over Y-tail Configuration at $6^{\circ} \mathrm{AOA}$

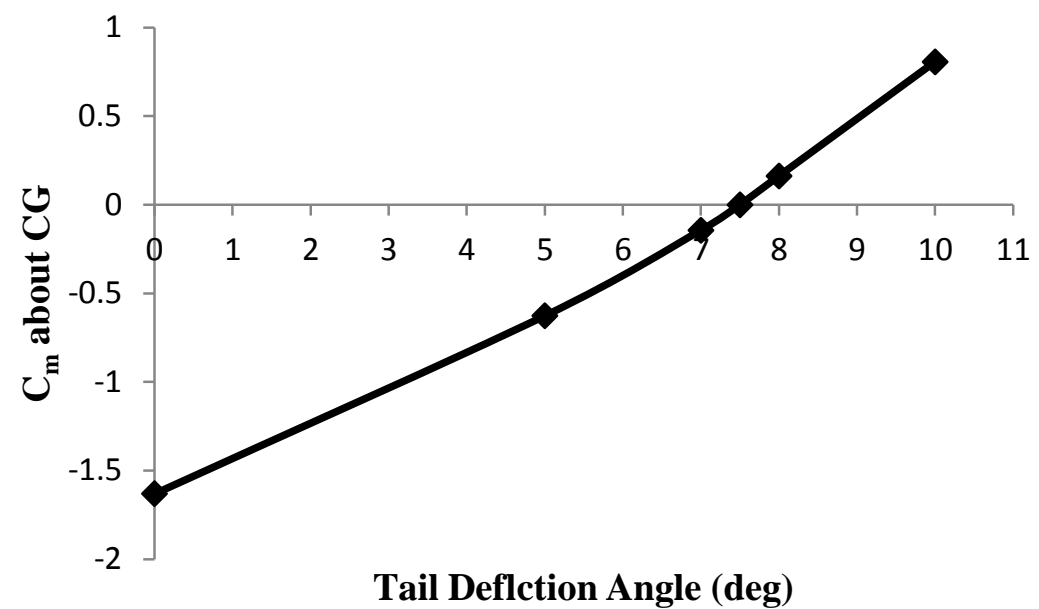

Figure 4-34: Moment Coefficient about CG for Y-tail Design with Variation in Tail Deflection Angle 


\section{Chapter 5 Initialization Requirements for Numerical Flight Testing}

\subsection{Grid Design and Boundary Conditions}

The design of the hybrid mesh for simulating free body motion were generated by following a similar procedure for conducting traditional CFD simulations. The dissimilarity was that the outer fluid domain was considerably larger in volume. In terms of distance, the dimensions of the unstructured mesh consisted of a range of $300 \mathrm{~m}$, a height of $150 \mathrm{~m}$, and width of $50 \mathrm{~m}$. The nodal count of the unstructured mesh did not increase the overall nodal count significantly; that is, the nodal count did not exceed two million nodes. The size of the farfield for the baseline and tail-deployed configurations was created for simulating their ballistic trajectory to apex. Similarly, the wings-deployed configuration had identical dimensions for simulating the MAV gliding to its destination beginning at the apex of the original ballistic trajectory. Because the transformable MAV would be launched through a stagnant fluid, like a real-life fired projectile, the velocity inlet and pressure outlet boundary conditions were not required. Figure 5-1 shows the complete hybrid mesh for the fin-stabilized projectile simulation. At the far right corner, the cells were considerably more dense, which was the initial location of the finned projectile. The desired angle of launch was $45^{\circ}$ and therefore, the unstructured mesh was reoriented counterclockwise, as shown in Figure 5-1. This step was taken to avoid having to specify initial position and initial translational and rotational velocity components. However, this did require components of gravitational acceleration, $g_{x}$ and $g_{y}$, to be specified. Since the wings-deployed configuration was initialized at the apex of the trajectory and the body would be parallel with the ground, only the vertical component of gravitational acceleration was necessary. Figure 5-2 provides the hybrid mesh that was generated for the wings-deployed configuration.

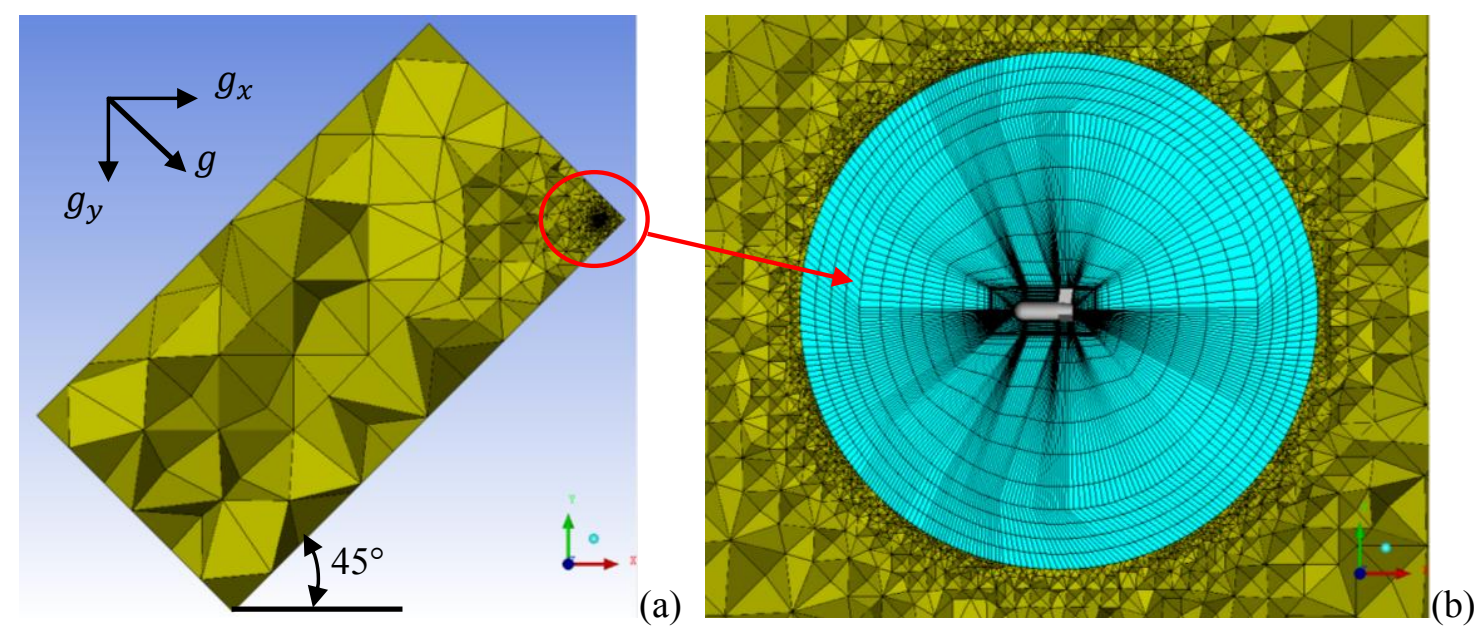

Figure 5-1: Hybrid Mesh Showing (a) Farfield and Outer Fluid Domain for Simulating Free Body Motion of Tail-deployed Configuration and (b) Inner Fluid Domain Magnified to Show Unstructured Cells Growing Outward from Non-conformal Interface 


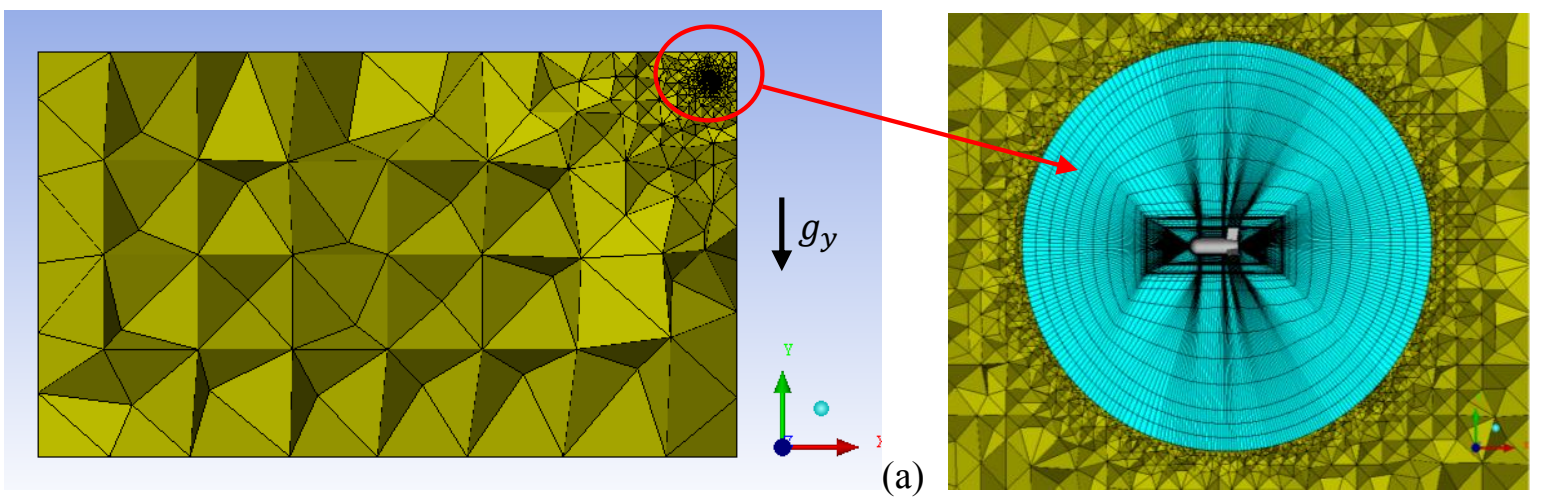

Figure 5-2: Hybrid Mesh Showing (a) Farfield and Outer Fluid Domain for Simulating Free Body Motion of Fully Deployed MAV Configuration and (b) Inner Fluid Domain Magnified to Show Unstructured Cells Growing Outward from Non-conformal Interface

\subsection{Solver}

The solution methodology for the pressure-velocity solver was configured identically to the fixed body CFD analyses. That is, the SIMPLEC scheme was employed. Also, the Green-Gauss cell-based discretization method was selected over the node-based method to reduce the amount of computational time as much as possible. For the entire simulation, the solution was time-dependent. This was necessary since the structured mesh would dynamically move through the unstructured mesh upon every time step. Initially, the time step was set to $2 \times 10^{-5} \mathrm{sec}$; however, this was successfully increased to $1 \times 10^{-4} \mathrm{sec}$. The simulations showed that increasing the time step any further would lead to cell quality issues within the unstructured mesh in which the cells would collapse on themselves causing the solution to become unstable.

The reference values were identical for the various configurations except for the velocity, as provided in Table 5-1. The reference velocity for the baseline and tail-deployed configurations was set to $70 \mathrm{~m} / \mathrm{s}$, whereas the reference velocity for the fully deployed MAV was set to $45 \mathrm{~m} / \mathrm{s}$. Also, the hybrid mesh had to be initialized before executing the simulations, in which the parameters and their values are provided in Table 5-2. After running a few trial simulations, it was determined that the initial turbulent kinetic energy and specific dissipation rate required a small value in order to avoid having an unstable solution. This was a necessary requirement because the airflow at a far distance from the aerodynamic body was calm and therefore the turbulent kinetic energy and specific dissipation rate were negligible.

Before activating the 6-DOF solver to simulate free body motion, the baseline and tail-deployed configurations were fixed to translate along the negative x-axis at $70 \mathrm{~m} / \mathrm{s}$ and rotate clockwise viewed from the base of the projectile at $3600 \mathrm{RPM}$ or $377 \mathrm{rad} / \mathrm{s}$. For the fully deployed MAV configuration, there was initially only translation with a velocity of $45 \mathrm{~m} / \mathrm{s}$. The rationale behind performing these initial simulations was to allow a boundary layer to be developed around the aerodynamic body as it traveled through still air. A user-defined function (UDF) was compiled and loaded into the solver to carry out these simulations in which a macro was utilized for defining the motion at the CG of the aerodynamic body. The source code for this UDF can be found in Appendix A. This simulation was first conducted by configuring the spatial discretization scheme such that the pressure was set to standard and the momentum, turbulent kinetic energy, and specific dissipation rate were set to first order upwind. This provided the solution with an initial guess before switching the discretization scheme to second order upwind for a more accurate solution. For the initial simulation, the number of time steps for the first order accurate solution was executed for approximately 25 time steps and the second order solution was executed for approximately 125 time steps. Following this simulation was the free body motion analysis 
using the 6-DOF solver. The UDF for performing the numerical flight testing of the aerodynamic bodies utilized another macro for defining the mass and moments of inertia properties, which can also be found in Appendix A. The procedure for compiling the UDFs and configuring the 6-DOF solver can be found in Appendix B.

Table 5-1: Reference Values for Numerical Flight Testing

\begin{tabular}{|c|c|c|c|}
\hline Parameter & $\begin{array}{c}\text { Baseline and Tail-Deployed } \\
\text { Configuration }\end{array}$ & $\begin{array}{c}\text { Wings-Deployed MAV } \\
\text { Configuration }\end{array}$ & Units \\
\hline Area & 0.00126 & 0.00126 & $\mathrm{~m}^{2}$ \\
\hline Density & 1.225 & 1.225 & $\mathrm{~kg} / \mathrm{m}^{3}$ \\
\hline Length & 0.04006 & 0.04006 & $\mathrm{~m}$ \\
\hline Pressure & 0 & 0 & $\mathrm{~Pa}$ \\
\hline Temperature & 288.16 & 288.16 & $\mathrm{~K}$ \\
\hline Velocity & 70 & 45 & $\mathrm{~m} / \mathrm{s}$ \\
\hline Viscosity & $1.79 \mathrm{E}-05$ & $1.79 \mathrm{E}-05$ & $\mathrm{~kg} /(\mathrm{m}-\mathrm{s})$ \\
\hline
\end{tabular}

Table 5-2: Initialization Parameters for Numerical Flight Testing

\begin{tabular}{|c|c|c|}
\hline Parameter & Magnitude & Units \\
\hline Pressure & 0 & $\mathrm{~Pa}$ \\
\hline X-velocity & 0 & $\mathrm{~m} / \mathrm{s}$ \\
\hline Y-velocity & 0 & $\mathrm{~m} / \mathrm{s}$ \\
\hline Z-velocity & 0 & $\mathrm{~m} / \mathrm{s}$ \\
\hline Turbulent Kinetic Energy & 0 & $\mathrm{~m}^{2} / \mathrm{s}^{2}$ \\
\hline Specific Dissipation Rate & 0 & $1 / \mathrm{s}$ \\
\hline
\end{tabular}




\section{Chapter 6 Numerical Flight Testing}

The following sections will discuss the results of free flight motion using a relatively new tool that couples a CFD analysis with a 6-DOF rigid body flight dynamics model. These simulations are what this study has referred to as numerical flight testing. The flight characteristics were predicted for three different configurations, which included the baseline, tail-deployed, and wings-deployed models. Information for the moments of inertia was required for each of the configurations, as provided in Table 6-1. These properties were taken directly from the SolidWorks models at the location of the CG.

Table 6-1: Moments and Products of Inertia Properties for Baseline, Tail-deployed, and Wings-Deployed Configurations Experiencing Free Body Motion

\begin{tabular}{|c|c|c|c|c|c|}
\hline \multirow{2}{*}{$\begin{array}{c}\text { Moments and } \\
\text { Products of Inertia }\end{array}$} & \multirow{2}{*}{$\begin{array}{c}\text { Baseline } \\
\text { Configuration }\end{array}$} & \multirow{2}{*}{$\begin{array}{c}\text { Tail-deployed } \\
\text { Configuration }\end{array}$} & \multicolumn{2}{|c|}{ Wings-deployed Configuration } & \multirow{2}{*}{ Units } \\
\cline { 4 - 6 } & $3.4385 \mathrm{E}-05$ & $3.4832 \mathrm{E}-05$ & $2.4381 \mathrm{E}-04$ & $2.4391 \mathrm{E}-04$ & $\mathrm{~kg}-\mathrm{m}^{2}$ \\
\hline $\mathrm{I}_{\mathrm{xx}}$ & $3.9673 \mathrm{E}-04$ & $2.3284 \mathrm{E}-04$ & $1.1930 \mathrm{E}-03$ & $1.1153 \mathrm{E}-03$ & $\mathrm{~kg}-\mathrm{m}^{2}$ \\
\hline $\mathrm{I}_{\mathrm{yy}}$ & $3.9673 \mathrm{E}-04$ & $2.3284 \mathrm{E}-04$ & $1.1833 \mathrm{E}-03$ & $1.1252 \mathrm{E}-03$ & $\mathrm{~kg}-\mathrm{m}^{2}$ \\
\hline $\mathrm{I}_{\mathrm{zz}}$ & 0 & 0 & $1.1068 \mathrm{E}-05$ & $1.0715 \mathrm{E}-05$ & $\mathrm{~kg}-\mathrm{m}^{2}$ \\
\hline $\mathrm{I}_{\mathrm{xy}}$ & 0 & 0 & $9.8680 \mathrm{E}-08$ & $-2.3060 \mathrm{E}-07$ & $\mathrm{~kg}-\mathrm{m}^{2}$ \\
\hline $\mathrm{I}_{\mathrm{xz}}$ & 0 & 0 & $-1.3630 \mathrm{E}-08$ & $-3.6280 \mathrm{E}-08$ & $\mathrm{~kg}-\mathrm{m}^{2}$ \\
\hline $\mathrm{I}_{\mathrm{yz}}$ & & &
\end{tabular}

\subsection{Baseline Configuration}

\subsubsection{Sensitivity Analysis}

A sensitivity analysis was initially performed on the baseline configuration in which two simulations were conducted for approximately 120 time steps at 70 iterations per time step and again at 15 iterations per time step. The results of the drag coefficient were compared for the two simulations and plotted as a percent difference, as shown below in Figure 6-1. This data clearly showed that the drag did not differ by more than $1.5 \%$ and therefore, the numerical flight test was executed at 15 iterations per time step to reduce the total computational time of this simulation.

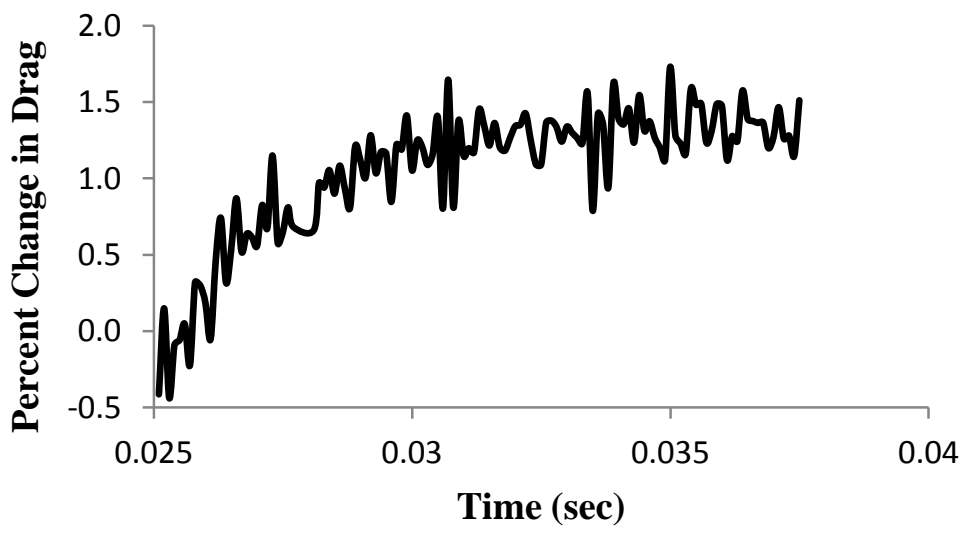

Figure 6-1: Sensitivity Analysis on Baseline Configuration for Numerical Flight Testing 


\subsubsection{Stability Characteristics}

The results of the aerodynamic behavior for the baseline round undergoing free body motion were analyzed for a total simulation time of $0.35 \mathrm{sec}$. Initially, the simulation required approximately 7 days or 875 CPU-hours to obtain the computational results based on the sensitivity analysis presented in the previous section. Because of the dynamic instability issues encountered in the numerical flight testing of the baseline round, additional simulations were executed in attempts to resolve these issues.

The next approach was to simplify the geometry by removing the obturator groove and backward facing steps, but the dynamic instability remained an issue. Upon recommendations from ANSYS, the number of iterations was increased to 100 iterations per time step to achieve improved convergence of the residuals. This was a more computationally expensive simulation that required 10 days or $500 \mathrm{CPU}$-hours of run time for a shorter flight time of approximately $0.2 \mathrm{sec}$. However, the improved convergence still predicted dynamic instability in the solution. ANSYS further recommended to refine the mesh to capture more of the flow physics. These suggestions appeared to improve the stability of the projectile, but the results were unrealistically over-predicted. These over-predictions can be clearly shown in the $\alpha-\beta$ curve of Figure 6-2 in which the projectile appeared to reach a maximum pitching and yawing motion of approximately $40^{\circ}$. It should be noted that the baseline round was expected to have a small dynamic instability causing an in-flight conical motion similar to the dynamic behavior provided in Figure 2-24. Additionally, this in-flight conical motion experienced in $40 \mathrm{~mm}$ rounds was confirmed in a private conversation with Chemring Ordnance.

Another technique recommended by ANSYS was to run the coupled CFD/6-DOF simulation using the in-viscid model, instead. Again, this approach still generated similar dynamic instability in the $40 \mathrm{~mm}$ round as before. A final method in attempts to solve this dynamic instability was considered in which the time step was selected based on the Courant number using Equation 6-1. The time step was evaluated by finding the average Courant number on the surface of the projectile as well as the average grid spacing on the surface of the projectile. Assuming the velocity was equal to the free stream velocity of $70 \mathrm{~m} / \mathrm{s}$, the time step was determined to be $5 \times 10^{-5} \mathrm{sec}$, which is half of the original time step. The simulation was executed at $0.1 \mathrm{sec}$ into the previous numerical flight test and was performed for approximately $0.06 \mathrm{sec}$. A comparison is provided in Figure 6-2 that clearly shows that by reducing the time step by half, the dynamic instability in the $40 \mathrm{~mm}$ round is still present. However, this instability progressed more slowly.
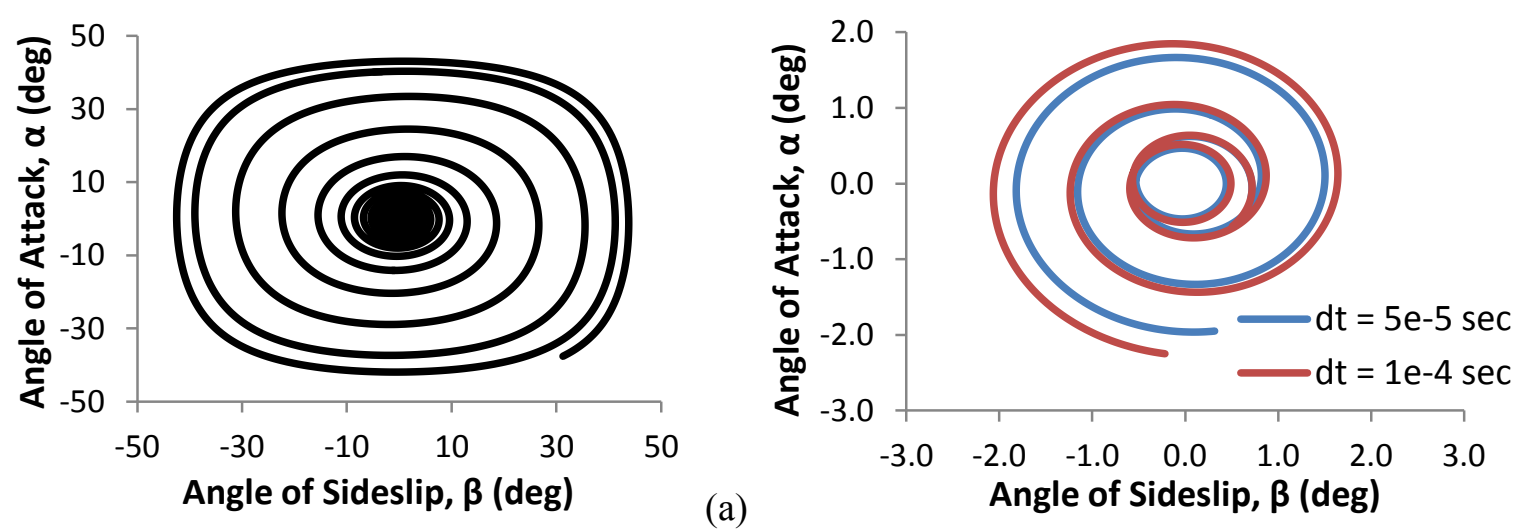

Figure 6-2: Numerical Flight Test Results of $40 \mathrm{~mm}$ Baseline Round (a) $\alpha-\beta$ Curve for 0.35 sec and (b) $\alpha-\beta$ Curve Comparison for Two Different Time Steps

$$
C=\frac{V \Delta t}{\Delta x}
$$


The contours of velocity magnitude are presented in Figure 6-3 at $0.1 \mathrm{sec}$ after being launched from the weapon system. The maximum velocity occurred in the wake of the projectile having a magnitude of $94 \mathrm{~m} / \mathrm{s}$. Similar to the traditional CFD analysis, the airflow remained subsonic everywhere in the flow and therefore, the still air anticipated the moving projectile causing the relative airspeed to be reduced upstream from the hemispherical nose.

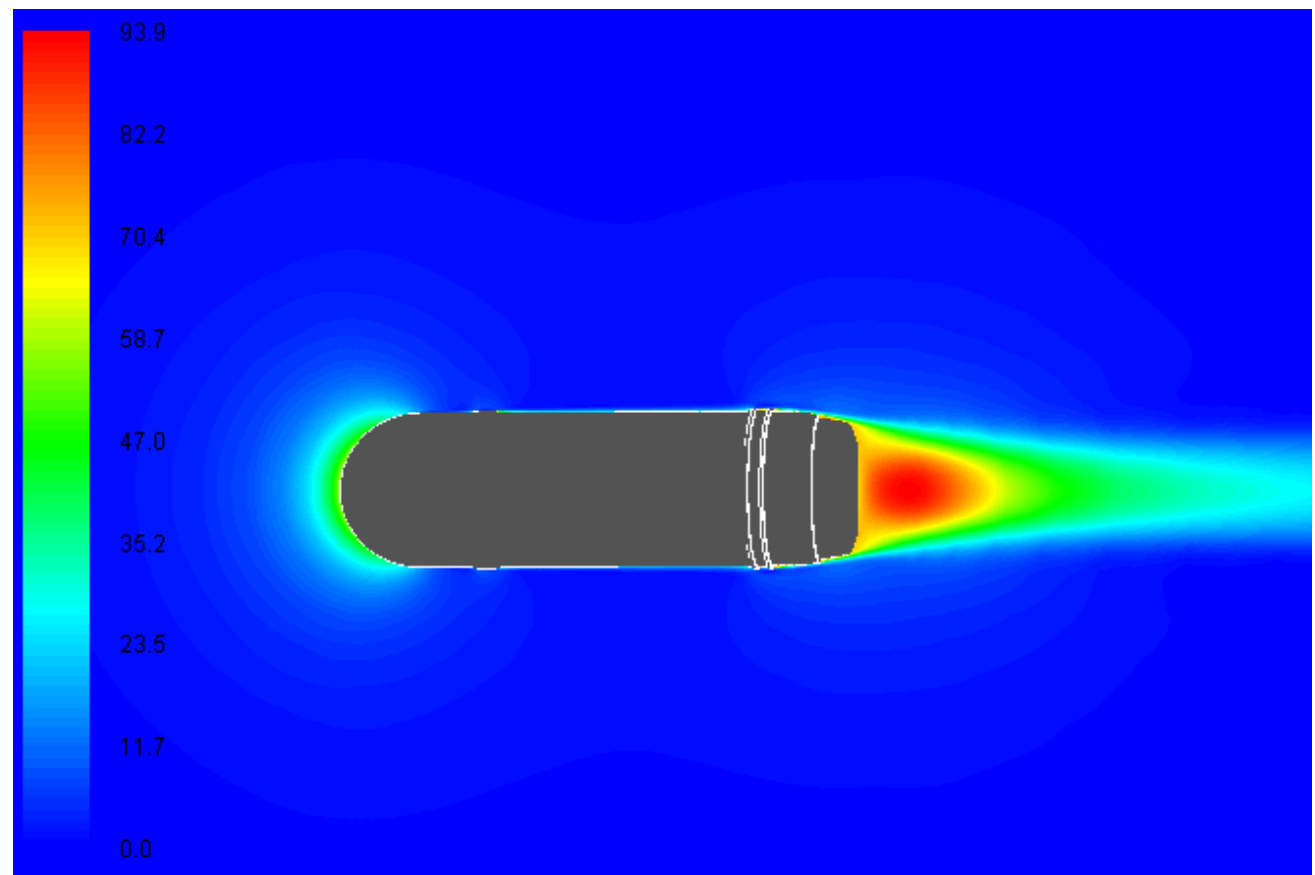

Figure 6-3: Velocity Contours of $40 \mathrm{~mm}$ Baseline Configuration at 0.1 Second after Launch

\subsubsection{Aerodynamic Force Characteristics}

The free flight aerodynamic lift and drag compared well with the traditional CFD analysis from launch to 0.2 second. That is, negligible lift was generated on the symmetric body since the angle of attack was approximately $0^{\circ}$. Also, the drag compared well with a coefficient magnitude of 0.159 . In the onset of dynamic instability, both lift and drag began fluctuating rapidly, as shown in Figure 6-4. At this point in the simulation, the results for aerodynamic lift and drag were unreliable.
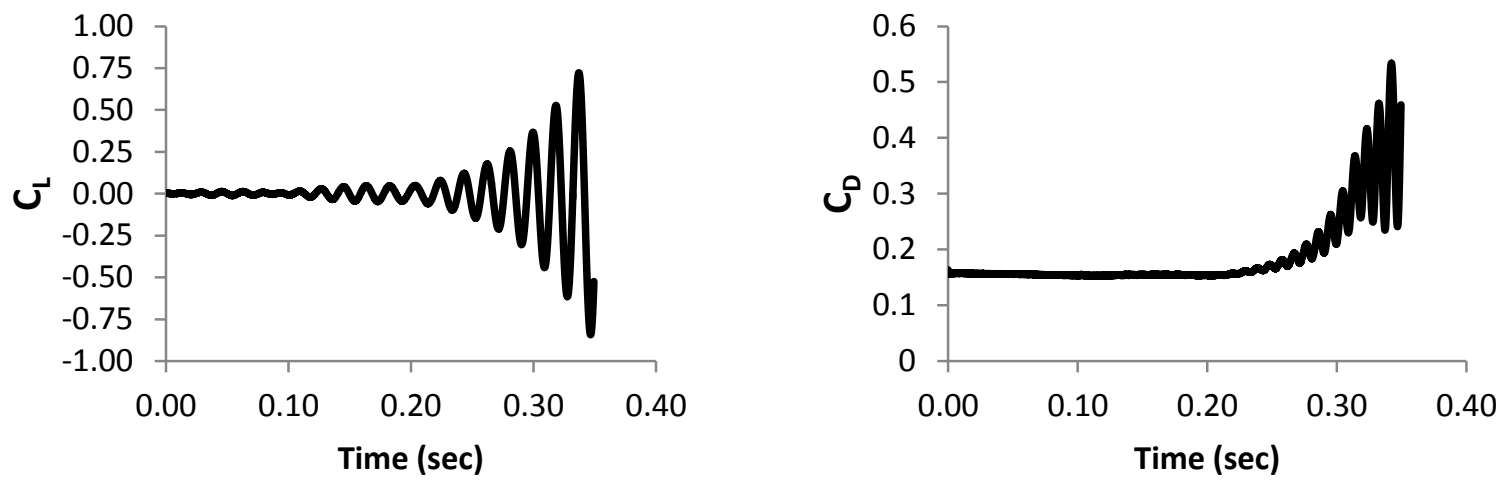

(a)

(b)

Figure 6-4: $40 \mathrm{~mm}$ Baseline Configuration Coefficient Magnitudes of (a) Aerodynamic Lift and (b) Drag 


\subsection{Tail-deployed Configuration}

\subsubsection{Sensitivity Analysis}

Similar to the baseline configuration, a sensitivity analysis was performed on the fin stabilized projectile configuration. Two numerical flight tests were conducted for approximately 1,000 time steps at 70 iterations per time step and again at 15 iterations per time step. As shown below in Figure 6-5, the drag differed by less than $2.5 \%$. Similar to the baseline configuration, the computational expense of this simulation was reduced significantly.

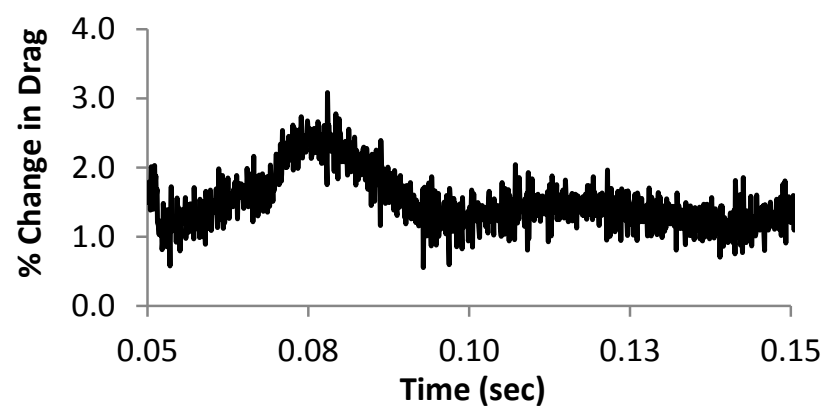

Figure 6-5: Sensitivity Analysis of Tail-deployed Configuration for Numerical Flight Testing

\subsubsection{Stability Characteristics}

The aerodynamic behavior of the fin-stabilized projectile undergoing free body motion was analyzed for a total simulation time of $2 \mathrm{sec}$. Because the total flight time was considerably greater in comparison to the baseline round, the simulation of the tail-deployed configuration was much more computationally expensive. That is, the simulation required approximately 35 days or 5,000 CPU hours of computational time.

A major requirement for the tail-deployed configuration was to examine the rate of spin decay and time required to de-spin. In order to evaluate de-spin, four different techniques were considered. The first technique used the coupled CFD/6-DOF simulation tool to predict the spin decay rate of the finned projectile while in free flight motion. As provided in Figure 6-6, the numerical flight test results predicted de-spin to occur in less than $1 \mathrm{sec}$. This rapid decrease can be contributed to the aerodynamic forces acting on the large surface area of the tail fins, which translates into a large spin damping moment. For comparison, a second approach was performed in which the coupled solver was used as well. However, all translational and rotational motions were constrained except for rotation about the projectile's roll axis. The finned projectile was given an initial roll rate of 3,600 RPM while imposing a constant free stream velocity of $70 \mathrm{~m} / \mathrm{s}$ in the flow field. Since the numerical flight test predicted a rapid decrease in spin rate without considerable change in the velocity magnitude, this method was expected to provide similar results. The other two techniques for evaluating de-spin used an analytical solution that was discussed earlier. The analytical solution assuming an elliptical lift distribution on the tail fins compared quite well with the two computational techniques. In addition to the spin rate, the velocity magnitude dropped by approximately $20 \mathrm{~m} / \mathrm{s}$, as shown in Figure 6-6.

Figure 6-7 provides the longitudinal and lateral motion in which the angle of attack and pitch attitude angle were plotted together as well as the sideslip and heading angles. The data shown for the pitch represents how much the projectile has pitched downward from the initial $45^{\circ}$ angle of launch. The angle of attack was plotted against the angle of sideslip, as shown in Figure 6-8. During projectile de-spin the angle of attack and angle of sideslip reached a maximum of approximately $2^{\circ}$. After de-spin, the angle of attack settled out at $-0.7^{\circ}$ and the angle of sideslip settled out at $0.5^{\circ}$. 

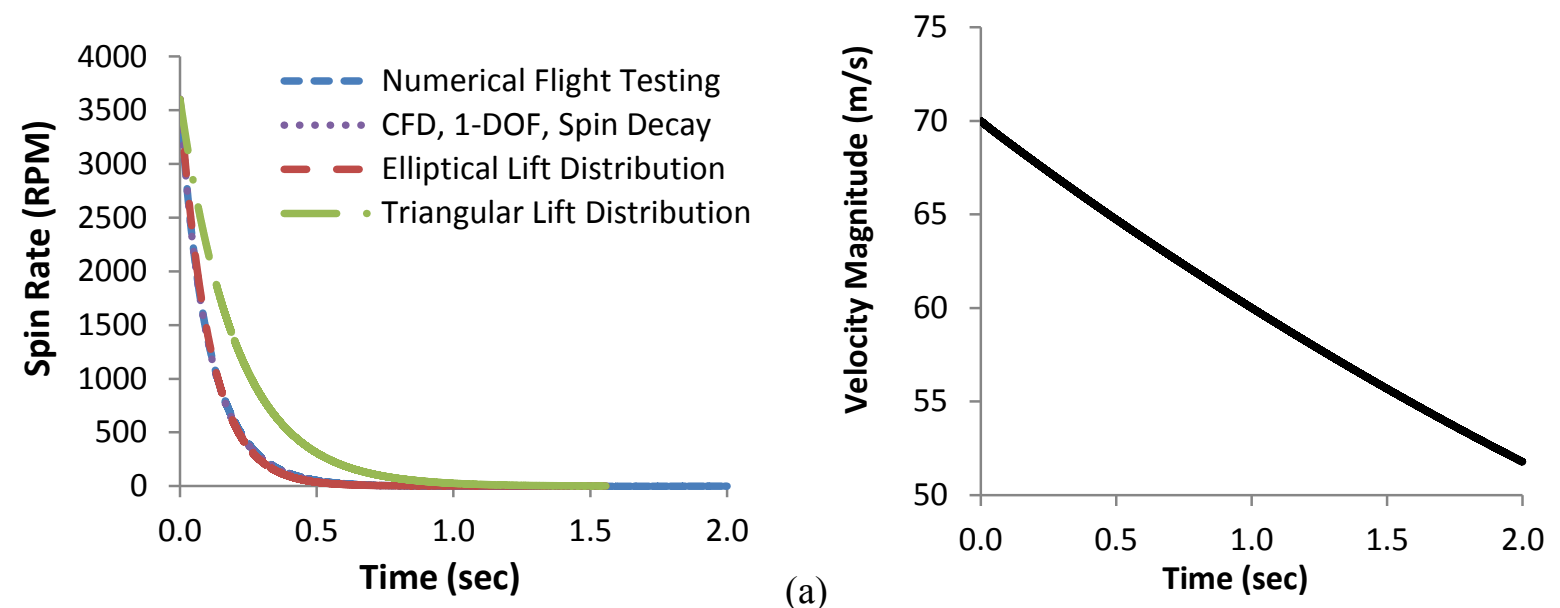

(a)

Figure 6-6: Tail-deployed Configuration Showing (a) Spin Rate Decay and (b) and Velocity Magnitude

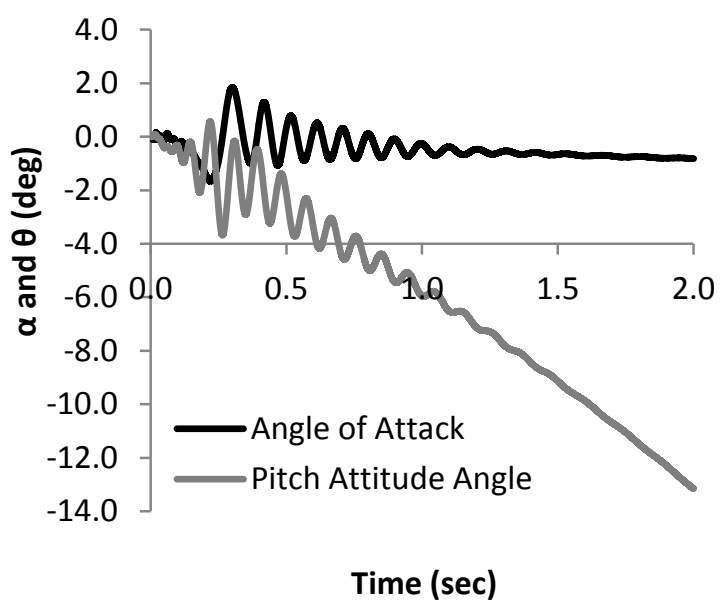

(a)

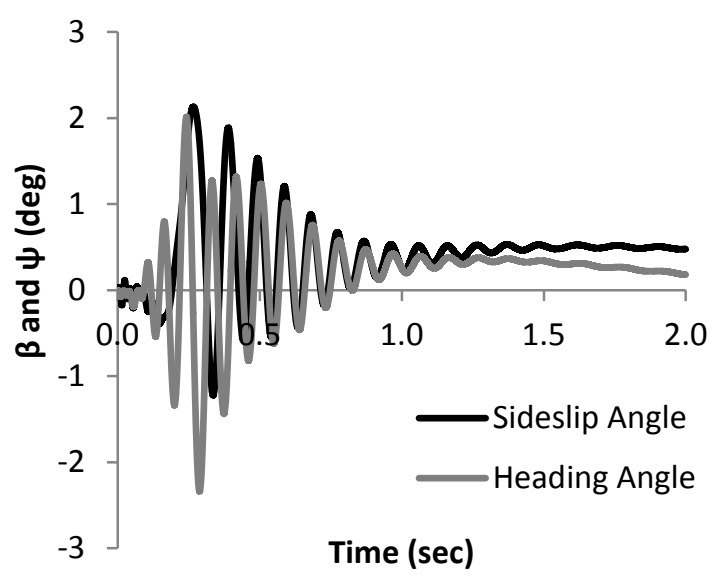

(b)

Figure 6-7: Tail-deployed Configuration Showing (a) Dampening of Longitudinal and (b) Lateral Motion

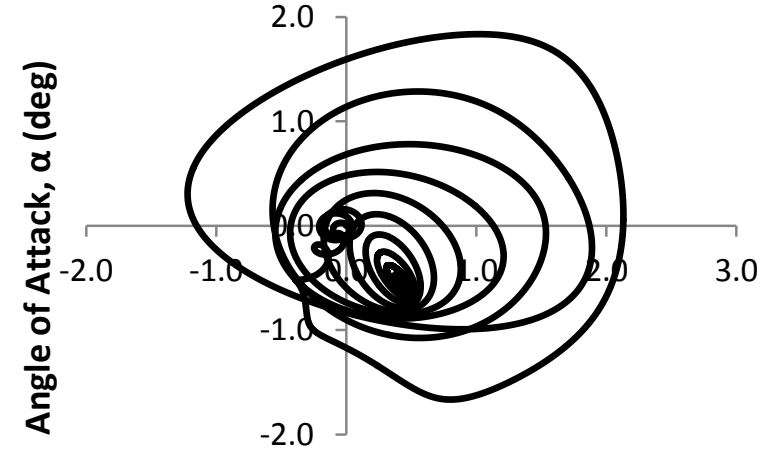

Angle of Sideslip, $\beta$ (deg)

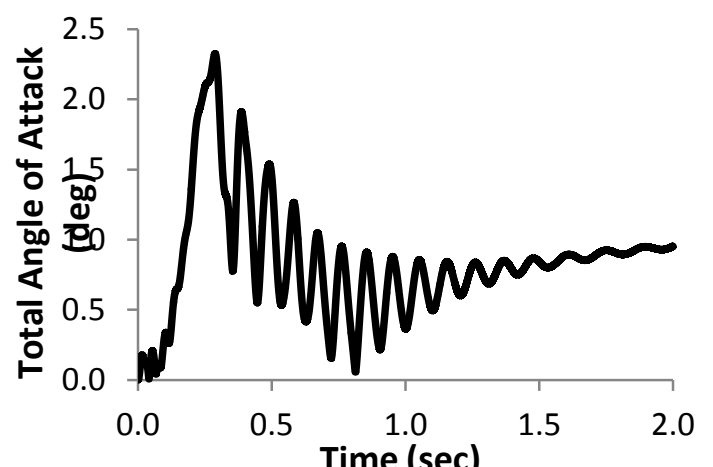

(a)

Figure 6-8: Tail-deployed Configuration Showing (a) $\alpha-\beta$ curve and (b) Total Angle of Attack

The contours of velocity magnitude were plotted at $0.01 \mathrm{sec}$ and again at $0.5 \mathrm{sec}$ after deployment of the fins. At $0.01 \mathrm{sec}$, the spin rate had dropped from 3600 RPM to 3300 RPM. At this spin rate, the 
wake extended far beyond the base of the projectile, as shown in Figure 6-9, due to vortex shedding. This can be more clearly shown in Figure 6-10 that illustrates the recirculating flow at the tips of the fins. Since the projectile was pushing the air out of its path, the maximum velocity occurred in the wake of the projectile at a magnitude of $90 \mathrm{~m} / \mathrm{s}$. In $0.5 \mathrm{sec}$ after fin deployment, the speed of the projectile had dropped to $65 \mathrm{~m} / \mathrm{s}$ and the spin decay had dropped to less than $50 R P M$. At such a low spin rate, the vortex shedding had decayed significantly, as illustrated in Figure 6-11. Also, the projectile's wake had reduced in size as a result of decreased amount of recirculating airflow. This can be shown in Figure 6-12 in which the velocity contours colored in green and light blue had contracted closer to the projectile base.

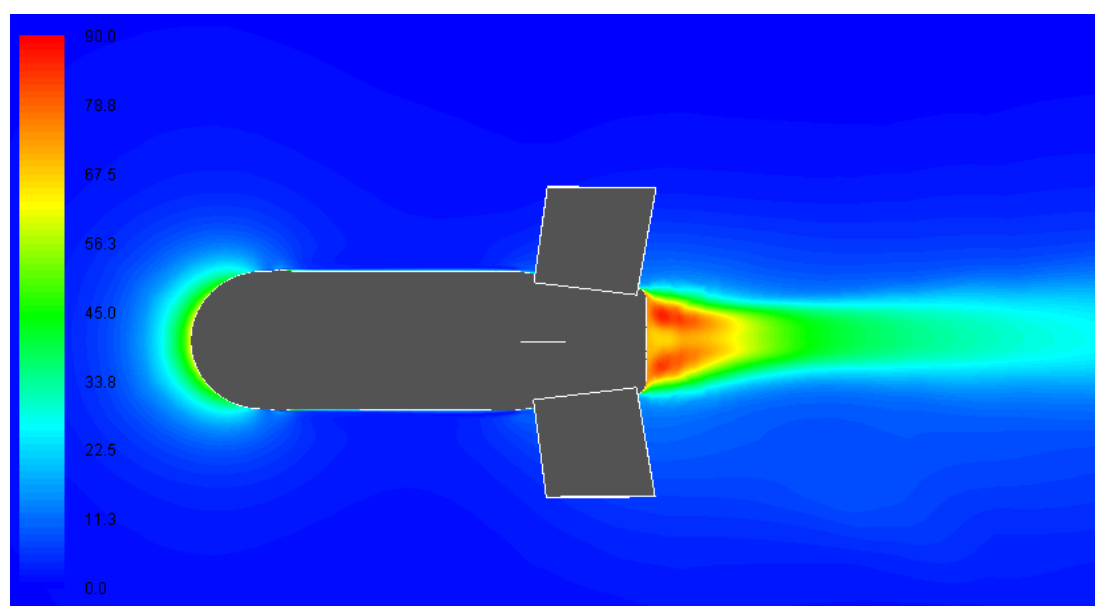

Figure 6-9: Velocity Contours of Tail-deployed Configuration Showing Wake Flow Immediately after being Launched from the Tube

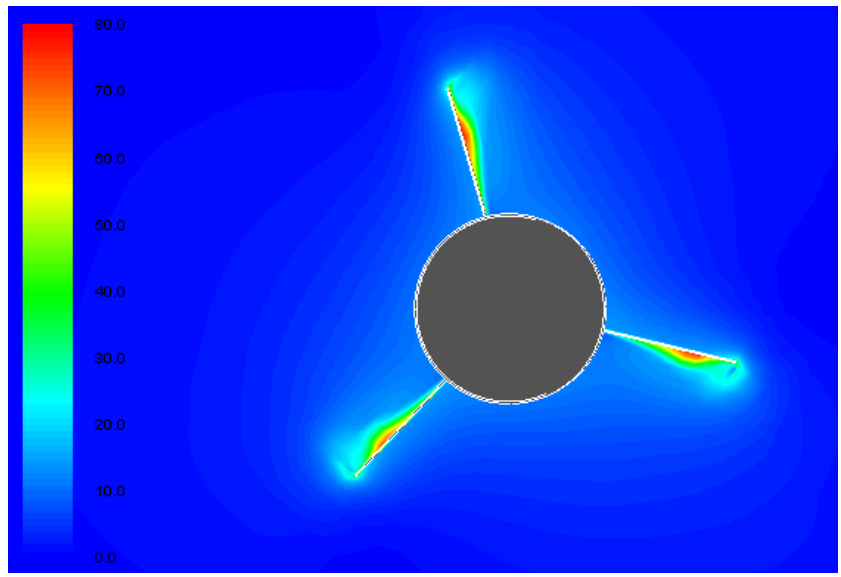

Figure 6-10: Velocity Contours of Tail-deployed Configuration Showing Vortex Shedding Immediately after Being Launched from the Tube 


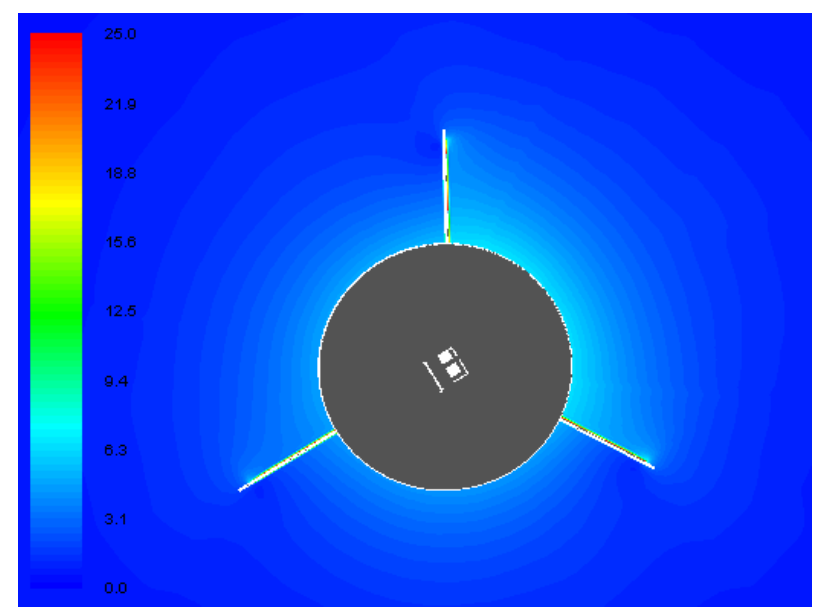

Figure 6-11: Velocity Contours of Fin-stabilized Projectile at 0.5 Second after Deployment of Tail Showing Decreased Recirculating Flow

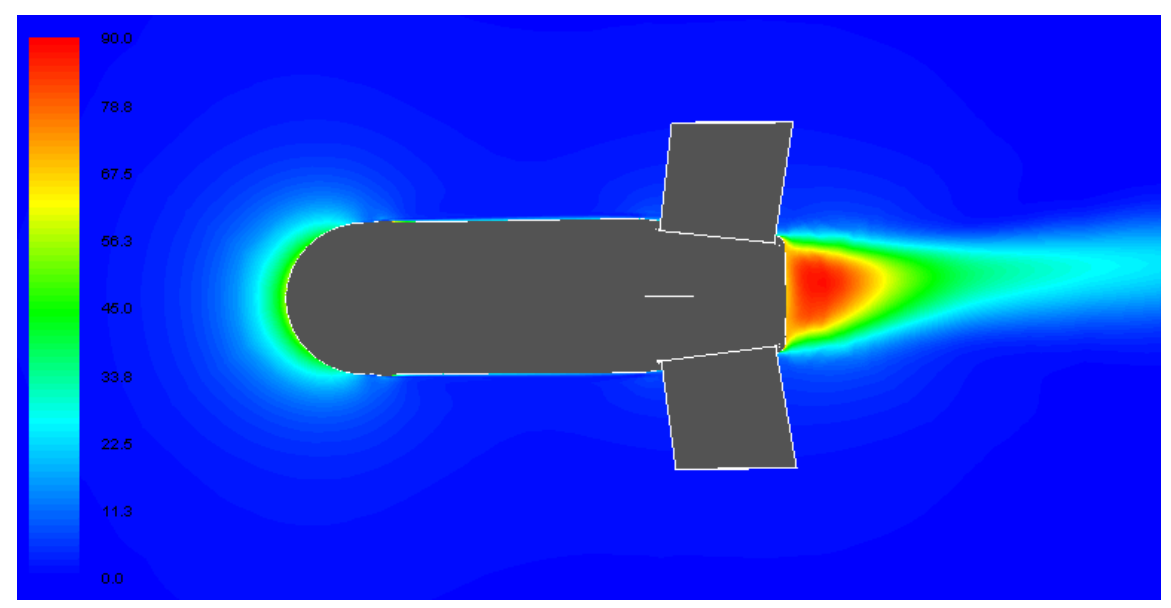

Figure 6-12: Figure 6-12: Velocity Contours of Fin-stabilized Projectile Showing Wake Flow at 0.5 Second after Deployment of Tail

\subsubsection{Aerodynamic Force Characteristics}

The characteristics of lift and drag were analyzed in addition to the longitudinal and lateral stability of the finned projectile. The two numerical simulations were performed to evaluate the drag during projectile de-spin in which the results showed good agreement, as provided in Figure 6-13. Initially, the drag was quite large with a coefficient magnitude of 0.3 . This was directly related to the large effective angle of attack on the tail fins, which was approximately $68^{\circ}$ for an initial spin rate of 3,600 RPM. As the spin rate decreased, the effective angle of attack decreased as well. Consequently, the drag dropped significantly and steadied out around a coefficient magnitude of 0.18 for the non free flight test simulation, as shown in Figure 6-13. This drag coefficient compared well with the traditional CFD simulation results of the finned projectile at $0^{\circ}$ AOA. Once the roll rate had dropped significantly, the numerical flight testing predicted a further drop in the drag. This reduction in drag was due to the projectile losing momentum as its altitude increased. The fluctuations were related to the variations in the angle of attack. Similarly, the lift initially fluctuated quite significantly due to the effective angle of attack on the tail fins. As the simulation continued, the amplitudes in the oscillations were reduced in which the lift generation was insignificant. 

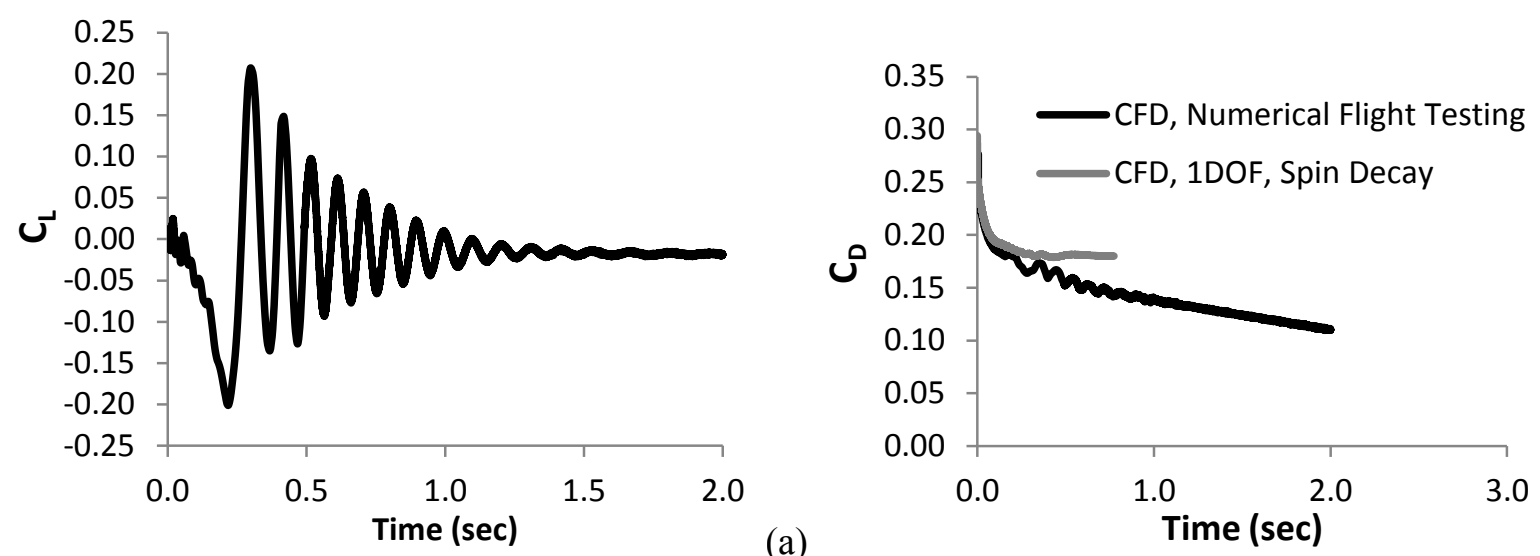

(a)

(b)

Figure 6-13: Aerodynamic Results for (a) Lift and (b) Drag on Tail-deployed Configuration

\subsection{Fully Deployed MAV Configuration}

\subsubsection{Sensitivity Analysis}

For the inverted Y-tail design of the fully deployed MAV configuration, a sensitivity analysis was performed to obtain the lift and drag. Two simulations were executed for approximately 350 iterations in which the first simulation was conducted for 70 iterations per time step and again for 15 iterations per time step. Figure 6-14 shows that the lift and drag differed by less than $1 \%$ and $2 \%$, respectively. Similar to the previous configurations, the numerical flight test was executed at 15 iterations per time step. A sensitivity analysis of the Y-tail design was not performed since the geometric shape and mesh size were nearly identical to the inverted Y-tail design.

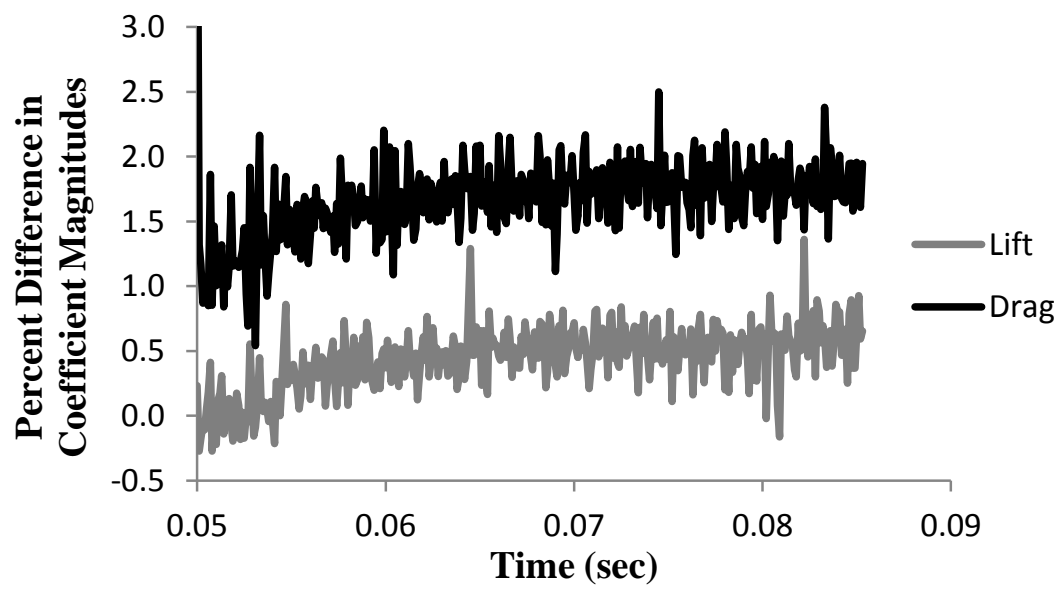

Figure 6-14: Sensitivity Analysis of Fully Deployed MAV Configuration for Numerical Flight Testing

\subsubsection{Inverted Y-tail Design}

The numerical flight test results for the aerodynamic behavior of the inverted Y-tail configuration were analyzed. The total simulation time was for approximately $3.15 \mathrm{sec}$ in which the MAV traveled 136 meters downrange. The numerical flight testing conducted on the wings-deployed configuration was significantly more computationally expensive than any of the previous simulations. That is, the simulation required approximately 55 days or $7875 \mathrm{CPU}$ hours of computational time. The initial velocity of the 
MAV at deployment of the wing was $45 \mathrm{~m} / \mathrm{s}$ and after $3 \mathrm{sec}$ of flight, the velocity of the MAV dropped to $37 \mathrm{~m} / \mathrm{s}$, as shown in Figure 6-15.

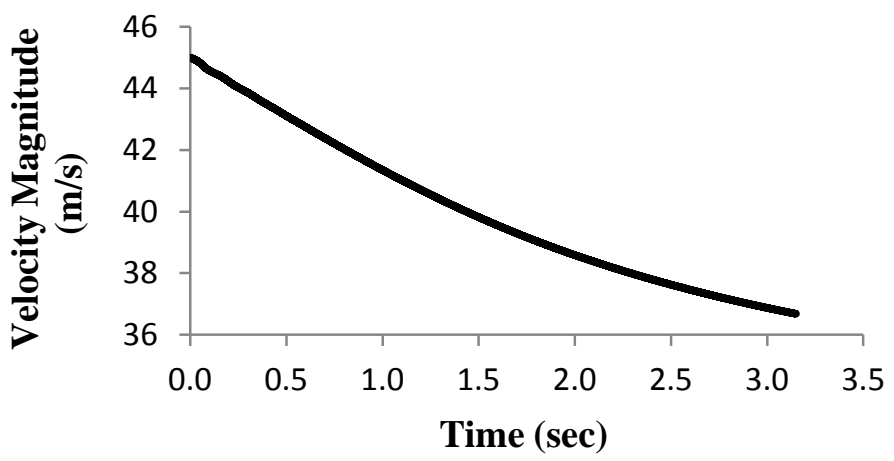

Figure 6-15: Velocity Magnitude for Inverted Y-tail Design of Fully Deployed MAV Configuration

\subsubsection{Longitudinal and Lateral-Directional Stability}

The longitudinal and lateral-directional stability of the inverted Y-tail configuration was examined. As shown in Figure 6-16, the numerical flight test predicted that the short period oscillations in the longitudinal dynamics lasted for less than 1 second. The angle of attack steadied out around $4.5^{\circ}$. Unlike the longitudinal stability, instability was present in the lateral-directional dynamics. This was a direct result of the destabilizing inverted Y-tail design. As provided in Figure 6-17, the MAV banked as much as $42^{\circ}$ viewed from the rear looking downrange at approximately $1.6 \mathrm{sec}$ after deployment of the wing and returned to its equilibrium position after $3 \mathrm{sec}$. The yawing was less significant in that the MAV yawed a maximum of $18^{\circ}$ at 2.7 after wing deployment, as shown in Figure 6-18. In analyzing the data, it was apparent that the rolling and yawing dynamics were coupled in which the presence of a large dihedral effect was being compensated by the $5^{\circ}$ wing dihedral. Figure 6-19 presents the numerical flight test results for the angle of sideslip. In comparison to the angle of attack, the angle of sideslip was considerably less since the sideways component of velocity was small in relation to the velocity magnitude.
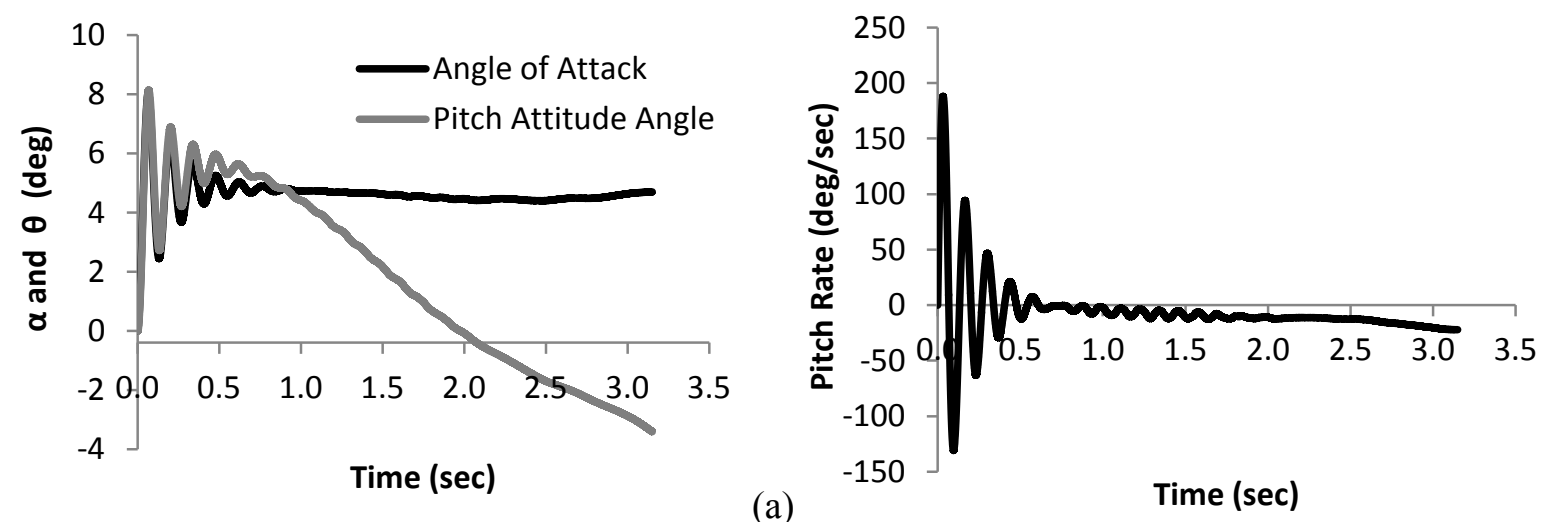

Figure 6-16: Longitudinal Dynamics of Inverted Y-tail Configuration (a) Angle of Attack and Pitch Attitude Angle and (b) Pitch Rate 

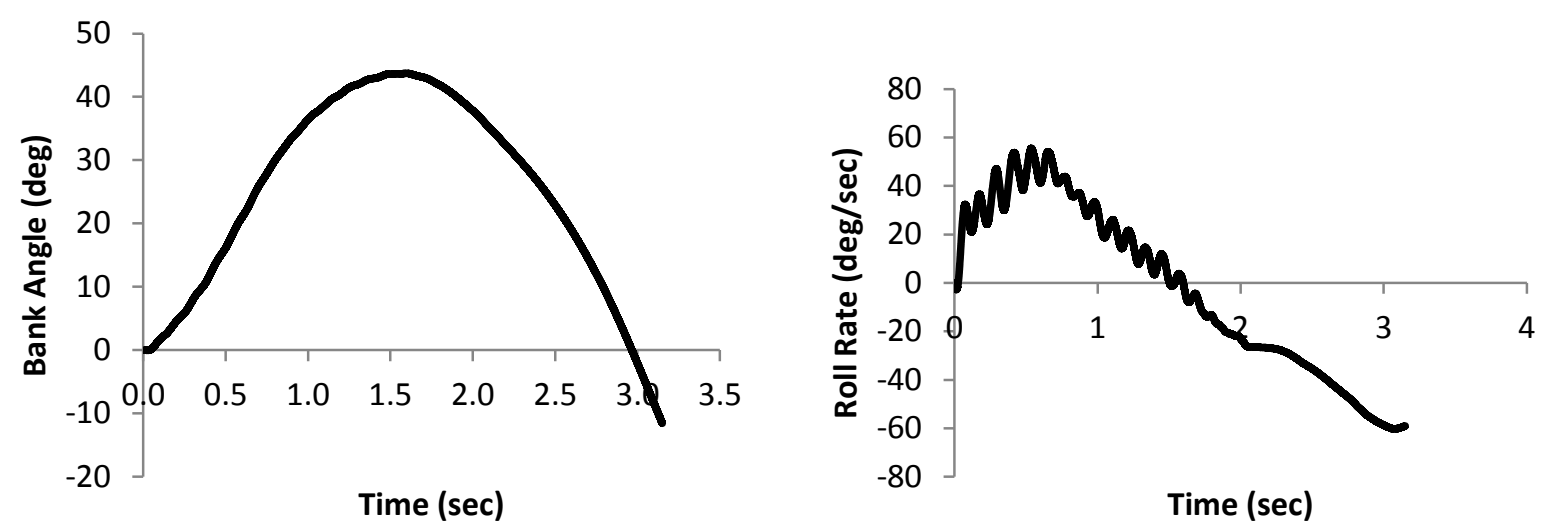

Figure 6-17: Lateral Dynamics of Inverted Y-tail Configuration (a) Bank Angle and (b) Roll Rate
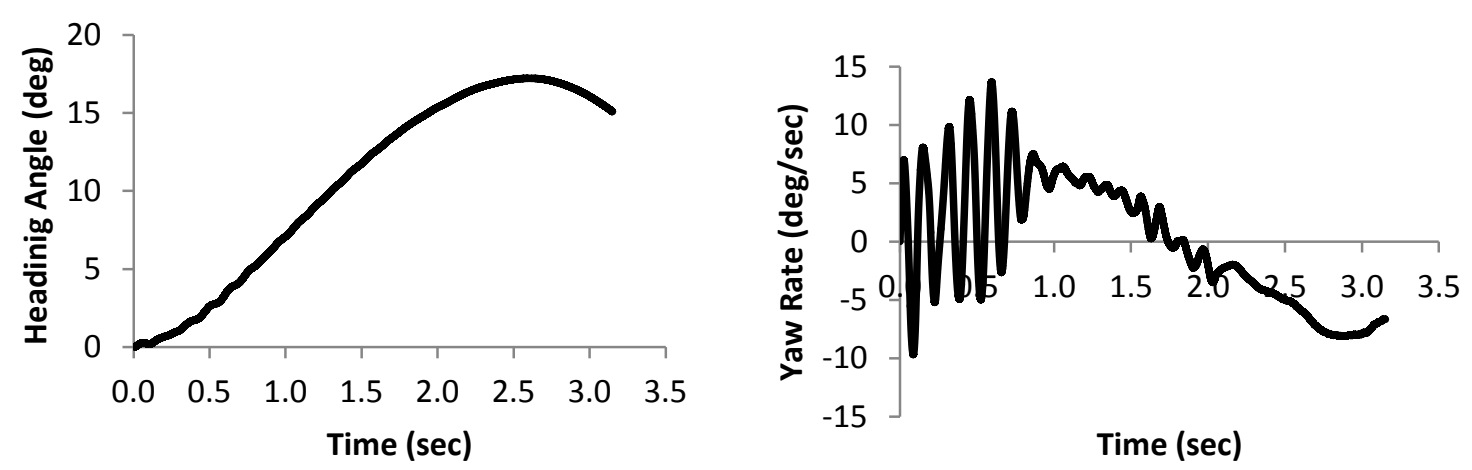

(b)

Figure 6-18: Lateral Dynamics of Inverted Y-tail Configuration (a) Heading Angle and (b) Yaw Rate

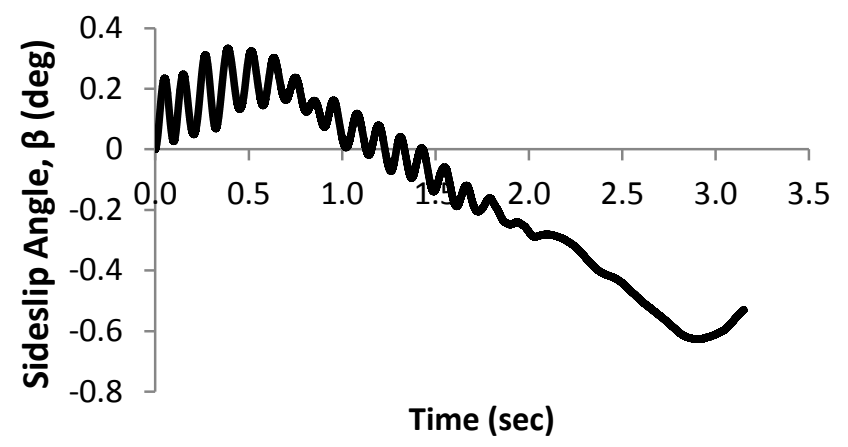

Figure 6-19: Angle of Sideslip for Inverted Y-tail Configuration

\subsubsection{Aerodynamic Force Characteristics}

The characteristics of lift and drag were analyzed in addition to the longitudinal and lateraldirectional stability. During the short period oscillations, the lift coefficient reached a maximum of 2.5. This was directly related to the angle of attack in which the maximum lift was generated at $8^{\circ} \mathrm{AOA}$. As the MAV entered into its long period or phugoid mode, a reduction in lift was noticeable at approximately $1.6 \mathrm{sec}$ after executing the simulation. This was due to the MAV banking $42^{\circ}$ to the right in which the vertical lift component of the total lift generated on the MAV was decreasing. As the MAV began 
banking to the left, the vertical lift component began increasing again. Similarly, the short period oscillatory motion in the drag occurred for the same time duration with a maximum drag of 0.66 , as shown in Figure 6-20. Once the short period oscillations were damped out, the drag decreased due to the reduction in the velocity. The numerical flight test results showed the magnitude of lift-to-drag generated on the inverted Y-tail configuration was approximately 3, also provided in Figure 6-20.

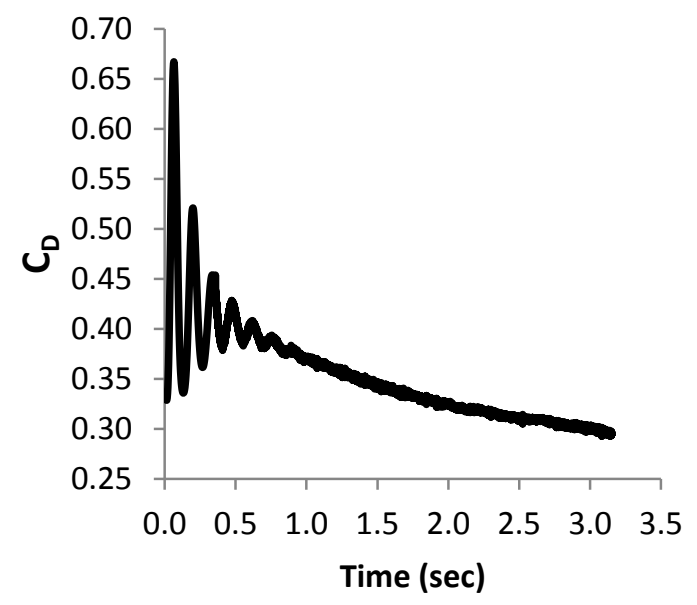

(a)

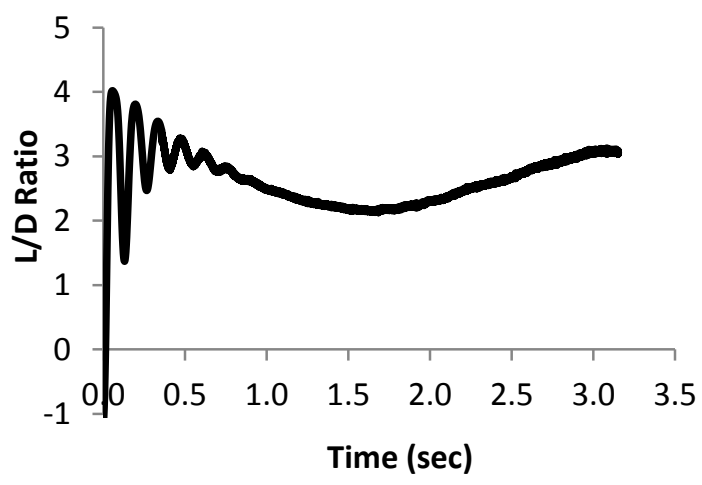

Figure 6-20: Inverted Y-tail Configuration Data for (a) Aerodynamic Drag and (b) Lift-to-Drag

At $0.08 \mathrm{sec}$ after deployment of the wing, the angle of attack was slightly greater than $7^{\circ}$. The contours of velocity magnitude were displayed in Figure 6-21 to show that the maximum velocity of 65 $\mathrm{m} / \mathrm{s}$ occurred behind the base of the MAV. At this same instance in time, the pathlines of velocity magnitude were displayed along the trailing edge of the wing to show the vorticity being generated at the wing tips. This was expected because the high pressure air underneath the wing would spill over into the lower pressure air above the wing, as provided in Figure 6-22. As shown in Figure 6-23, an additional plot of the velocity pathlines was presented to show the wing tip vorticity at the maximum bank angle of $42^{\circ}$.

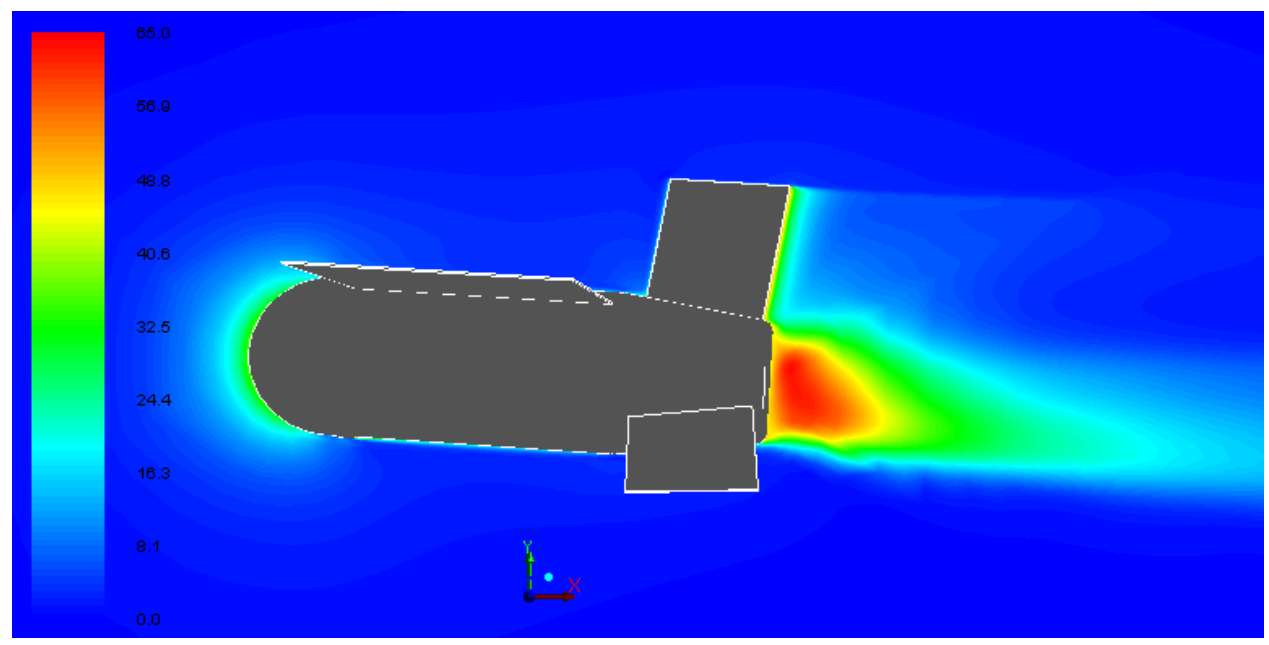

Figure 6-21: Velocity Contours of Inverted Y-tail Configuration Showing Wake Flow Immediately after Deployment of Wing 


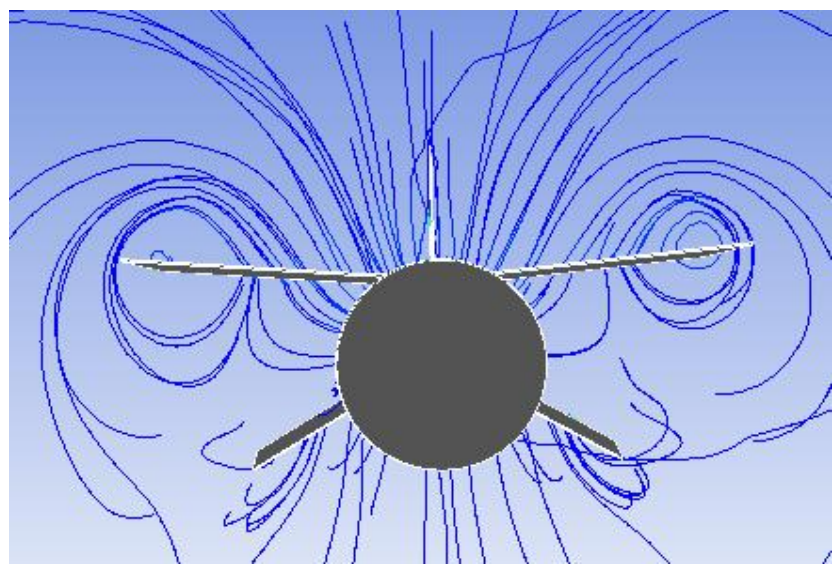

Figure 6-22: Velocity Contours Showing Wingtip Vorticity for Inverted Y-tail Configuration Immediately after Deployment of Wing

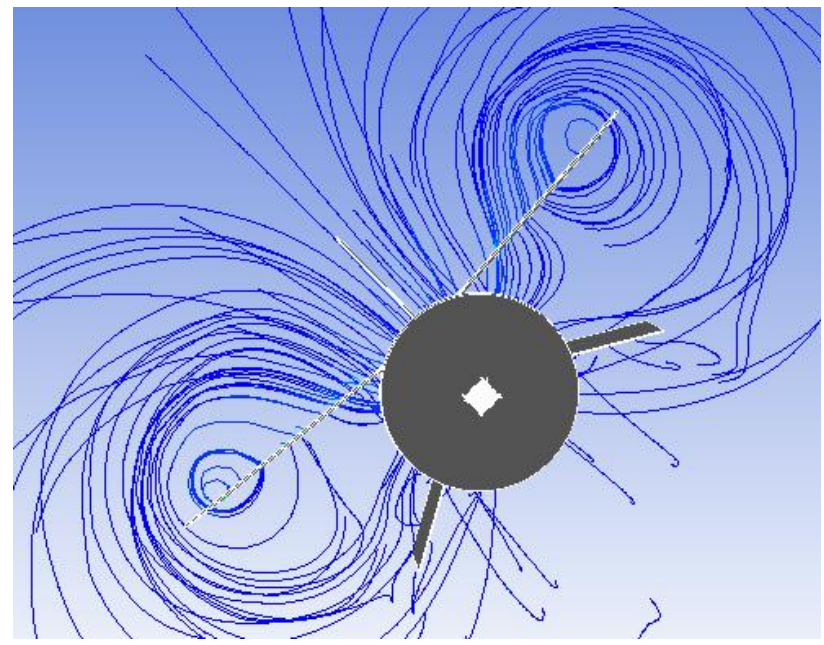

Figure 6-23: Velocity Contours Showing Wingtip Vorticity for Inverted Y-tail Configuration at $42^{\circ}$ Bank Angle

\subsubsection{Y-tail Design}

\subsubsection{Longitudinal and Lateral-Directional Stability}

The numerical flight test results for the aerodynamic behavior of the Y-tail configuration were analyzed for a tail deflection of $7.5^{\circ}$. The simulation was executed for approximately $0.91 \mathrm{sec}$ in which the MAV traveled 37 meters downrange. After analyzing the longitudinal stability, the numerical flight test predicted that it required considerably more time for the short period oscillations to damp out. This can be clearly shown in Figure 6-24, which provides the pitch attitude angle and angle of attack. The fluctuations shown in the data were related to the downwash from the wing causing a larger angle of attack on the body in comparison to the fixed body CFD analysis results. This generated instability in the pitch attitude angle and therefore, it was unnecessary to run the simulation any further. 

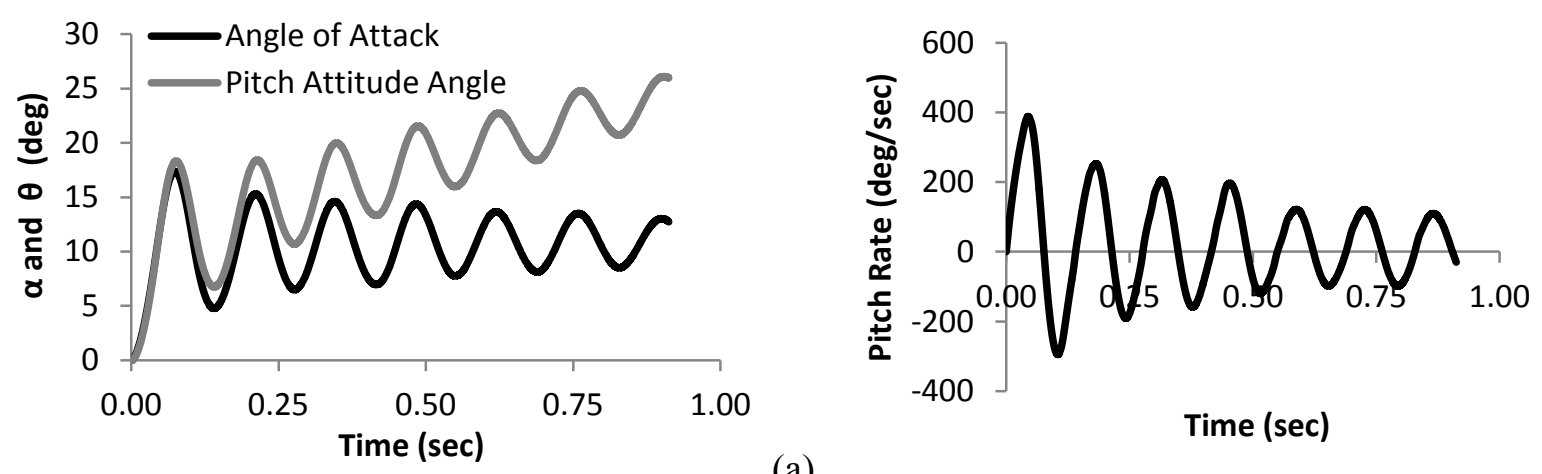

(a)

(b)

Figure 6-24: Longitudinal Dynamics of Y-tail Configuration for $7.5^{\circ}$ Tail Deflection Showing

(a) Pitch Attitude Angle and Angle of Attack and (b) Pitch Rate

Because of the instability issues in the longitudinal dynamics, another simulation was performed to evaluate the longitudinal and lateral-directional dynamics of the Y-tail configuration for no tail deflection. The angle of attack was oscillating for the same reason as described above; however, it was considerably less with no appearance of dampening, as shown in Figure 6-25. Additionally, this caused the pitch attitude angle to show no signs of dampening either. Conversely, the Y-tail configuration showed a significant improvement in its lateral-directional stability. The dihedral effect was considerably less in which the MAV banked a maximum of $3.5^{\circ}$ and yawed a maximum of $0.5^{\circ}$. This was directly related to the tail having a positive dihedral angle. The dynamics in the rolling and yawing motion are provided in Figure 6-26.
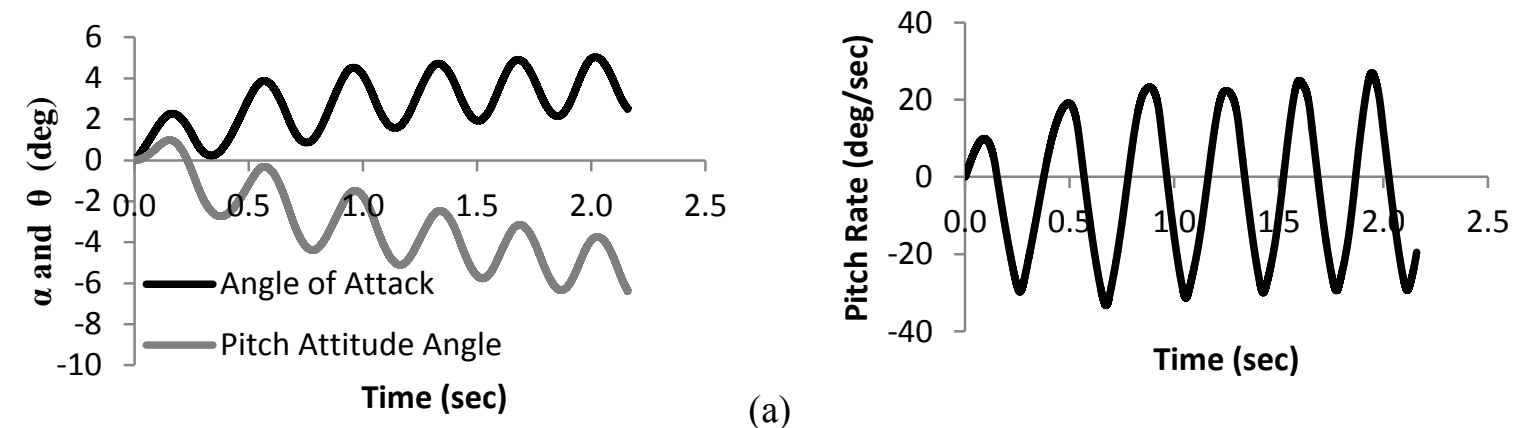

(a)

Figure 6-25: Longitudinal Dynamics of Y-tail Configuration for $0^{\circ}$ Tail Deflection Showing (a) Pitch Attitude Angle and Angle of Attack and (b) Pitch Rate 


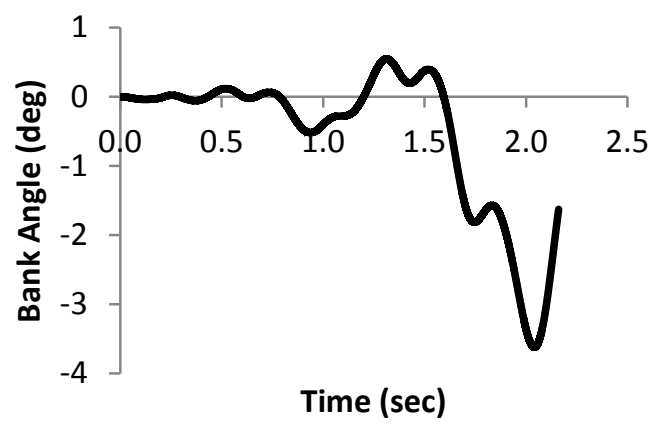

(a)

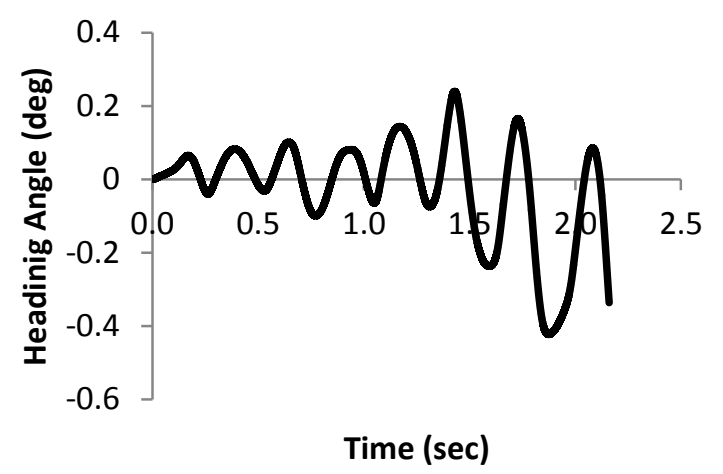

Time (sec)

(b)

Figure 6-26: Figure 6-29: Lateral Dynamics of Y-tail Configuration Showing Motion History of (a) Bank Angle and (b) Heading Angle

\subsubsection{Aerodynamic Force Characteristics}

The instability in the longitudinal dynamics affected the lift and drag on the Y-tail configuration. As shown in Figure 6-27, the magnitude of the drag coefficient oscillated between 0.25 and 0.38 . This was related to the variation in the angle of attack. Also, the lift coefficient data had the same period of oscillations but was greater in magnitude. The ratio of lift-to-drag appeared to have an upper limit of 3.5 and the lower limit increased upon each successive oscillation as a result of the slight increase in angle of attack. Figure 6-28 provides the velocity contours viewed from the side of the MAV that shows the still air being pushed out of the way. Similar to the inverted Y-tail configuration, the maximum velocity occurred within the wake of the MAV at a velocity magnitude of $55 \mathrm{~m} / \mathrm{s}$. The velocity pathlines were displayed at the trailing edge of the wing, as shown in Figure 6-29, to illustrate the vorticity being generated at the wingtips on the fully deployed MAV.
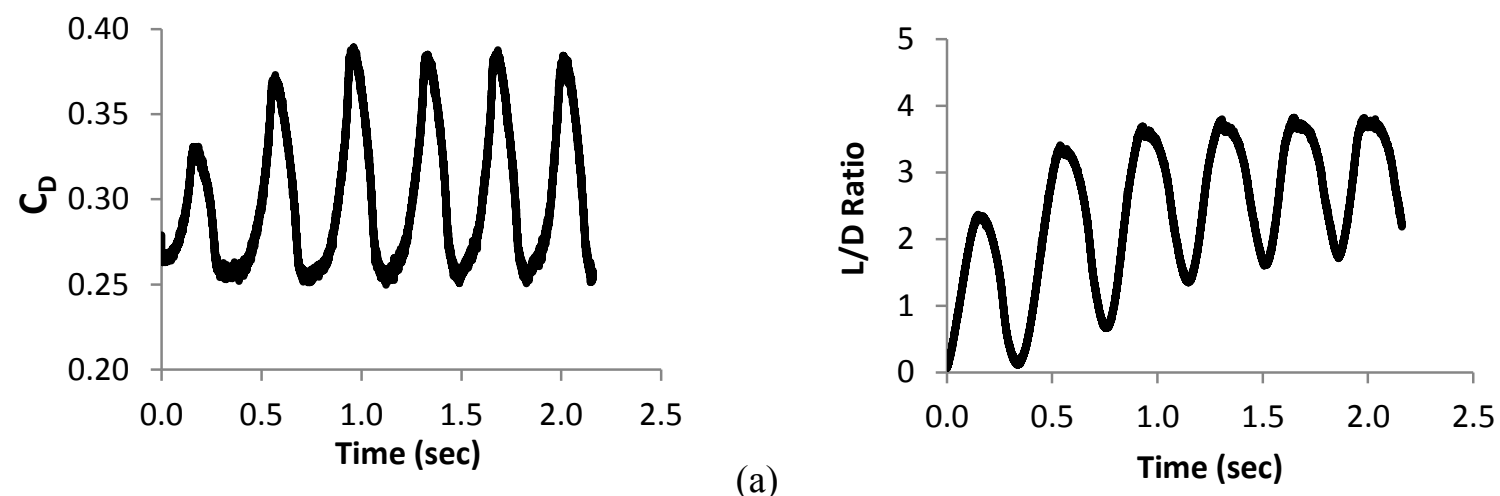

(a)

Figure 6-27: Y-tail Configuration Data for (a) Aerodynamic Drag and (b) Lift-to-Drag 


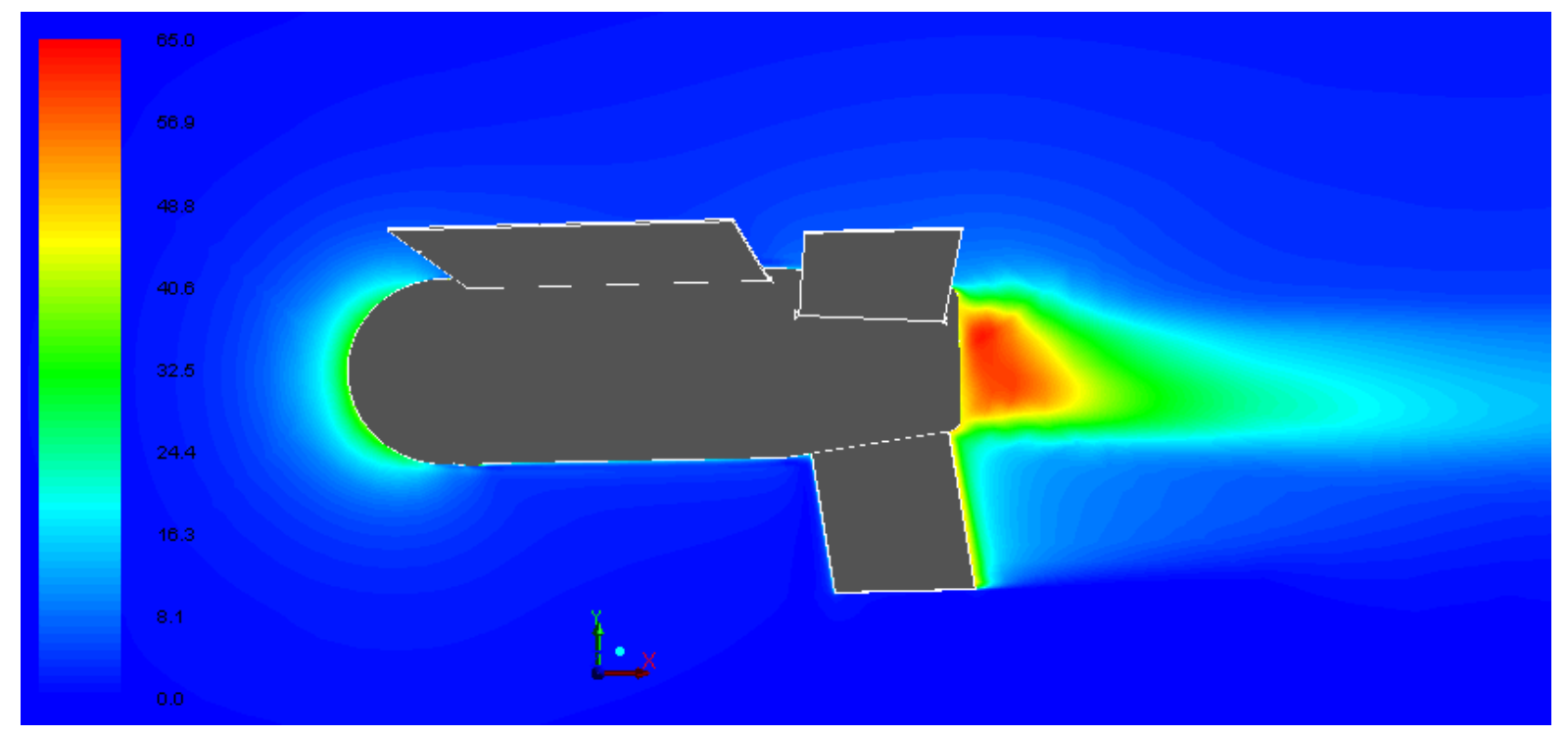

Figure 6-28: Velocity Contours of Y-tail Configuration Showing Wake Flow Immediately after Deployment of the Wing

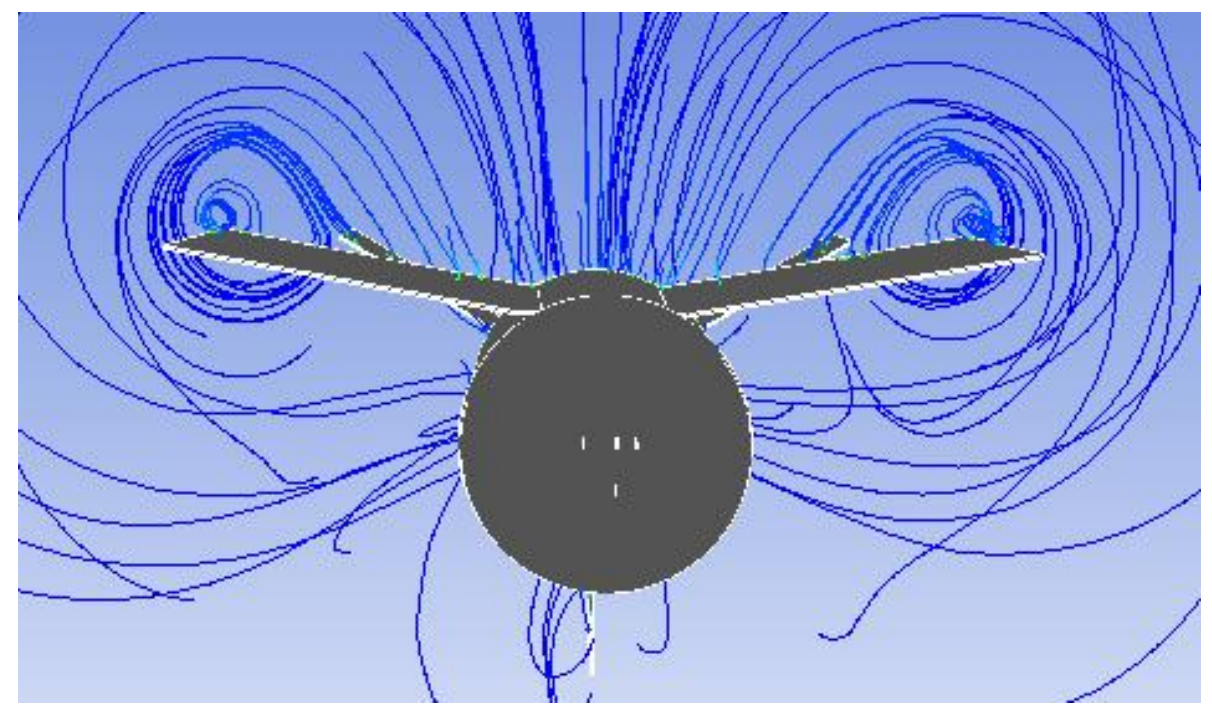

Figure 6-29: Velocity Pathlines of Y-tail Configuration Showing Wing Tip Vorticity Immediately after Deployment of the Wing 


\section{Chapter 7 Summary and Recommendations}

\subsection{Summary}

This study incorporated a relatively new simulation tool that coupled a CFD analysis to a 6-DOF module to predict the rigid body flight dynamics of a tube-launched transformable micro air vehicle. The use of this simulation tool is what this research is referring to as numerical flight testing. Initially, the MAV was launched from the tube in the baseline configuration followed by a first stage deployment of its tail fins for improved stability and shorter de-spin time. At the apex of the trajectory, the MAV had a second stage deployment of its wings for improvements in both range and accuracy. Two different tail configurations were considered for the fully deployed MAV that included a Y-tail design and an inverted Y-tail design. Overall, the coupled CFD/6-DOF simulation tool provided reasonable predictions of the MAV's free flight motion; however, the simulations required considerable amount of computational time that prevented the numerical flight testing from being carried out further.

The free flight motion analysis performed on the $40 \mathrm{~mm}$ baseline round had undesirable stability issues. As a result, several simulations were executed that consisted of simplifying the baseline geometry, increasing the mesh resolution, increasing the number of iterations per time step, utilizing the in-viscid solution, and selecting the time step based on the Courant number. However, in all simulation runs of the baseline round, the in-flight conical motion was unrealistically over-predicted according to prior research studies. In contrast, the simulations of the tail-deployed and wings-deployed configurations provided reasonable predictions. The pitching and yawing dynamics of the $40 \mathrm{~mm}$ finned projectile closely resembled the dynamic behavior of a $25 \mathrm{~mm}$ finned projectile presented in a prior study. Additionally, the simulation in the current study demonstrated that the fin-stabilized projectile required approximately $1 \mathrm{sec}$ to de-spin, which agreed quite well with the analytical solution. The numerical flight test demonstrated that a finned projectile was not only a necessary design requirement because of its improved dynamic stability, but also for its rapid reduction in the spin rate before deploying the wings at the apex of the original ballistic trajectory.

The two tail designs considered in this study each caused the fully deployed MAV's flight characteristics to behave differently. The inverted Y-tail design generated a large Dutch roll mode resulting in considerable rolling and yawing motion, whereas the Y-tail design generated a smaller Dutch roll mode and therefore, it showed improved lateral-directional stability. Conversely, the numerical flight testing of the inverted Y-tail design demonstrated improved longitudinal stability. After examining both tail configurations, the inverted Y-tail design demonstrated better overall flight performance characteristics. That is, the short period oscillations in the MAV's longitudinal dynamics damped out rather quickly and provided a more desirable lift-to-drag ratio.

\subsection{Recommendations}

As an extension to the research already performed in this study, additional aerodynamics work should be addressed that could be beneficial to the future of the HP project. For simplicity, a bulleted list is provided below with recommendations in support of advancing the current research.

- Further investigate the over-predictions in dynamic instability on the $40 \mathrm{~mm}$ baseline round while undergoing free flight motion. Because this dynamic instability could be related to the incorrect moments of inertia for the projectile, these properties should be obtained experimentally and another numerical flight test performed using the updated moments of inertia. 
- Design models of the tail-deployed and wings-deployed configurations for conducting wind tunnel testing to measure the aerodynamic lift, drag, and pitching moment and validate the CFD results with experimental data

- Perform wind tunnel testing to measure the spin decay for the tail-deployed configurations and compare the experimental data with the results obtained from the numerical and analytical techniques

- Evaluate the finned projectile's spin decay and time required to de-spin for deforming tail fins by performing a simulation of a fluid-structure interaction

- Perform additional numerical flight test simulations on the inverted Y-tail configuration by initializing the MAV at different bank angles and then re-evaluate its longitudinal and lateraldirectional stability

- Shift the wings of the Y-tail configuration downward so that the tail fins are not in the path of the wing's wake and perform a numerical flight test to re-evaluate the longitudinal stability of the MAV 


\section{References}

ANSYS. (2012). Retrieved from ANSYS Fluent User Services Center: http://www.fluentusers.com/fluent/training/intermediate/tutorials/index.htm

ANSYS. (2012). ANSYS, Introduction. Retrieved from www.ansys.com

Carlucci, D. E., \& Jacobson, S. S. (2008). Ballistics: Theory and Design of Guns and Ammunition. Boca Raton, FL: CRC Press.

Chemring Group PLC. (2011). Retrieved September 2012, from Chemring Ordnance: http://www.chemringordnance.com/

Courant-Friedrichs-Lewy Condition. (2012, October 26). Retrieved November 27, 2012, from Wikipedia: The Free Encyclopedia: http://en.wikipedia.org/wiki/Courant\%E2\%80\%93Friedrichs\%E2\%80\%93Lewy_condition

DeSpirito, J., \& Heavey, K. R. (2004). CFD Computation of Magnus Moment and Roll Damping Moment of a Spinning Projectile. AIAA Atmospheric Flight Mechanics Conference and Exhibit. Providence, RI: American Institute of Aeronautics and Astronautics.

Hamburg, S. D. (2010). Conceptual and Prelimiiary Design of a Stowable Ruggedized Micro Air Vehicle. Morgantown, WV: West Virginia University.

John D. Anderson, J. (2007). Fundamentals of Aerodynamics, Fourth Edtion. New York, NY: McGrawHill.

Kelecy, F. J. (2008). ANSYS Advantage. Retrieved November 2012, from Coupling Momentum and Continuity Increases CFD Robustness:

http://www.ansys.com/staticassets/ANSYS/staticassets/resourcelibrary/article/AA-V2-I2Coupling-Momentum-and-Continuity.pdf

Khalil, M., Abdalla, H., \& Kamal, O. (2009). Trajectory Prediction for a Typical Fin Stabilized Artillery Rocket. 13th International Conference on Aerospace Sciences and Aviation Technology. Cairo, Egypt.

Lun, S. W. (2011). Aerodynamic Validation of Emerging Projectile Configuations. Monterey, CA: Naval Postgraduate School.

Lyon, D. H. (1997). Development of a 40-mm Nonlethal Cartridge. Defense Technology Federal Laboratories Research Journal , 64-78.

McCoy, R. L. (1999). Modern Exterior Ballistics: The Launch and Flight Dynamics of Symmetric Projectiles. Atglen, PA: Schiffer Publishing.

Murman, S. M., Aftosmis, M. J., \& Berger, M. J. (2003, January). Simulations of 6-DOF Motion with a Cartesian Method. AIAA Paper 2003-1246.

Panagiotopoulos, E. E., \& Kyparissis, S. D. (1990). CFD Transonic Store Separation Trajectory Predictions with Comparison to Wind Tunnel Investigations. International Journal of Engineering , 3 (6), 538-553.

RM Equipment Inc. (2011). Retrieved August 2012, from http://www.rm-equipment.com/

Roskam, J. (2003). Airplane Flight Dynamics and Automatic Flight Controls Part I. Lawrence, KS: DAR Corporation.

Sahu, J. (2006). Time-Accurate Computations of Free-Flight Aerodynamics of a Spinning Projectile With and Without Flow Control. Aberdeen Proving Ground, MD: Army Research Laboratory.

Sahu, J. (2005). Time-Accurate Numerical Prediction of Free Flight Aerodynamics of a Finned Projectile. Aberdeen Proving Ground, MD: Army Research Laboratory. 
SST K-omega Model. (2011, February 28). Retrieved July 2012, from CFD Online: http://www.cfdonline.com/Wiki/SST_k-omega_model

The k-omega and SST Models. (2010, August). Retrieved August 2012, from The Shared Hierarchical Academic Research Computing Network: https://www.sharcnet.ca/Software/Fluent13/help/cfx_mod/i1345900.html

Toledo, W. (2009). Determination of Spin Decay for a Finned Projectile. Unpublished Notes.

Valyou, D. N., Marzocca, P., Manole, L., \& Toledo, W. (2011). Experimental Methods for the Characterization of the Static and Dynamic Stability of a Spinning Body. Aerospace Technology Conference and Exposition. Toulouse, France: Society of Automotive Engineers. 


\section{Appendix A User Defined Functions for Free Body Motion Analysis}

The following source code was used for defining the MAV's translational motion along the negative $\mathrm{X}$-axis in order to establish a boundary layer around the body before initiating the 6-DOF solver. For the baseline and fin stabilized projectile, an angular velocity was defined about the negative X-axis was in addition to the translational motion. In looking downrange, the projectiles would have clockwise spin.

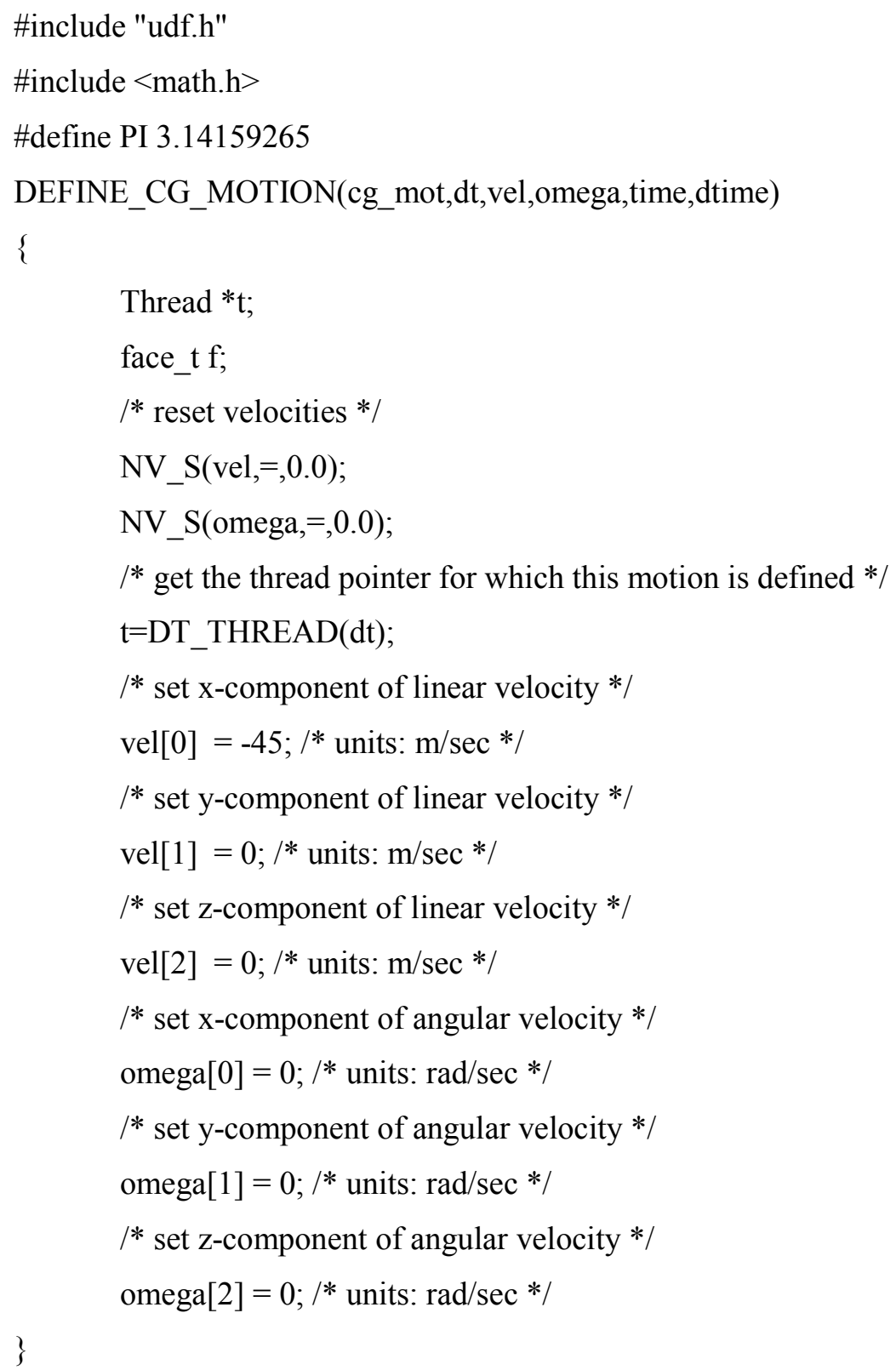


The following source code was the UDF for defining the mass and moments of inertia properties for the inverted Y-tail design of the fully deployed MAV configuration. Also, the UDF stored 13 parameters to a file that included the simulation time, CG position, CG linear velocity components, CG angular velocity components, and Euler (roll, pitch, yaw) angles.

\#include "udf.h"

DEFINE_SDOF_PROPERTIES(sdof_properties_wingtailfins, prop, dt, time, dtime)

\{

\# if !RP_NODE

FILE $* \mathrm{fp}=\mathrm{NULL}$;

char filename[]="udf_motion_history.txt";

if $((\mathrm{fp}=$ fopen(filename, $" \mathrm{a}+"))==\mathrm{NULL})$

Message("\n Warning: Unable to open \%s for writing \n", filename);

else

Message("\n Writing SDOF motion history to \%s...",filename);

\begin{tabular}{ll}
\multicolumn{1}{l}{$\begin{array}{l}\text { \# endif } \\
\text { prop[SDOF_MASS] }\end{array}$} & $=0.180 ; / *$ units: $\mathrm{kg} * /$ \\
prop[SDOF_IXX] & $=243811.70 * \operatorname{pow}(10,-9) ; / *$ units: $\mathrm{kg}^{*} \mathrm{~m}^{\wedge} 2 * /$ \\
prop[SDOF_IYY] & $=1192980.93 *$ pow $(10,-9) ; / *$ units: $\mathrm{kg}^{*} \mathrm{~m}^{\wedge} 2 * /$ \\
prop[SDOF_IZZ] & $=1183320.71 *$ pow $(10,-9) ; / *$ units: $\mathrm{kg}^{*} \mathrm{~m}^{\wedge} 2 * /$ \\
prop[SDOF_IXY] & $=11067.79 * \operatorname{pow}(10,-9) ; / *$ units: $\mathrm{kg}^{*} \mathrm{~m}^{\wedge} 2 * /$ \\
prop[SDOF_IXZ] & $=98.68 * \operatorname{pow}(10,-9) ; / *$ units: $\mathrm{kg}^{*} \mathrm{~m}^{\wedge} 2 * /$ \\
prop[SDOF_IYZ] & $=-13.63 * \operatorname{pow}(10,-9) ; / *$ units: $\mathrm{kg}^{*} \mathrm{~m}^{\wedge} 2 * /$
\end{tabular}

$/ *$ Determine whether the forces and moments are expressed in terms of body coordinates (TRUE) or global coordinates (FALSE) */

prop[SDOF_LOAD_LOCAL] $=$ FALSE;

\# if !RP_NODE

fprintf(fp, $\% \mathrm{~g} \quad \% \mathrm{~g} \quad \% \mathrm{~g} \quad \% \mathrm{~g} \quad \% \mathrm{~g} \quad \% \mathrm{~g} \quad \% \mathrm{~g} \quad \% \mathrm{~g} \quad \% \mathrm{~g} \quad \% \mathrm{~g} \quad \% \mathrm{~g} \quad \% \mathrm{~g} \quad \% \mathrm{~g} \backslash \mathrm{n}$ ",

CURRENT_TIME, DT_CG(dt)[0], DT_CG(dt)[1], DT_CG(dt)[2], DT_VEL_CG(dt)[0],

DT_VEL_CG(dt)[1], DT_VEL_CG(dt)[2], DT_OMEGA_CG(dt)[0], DT_OMEGA_CG(dt)[1],

DT_OMEGA_CG(dt)[2], DT_THETA(dt)[0]*180/M_PI, DT_THETA(dt)[1]*180/M_PI,

DT_THETA $(\overline{\mathrm{d}} \mathrm{t})[2] * 180 / \mathrm{M}$ - $\overline{\mathrm{I}})$;

fclose(fp);

\# endif

printf ("\n updated 6DOF properties"); 
The following source code was used for evaluating the spin decay of the fin-stabilized projectile in which the aerodynamic body was constrained to only allow rotation about its longitudinal axis while maintaining a constant free stream velocity. This analysis was performed to compare the rate of spin decay to that of the free body motion analysis.

\#include "udf.h"

DEFINE_SDOF_PROPERTIES(sdof_properties_tailfins, prop, dt, time, dtime)

\{

\# if !RP_NODE

FILE $* \mathrm{fp}=\mathrm{NULL}$;

char filename[]="udf_motion_history.txt";

if $((\mathrm{fp}=$ fopen(filename, $" \mathrm{a}+"))==\mathrm{NULL})$

Message("\n Warning: Unable to open \%s for writing \n", filename);

else

\{

Message("\n Writing SDOF motion history to \%s...",filename);

\}

\# endif

prop[SDOF_MASS] $=0.180 ; / *$ units: $\mathrm{kg} * /$

prop[SDOF_IXX] =34831.78*pow $(10,-9) ; / *$ units: $\mathrm{kg}^{*} \mathrm{~m}^{\wedge} 2 * /$

prop[SDOF_IYY] =354145.83*pow $(10,-9)$; /* units: $\mathrm{kg}^{*} \mathrm{~m}^{\wedge} 2 * /$

prop[SDOF_IZZ] =354145.83*pow $(10,-9)$; /* units: $\mathrm{kg}^{*} \mathrm{~m}^{\wedge} 2 * /$

$/ *$ Determine whether the forces and moments are expressed in terms of body coordinates (TRUE) or global coordinates (FALSE) */

prop[SDOF_LOAD_LOCAL] $=$ FALSE;

/* All projectile translation and rotation constrained except for rotation about X-axis) */

prop[SDOF_ZERO_TRANS_X] =TRUE;

$\operatorname{prop}[\mathrm{SDOF}$ ZZERO_TRANS_Y] =TRUE;

$\operatorname{prop}\left[\mathrm{SDOF} \_Z E R O \_T R A N S \_Z\right] \quad=$ TRUE;

prop[SDOF_ZERO_ROT_X] =FALSE;

$\operatorname{prop}\left[\mathrm{SDOF} \_\right.$ZERO_ROT_Y] $=$TRUE;

$\operatorname{prop}[\mathrm{SDOF}$ ZZERO_ROT_Z] =TRUE;

printf ("\n3d_particle: Updated 6DOF properties");

\# if !RP_NODE

fprintf(fp, $\% g \quad \% g \quad \% g \quad \% g \quad \% g \quad \% g \quad \% g \quad \% g \quad \% g \quad \% g \quad \% g \quad \% g \quad \% g \backslash n "$, CURRENT_TIME, DT_CG(dt)[0], DT_CG(dt)[1], DT_CG(dt)[2], 
DT_VEL_CG(dt)[0], DT_VEL_CG(dt)[1], DT_VEL_CG(dt)[2], DT_OMEGA_CG(dt)[0], DT_OMEGA_CG(dt)[1],DT_OMEGA_CG(dt)[2], DT_THETA(dt)[0]*180/M_PI, DT_THETA( $\overline{\mathrm{dt}})[1]^{*} 180 / \mathrm{M}$ PI,$\overline{D T}$ _THETA(dt)[2]*180/M_PI);

fclose(fp);

\# endif 
The following source code was used for calculating the three aerodynamic forces and three aerodynamic moments at every time step.

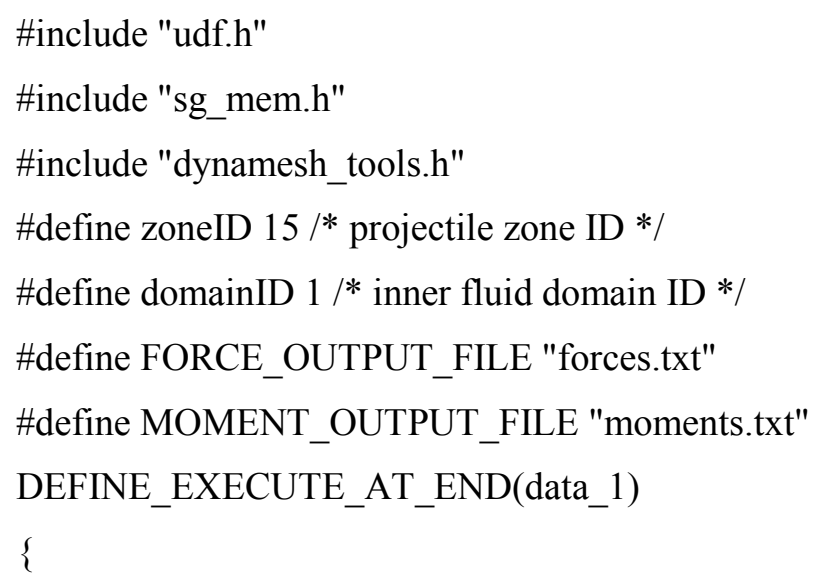

Compute_Force_And_Moment(domain,projectile,moment_center,f_glob,m_glob,TRUE);

fprintf(fd1,"\%g,\%g,\%g,\%g\n", RP_Get_Real("flow-time"), f_glob[0], f_glob[1], f_glob[2]); fclose(fd1); fprintf(fd2,"\%g,\%g,\%g,\%gln", RP_Get_Real("flow-time"), m_glob[0], m_glob[1], m_glob[2]); fclose(fd2); 


\section{Appendix B Configuration Details for 6-DOF Solver}

The configurations for the free body motion or 6-DOF simulations are presented using ANSYS Fluent R13. The figures shown below provide the configuration settings for the inverted Y-tail design of the fully deployed MAV configuration. A similar procedure was followed for the baseline and taildeployed configurations.

After loading the mesh into Fluent, it was first necessary to define the non-conformal interface between the structured and unstructured meshes. The user-defined functions (UDF) were then compiled and loaded in order to define the motion of the MAV. To build the UDFs, the Compiled UDFs window was opened by following the drop-down menu, as shown below in Figure B-1. For this simulation, there were three source files written in the C programming language. Figure B-2 shows three UDFs added to the source files field to be compiled and loaded. It was important for the source files to be located in the current directory where the case and data files were to be saved. The source code for the three UDFs can be found in Appendix A.

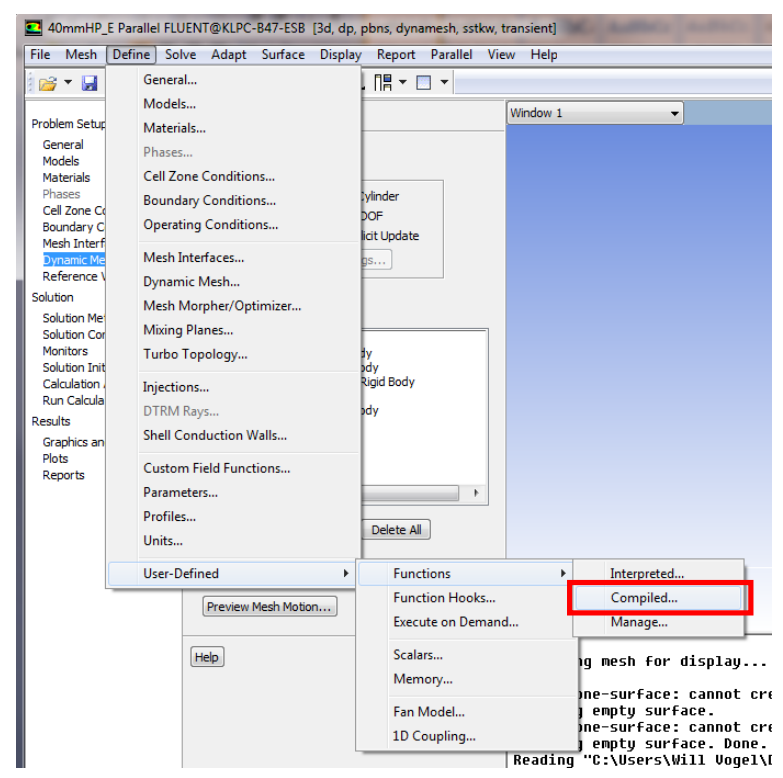

Figure B-1: Drop-down Menu for Compiling UDFs

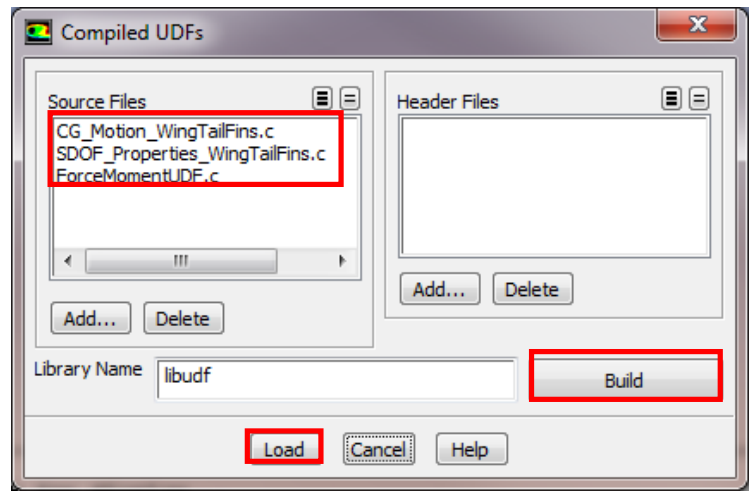

Figure B-2: UDFs Added to Source File List to be Compiled and Loaded 
The graphical user interface (GUI) for ANSYS Fluent R13 only allows two forces and 1 moment to be plotted and written to separate data files. In order to obtain all aerodynamic forces and moments, the third UDF listed in Figure B-2 was used to write the force and moment data to two separate files. Once the UDF was compiled and loaded, the function hooks were selected in the drop-down menu, as shown in Figure B-1. In Figure B-3, the UDF was hooked to the Execute at End function, which directed Fluent to record the aerodynamic forces and moments at the end of each time step.

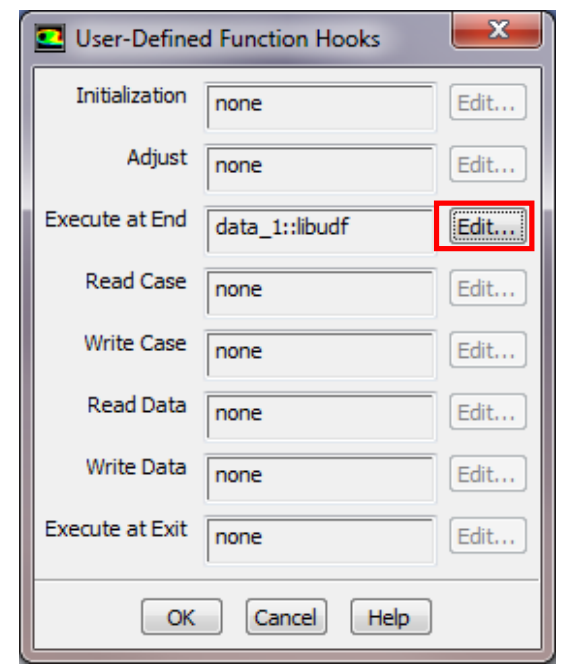

Figure B-3: UDF Hooked to Execute at End Function to Compute Forces and Moments

Once these settings were applied, the dynamic mesh feature was activated and the smoothing and remeshing mesh methods were selected, as shown in Figure B-4. As highlighted in Figure B-5, the settings for the smoothing and remeshing parameters are provided. The parameters for the smoothing settings were selected based on recommendations provided by ANSYS technical support and the parameters for the remeshing settings were based on the values provided in the mesh scale info.

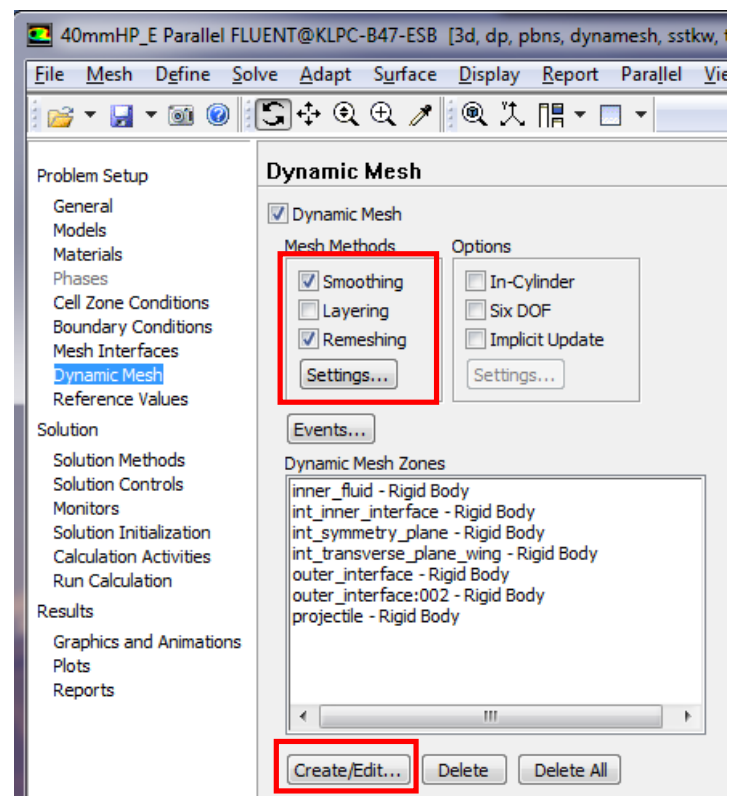

Figure B-4: Dynamic Mesh Menu Settings 

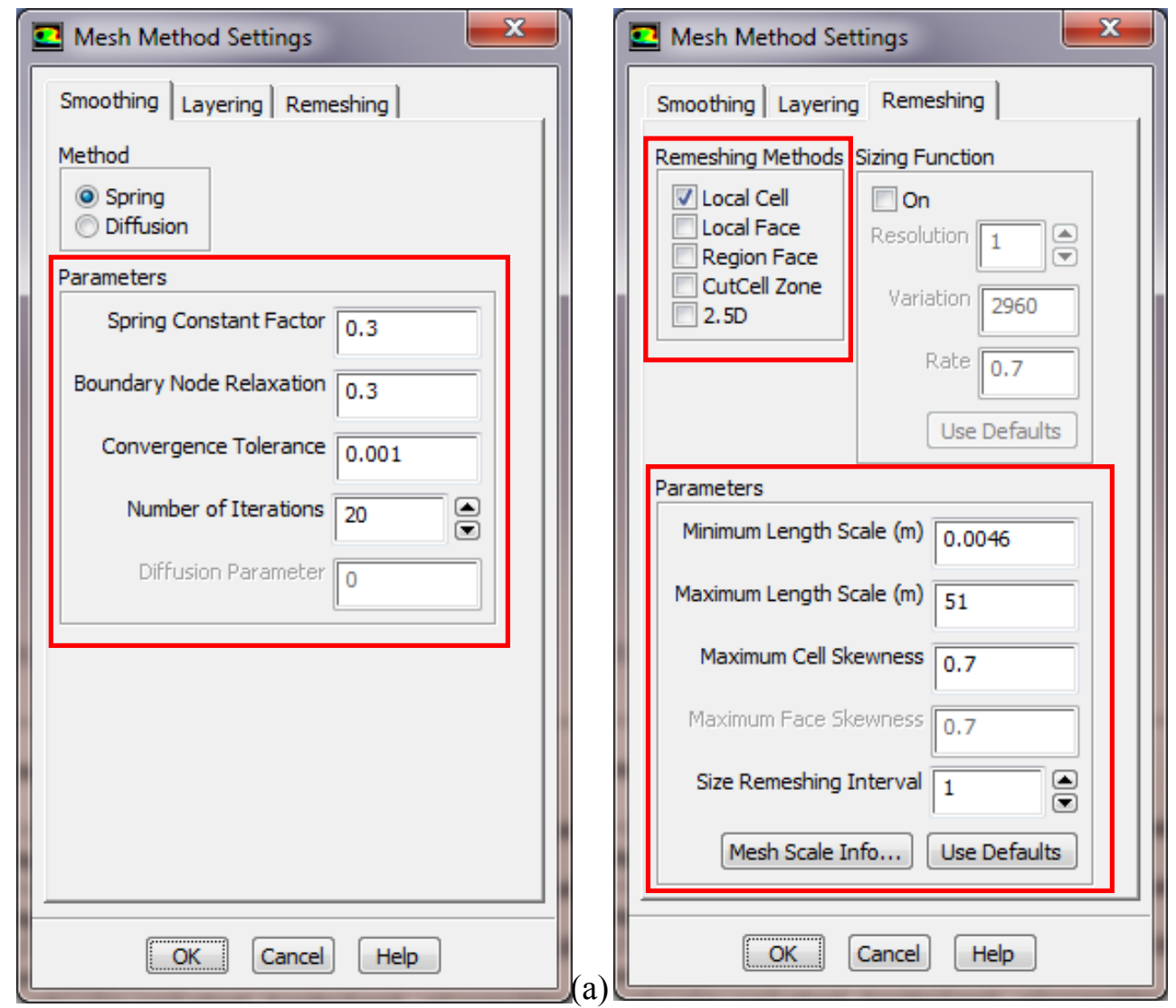

(b)

Figure B-5: Configuration Settings for (a) Smoothing and (b) Remeshing Parameters

The simulation was initiated by constraining the MAV to only translate along the negative X-axis of the fluid domain. The purpose of performing this simulation was to establish a boundary layer around the MAV before executing the 6-DOF solver. This required a UDF to define the velocity of the MAV in which a Fluent macro called CG_MOTION was utilized. The dynamically moving structured mesh was specified as a rigid body in which the location of the CG for each of the rigid body parts was identical. Figure B-6 provides an illustration of the configurations for the dynamic mesh zones. As the simulation progressed, the X-component of the CG location was automatically updated. For the baseline and taildeployed configurations, the simulation required rotation about the negative $\mathrm{X}$-axis in addition to translation. 


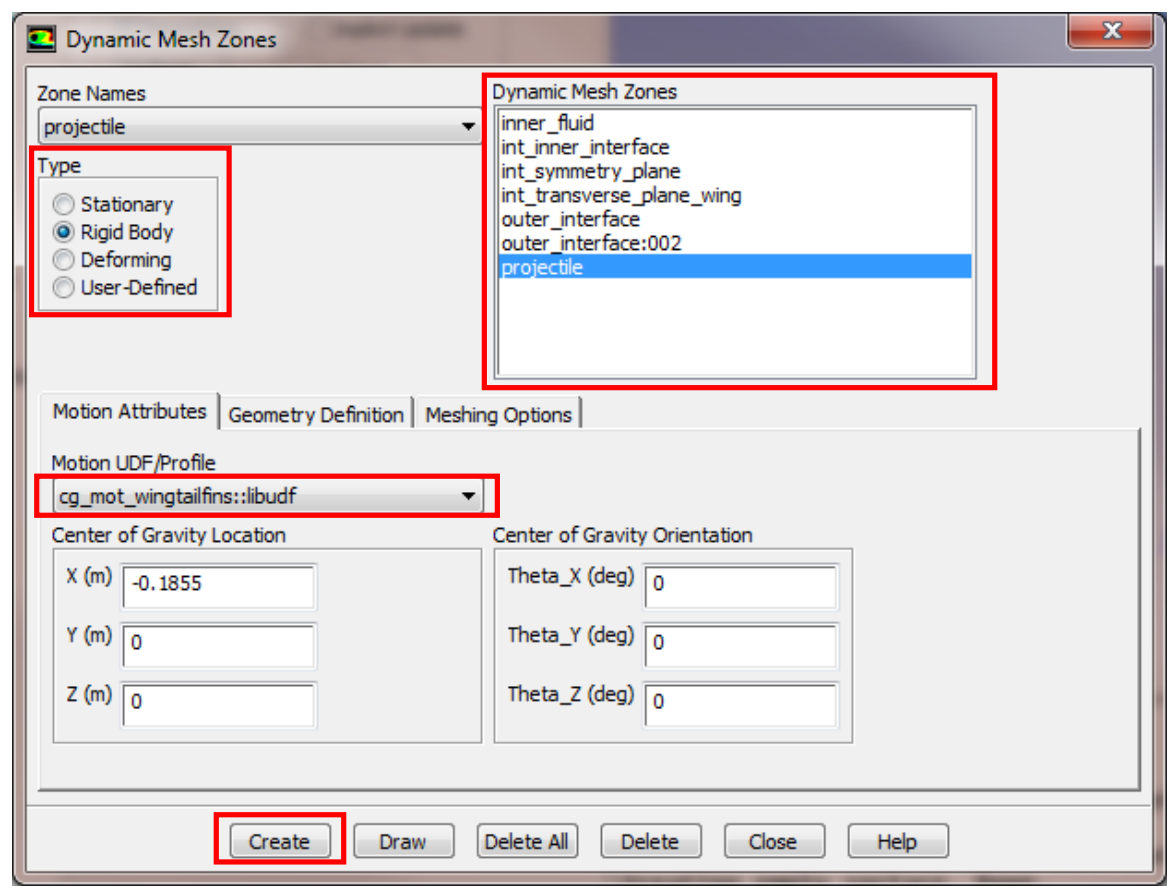

Figure B-6: Dynamic Mesh Zone Configurations for Initial Simulation

After a sufficient number of time steps had been performed, the 6-DOF solver was activated to simulate free body motion. The options for the 6-DOF solver allowed for motion to be written to a designated file. Figure B-7 and Figure B-8 provide the configurations to conduct the free body motion analysis.

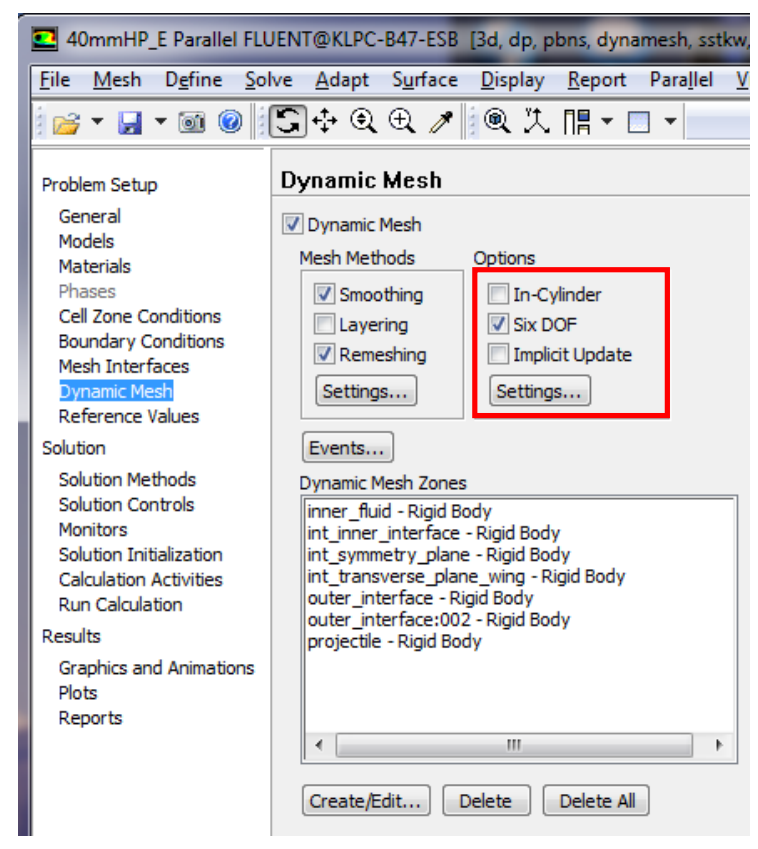

(a)

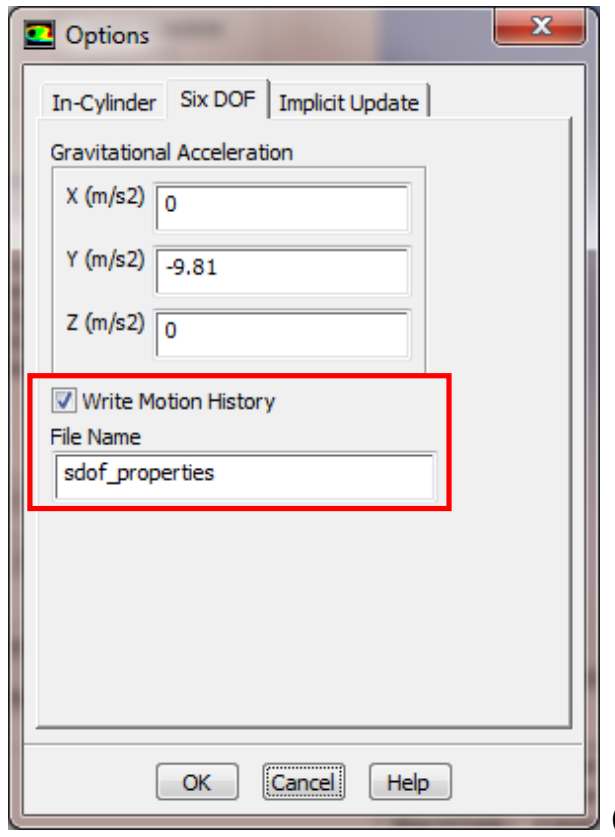

(b)

Figure B-7: Configuration Settings for (a) Dynamic Mesh Setup and (b) 6-DOF Options

The dynamic mesh zone settings were updated for executing the 6-DOF solver, which required a different source code to be selected. This UDF used a Fluent macro called SDOF_PROPERTIES in 
which the mass and moments of inertia were specified. This information was used to determine the twelve parameters shown in the figure below. Also, the Passive option was selected for all the dynamic mesh zones listed except for the projectile. This option was checked to ensure that the aerodynamic forces and moments would not be taken into account for dynamic mesh zones except for the aerodynamic body. As the simulation advanced, the twelve parameters were automatically updated.

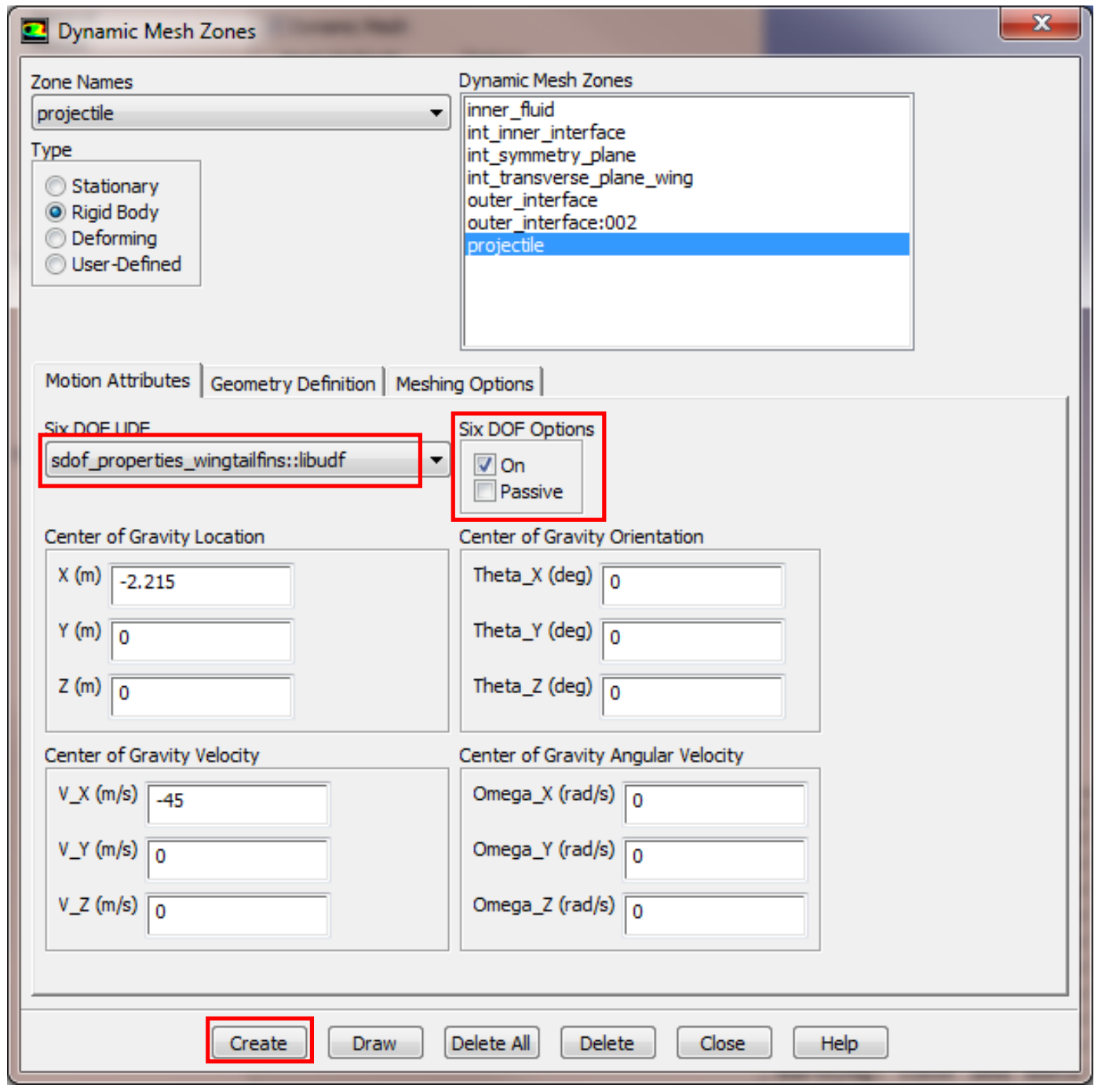

Figure B-8: Dynamic Mesh Zone Configurations Settings for 6-DOF Simulation 


\title{
Appendix C Curriculum Vitae
}

\author{
William L. Vogel
}

(304)615-5992・wvogel86@gmail.com

\section{Personal Profile}

Upcoming graduate of ABET-accredited master's degree program seeking an entry-level position as an aerospace or mechanical engineer. Acquired prior work experience in the aerospace industry with the Boeing Company and Northrop Grumman Corporation. Comprehensive list of engineering design and analysis software experience.

\section{Education}

January 2011 - December 2012

West Virginia University CEMR

Master of Science, Aerospace Engineering Morgantown, West Virginia

- Thesis Title: Numerical Flight Testing of a Tube-launched Transformable Micro Air Vehicle

- Grade Point Average: 3.50

August 2005 - December 2010

West Virginia University CEMR

Bachelor of Science, Mechanical and Aerospace Engineering

Morgantown, West Virginia

- Grade Point Average: 3.34

\section{Relevant Coursework}

Computational Fluid Dynamics - Turbulent Fluid Flow - Flight Mechanics and Controls - Flight Simulation • Guided Missile Systems • Structural Mechanics • Aerospace Structures - Structural Finite Element Analysis • Flight Vehicle Design

\section{Work Experience}

January 2010 - May 2010

Attitude Control Systems Engineering Co-op for NSROC Program

- Developed graphical user interface to read Real Time Attitude Solution flight data through selected COM port

- Supported engineers in running test to collect force and pressure data for nozzles inside vacuum chamber

- Supported flight performance engineers in analyzing accelerometer, vibrometer, and motor pressure data
Northrop Grumman Corporation Wallops Island, Virginia 


\section{Work Experience (Continued)}

May 2008 - August 2008; May 2009 - August 2009

C-17 Field Services Engineering Intern

- Authored deficiency reports, field service reports, and engineering dispositions for failed parts on aircraft

- Examined Standard Flight Data Recorder for failed landing gear

- Solved wiring problems to various aircraft systems

- Performed field testing of failed multi-junction probes

June 2007 - August 2007

Propulsion Engineering Intern

- Supported engineers in development of 747-8 engine control/airplane interface by compiling Boeing 747/777 data
The Boeing Company

Charleston AFB, South Carolina

\section{Honors and Activities}

- Member, American Institute of Aeronautics and Astronautics, August 2006 - Present

- Member, WVU Unmanned Aerial Vehicle Design Team, August 2006 - May 2007

- West Virginia Promise Scholar

- Jerome B. Fanucci Scholar

- Kirkland Scholar

- Eagle Scout, Boy Scouts of America

\section{Relevant Skills}

- Experience with ANSYS Fluent CFD package

- Experience with ANSYS ICEM CFD package

- Experience with ANSYS structural analysis package

- Experience with Tecplot 360 package

- Experience with SolidWorks CAD package

- Experience with FDC flight simulation package

- Experience with MATLAB and Simulink packages

- Experience with basic programming in $\mathrm{C} / \mathrm{C}++$

- Experience with high performance computing machines

- Experience with all standard digital office applications

\section{References}

References are available upon request 